\title{
Atomic Force Microscopy Study of the Interactions Between Colloids and Surfaces - Membranes and Cells
}

He, Yuan

How to cite:

He, Yuan (2018) Atomic Force Microscopy Study of the Interactions Between Colloids and Surfaces - Membranes and Cells. Doctoral thesis, Swansea University.

http://cronfa.swan.ac.uk/Record/cronfa43734

Use policy:

This item is brought to you by Swansea University. Any person downloading material is agreeing to abide by the terms of the repository licence: copies of full text items may be used or reproduced in any format or medium, without prior permission for personal research or study, educational or non-commercial purposes only. The copyright for any work remains with the original author unless otherwise specified. The full-text must not be sold in any format or medium without the formal permission of the copyright holder. Permission for multiple reproductions should be obtained from the original author.

Authors are personally responsible for adhering to copyright and publisher restrictions when uploading content to the repository.

Please link to the metadata record in the Swansea University repository, Cronfa (link given in the citation reference above.)

http://www.swansea.ac.uk/library/researchsupport/ris-support/ 


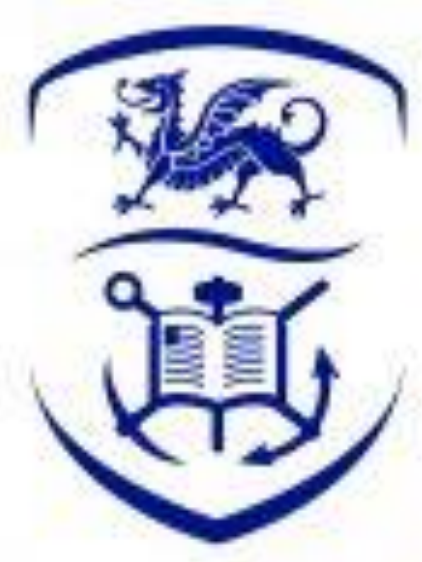

\title{
Swansea University Prifysgol Abertawe
}

\author{
Chemical Engineering, College of Engineering \\ Swansea University
}

Atomic Force Microscopy Study of the Interactions Between Colloids and Surfaces - Membranes and Cells

by

Yuan He

A Thesis submitted of the Requirements for the degree

Doctorate of Philosophy (Ph.D.)

$8^{\text {th }}$ August 2018 


\section{Declaration}

This word has not previously been accepted in substance for any degree and is not being concurrently submitted in candidature for any degree.

Signed (Candidate: Yuan He)

Date

Statement 1

This thesis is the result of my own investigations, except where otherwise stated.

Other sources are acknowledged by footnotes giving explicit references, A bibliography is appended.

Signed (Candidate: Yuan He)

Date

Signed (Supervisor: Dr. Chris Wright)

Date

\section{Statement 2}

I hereby give consent for my thesis, if accepted, to be available for photocopying and for inter-library loan, and for the title and summary to be made available to outside organizations

Signed (Candidate: Yuan He)

Date 


\section{Acknowledgment}

I wish to express my sincere gratitude to Dr. Chris Wright for the kindly supervision of the research project. The whole project is progressing with his kindly suggestion and direction.

I would also say thanks to Dr. Paul Williams for his help on FORTRAN code development and zeta potential study of the project.

I am also thanks Dr. Alex Lord for the help on AFM training and SEM imaging. 
Acronyms

AFM

DLVO

DMT model

JKR model

MWW

MYGP

NCYC

OD

P.F

SFA

SEM

TEM atomic force microscopy

a theory developed and named after Boris Derjaguin, Lev Landau, Evert Verwey and Theodoor Overbeek

model developed by Derjagin, Muller, Toropov in 1975

model developed by Johnson, Kandall, Roberts in 1971

Mann-Whitney U test

universal medium for yeasts

national collection of yeast cultures

optical density

Pseudomonas fluorescens

surface force apparatus

scanning electron microscopy

transmission electron microscopy 


\section{Nomenclature}

A $\quad$ - Hamaker constant

- Surface contact area of colloid probe

a

- Particle radius in double layer model

- Radius of contact area of colloid probe

C - Concentration

D - Cohesive energy term constant

E - Young's modulus $(\mathrm{Pa})$

e - Electric unit charge

F $\quad-$ Force

$F_{w l c} \quad-$ Wormlike chain force

$g \quad$ - Gradient of derived equation

h - Height of each integration step of particle sphere surface

$\mathrm{H} \quad$ - Gap between sample and bottom of bead

J - Thermionic emission electron current density

$\mathrm{k} \quad$ - Boltzmann constant $(\mathrm{J} / \mathrm{K})$

$k_{c} \quad-$ Spring constant of cantilever $(\mathrm{N} / \mathrm{m})$

$k_{B} \quad-$ Boltzmann constant

L $\quad$ - Length of cantilever (m)

$m^{*} \quad-$ Effective mass of the cantilever $(\mathrm{g})$

$m_{p} \quad$ - Persistence length

$n_{i} \quad-$ Ionic concentration on location at a distance from the charged surface

$n_{i}{ }^{o} \quad$ - Ionic concentration on charged surface

OD - Optical density test result in \%

r $\quad$ - Particle radius

s $\quad-$ Surface stress $(\mathrm{N} / \mathrm{m})$

t $\quad-$ Time

$\mathrm{T} \quad$ - Temperature in $\mathrm{K}$ 


\begin{tabular}{|c|c|}
\hline$t_{c}$ & - Thickness of cantilever (m) \\
\hline$v_{o}$ & - Resonance frequency of cantilever \\
\hline$V_{T}$ & - Van der Waals interaction \\
\hline$V_{A}$ & - Non-retarded Van der Waals interaction \\
\hline$V_{R}$ & - Retarded Van der Waals interaction \\
\hline $\mathrm{W}$ & - Wide of cantilever \\
\hline$w_{i}$ & - Free energy stored in electric potential \\
\hline $\mathrm{x}$ & - Percentage of reacted chemical functional groups on cell surface \\
\hline & - Contour length of the molecule \\
\hline$z_{i}$ & - Valency of ion \\
\hline$Z_{c}$ & - Deflection of the cantilever on the end (m) \\
\hline$\Psi$ & - Electric potential at detecting position \\
\hline$\varepsilon_{o}$ & - Vacuum permittivity $(\mathrm{F} / \mathrm{m})$ \\
\hline$\varepsilon_{r}$ & - Relative permittivity \\
\hline$\kappa$ & - Debye length (m) \\
\hline$\Psi_{o}$ & - Surface charge \\
\hline$\Psi_{o}$ & - Zeta potential \\
\hline$\omega_{o}$ & - Angular resonance frequency of the cantilever \\
\hline$\omega$ & - Angular frequency \\
\hline $\mathrm{y}_{D}$ & - Damping coefficient (kg/s) \\
\hline$\delta$ & - Standard deviation \\
\hline & - Surface charge \\
\hline$\Phi$ & - Work function \\
\hline$\rho$ & - Density $\left(\mathrm{kg} / \mathrm{m}^{3}\right)$ \\
\hline
\end{tabular}




\begin{abstract}
Atomic force microscopy (AFM) is an important measurement methodology for the study of interactions at the micro and nanoscale. The study of colloidal interactions at microbial cell or membrane surfaces can be significantly extended by the application of AFM imaging and force measurement capabilities to provide unique insights into the surface properties and their relationships. The zeta-potential of membrane and cell surfaces can be mathematically described with Boris Derjaguin, Lev Landau, Evert Verwey and Theodoor Overbeek (DLVO) theories and linked to surface interactions. The research of this thesis analyses AFM force-distance measurements and has developed a FORTRAN program to calculate surface properties from AFM force spectroscopy. In the first instance the developed AFM measurement platform allowed the determination of zeta-potential at the nanoscale across a membrane surface (DK). The mapping of the zeta-potential across a surface within a process relevant environment alongside the measurement of other surface properties is unique to AFM and the presented thesis.

The distribution of zeta-potential across the membrane or microbial surfaces was found to be as large as $\pm 20 \mathrm{mV}$ with the average zeta potential ranging from $10 \mathrm{mV}$ to a maximum as $35 \mathrm{mV}$ depending on the surface and aqueous environment. The results were compared to other zeta potential measuring methods; zeta-sizer for cells and streaming potential for DK membrane surface. Zeta potential mapping across the surfaces could also be achieved with the AFM method.

The surface adhesion is a prominent feature of force curves and the research of the thesis extended the FORTRAN program for numerical analysis of the force curve. Maximum adhesion could be measured as more than $10000 \mathrm{pN}$, while minimum could be less than $100 \mathrm{pN}$. Hydrophobicity of cells was also measured to aid interpretation of AFM data. With a combination of reaction equilibrium and Gaussian distribution, the research demonstrates that the method can identify the type of functional groups on the sample surface.

To illustrate the application of the developed AFM analysis the influence of chemical additives on the surface interactions was also investigated. The effect of Sodium tripolyphosphate (STP) on zeta potential at bacterial and yeast cell surfaces was studied. The effect of STP was to narrow the distribution of zeta potential from $10-20 \mathrm{mV}$ to $10-15 \mathrm{mV}$ for both yeasts and bacteria. The influence of the antibiotic amoxicillin was also examined and there was a significant adhesion detected with the non-amoxicillin treated cells; maximum of about $3000 \mathrm{pN}$ for NCYC-1324 and maximum of around $30000 \mathrm{pN}$ for NCYC-1681. The adhesion was reduced to a few hundred $\mathrm{pN}$ within $15 \mathrm{mins}$ in low amoxicillin $(0.1 \mathrm{mg} / \mathrm{l})$. A longer time of exposure or higher concentration caused damage to the cell and reduced the validity of the cell adhesion measurement.

In conclusion, the work of the thesis has developed an AFM analysis platform that allows the novel interrogation of AFM force-distance curves measured across surfaces. This provides unique insight into the interactions found at the surface which govern the behaviour of colloids and bio-colloids and impacts within medicine, bioprocess engineering and the natural environment.
\end{abstract}




\section{List of Figures}

Figure.1. 1. Schematic representation of the cell membrane (Rogers, 2007).............2

Figure.1. 2. Schematic structure of yeast cell wall structure (McClanahan, 2009)......3 Figure.1. 3. Schematic differences between Gram-positive and Gram-negative bacteria cell wall structures (SimBac, 2013).

Figure.1. 4. Gouy-Chapman-Grahame-Stern model (A), and Gouy- Chapman Model (B), models described with Helmholtz plane. While, IHP present inner Helmholtz plane and OHP is outer Helmholtz plane. In both models, OHP is the edge of modelled layers.

Figure.1. 5. The Counter-ions and Co-ions concentration change vs distance.............7

Figure.1. 6. Potential change over the diffuse part (A), plot of potential vs $1 / k(B) \ldots 8$

Figure.1. 7. The description of electrical potential with the assumed equipotential in the diffuse part for an example of potential plot with distance. Adept from (Hunter, 1989).

Figure.1. 8. The present of the cooperation effect of repulsive and attractive forces in

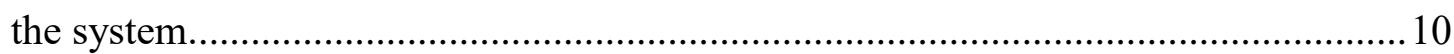

Figure.1. 9. Schematic representation of AFM instrument................................... 14 Figure.1. 10. A diagram showing cooperation between different experiments and analysis.

Figure.2. 1. SEM imaging of the attached silica bead on colloid probe (a) and the provided DNP-10 contact cantilever (b) illustration provided by manufacturer of buker AFM probes.

Figure.2. 2. Figure of name and cooperation of the FORTRAN code programs involved in the computation based numerical analysis model.

Figure.2. 3. The introduction headline of the combined FORTRAN program, introduction of the whole function and their linking in the calculation model. 33

Figure.3. 1. Raw deflection-distance curve imported for calculation (A) and transferred deflection distance-distance curve (B).

Figure.3. 2. Direct calculated force-distance curve from deflection distance-distance curve (A) and finial force-distance for following calculation (B).

Figure.3. 3. AFM force curve with the mean gradient $(G)$ of whole force curve force change assumed in gradient comparison.

Figure.3. 4. Geometry of the silica colloid probe and membrane surface, illustration of the relationship between bead radius (a), cross section radius (r) and local height away from membrane surface $(\mathrm{h})$.

Figure.3. 5. Area of the membrane sample engaged in zeta potential calculation......44 Figure.3. 6. Spherical surface segmented into 6 stages for calculation. Dashed lines show the mean height of the stages.

Figure.3. 7. The composition of total height for each stage to membrane surface. Two parts of the total height include level difference to bottom and bottom to membrane 
distance.

Figure.3. 8. Contact-model scans of (a) DK membrane surface and (b) Cyclopore membrane surface topography with colloid probe of $33.5 \mu \mathrm{m}$. Imaging of membrane surface with contact tip (c) DK membrane and (d) Cyclopore. All images are in size of $9.47 \mu \mathrm{m}$ X $9.47 \mu \mathrm{m}$.

Figure.3. 9. Line analysis of (a) DK membrane and (b) Cyclopore membrane surface, $\mathrm{Y}$ axis is in $\mathrm{nm}$ and $\mathrm{X}$ axis is in $\mu \mathrm{m}$ for both $\mathrm{a}$ and $\mathrm{b}$.

Figure.3. 10. The reality of contact surface. $a$ is the bead surface, $b$ is the real contact surface of membrane, a is the mean flat surface used for FORTRAN calculation. $\mathrm{H}_{\text {mean }}$ is the height used in ideal computation. $\mathrm{H}_{\text {real }}$ is the real distance between two surfaces.

Figure.3. 11. Approximate assumption of the spherical geometry on the membrane surface bending. The parameter of cross-section radius (r) and bending height $(\mathrm{h})$ are measured through AFM imaging.

Figure.3. 12. $a$ is the overall force curve raw data detected by Park XE-100 AFM. $b$ is focus on the contact region on the raw force curve. Point 1,2 and 3 are defined as point contact, 1 point and 2 points before contact. For c, contact point is as 2, point 3 as the point before contact and point 4 as two points before contact. Point 1 is the first point after contact position.

Figure.3. 13. $a$ is the real contact in between the two points involved in zeta potential calculation (contact point and one point before contact); $b$ is the real contact exactly on the contact point. . .54

Figure.3. 14. Errors induced (in percentage) by the differential calculation at different differential steps number used in calculation. .55

Figure.3. 15. Influence of calculation steps on percentage of vertical gap in calculation satisfied the condition of less than $0.1 \%$ probe radius. .56

Figure.3. 16. Plot of van der Waals interaction energy change of flat plates approaching to each other from $10 \kappa$ to contact in $\mathrm{NaCl}$ solution of $0.0001 \mathrm{M}$ (blue), $0.001 \mathrm{M}$ (red) and 0.01M (green).

Figure.3. 17. Double layer considered comparison of electrostatic force and van der Waals forces (a) and its log plot (b) for $40 \mathrm{mV}$ plates in $0.0001 \mathrm{M} \mathrm{NaCl}$ solution, blue for Stoton's simulation from $2 \kappa$ and red for DLVO electrostatics estimation from double layer start overlap.

Figure.3. 18. The influence of probe size (a) in constant $40 \mathrm{mV}$ potential and zeta potential (b) in constant $40 \mu \mathrm{m}$ diameter probe size on ratio between electrostatic force and van der Waals forces.

Figure.3. 19. Treated AFM approaching force curve without a snap in (a) and with a snap in (b). .63

Figure.3. 20. Comparison of the estimation of electrostatic potential at different separation with DLVO theories (red) and Stotons's linearized simulation (blue) (Stoton, 2001).

Figure.3. 21. Zeta potential calculated with the electrostatic potential calculated at different separation distances with Stoton's electrostatic potential simulation (Stoton, 2001) curve with $0.036 \mathrm{~V}$ plates in $0.0001 \mathrm{M} \mathrm{NaCl}$ buffer solution. .65 
Figure.3. 22. a is comparison of calculated zeta potential error with Stoton's simulated curve (blue) and local potential error to DLVO estimation (red) at different separation distance, $\mathrm{b}$ is the exponential comparison of Stoton's simulation (blue) and simulation setting separation (red). Calculation set is $0.0036 \mathrm{~V}$ plates in $0.0001 \mathrm{M}$ $\mathrm{NaCl}$ solution.

Figure.3. 23. Comparison of zeta potential error observed from simulation (a), calculated with error effects on electrostatic potential (b) and influence on separation distance (c). 68

Figure.3. 24. Zeta potential calculated with error reduced simulation curves in $0.0001 \mathrm{M} \mathrm{NaCl}$ solution and $5 \mathrm{mV}$ (blue), $20 \mathrm{mV}$ (red) and $40 \mathrm{mV}$ (green) plates. ....69 Figure.3. 25. Zeta potential calculated with error reduced simulation curves in set zeta potential of $5 \mathrm{mV}$ (a), $20 \mathrm{mV}$ (b) and $40 \mathrm{mV}$ (c) in ionic strength of $0.0001 \mathrm{M}$ (blue), $0.001 \mathrm{M}$ (red), $0.01 \mathrm{M}$ (green) and $0.1 \mathrm{M}$ (purple) $\mathrm{NaCl}$ solutions compare with set zeta potential in simulation (cyan). 70 Figure.3. 26. Error induced by the surface charge density calculation at $5 \mathrm{mV}$ (blue), $20 \mathrm{mV}$ (red) and $40 \mathrm{mV}$ (green) in different ionic strength. Ionic strength was plot in the form of $-\log$ for better illustration.

Figure.3. 27. the normal distribution shifting of $n \delta$ with the equilibrium reaction and buffer concentration controlled surface hydrophilic groups density from the test of $\mathrm{OD}=50 \%$.

Figure.3. 28. example plot of $\mathrm{G}$ vs $\mathrm{K}_{\mathrm{E}}$ of two randomly $\mathrm{n}$ values used. 78

Figure.3. 29. (A) is the possibility of real data recorded of 1000 points random integer value distributed at each value, and (B) is comparison of the random generated data distribution (Orange) and its simulated Gaussian distribution (Blue).... .81

Figure.3. 30. Possibility of real data recorded of 5 groups of 1000 points random integer value distributed at each value (Orange) and its simulated Gaussian distribution (Blue)..... .82

Figure.4. 1. Example of force curves measured on cyclopore membrane surface in different buffer $\mathrm{pH}$ conditions and constant ionic strength $(0.1 \mathrm{M} \mathrm{NaCl}$ solution), a in $\mathrm{pH} 5, \mathrm{~b}$ in $\mathrm{pH} 7$ and $\mathrm{c}$ in $\mathrm{pH} 9$ buffer.

Figure.4. 2. Example of force curves measured on DK membrane surface in different buffer conditions and constant ionic strength $(0.1 \mathrm{M} \mathrm{NaCl}$ solution $)$, a in $\mathrm{pH} 5, \mathrm{~b}$ in $\mathrm{pH} 7$ and $\mathrm{c}$ in $\mathrm{pH} 9$ buffer.

Figure.4. 3. Example of force curves measured on cyclopore membrane surface in different buffer ionic strength and constant $\mathrm{pH}(\mathrm{pH}$ ), a in DI water, $\mathrm{b}$ in $0.001 \mathrm{M}$ $\mathrm{NaCl}$ solution, $\mathrm{c}$ in $0.01 \mathrm{M} \mathrm{NaCl}$ solution and $\mathrm{d}$ in $0.1 \mathrm{M} \mathrm{NaCl}$ solution.

Figure.4. 4. Example of force curves measured on DK membrane surface in different buffer ionic strength and constant $\mathrm{pH}(\mathrm{pH} 5)$, $\mathrm{a}$ in DI water, $\mathrm{b}$ in $0.001 \mathrm{M} \mathrm{NaCl}$ solution, $\mathrm{c}$ in $0.01 \mathrm{M} \mathrm{NaCl}$ solution and $\mathrm{d}$ in $0.1 \mathrm{M} \mathrm{NaCl}$ solution. .92

Figure.4. 5. Zeta potential of silica beads measured with zeta-sizer in different $\mathrm{pH}$ and ionic strengths. Measurements are all in $\mathrm{NaCl}$ solutions or DI water. Purple cross is in DI water, read dot in $0.1 \mathrm{M}$, blue square in $0.01 \mathrm{M}$ and green triangle in $0.001 \mathrm{M}$ of $\mathrm{NaCl}$ solutions. .93 
Figure.4. 6. Average zeta potential of cyclopore membrane surface (a) and DK membrane surface (b). Both plots are with error estimations. Unit is in $\mathrm{mV}$ for both plots

Figure.4. 7. $\mathrm{a}$ is the average zeta potential measured with streaming potential from $\mathrm{pH}$ 3 to 11, cited from (Oatley et al., 2013); b is the streaming potential zeta potential results between $\mathrm{pH}$ of 4 and $10, \mathrm{Y}$ axis as the zeta potential in $\mathrm{mV}$ and $\mathrm{X}$ axis is $\mathrm{pH}$ for both plots, cited from (Thomas et al., 2016).

Figure.4. 8. Zeta potential from tangential streaming potential measurements for the Desal DK nanofiltration membrane with $\mathrm{NaCl}$ as the electrolyte, cited from (Oatley-Radcliffe et al., 2017). .98

Figure.4. 9. The zeta potential distribution on the membrane surface based on $4 \times 4$ matrix AFM force mapping. Cyclopore membrane surface distribution is presented as $\mathrm{a}, \mathrm{b}$ is for the DK membrane surface. Vertical axis (Y) is zeta potential measured in unit of $\mathrm{mV}, \mathrm{X}$ and $\mathrm{Z}$ axis are showing the coordinates of measuring points on the $4 \mathrm{x} 4$ matrix.

Figure.4. 10. 8 × 8 zeta potential mapping of membrane surface; Cyclopre and DK membrane displayed as a and $b$ separately. $\mathrm{Y}$ axis is the zeta potential with unit of $\mathrm{mV}$, $\mathrm{X}$ and $\mathrm{Z}$ axis are showing the coordinates of the matrix

Figure.4. 11. An $8 \times 8$ Matrix zeta potential distribution illustration on DK membrane surface, $\mathrm{Y}$ axis is zeta potential in $\mathrm{mV}, \mathrm{X}$ and $\mathrm{Z}$ axis are the coordinates of matrix.. 103 Figure.4. 12. Percentages of adhesion forces detected on membrane surfaces in different concentration and $\mathrm{pH}$ of buffer solution, used in comparison of $\mathrm{pH}$ effects at different buffer concentrations. Blue is the percentage of adhesion detected on Cyclopore membrane surface and red is for DK membrane surface. 104

Figure.4. 13. Percentage of adhesion detected on both membrane surfaces, compare of buffer concentration effect on surface adhesion for both Cyclopore (blue) and DK (red) membrane surfaces.

Figure.4. 14. Comparison of adhesion percentages of different probe sizes used, blue is for $5 \mu \mathrm{m}$ silica bead colloid probe and red is for $33.5 \mu \mathrm{m}$ silica bead colloid probe. Cyclopore membrane data is present in $\mathrm{a}$ and $\mathrm{b}$ is for DK membrane. 106

Figure.4. 15. Typical adhesions detected on both Cyclopore and DK membrane surfaces. Short term effect adhesion (a) and long-term adhesion (b). 108

Figure.4. 16. Demonstration diagram of repulsive forces on an approaching force curve, with the mathematical illustration of these applied forces, electrostatic force (1), non-linear contact force (2) and approximate linear contact force (1). Where, E is Young's modulus (assumed as constant), $A$ is contact area, $\mathrm{h}$ is penetration distance, $\mathrm{R}$ is colloid radius, $\zeta$ is zeta potential and $\sigma$ is surface charge.

Figure.4. 17. Zoom-in into the region of distance less than $0.05 \mu \mathrm{m}$ and force less than $0.0014 \mathrm{~N} / \mathrm{m}$ for approaching force curves measured with $20 \mu \mathrm{m}$ diameter silica colloid probe on Cyclopore membrane surfaces in buffer of pH5 and $0.1 \mathrm{M}$ (blue dot), $0.01 \mathrm{M}$ (red square), $0.001 \mathrm{M}$ (green diamond) $\mathrm{NaCl}$ solution and $\mathrm{DI}$ water (purple triangle). Start and end points of travelling in double-layer is marked in enlarged markers. 111

Figure.4. 18. Zoom-in into the region of distance less than $0.1 \mu \mathrm{m}$ and force less than 
$0.0015 \mathrm{~N} / \mathrm{m}$ for approaching force curves measured with $20 \mu \mathrm{m}$ diameter silica colloid probe on DK membrane surfaces in buffer of $\mathrm{pH} 5$ and $0.1 \mathrm{M}$ (blue dot), 0.01 $\mathrm{M}$ (red square), $0.001 \mathrm{M}$ (green diamond) $\mathrm{NaCl}$ solution and DI water (purple triangle). Start and end points of travelling in double-layer marked with enlarged markers.

Figure.5. 1. Size distribution plot of NCYC-1324 yeast (a), NCYC-1681 yeast (b) and P.F bacteria (c) at different buffer solution $\mathrm{pH}$ condition. Size distribution is plot at $\mathrm{pH}$ 4 (blue), 5 (red), 7 (green) and 9 (purple) for yeasts (a \& b); only pH5 (blue), 7 (red) and 9 (green) were plotted for P.F bacteria (c).

Figure.5. 2. Size distribution plot of NCYC-1324 yeast (a), NCYC-1681 yeast (b) and P.F bacteria (c) at different buffer solution concentration condition; DI water (purple), $0.001 \mathrm{M}$ (green), $0.01 \mathrm{M}$ (red) and $0.1 \mathrm{M}$ (blue) $\mathrm{NaCl}$ solutions are used in the measurements.

Figure.5. 3. Zeta potential measured with zeta-sizer in different buffer $\mathrm{pH}$ for NCYC-1324 (a), NCYC-1681 (b) and P.F bacteria (c). Y axis is zeta in unit of mV. X axis for is $\mathrm{pH}$. Blue dot represents $0.1 \mathrm{M}$ solution, orange square means $0.01 \mathrm{M}$ buffer, grey triangle is for $0.001 \mathrm{M}$ buffer and yellow diamond is used to identify DI water buffer, which related to about $0.0001 \mathrm{M}$ in ionic strength. 122

Figure.5. 4. Zeta potential measured with zeta-sizer in different buffer concentration (plot with log concentration) for NCYC-1324 (a), NCYC-1681 (b) and P.F bacteria (c). $\mathrm{Y}$ axis is zeta in unit of $\mathrm{mV}$. $\mathrm{X}$ axis for is $\mathrm{pH}$. Blue dot presents $\mathrm{pH} 4$ solution, orange square means $\mathrm{pH} 5$ buffer, grey triangle is for $\mathrm{pH} 7$ buffer and yellow diamond is used to identify pH9 buffer, while P.F bacteria is not tested in pH4 buffers (no blue dots).

Figure.5. 5. The plot is for -zeta potential of NCYC-1324 (a) and NCYC-1681 (b) yeast cell against square root buffer concentration. Curve fitting is applied with an exponential relationship. Blue dot is for $\mathrm{pH} 4$, orange square is for $\mathrm{pH} 5$, grey triangle is for $\mathrm{pH} 7$ and yellow diamond presents $\mathrm{pH} 9$.

Figure.5. 6. The plot is to illustrate the linear relationship between $-1 / \psi$ and buffer concentration for NCYC-1324 (a) and NCYC-1681 (b). Blue dot is for $\mathrm{pH} 4$, orange square is for $\mathrm{pH} 5$, grey triangle is for $\mathrm{pH} 7$ and yellow diamond presents $\mathrm{pH} 9 . \ldots \ldots . . .126$ Figure.5. 7. P.F zeta potential plot of (a) -zeta against sqrt (C) and (b) -1/zeta against $\mathrm{C}$, in $\mathrm{pH} 5$ (blue dot), 7 (orange square) and 9 (grey triangle).

Figure.5. 8. Plot of optical density against pH for NCYC-1324 (a), NCYC-1681 (b) and P.F bacteria (c). Solvent partitioning was in different buffer concentration, blue dot for $0.1 \mathrm{M}$, orange square for $0.01 \mathrm{M}$, grey triangle for $0.001 \mathrm{M}$ and yellow diamond for DI water 128

Figure.5. 9. Plot of optical density against log of buffer concentration for NCYC-1324 (a), NCYC-1681 (b) and P.F bacteria (c). Partitioning experiments were in different buffer $\mathrm{pH}$, blue dot for $\mathrm{pH} 4$ (P.F bacteria not tested in $\mathrm{pH} 4$ ), orange square for $\mathrm{pH} 5$, grey triangle for $\mathrm{pH} 7$ and yellow diamond for $\mathrm{pH} 9$.

Figure.5. 10. $\mathrm{G}$ vs $\mathrm{K}_{\mathrm{E}}$ plot of NCYC-1681 (a) and P.F bacteria (b) in pH 5 buffer solutions $(0.1 \mathrm{M}, 0.01 \mathrm{M}, 0.001 \mathrm{M} \mathrm{NaCl}$ solutions and $\mathrm{DI}$ water $), \mathrm{x}$ axis as $\mathrm{K}_{\mathrm{E}}$ and $\mathrm{G}$ on 
y axis, curves is in comparison buffer concentration of blue (a) \& green (b) $=0.1 \mathrm{M}$ to $0.01 \mathrm{M}$, red $(\mathrm{a} \& \mathrm{~b})=0.01 \mathrm{M}$ to $0.001 \mathrm{M}$ and green $(\mathrm{b}) \&$ green $(\mathrm{a})=0.001 \mathrm{M}$ to DI water.

Figure.5. 11. G vs $\mathrm{K}_{\mathrm{E}}$ plot of P.F bacteria in pH5 buffers. (a,) is for $\mathrm{K}_{\mathrm{E}}$ estimation plot only with the two curves based on lower buffer concentration (without the curve plot based on $\mathrm{n}$ values from cells in $0.1 \mathrm{M}$ and $0.01 \mathrm{M} \mathrm{NaCl}$ solution), and (b) is showing the curves with adjusted $\mathrm{n}$ values from cells in $0.1 \mathrm{M} \mathrm{NaCl}$ solution buffer. $\mathrm{X}$ axis as $\mathrm{K}_{\mathrm{E}}$ and $\mathrm{G}$ on $\mathrm{Y}$ axis, curves is in comparison buffer concentration of blue $=0.001 \mathrm{M}$ to DI water, red $=0.01 \mathrm{M}$ to $0.001 \mathrm{M}$ and green $=0.1 \mathrm{M}$ to $0.01 \mathrm{M}$.

Figure.5. 12. Contact mode imaging of NCYC-1324 (a), NCYC-1681 (b) yeast and P.F bacteria (c) with silica colloid probe. Image is in $50 \mu \mathrm{m}$ scale and scanned in $1 \mathrm{~Hz}$. Image measured with silica bead colloid probe (size of 15.6 $\mu \mathrm{m}$ ). Contact tip (DNP-10) is also applied for surface imaging of NCYC-1324 (d), NCYC-1681 (e) and P.F bacteria (f). 142

Figure.5. 13. Geometric illustration of the error applied in imaging of colloid probe used in contact mode imaging. 143

Figure.5. 14. Example of force curves measured on NCYC-1324 yeast cell in different $\mathrm{pH}$ and constant ionic strength, $\mathrm{a}$ is in $\mathrm{pH} 5, \mathrm{~b}$ is in $\mathrm{pH} 7$ and $\mathrm{c}$ is in $\mathrm{pH} 9$ buffer. 145

Figure.5. 15. Example of force curves measured on NCYC-1681 yeast cell in different $\mathrm{pH}$ and constant ionic strength, $\mathrm{a}$ is in $\mathrm{pH} 5, \mathrm{~b}$ is in $\mathrm{pH} 7$ and $\mathrm{c}$ is in $\mathrm{pH} 9$ buffer. 145

Figure.5. 16. Example of force curves measured on P.F bacteria in different $\mathrm{pH}$ and constant ionic strength, $a$ is in $\mathrm{pH} 5, \mathrm{~b}$ is in $\mathrm{pH} 7$ and $\mathrm{c}$ is in $\mathrm{pH} 9$ buffer.

Figure.5. 17. Zeta potential measured on hydroxyapatite beads in different buffer $\mathrm{pH}$ and ionic strengths with zeta-sizer. Measurements are all in $\mathrm{NaCl}$ solutions or DI water. Purple cross is in DI water, read square is in $0.1 \mathrm{M}$, blue dot is in $0.01 \mathrm{M}$ and green triangle is in $0.001 \mathrm{M}$ of $\mathrm{NaCl}$ solutions.

Figure.5. 18. Example comparison of frequency of calculated zeta potential (blue-dot) and its Gaussian distribution (red-line), real data regarded as with significant error were marked as black-triangle. Measurement based on NCYC-1681 cell in $0.01 \mathrm{M}$ $\mathrm{NaCl}$ pH5 buffer.

Figure.5. 19. Gaussian distribution estimation based on Gaussian distribution, a for NCYC-1324, b for NCYC-1681 and c for P.F bacteria. $\mathrm{pH} 5$ shows in blue, $\mathrm{pH} 7$ is in red and green for $\mathrm{pH} 9$. Unit of zeta potential is in $\mathrm{V}$.

Figure.5. 20. Gaussian distribution estimation based on MWW model, data corrected with zeta-sizer results, a for NCYC-1324, b for NCYC-1681 and c for P.F bacteria. $\mathrm{pH} 5$ shows in blue, $\mathrm{pH} 7$ is in red and green for $\mathrm{pH}$ 9. Unit of zeta potential is in V.151 Figure.5. 21. Typical retraction force curve with adhesion detected; including large adhesion with long influencing distance (a), short influencing distance (b), with fluctuation (c); small adhesion with short influencing distance and clear breakoff (d), fluctuation (e), partial breakaway together with smooth and long-distance change (f), with significant influence of contact $(\mathrm{g})$, smooth breakaway $(\mathrm{h})$, fluctuation together with long-distance release (i). $\mathrm{X}$ axis is distance in meter; $\mathrm{Y}$ axis is force in newton. 
Figure.5. 22. Accumulation of adhesion detected percentage with adhesion type increasing. Blue diamond is for silica colloid probes with NCYC-1324, red square is for silica colloid probes with NCYC-1681, green triangle is for hydroxyapatite colloid probes with NCYC-1324, purple cross is for hydroxyapatite colloid probes with NCYC-1681 and cyan plus is for hydroxyapatite colloid probes with P.F bacteria. . 160 Figure.5. 23. The chemical structure of STP (A) and reaction mechanism of reaction between cell surface protein and STP (B). 162

Figure.5. 24. Gaussian distribution estimation for $\mathrm{STP}$ added buffer $(0.01 \mathrm{M} \mathrm{NaCl}$ and 1g/L STP) measurement, a for NCYC-1324, b for NCYC-1681 and c for P.F bacteria. $\mathrm{pH} 5$ shows in blue, $\mathrm{pH} 7$ is in red and green for $\mathrm{pH}$. Unit of zeta potential is in $\mathrm{mV}$.

Figure.5. 25. Typical force curve measured on NCYC-1324 yeast cell in $0.4 \mathrm{ml} / \mathrm{L}$ amoxicillin buffer with exposure time of $15-30$ minutes. 168

Figure.5. 26. Contact mode imaging of NCYC-1324 yeast cell samples in $0.4 \mathrm{mg} / \mathrm{L}$ amoxicillin buffer (a) exposed for 15 - 30 minutes and (b) 60 - 75 minutes. Both images are $50 \mu \mathrm{m}$ square. 168

Figure.5. 27. Contact mode imaging of NCYC-1324 yeast cell sample in $1 \mathrm{mg} / \mathrm{L}$ amoxicillin buffer (a) exposed for 15 - 30 minutes and (b) 60 - 75 minutes. Both images are $50 \mu \mathrm{m}$ square.

Figure.5. 28. Contact mode imaging of NCYC-1324 yeast cell samples in $4 \mathrm{mg} / \mathrm{L}$ amoxicillin buffer (a) exposed for 15 - 30 minutes and (b) 60 - 75 minutes. Both images are $50 \mu \mathrm{m}$ square.

Figure.5. 29. Contact mode imaging of NCYC-1681 yeast cell sample in $0.4 \mathrm{mg} / \mathrm{L}$ amoxicillin buffer (a) exposed for 15 - 30 minutes and (b) 60 - 75 minutes. Both images are $50 \mu \mathrm{m}$ square.

Figure.5. 30. Typical AFM contact mode imaging of P.F bacteria sample surfaces measured in amoxicillin buffers, imaging in scale of $50 \mu \mathrm{m}$ and objects that measured in cell shape were point out with arrows.

Figure.5. 31. The Gaussian distribution analysis plot of amoxicillin of $0.4 \mathrm{mg} / \mathrm{L}(\mathrm{a})$, $1 \mathrm{mg} / \mathrm{L}(\mathrm{b})$ and $4 \mathrm{mg} / \mathrm{L}(\mathrm{c})$ with the difference of exposure time 15 - 30 minutes (red) and $60-75$ minutes (blue) 174 


\section{List of Tables}

Table.1. 1. Review of cell interactions measurements without different techniques by Aggarwal et al. in 2010, list of cells tested, techniques used, parameters measured and first published paper, adept to (Aggarwal et al., 2010).

Table.1. 2. Representative researches based on colloid probe and AFM force measurement from 2004 to 2015, type of both contact surfaces, force measured and authorizer are listed in table.

Table.2. 1. Ingredient of MYGP agar, formula in the concentration of $g / 1$ in water-based solution.

Table.5. 1. The $\mathrm{n}$ value estimated with OD density test with Buffer / Hexadecane liquid partitioning OD remaining from Spectrophotometer test for NCYC-1324, NCYC-1681 and P.F bacteria. HS is saturated to the hydrophilic side of reaction equilibrium. P.F bacteria is not tested in $\mathrm{pH} 4$, therefore all marked as NA at $\mathrm{pH} 4 . . .131$ Table.5. 2. Comparison of calculated $n$ value and $K_{E}$ adjusted $n$ value for NCYC-1681. 136

Table.5. 3. $\mathrm{K}_{\mathrm{E}}, \mathrm{x}_{50 \%}, \delta_{\%}$ and $\mathrm{x}$ value for NCYC-1681 in pH4 \& 5 buffers and P.F bacteria in pH5, $7 \& 9$ buffers. $0.1 \mathrm{M}, 0.01 \mathrm{M}, 0.001 \mathrm{M} \mathrm{NaCl}$ solution and DI water were used as buffer.

Table.5. 4. Minimum, maximum, average adhesion force strength and number of force curves detected with adhesion force found in NCYC-1324, NCYC-1681 yeast cell - silica bead contact and P.F bacteria - hydroxyapatite bead contact. 154 Table.5. 5. Minimum, maximum, average adhesion force strength and number of force curves detected with adhesion force found in NCYC-1324, NCYC-1681 cerevisiae cell - silica colloid probes interactions and P.F bacteria - hydroxyapatite colloid probes interactions in $1 \mathrm{~g} / \mathrm{L} \mathrm{STP}$ molecule added $0.01 \mathrm{M} \mathrm{NaCl}$ buffers at different $\mathrm{pH}$. 164 


\section{Context Table}

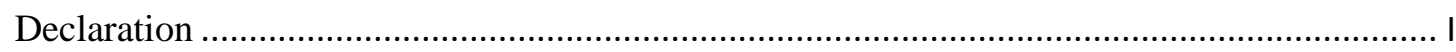

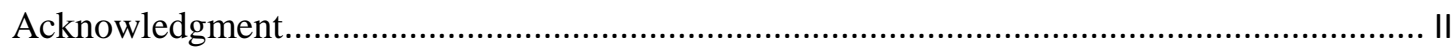

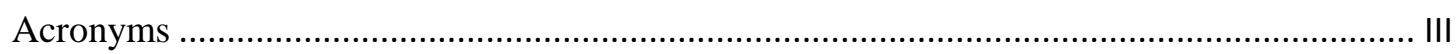

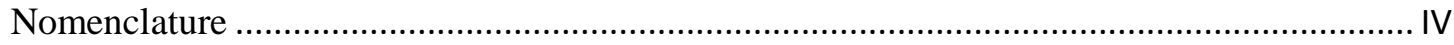

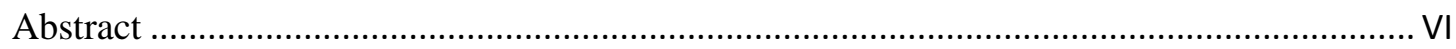

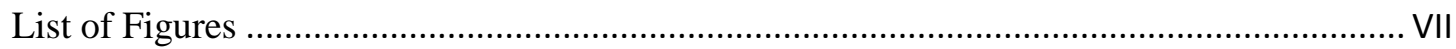

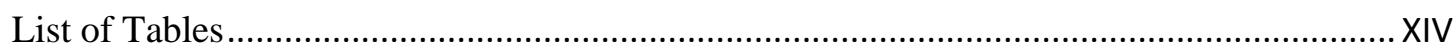

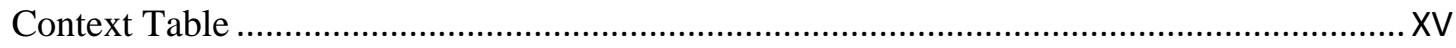

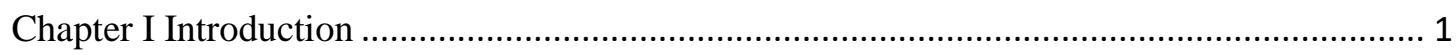

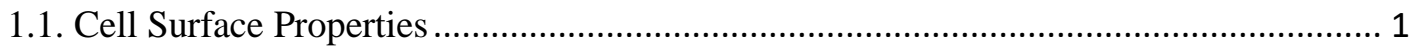

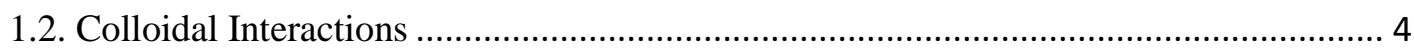

1.2.1. The Theoretical Framework of Colloidal Interactions ………………................... 5

1.2.2. Membrane Filtration - a Process Controlled by Colloidal Interactions ................ 11

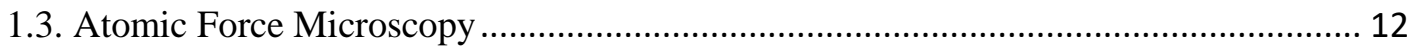

1.3.1. Principles of Atomic Force Microscopy ............................................................... 13

1.3.2. AFM Measurement of Colloidal Interactions ...................................................... 15

1.4. AFM Colloidal Interaction Study - the Research Gap ............................................ 18

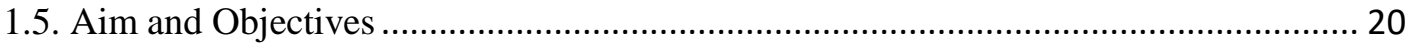

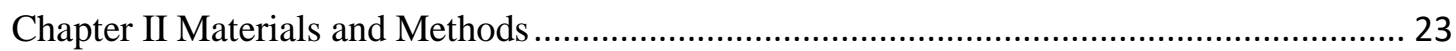

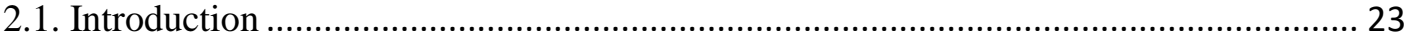

2.2. Experiments Protocols and Preparation.................................................................... 23

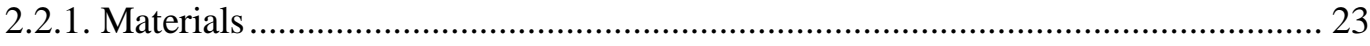

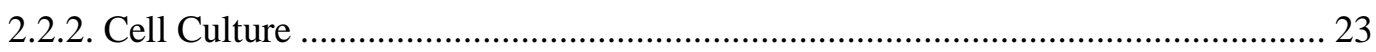

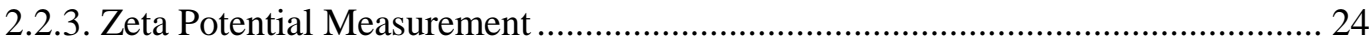

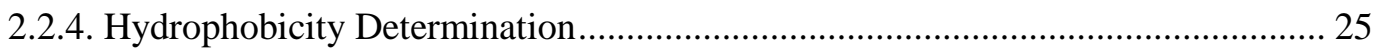

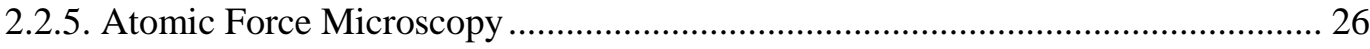

2.2.5.1. Atomic Force Microscopy Sample Preparation.............................................26

2.2.5.1.1. Membrane Sample Preparation .....................................................26

2.2.5.1.2. Cell Sample Preparation .........................................................27

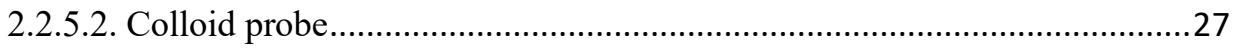


2.2.5.4. AFM Force Measurement and Mapping ...............................................29

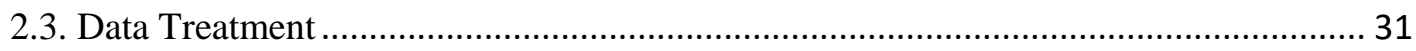

2.3.1. FORTRAN Programming and Analysis of Force Curve.................................. 31

2.3.2. Statistical Analysis ............................................................................ 33

Chapter III Model Development .......................................................................... 34

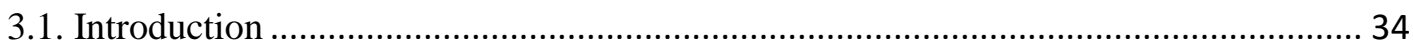

3.2. Force Curve Analysis Model with FORTRAN Code and Calibration ....................... 35

3.2.1. Import and Read of Deflection-Distance Curve ............................................ 35

3.2.2. Transformation of Deflection-Distance Curve to Force-Distance Curve ............. 35

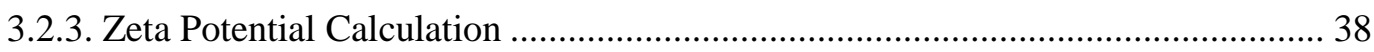

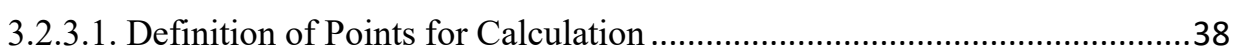

3.2.3.2. Energy Calculation from AFM Force Curves ........................................40

3.2.3.3. Geometric of Spherical Surface Contact with Flat Surface........................42

3.2.3.4. Integration across the Spherical Surface Geometry .................................43

3.2.3.5. The influence of Membrane Surface Deformation on the Zeta Potential Measurement from AFM Force Curve ..............................................................46

3.2.4. Adhesion Analysis from Force Curves ..................................................... 50

3.2.4.1. Maximum Adhesion Analysis ...................................................... 50

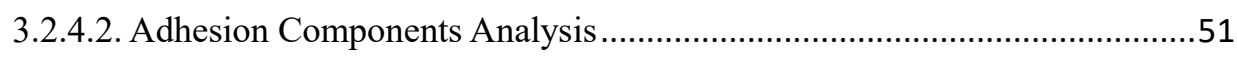

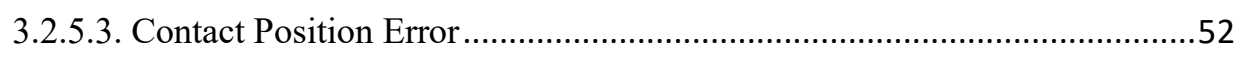

3.2.6. Validation and Calibration of Force Calculation in the FORTRAN Code............ 54

3.2.6.1. Calibration of Surface Differential Calculation for Precision Control .......54

3.2.6.2. Assessment of Impact of the Influence of Van der Waals Force in the

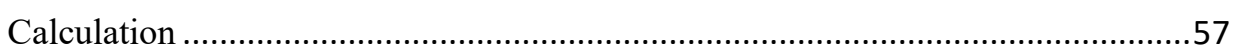

3.2.6.3. Validation and Calibration of Zeta Potential Calculation..........................63

3.3. Development of Hydrophobicity Analysis Model................................................ 72

3.3.1. Solvent Drag Force Analysis of Cells Partitioned in the Different Phases .......... 72

3.3.2. Cell Surface Hydrophobicity Analysis of Cell with Reaction Equilibriums ........ 74

3.3.3. Numerical Analysis of Relationship between Aqueous Phase Optical Density and

Cell Surface Hydrophobicity

3.3.4. Numerical Conversion of Gaussian Distribution to Determine the Cell Surface

Hydrophilic Density in the Reaction Equilibrium ........................................... 77

3.4. Statistical Analysis Model and Data Display ...................................................... 79

3.4.1. Statistical Analysis and Data Display Based on Gaussian Distribution ............... 80 


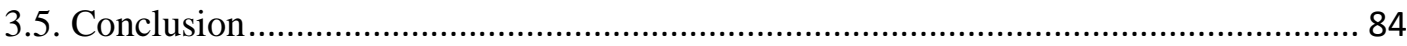

Chapter IV AFM Force Analysis of Particle Interaction with Membrane Surface ................. 86

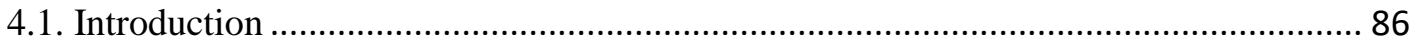

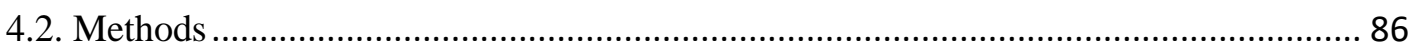

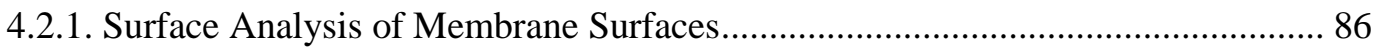

4.2.2. AFM Measurements of Membrane Surfaces ......................................................... 86

4.2.3. Application of Computational Analysis System for Membrane Surface Force

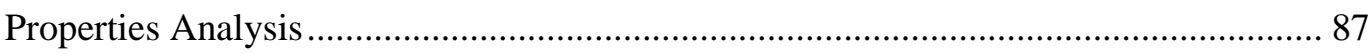

4.3. Membrane Surface Zeta Potential Calculation Using Atomic Force Spectroscopy ..... 87

4.3.1. AFM Force Curves Measured in Aqueous Environment ...................................... 88

4.3.2. Zeta Potential Calculation on Membrane Surfaces with AFM Force Curves ....... 93

4.3.3. Review of Membrane Surface Zeta Potential with Previous Research Based on

Streaming potential and Comparison with AFM Zeta Potential Estimation ................... 95

4.4. Zeta Potential Surface Distribution across a Membrane and the Influence of the

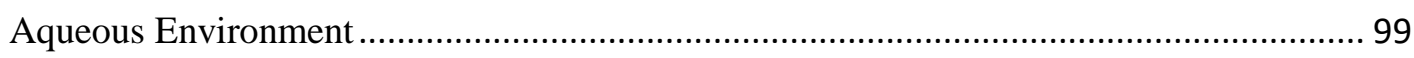

4.4.1. Zeta Potential Distribution Analysis with AFM Force Mapping .......................... 99

4.4.2. Influence of the Aqueous Environment on Zeta Potential Mapping ................... 102

4.4.3. Improvement of Zeta Potential Mapping............................................................... 103

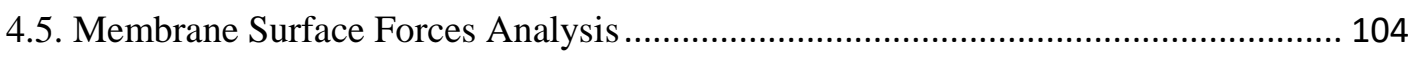

4.5.1. Adhesion Observed on Membrane Surfaces ..................................................... 104

4.5.2. DLVO Forces, Mechanic Forces and Measurement Reliability Analysis........... 109

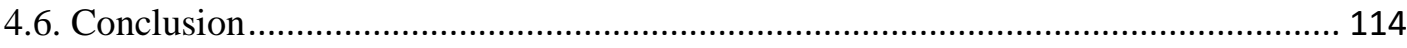

Chapter V Analysis of Intermolecular Forces of Colloid Probe Contact with Cell Surface . 116

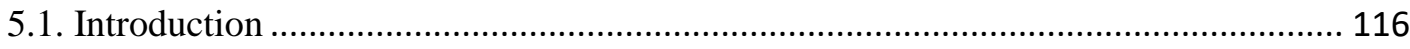

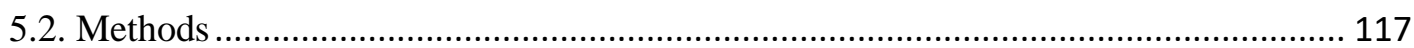

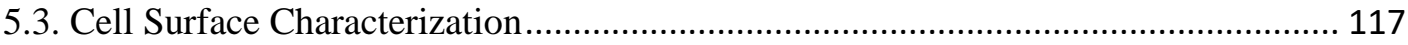

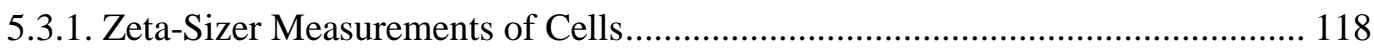

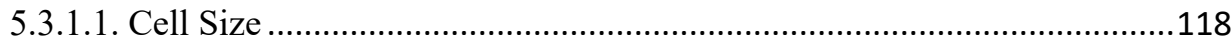

5.3.1.2. Average Cell Zeta Potential Measurement and Analysis..........................121

5.3.2. Measurement and Analysis of Cell Hydrophobicity ........................................ 127

5.3.2.1. The Influence of the Aqueous Environment on Cells in Optical Density 127 
5.3.2.2. Numerical Analysis of Relationship between Aqueous Phase Optical Density and Cell Surface Hydrophobicity........................................................131

5.3.2.2.1. Application of Developed Model in Hydrophobicity Analysis .....131

5.3.2.2.2. Determination and Minimization of Error in Gaussian Distribution Data for Model Values Calculation

5.3.2.2.3. Calculation of the Chemical Group Distribution Which Influence Hydrophobicity

5.3.2.2.4. Analysis of Relationship of the Cell Surface Hydrophobicity and Cell Surface Interactions .

5.4. Imaging and Force Measurement of Cells Immobilized Membrane Surface. 140

5.4.1. Comparison of AFM Contact Mode Imaging with Colloid Probes and Contact Mode Cantilevers.

5.4.2. Force Curves Measured on Cell Surfaces.

5.5. Measurement of Cell Zeta Potential with AFM Force Spectroscopy using Silica and Hydroxyapatite Colloid probes................................................................................. 147

5.6. AFM Adhesion Analysis of Cell Surfaces .............................................................. 153

5.6.1. Maximum Adhesion and Adhesion Distance Analysis ...................................... 153

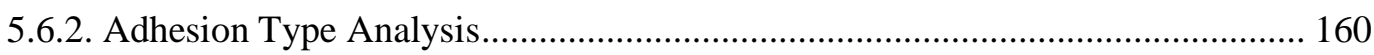

5.7. The Influence of Cell Influential Molecules on Cell Surface Properties.................... 162

5.7.1. The Influence of an Adhesive Controlling Agent on Cell Surface Properties..... 162

5.7.1.1. Influence of Adhesive Controlling Agent on Cell Adhesion ......................163

5.7.1.2. Influence of Adhesive Controlling Agent on Cell Surface Electrostatics. 165

5.7.2. Effects of Antibiotics Used on Yeast Cells Adhesion .......................................... 166

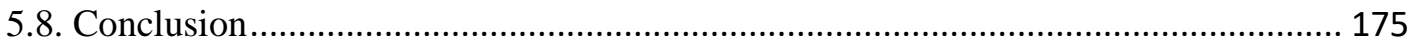

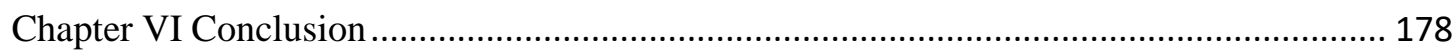

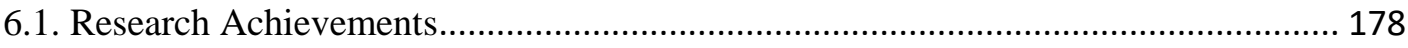

6.2. Application of Research Achievements and Future Research.................................. 181

Reference

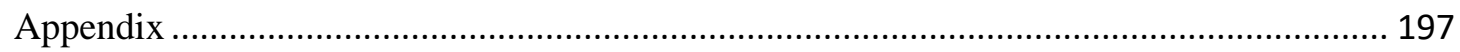

Appendix.1. Hexadecane and Cell Aggregation Error Plot of Optical Density ................ 197

Appendix.1.1. Hexadecane Effects on Spectrophotometer Measurement..................... 197

Appendix.1.2. NCYC-1324 \&1681 Cell Aggregation Effects on Spectrophotometer

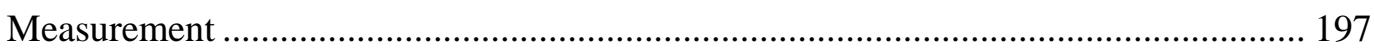

Appendix.1.3. P. fluorescens Cell Aggregation Effects on Spectrophotometer

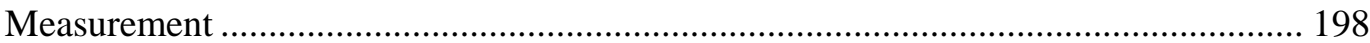


Appendix.2. FORTRAN Code of Data Processing 199 


\section{Chapter I Introduction}

The interactions of particles and surfaces is a fundamental process in natural and industrial systems including medicine and membrane separation. The force of interaction can be a combination of several kinds of intermolecular interactions that include electrostatic force, Van der Waals forces and more specific forces such as ligand-receptor binding.

Electrostatic interaction were found to be effective in the control of membrane filtration (Bellona \& Drewes, 2005).

Drug delivery systems have also be shown to be influenced by intermolecular forces that control the transfer of the drug molecule from carrier particles to target cells (Sosnik et al., 2009).

The study of surface interactions of different particles has been continuing for decades, and the Derjaguin, Lev Landau, Verwey and Overbeek (DLVO) theory is frequently used in these studies. Many kinds of measuring methods have been developed that enable these studies and atomic force microscopy (AFM) has emerged as a prominent device in surface studies as it not only allows nanoscale imaging of surfaces but all the measurement and mapping of interaction forces across surfaces. Cell and membrane surface properties, the DLVO theory and its related developments are reviewed in this chapter. The chapter then considers the application of AFM in colloidal interaction studies in order to identify the research gap and inform the aims and objectives of the thesis.

\subsection{Cell Surface Properties}

The cell surface is an important contact surface in biological systems, its surface properties will directly influence the interactions and subsequent cell behaviour. The cell consists of components such as cytoskeleton, endoplasmic reticulum and plasma 
membrane. The cells metabolism and other activities are accomplished with the cooperation of these different cellular structures. Most of the cells components are not exposed to surroundings and only the cell membrane and cell wall are constantly exposed to outer environments, with these two cell structures providing protection and transfer of materials. The cell membrane is selectively permeable and control the movement of ions, organic molecules or other substances in and out of cells. It consists of a lipid bilayer which is built up by two layers of phospholipids, which have a hydrophilic head and a hydrophobic tail. The hydrophobic tails are facing toward each other in the bilayer, and are not in contact with the outer environments. Many kinds of proteins and glycan are embedded in the membrane, these may be filaments of the cytoskeleton, some penetrate through the membrane and some are found on the surface of the bilayer, as shown in Figure.1.1. The cell membrane is a focal point for the study of cell behaviour as influenced by phenomena, such as cell adhesion and cell signalling; it serves as the attachment surface for extracellular structures and colloid particles (Leroueil et al., 2008).

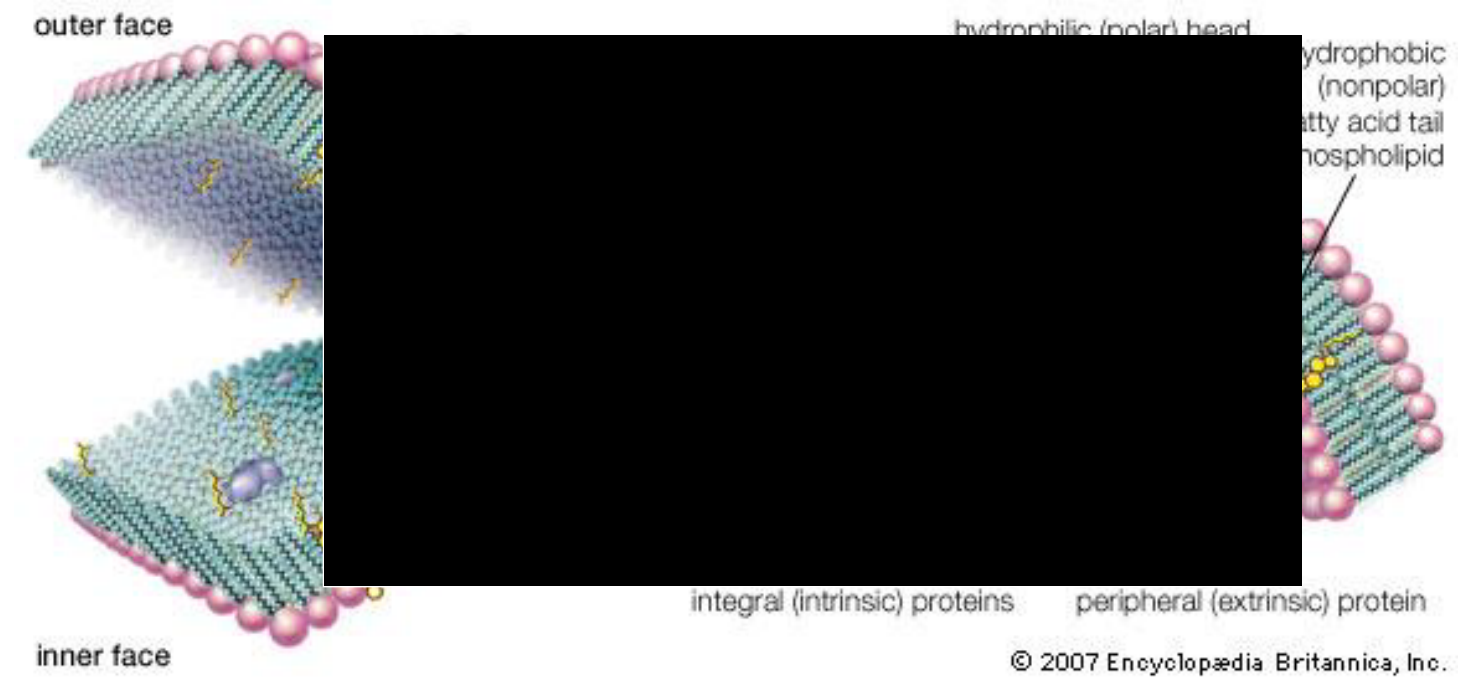

Figure.1. 1. Schematic representation of the cell membrane (Rogers, 2007).

The cell boundary is different depending on the cell type. All cells have a cytoplasmic membrane but this can be accompanied on its outer side by a cell wall. Normally, mammalian cells are not protected by a cell wall. Plant cells, yeast cells and microbial cells are surrounded by cell walls with different chemical structures. 
This study focuses on the interactions of the model organisms yeast (Saccharomyces cerevisiae) and gram-negative bacteria (Pseudomonas fluorescens), thus the structure of these systems are now discussed.

\section{Yeast Cell Wall}

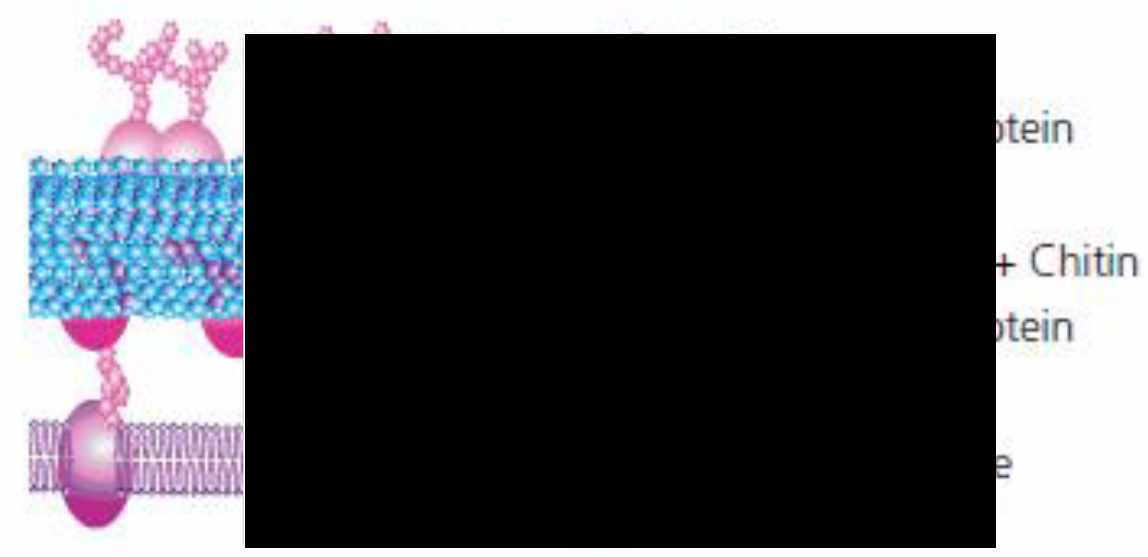

Figure.1. 2. Schematic structure of yeast cell wall structure (McClanahan, 2009).

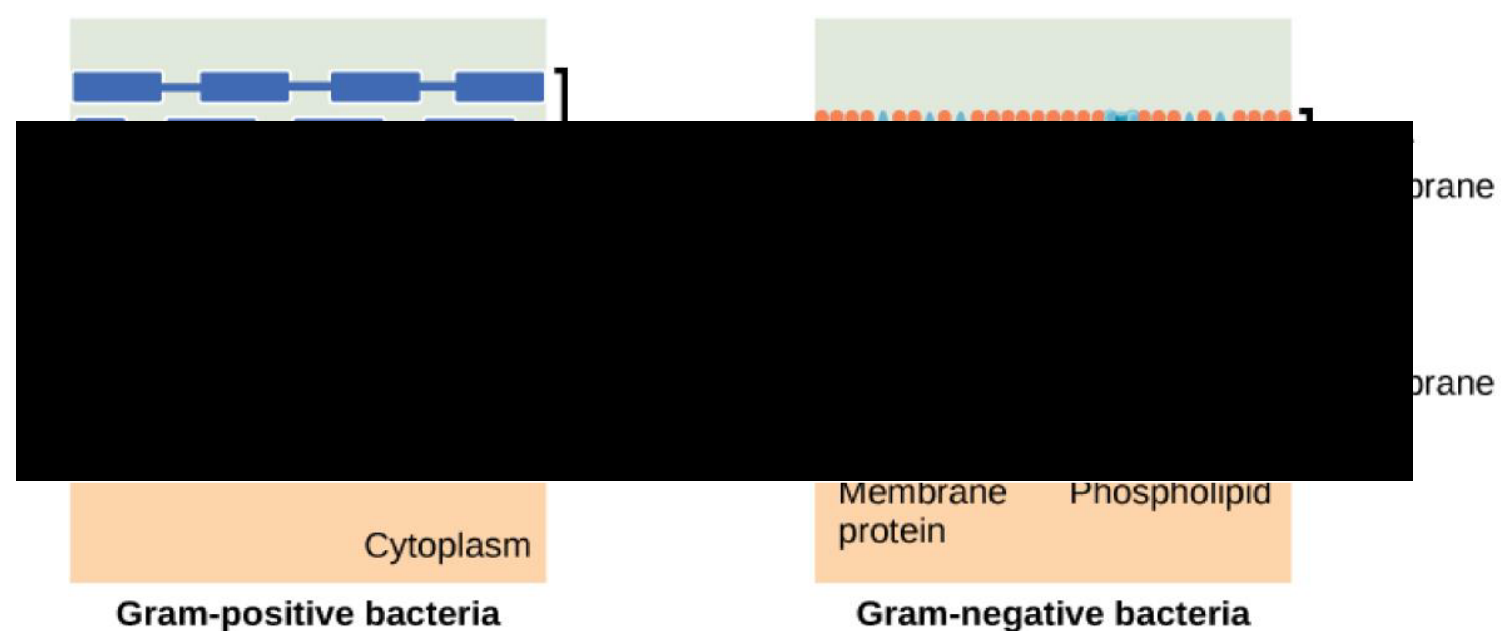

Figure.1. 3. Schematic differences between Gram-positive and Gram-negative bacteria cell wall structures (SimBac, 2013).

The S.cerevisiae cell wall structure was reviewed by Lipke and Ovalle in 1998 (Lipke \& Ovalle, 1998). The major components of the yeast cell wall are large molecules of $\beta 1,3$ glucan and mannoprotein with molecular weights of more than $100 \mathrm{kDa}$ and the relatively small molecules (more than $20 \mathrm{kDa}$ ) of $\beta 1,6$ glucan and chitin. These four components are cross-linked to each other and the cell membrane to 
build the cell wall and contribute to its mechanical strength (Cabib et al., 2001).

Bacteria have a significantly different cell wall compared to yeast cells. There are two types of bacterial cell wall that are differentiated by the bacterial cells ability to process the Gram stain a consequence of the different cell wall structures (Figure.1.2). The Gram-positive cell wall is dominated by a relatively thick layer of peptide glycan compared to a thin layer of the Gram-negative cell wall, which also has an outer membrane-like structure containing lipopolysaccharides. The peptidoglycan layer and its cross-linking provided mechanical strength to the bacterial cell wall (Turner et al., 2013).

The structure of the Gram-negative cell wall was reviewed early in 1974 by Costerton and his colleagues (Costerton et al., 1974). The Gram-negative bacteria are protected by a cytoplasmic membrane, peptidoglycan-lipoprotein complex, periplasmic zone, outer membrane layer and external layer. Some bacteria have protein structures that append the cell wall such as flagella, which have a role in cellular motility and surface attachment, fimbriae and pili can also be present contributing to cell adhesion. Pseudomonas fluorescens (PF) bacteria have flagella on their surface that will contribute to their colloidal behaviour and interaction with surfaces.

\subsection{Colloidal Interactions}

Many systems can be regarded for research and control purposes in terms of the colloidal state. Colloidal systems are an important state of matter which can be defined as small particles of one substance distributed throughout another. For the system to behave as colloidal the distributed particles should be in the size range typically between $1 \mathrm{~nm}$ to a few $1000 \mathrm{~nm}$. The particle size in colloid system is much larger than the molecule size in solutions. Colloid particles are important components of food, drug and beverage products. Below is a review of relevant aspects of colloid science and its application within membrane filtration technologies and the interaction 
of cells.

\subsubsection{The Theoretical Framework of Colloidal Interactions}

Colloid particles are normally the effective particles suspended in the colloidal system. Macro level characteristics of a system can be related to the colloid particles suspend in the system. The interactions are more controllable and easier to experimentally measure if the particles exist in colloid system stably. The interactions applied in the colloid system could be a combination of intermolecular forces including van der Waals forces and electrostatic force. These forces prohibit it turn into unstable and becoming suspension or turbid. For example, electrostatic force are significantly influencing the stability of colloid system, as zeta potential of larger than 25.6mV will induce unstable of colloid system (Verwey \& Overbeek, 1948).

Electrostatic force is of interest to most models that apply colloid science. Several concepts, such as the sterns layer and zeta potential, were created and applied in these models. These models provide a better description of the electrostatics influencing the behaviour of colloid particles, cells or membrane surfaces in a colloidal system.

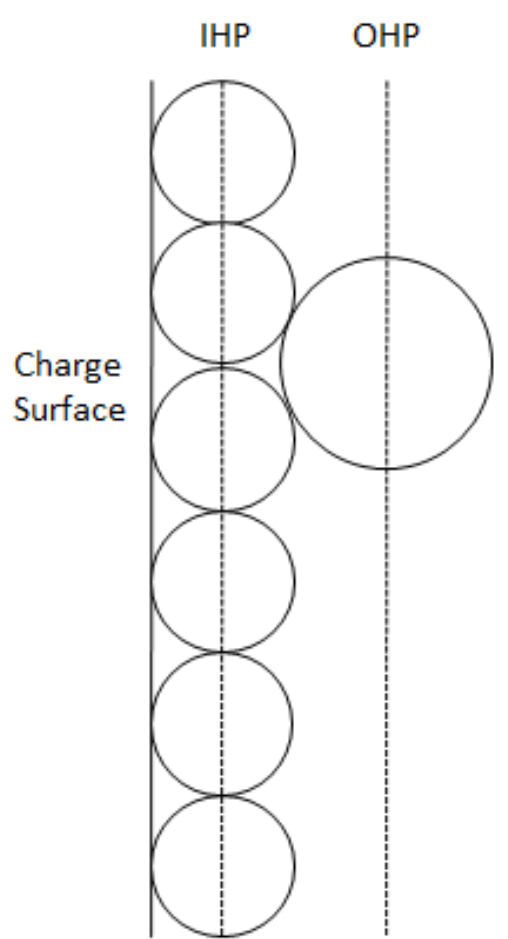

A
Buffer

Solution

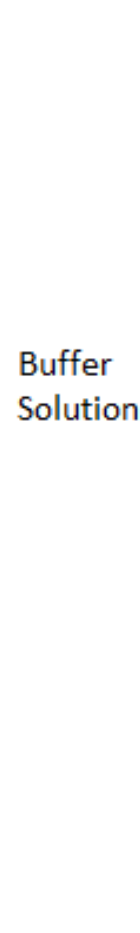

Charge Surface

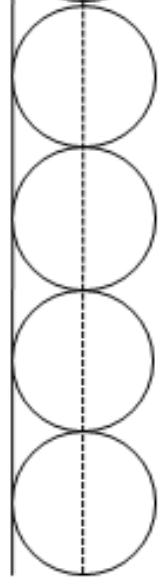

B
Buffer

Solution

Figure.1. 4. Gouy-Chapman-Grahame-Stern model (A), and Gouy- Chapman Model 
(B), models described with Helmholtz plane. While, IHP present inner Helmholtz plane and OHP is outer Helmholtz plane. In both models, OHP is the edge of modelled layers.

Double Layer model accounts for two layers of diffusion of ions attracted to the charge of the surface within an aqueous environment. The double layer model was developed to Gouy-Chapman-Grahame-Stern model (also known as Grahame model) (Figure.1.3 A), which divide the model into 3 regions of IHP, OHP and diffusion (bulk solution). The model was first introduced by Helmholtz and then developed to GouyChapman model (Figure.1.3 B). Stern's layer was then introduced and apply in double layer model, Grahame model was developed with Stern's layer as IHP and a new introduced OHP. In Gouy- Chapman model, the diffuse part (outside OHP of Figure.1.3 B and IHP of Figure.1.3 A) is considered as following the Boltzmann distribution. Ions inside OHP are considered as not diffusing in both models. Solvent are assumed as not influenced by the diffusion throughout the diffuse part, single symmetric electrolyte is assumed with a charge number $\mathrm{z}$ in the system (Verwey \& Overbeek, 1948). So, the use of double layer model is limited, as these assumptions are not always satisfied in real colloid systems.

The zeta potential in double layer theory refers to the electrostatic potential at the OHP, as shown in Figure.1.3 A. Therefore, zeta potential is the electrostatic potential of the charged surface that influences the interactions of the particle with its surroundings and the colloid system. In the bulk solution, the Boltzmann distribution can also be used to describe the electrostatic potential declining (from the zeta potential) with increasing distance from the OHP.

The Boltzmann equation is applied in the analysis of the diffuse part of the double layer model to predict the decline of electrostatic potential and decrease in ion concentration with distance to the charged surface (Figure.1.4). The Boltzmann equation, equation.1.1, can be applied on the mathematic derivation in this model. Where $n_{i}$ is the number of ions at the calculating location, $n_{i}{ }^{0}$ is the number of ions on charged surface, $\mathrm{w}_{\mathrm{i}}$ is the free energy, $\mathrm{k}$ is Boltzmann constant and $\mathrm{T}$ is temperature in 
Kelvin.

$$
n_{i}=n_{i}^{0} \exp \left(\frac{-w_{i}}{k T}\right)
$$

Equation.1.1

Combining equation.1.1 with the Poisson Equation, equation.1.2, a new equation called Poisson- Boltzmann Equation (PBE) is formulated as a derivation equation for spherical shape charged surfaces, equation.1.3 (Verwey \& Overbeek, 1948). The change of zeta potential described in equation.1.3 directly presents as affected by the stress of zeta potential itself. PBE is widely used in calculation of zeta potential in DLVO theories and membrane studies.

$$
\begin{aligned}
& \frac{d^{2} \Psi}{d x^{2}}=\frac{-\rho}{\varepsilon_{0} \varepsilon_{r}} \\
& \frac{d^{2} \Psi}{d x^{2}}=\frac{-1}{\varepsilon_{0} \varepsilon_{r}} \sum_{i} n_{i}^{0} \operatorname{ez} i \exp \left(\frac{-z_{i} e \Psi}{k T}\right)
\end{aligned}
$$

Equation.1.2

Equation.1.3

Where $\Psi$ is electric potential, $\rho$ is charged density, $\mathrm{x}$ is distance, $\varepsilon_{0}$ is permittivity of free space, $\varepsilon_{\mathrm{r}}$ is relative permittivity, $\mathrm{z}_{\mathrm{i}}$ is charge number on each ion, e is the elementary charge.

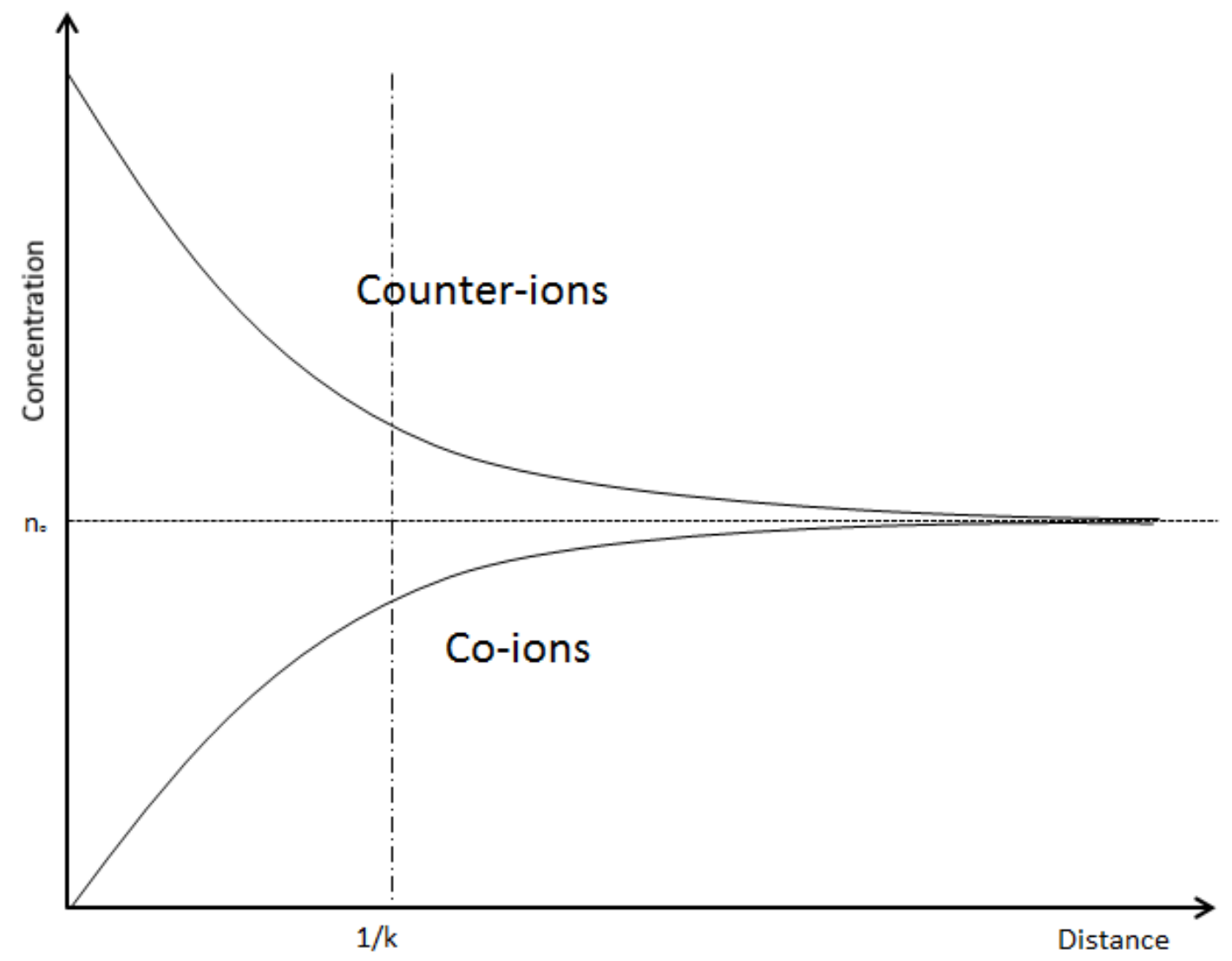

Figure.1. 5. The Counter-ions and Co-ions concentration change vs distance. 
A

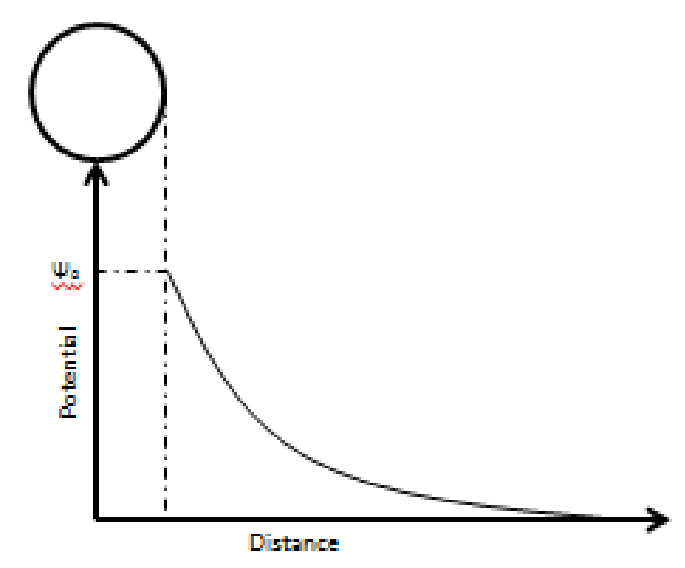

B

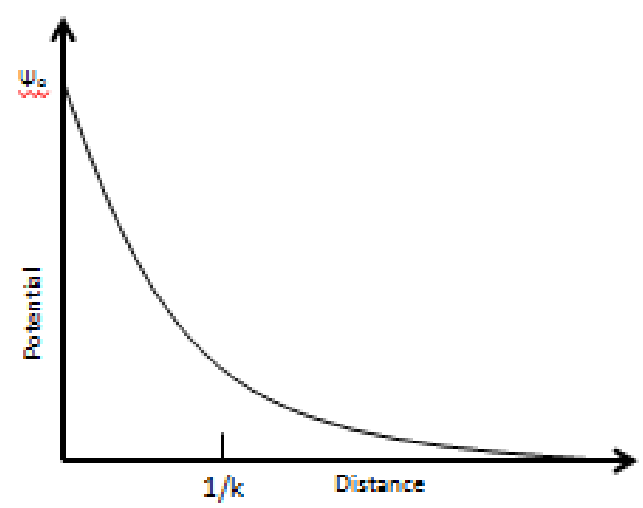

Figure.1. 6. Potential change over the diffuse part (A), plot of potential vs 1/k (B).

Figure.1.5 A shows that the decrease in potential over distance from the flat surface is non-linearly. It is also clear that the zeta potential, at position $1 / \kappa$ (Debye Length), is significantly smaller than the surface potential, Figure.1.5 B.

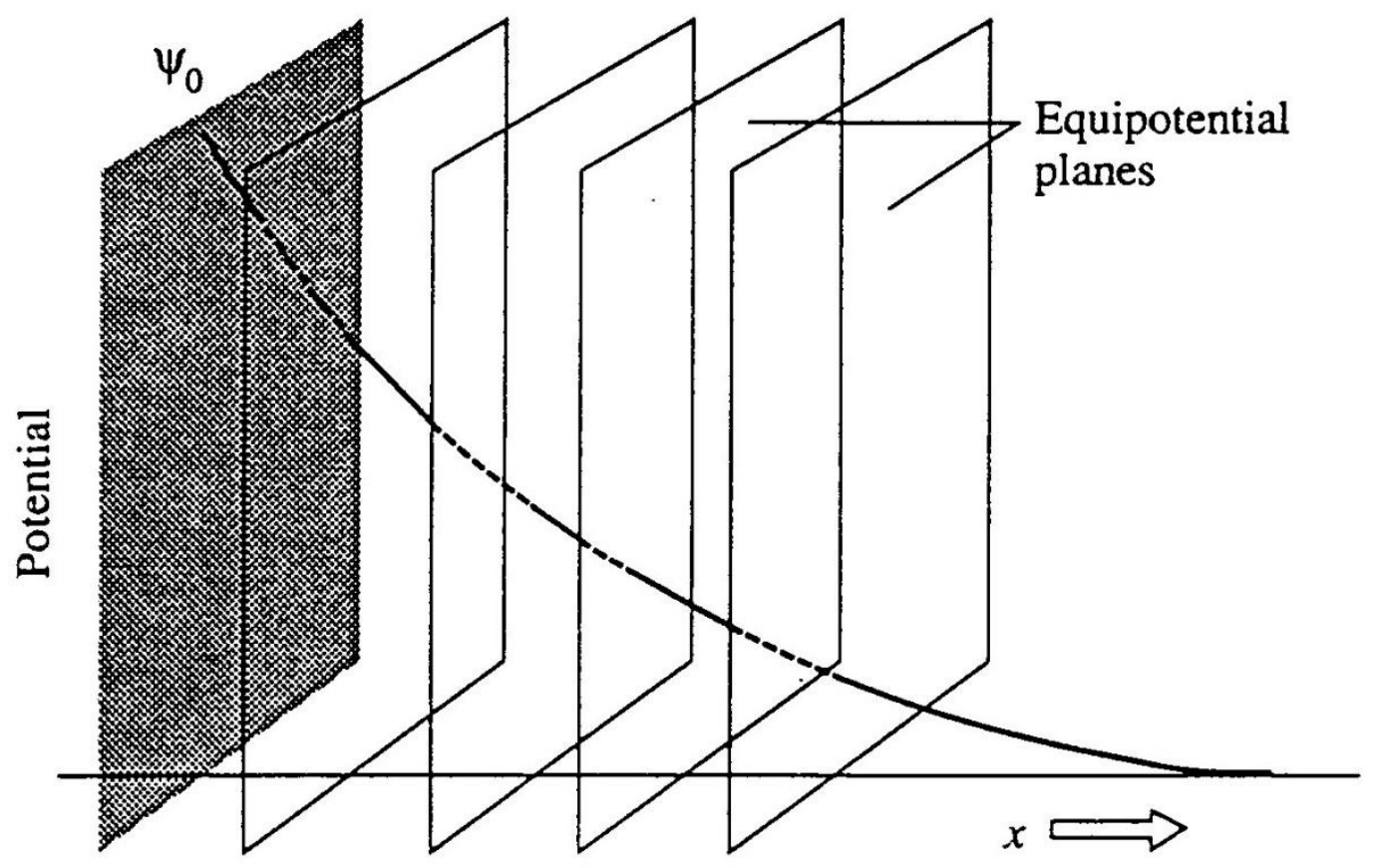

Figure.1. 7. The description of electrical potential with the assumed equipotential in the diffuse part for an example of potential plot with distance. Adept from (Hunter, 1989).

An assumption of equipotential planes is offered as the potential source, 
Figure.1.6. In the equipotential planes, the zeta potential is the same with the same separation to source plane (surface charge). The Debye-Huckel approximation is applied on the Poisson- Boltzmann Equation and the result relates to an assumed parameter $\kappa$ (Debye constant). The parameter $\kappa$ is defined in the relationship of equation.1.4. A plot of the potential vs $1 / \kappa$ (Debye Length) is also result, Figure.1.4 (B) also describes the potential or electrical kinetics distribution across the system.

$$
\kappa=\left(\frac{e^{2} \sum n_{i}^{2} z_{i}^{2}}{\varepsilon_{0} \varepsilon_{r} k T}\right)^{\frac{1}{2}}
$$

Equation.1.4

Experimentally, zeta potential can be measured with different instruments including surface titration and ion exchange (Dina et al., 2001; Lynch \& Dawson, 2008). Other more advanced devices like surface force apparatus (SFA) and AFM can also measure data that can be used in zeta potential calculation methodologies (Barthel, 2008). The Smoluchowski equation is normally used in calculation of zeta potential from measurement of electroosmotic flows (Sze et al., 2003). Based on these calculations, different zeta potential measuring methods were developed for measurement on different sample surfaces. For example, streaming potential was developed for zeta potential measurement at flat surface, such as at separation membranes, and zeta-sizer was developed for zeta potential measurement of colloid particles suspended in aqueous buffers, such as silica beads and bacteria. 


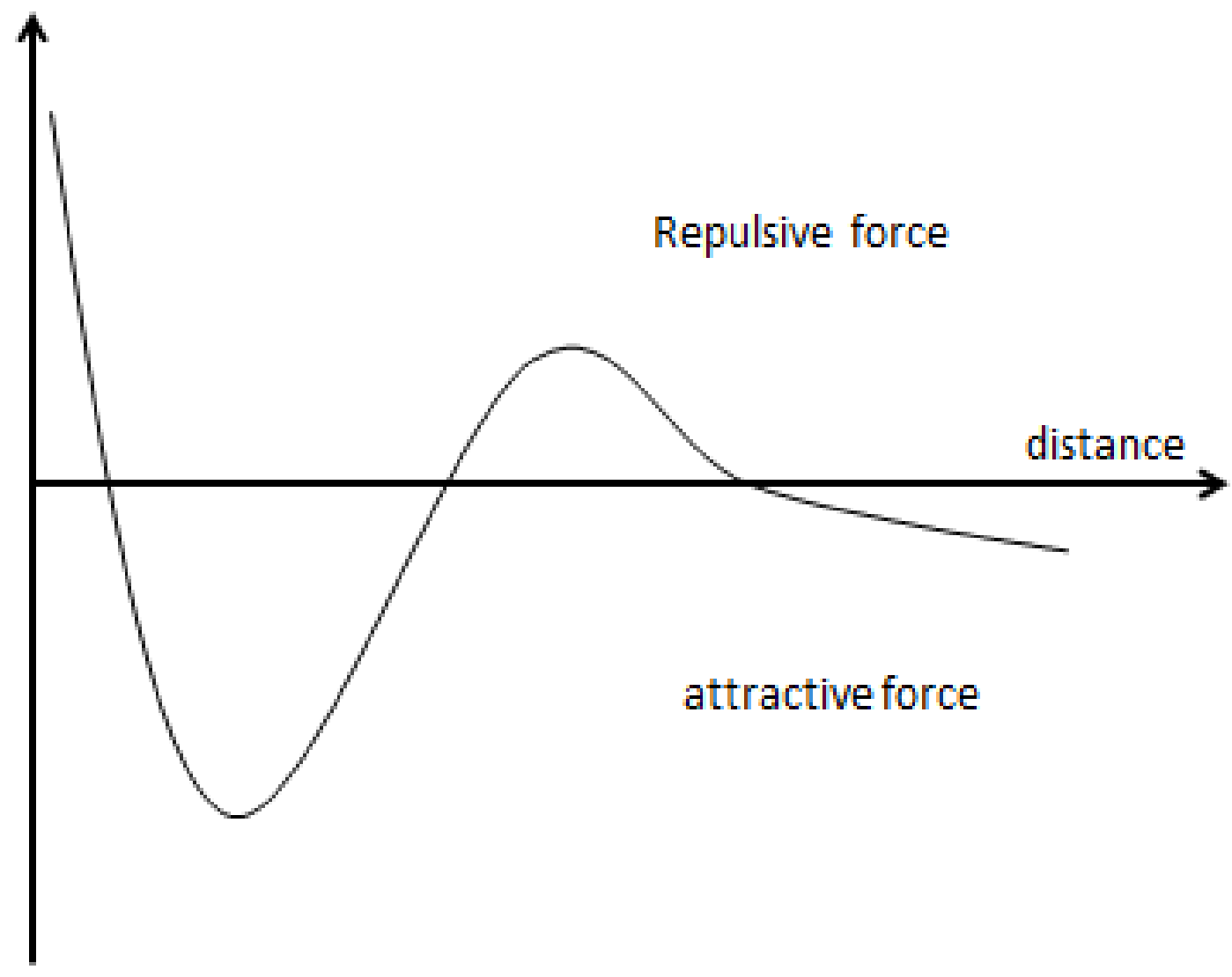

Figure.1. 8. The present of the cooperation effect of repulsive and attractive forces in the system.

In the colloid system, there are also some other important interaction phenomena apart from the electrostatics kinetics, such as Van der Waals forces. A more advanced and comprehensive method to relate the interaction forces was proposed in the Deryaguin-Landau-Verwey-Overbeek (DLVO) theory which is based on the double layer model (Verwey \& Overbeek, 1948). The DLVO theory sums both attractive and repulsive force in interactions (Figure.1.7). The simplified equation for force applied is given as equation.1.5. Where $\mathrm{V}_{\mathrm{A}}$ is the non-retarded Van der Waals interaction, attractive force, and the $V_{R}$ is the repulsive force due to the in double layer.

$$
V_{T}=V_{A}+V_{R}
$$

Equation.1.5

Figure.1.7 approximately presents the two kinds of forces that are applied in the DLVO method, and clearly shows the relationship between force and distance in the diffuse layer adjacent to the surface. In the DLVO theory, the repulsive force of the double layer can normally be described by equation.1.6 (Verwey \& Overbeek, 
1948).

$$
V_{R}=\frac{64 n^{0} k T Z^{2}}{\kappa} \exp (-\kappa D)
$$

Equation.1.6

The mathematical expression of Van der Waals Interaction is more dependent on the method used. If the double layer interaction is approximated as between two flat surfaces still exists, $\mathrm{V}_{\mathrm{A}}$ can be defined as equation.1.7. Where $\mathrm{A}$ is Hamaker constant and $\mathrm{D}$ is distance between molecules.

$$
V_{A}=-\frac{A}{12 \pi D^{2}}
$$

Equation.1.7

Some other techniques are also applied in colloid science to determine DLVO forces, such as osmotic pressure, polymerization, diffusion studies, Langmuir adsorption and. Membrane separation is a filtration process and is an important application of colloid science and surface interaction study thus it is now reviewed.

\subsubsection{Membrane Filtration - a Process Controlled by Colloidal Interactions}

The filtration membrane is important in separation process as an advanced selective barrier for different particles or molecules. Filtration membrane can be classified as microfiltration (MF), ultrafiltration (UF), nanofiltration (NF) and reverse osmosis (RO). Membrane can be made of different materials and structures, such as fibre, polymer or even small molecules of a few hundred Da. Thus, different filtration membranes have different operating properties, such as operating pressure and rejection ratio.

The separation target of membrane processes can be particles or molecules as large as a few $\mu \mathrm{m}$ or as small as a few nm depending on the membrane used. From the size of target, membranes are aimed to separate approximate homogeneous suspension or colloid system. For example, MF can filter colloid particles of a few microns and RO can remove salt molecules from water in desalination processes. Thus, membrane separation can be studied from the perspective of colloid science with characterisation and control focused on DLVO forces to optimise the filtration process. It has been demonstrated that surface electrostatics can influence the membrane filtration in the form of zeta potential (Bellona \& Drewes, 2005). For 
example, low zeta potential occurs fouling on PVDF membranes (Breite et al., 2017; Waite \& Wen, 2011).

In this research, adhesion, electrostatics and surface heterogeneity was studied at membrane surface. Adhesion is related to membrane surface morphology (area of contact) and chemical structures, as well as electrostatics. Many membranes are fabricated from polymer blends using different methods such as phase inversion and casting (Hilal et al., 2015). This blending and different fabrication methods can result in heterogeneity of chemical and morphological properties across the membrane surface. With the study of membrane surface at the micro-/ nano-scale, heterogeneity is an important consideration and can be analysed to assist the understanding of membrane surface electrostatics and adhesion and their impact on membrane separation processes. To achieve the research target, more advanced experimental methods are required. Thus, AFM methods were developed and applied in this study to achieve force measurement and determination of surface heterogeneity with accuracy of $\mathrm{nN}$. As the most important measuring method throughout the whole study, principles and application of AFM are reviewed in the following section.

\subsection{Atomic Force Microscopy}

Atomic force microscopy (AFM) has been applied extensively in the research of colloidal interaction since it was first developed in 1986 ( Johnson \& Hilal, 2015). AFM is a membrane of the scanning probe microscopy family and relies on piezo-ceramic technology for nanoscale positioning. The AFM instrument has key advantages in that it not only allows nanoscale imaging but also force measurement between surfaces within process relevant environments. This has meant that its imaging and force measurement capability has been applied to the study of a wealth of system including separation membranes (Fang \& Duranceau, 2013), proteins (Pfreundschuh et al., 2014), yeast (Marie et al., 2013) and bacteria (Longo et al., 2017). 


\subsubsection{Principles of Atomic Force Microscopy}

Figure.1.8 presents a schematic representation of the AFM instrument. In its simplest mode of operate a cantilever with tip is positioned on top of the sample held on a piezo-ceramic scanner. The tip is systematically rastered across the surface while an optical lever (laser beam and photo-detector) is used to monitor the bending of the cantilever as it encounters changes in surface topography and/or force (Butt et al., 2005). The bending of the lever is then used to generate a 3-D map of the sample surface. In force measurement operation, the cantilever and probe system in raised and lowered in one location and the bending of the cantilever recorded with the incremental extension and retraction of the piezo-ceramic scanner. Hooke's law is then used to convert the bending of the lever to a value of the force applied.

The value of force that is applied to the cantilever-probe system can be quantified by apply the theoretical framework that describes the mechanical properties of the system. The spring constant $\left(\mathrm{k}_{\mathrm{c}}\right)$ can be calculated from the following equations (Braga \& Ricci, 2004; Butt et al., 2005).

$$
k_{c}=\frac{F}{Z_{c}}=\frac{E w t_{c}^{3}}{4 L^{3}}
$$

Where $F$ is force, $Z_{c}$ is deflection of the cantilever at its end, $E$ is Young's modulus, $w$ is width of cantilever, $t_{c}$ is thickness of cantilever, $L$ is length of cantilever and $\rho$ is density. Then, applying the vibration equation of:

$$
v_{0}=0.1615 \frac{t_{c}}{L^{2}} \sqrt{\frac{E}{\rho}}
$$

Equation.1.9

Where $v_{0}$ is resonance frequency of cantilever and $\mathrm{s}$ is surface stress. The resultant equation as the form of:

$$
Z_{c} \approx \frac{4 L^{2} \Delta s}{E t_{c}^{2}}
$$




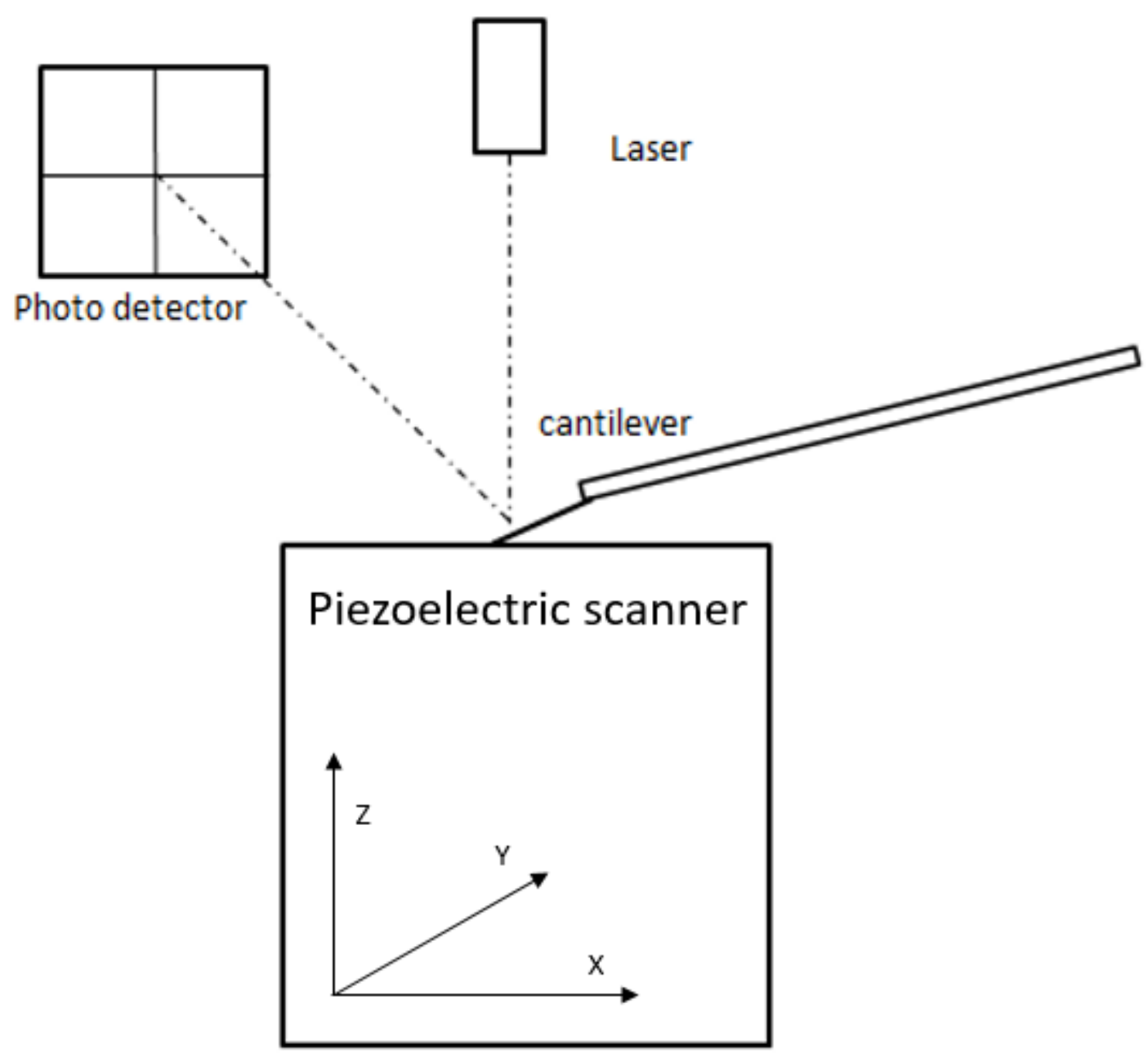

Figure.1. 9. Schematic representation of AFM instrument.

This can be regarded as the mechanical design and basis of the force calculation. The other properties like shape of cantilever, dynamic properties can also be mathematically illustrated. Using the former calculation, a Newton's equation of motion is applied to produce the following equation:

$$
m^{*} \frac{d^{2} Z_{c}(t)}{d t^{2}}+\gamma_{D} \frac{d Z_{c}(t)}{d t}+k_{c} Z_{c}(t)=F(t) \quad \text { Equation.1.11 }
$$

Where $\mathrm{t}$ is time, $\mathrm{m}^{*}$ is effective mass of the cantilever and $\gamma_{\mathrm{D}}$ is damping coefficient. The derivation can be extended for dynamic study, so that the noise power spectrum for a cantilever can be described with a random thermal force by the equation. (Butt et al., 2005) 


$$
v_{O}=\frac{k_{B} t}{\pi m^{*}} \frac{\gamma_{D}}{\left(\omega_{0}^{2}-\omega^{2}\right)^{2}-\gamma_{D}^{2} \omega^{2}}
$$

Equation.1.12

Where $k_{\mathrm{B}}$ is Boltzmann constant, $\omega$ is angular frequency and $\omega_{0}$ is angular resonance frequency of the cantilever. Normally, these calculations are done in the built-in calculation software of the AFM.

\subsubsection{AFM Measurement of Colloidal Interactions}

The major function of AFM that is required in colloidal interaction study is the measurements of force. The force measurement technique can be extended by the creation of a colloid probe where a colloid particle of known size, geometry (sphere) and material is immobilised at the end of the AFM cantilever (Carl \& Schillers, 2008). The colloid particle can then be brought into contact and retracted from a surface to measure the force acting on the particle. The force data can be normalised by dividing by the radius of the particle and the measurement compared with theory and measurements from other techniques.

Many kinds of colloid particles have been used to produce AFM colloid probes for the study of different cell or colloidal interactions. For example, metal oxide microspheres, silica microspheres and polymeric microspheres are commonly used as colloid particle (Johnson \& Hilal, 2015). Butt et al. reviewed colloid probes that are widely used in cell and membrane study including biological materials such as proteins that can be coated on the colloid probe (Butt et al., 2005). Cells can also be glued on the cantilever to create cell probes, the technique was first used by Bowen et al in 1998 who glued a yeast cell on an AFM tipless cantilever (Bowen et al., 1998). Such an approach was used in following studies, for example, the study on colloid probe - yeast interactions by Gaboriaud and Dufrene in 2007 (Gaboriaud \& Dufrêne, 2007).

The use of cell probes is experimentally demanding, thus in the research of this thesis, colloid-membrane interactions and colloid-cell interactions were measured and analysed using colloid probes made with silica beads and hydroxyapatite beads. An alternative approach was used to study the interactions of cells in that instead of 
bringing the cells, as an AFM cell probe, into contact with a surface, the cells were immobilised on a surface and a colloid probe fabricated from the material of interest was brought into contact with the immobilised cells one by one.

In membrane research, membranes are normally studied with filtration experiments to assess the separation parameters (Liang et al., 2014). Measurement of membrane properties at the micro- or nano-scale using AFM has also been done by a few researchers, and has been reviewed by Johnson and Hilal in 2015 (Johnson \& Hilal, 2015). The majority of this research focused on the AFM study of surface roughness and adhesion properties. However, the study of DLVO forces at membrane surfaces has tend to have been restricted to measurement of zeta potential with streaming potential devices (Thomas et al., 2016). Membrane surface adhesion forces were measured using AFM by Lee and Elimelech in 2006 (S. Lee \& Elimelech, 2006), Mi and Elimelech in 2008 (Mi \& Elimelech, 2008) and Liang et al in 2014 (Liang et al., 2014). Electrostatic forces at membrane surface have also been measured using AFM. AFM force measurement and colloid probe technique has also been used to look at particle-particle interactions and the double-layer potential between particles based on analysis of Possion-Boltzmann theory in 2014 (Ruiz-Cabello et al., 2014).

There are a number of colloidal interaction forces that can be studied at cellular surfaces using a range of techniques including AFM, these techniques were reviewed by Aggarwal et al. in 2009 and are summarised as shown in Table.1.1 (Aggarwal et al., 2010). The measurements listed in Table.1.1 shows that normally only one or two kinds of parameters can be measured with these devices.

\begin{tabular}{|l|l|l|}
\hline Bacterial species & Method/technique employed & Parameter measured \\
\hline Denitrifiers & Centrifugation & Adhesive strength \\
\hline Denitrifiers & $\begin{array}{l}\text { Centrifugation and plate drop } \\
\text { method }\end{array}$ & $\begin{array}{l}\text { Tensile strength } \\
\text { shear strength }\end{array}$ \\
\hline P. fluorescens & Micromanipulation technique & Adhesive strength \\
\hline Denitrifiers \& aerobes & Tensile test device & Tensile strength \\
\hline $\begin{array}{l}\text { Mixed culture \& P. } \\
\text { aeruginosa }\end{array}$ & In situ fluid shear variation & $\begin{array}{l}\text { Shear } \\
\text { elastic modulus }\end{array}$ \\
\hline P. aeruginosa & Uniaxial compressive stress & Yield stress \\
\hline
\end{tabular}




\begin{tabular}{|l|l|l|} 
& & elastic modulus \\
\hline Mixed culture & Rotating disk rheometer & Shear modulus \\
\hline P. aeruginosa & Microcantilever method & Tensile strength \\
\hline $\begin{array}{l}\text { Aerobic \& anaerobic } \\
\text { biofilms }\end{array}$ & Micromanipulation technique & $\begin{array}{l}\text { Adhesive shear } \\
\text { strength }\end{array}$ \\
\hline Mixed species biofilm & Fluid dynamic gauging & $\begin{array}{l}\text { Cohesive shear } \\
\text { strength }\end{array}$ \\
\hline Undefined mixed culture & Atomic force microscopy & $\begin{array}{l}\text { Cohesive shear } \\
\text { strength }\end{array}$ \\
\hline S. epidermidis & $\begin{array}{l}\text { Microcantilever method for } \\
\text { intact biofilms }\end{array}$ & Cohesive energy \\
\hline
\end{tabular}

Table.1. 1. Review of cell interactions measurements without different techniques by Aggarwal et al. in 2010, list of cells tested, techniques used, parameters measured and first published paper, adept to (Aggarwal et al., 2010).

Examples of research that has used AFM to study colloidal interactions include the work of Wilhelm et al. who studied the interaction between Hela tumor cells $\backslash$ Mouse RAW macrophages and iron oxide nanoparticles in 2002 (Wilhelm et al., 2002), Shukla et al. studied the influence of gold nanoparticles on RAW264.7 macrophage cells in 2005 (Shukla et al., 2005) and Limbach et al. studied the exposure of silica nanoparticles to human lung epithelial cells in 2007 (Limbach et al., 2007). These studies were more focused on the adsorption and influence of these particles on the behaviour of the cell surface instead of interaction forces. Most of the forces measurement that presents in Table.1.1 is not from an AFM. However, AFM are of interest by more researchers compare to these devices used in Table.1.1. For example, the study and analysis of interaction forces between cell and colloid particles by Vasir and Labhasetwar in 2008 is majorly based on AFM imaging and force measurement from colloid probe (Vasir \& Labhasetwar, 2008). Repulsive and attractive force have also been measured by AFM for the fibroblast cell-line L929 with silica colloid probe (McNamee et al., 2006). A review of AFM interaction measurement between colloid probe and a cell/membrane surfaces is presented in Table.1.2. The study of cells using AFM was also reviewed by Wright et al. in 2010, who discussed AFM measurement of cell mechanics, interaction forces of biofilms 
and single cells. The potential of AFM combined with other characterization methods was also reviewed (Wright et al., 2010).

\begin{tabular}{|c|c|c|c|}
\hline sample & probe & Force & Reference \\
\hline 3A9 cell line & LFA-1/ICAM-1 & adhesion force & $\begin{array}{l}\text { (Wojcikiewicz, } \\
\text { Zhang, \& Moy, } \\
\text { 2004) }\end{array}$ \\
\hline HEK293 & DNA & $\begin{array}{l}\text { unbinding } \\
\text { force }\end{array}$ & (Han et al., 2005) \\
\hline M.bovis.BCG & heparin & adhesion force & $\begin{array}{l}\text { (Gaboriaud \& } \\
\text { Dufrêne, 2007) }\end{array}$ \\
\hline P. aeruginosa & $\begin{array}{l}\text { silica bead with } \\
\text { biofilm }\end{array}$ & adhesion force & (Lau et al., 2009) \\
\hline mica & silica & adhesion force & (Zhang et al., 2014) \\
\hline E. coli & $\begin{array}{l}\text { grephene oxide } \\
\text { functionlized }\end{array}$ & $\begin{array}{l}\text { adhesion force } \\
\text { interaction } \\
\text { force }\end{array}$ & $\begin{array}{l}\text { (Castrillón et al., } \\
\text { 2015) }\end{array}$ \\
\hline hematite & E. coli & adhesion force & (Zhang et al., 2011) \\
\hline polymer membrane & $\begin{array}{l}\text { humic acid } \\
\text { functionlized }\end{array}$ & adhesion force & $\begin{array}{l}\text { (Johnson et al., } \\
\text { 2015) }\end{array}$ \\
\hline
\end{tabular}

Table.1. 2. Representative researches based on colloid probe and AFM force measurement from 2004 to 2015, type of both contact surfaces, force measured and authorizer are listed in table.

\subsection{AFM Colloidal Interaction Study - the Research Gap}

The review in section.1.3.2 shows that using AFM in conjunction with a colloid probe many kinds of interactions can be measured, for example, adhesion (Lau et al., 2009), unbinding force (Han et al., 2005), and electrostatic interactions (Ruiz-Cabello et al., 2014). Thus, the large potential of AFM's application on membrane and cell interaction studies has been indicated.

An interesting application for AFM force spectroscopy is measuring the zeta potential on membrane or cell surfaces. Researchers have established calculation methods for zeta potential with mathematical simulation of force-distance curves based on extended DLVO theories (Brant \& Childress, 2002; Brant \& Childress, 2004; Brant et al., 2006). This then permits the measurement of zeta potentials with AFM 
when these mathematical simulations are compared to AFM force-distance data. Recently, a model that calculates double layer potentials between two latex particles was built based on Possion-Boltzmann theory by Ruiz-Cabello et al. in 2014 (Ruiz-Cabello et al., 2014). A calculation of zeta potential based on simulation of AFM force curves in different boundary condition was also achieved in the study by Bowen et al. in 2002 (Bowen et al., 2002). With the promise of these developed models and DLVO theories, the calculation of the zeta potential at more complicated surfaces can be achieved. So far, direct calculation of zeta potential from AFM force curves at membrane or cell surfaces has not been developed.

Hydrophobicity, chemical forces and electrostatic forces at biological surfaces are related to each other (Gaboriaud \& Dufrêne, 2007). In a colloid particle drug delivery study by Ojewole et al. in 2008, drug release kinetics were also found to be influenced by these parameters (Ojewole et al., 2008). Chemical kinetics theory is also directly used in the interaction study by Wilhelm et al. in 2002 that studied the surface interaction from reactions kinetics on cell surfaces (Wilhelm et al., 2002). Thus, in the present study it is necessary to analyse hydrophobicity, chemical bonding and electrostatic forces together. While, chemical bonding forces can be related to reaction kinetics or reaction equilibrium. Therefore, a further research gap which this thesis attempts to fill is the combination analysis of hydrophobicity, chemical forces and zeta potential to greater understand the behaviour of colloids at membrane and cell surfaces.

With the application of AFM, it becomes achievable to study single cells at the nanoscale (Wright et al., 2010) and similarly to study the phenomena that control membrane separation processes at the nanoscale (Johnson \& Hilal, 2015). A lot of previous research using the AFM force measurement capability has focused on the study of one aspect of the force interaction. Particularly, an average adhesion force missing the opportunity to deconvolve contribution interaction forces from AFM force curves by manipulation of environmental conditions and corroboration from a force curve in combination with other techniques, such as hydrophobicity measurement, and statistical analysis; a lot of research measures an average force using AFM but 
does not consider the range of the measurement which can be viewed as a characterisation parameter. For example, in the case of surface heterogeneity at membrane surfaces can indicate differences is polymer blending during fabrication of the membrane surface (Hilal et al., 2015). For cells, heterogeneity of forces measured at cellular surface could indicate a greater range of protein expression at the surface (Muller \& Dufrene, 2011). A further tool in the AFM tool box which has not been exploited extensively is the instruments ability to map forces across a surface, thus presenting the opportunity for spatial resolution of the heterogeneity of forces such as electrostatic repulsion across a surface.

\subsection{Aim and Objectives}

The preceding research gap analysis has identified that there is great potential for the application of AFM force measurement to the study of colloidal interactions that control the behaviour of many important system such as industrial processes exemplified by membranes used in separation processes and biological processes as exemplified by cell-particle interactions. Thus, the aim of the present study is to extend the force analysis of AFM by closer examination of force-distance curves measured between a colloid probe and surfaces. This will be achieved by using DLVO theories to interpret force data and a statistical framework. To achieve this the present study examines the interaction of two systems namely membrane separation and microbial cell interactions. The following objectives to meet this aim have been identified.

The thesis establishes a theoretical framework and model that can be used calculate zeta potential from AFM force curves; FORTRAN code was constructed for processing AFM force-distance raw data and calculating parameter such as maximum adhesion force, adhesion break-off distance and zeta potential (Appendix.2). The model should be validated to provide confidence in the approach and measurement of zeta potential and this is achieved within Chapter III Of the thesis, which examines 
the exemplar system of particles interaction with a membrane that is used in separation processes. To provide a comparison of zeta potential during the modelling and method validation, zeta potential was measured with other methods that include Zeta-sizer (Zetasizer, n.d.) for microbial cells and streaming potential for membrane surface (Fievet et al., 2001). Once the method was validated for membrane research, further application is demonstrated by the study of microbial cell interactions and their control by environmental additives.

The extension of AFM force analysis was further demonstrated by the measurement of zeta potential across a surface using AFM force-measurement in conjunction with the Mann-Whitney U test (MWW) and Gaussian distribution on both microbial cell and membrane surfaces. The influence on interaction forces of different buffer $\mathrm{pH}$, ionic strength and bioactive chemicals were studied and analysed with the zeta potential calculation model and statistical analysis.

A further supporting technique was also established for the study of microbial cell hydrophobicity based on hydrophobic partitioning within different solvents. A model that focused on reaction equilibrium and Gaussian distribution was developed to link the hydrophobicity to an estimation of cell surface hydrophilic chemical groups activity. This was then used to analyse the relationship between hydrophobicity, chemical bonding and zeta potential from AFM force-distance curves.

With the aim and objectives identified, the research structure can be designed as a combination of literature research, experimental measurement, mathematical modelling and analysis. Therefore, the research structure can be built as presented in Figure.1.9 to facilitate the overall study of the thesis. 


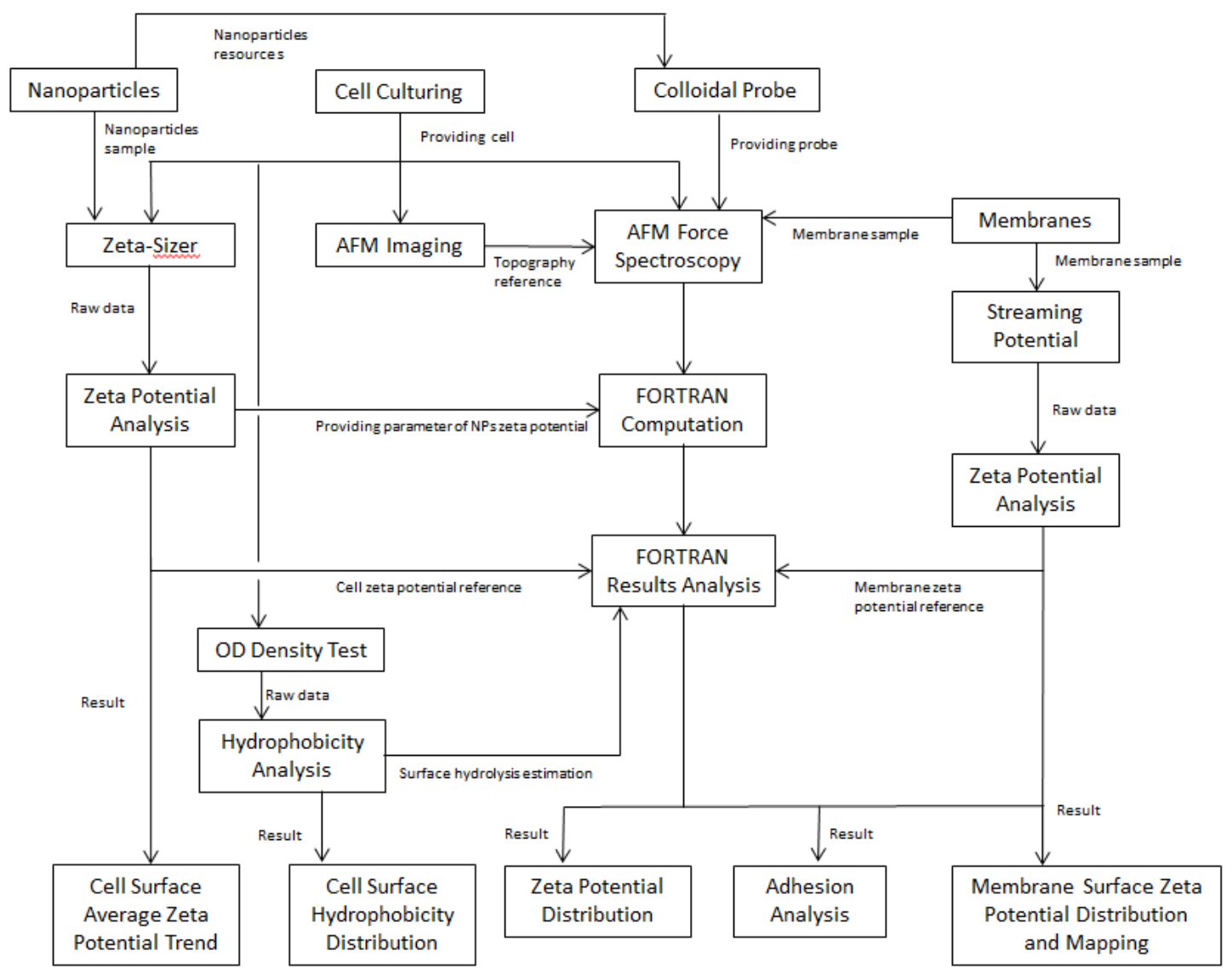

Figure.1. 10. A diagram showing cooperation between different experiments and analysis. 


\section{Chapter II Materials and Methods}

\subsection{Introduction}

This chapter outlines the experimental methods and tools that are used in Chapter IV - V and reviews the calculation methods and software that was used in model development in Chapter III.

\subsection{Experiments Protocols and Preparation}

\subsubsection{Materials}

All chemicals were supplied by Fisher Scientific unless otherwise stated. All suspension and solution were prepared using deionised water (DI). When required $\mathrm{pH}$ adjustment was achieved by the addition of $1 \mathrm{M} \mathrm{NaOH}$ or $\mathrm{HCl}$.

\subsubsection{Cell Culture}

Yeast cells and Gram negative bacterial cells were used as model cell systems within this study. The yeasts, NCYC-1324 and NCYC-1681, were provided by national collection of yeast cultures. The Gram-negative bacterium of Pseudomonas fluorescens was provide by Dr. Bob Lovitt (Swansea University). Growth media were previded by Oxoid Thermo Scientific. MYGP agar plates were used to culture the yeast cells (as shown in Table.2.1), nutrient agar plates made with 28g/1 of commercial nutrient agar powder dissolved in DI water. Media was sterilised by autoclaving (Priorclave) for $15 \mathrm{~min}$. After pouring plates were sealed with para-film and sterilized with UV light within a biological safety cabinet class II (Astec Microflow). Plates were then stored prior to use in a fridge and for a maximum of 2 weeks. Liquid cell culture was prepared in the same way but with the omission of agar. The flasks were sealed prior to autoclaving with cotton wool and foil.

Cell plates were refreshed every week with streaking to single colonises using aseptic techniques. Flamed loops were used to select single colonies that were then 
used to inoculate fresh agar plates or liquid cultures. Inoculated media flasks were resealed again and cultured in an incubate shaker (New Brunswick Scientific) at $150 \mathrm{rpm}, 25^{\circ} \mathrm{C}$ for 24 hours.

\begin{tabular}{|c|c|c|c|c|c|}
\hline Formula & Yeast extract & Malt extract & Peptone & Dextrose & Agar \\
\hline $\begin{array}{c}\text { Concentration } \\
(\mathrm{g} / \mathrm{l})\end{array}$ & 3 & 3 & 5 & 10 & 20 \\
\hline \multicolumn{6}{|c|}{$\mathrm{pH} 6-7$ at $25^{\circ} \mathrm{C}$} \\
\hline
\end{tabular}

Table.2. 1. Ingredient of MYGP agar, formula in the concentration of $\mathrm{g} / \mathrm{l}$ in water-based solution.

\subsubsection{Zeta Potential Measurement}

A Zeta-Sizer (Malvern instruments) was used for zeta potential measurement. Zeta-Sizer cells of DT-1060 and its replacement DT-1070, both supplied by Malvern instruments, were used for zeta potential and particle size measurement. Yeast, bacteria and colloidal particles were washed and suspend in $1.5 \mathrm{ml}$ of DI water. $20 \mu \mathrm{l}$ of the washed suspension was then diluted in $1.5 \mathrm{ml}$ of buffer prior to zeta potential testing. The buffer solutions used were $\mathrm{NaCl}$ solutions at $0.1 \mathrm{M}, 0.01 \mathrm{M}, 0.001 \mathrm{M}$ and DI water, each concentration was measured at $\mathrm{pH} 5,7$ and $9.1 \mathrm{ml}$ of the diluted particle suspension was loaded into the Zeta-Sizer cell. It was important that samples were freshly prepared and used within 20 minutes as the particle suspension were not stable. Air bubbles must be precluded from the Zeta-Sizer cell and it is sealed with therom plates (from Malvern Instruments) and then loaded into the Zeta-Sizer for test.

120 seconds were allowed for the apparatus to warm up the sample suspension to $25^{\circ} \mathrm{C}$. Size of samples were measured with 10 s measuring time and 20 repeats over 3 runs. Zeta potential was measured with 11 runs each with 10 measurement repeats. Zeta potential and particle size were recorded for each run as the average of all repeats included in the run.

After use, the Zeta-Sizer cell was immediately washed. Wash with DI water, it was then sterilised with $70 \%$ vol ethanol to kill the microbes. The Zeta-Sizer cells were then rinsed with DI water again to remove the ethanol residual and then left to 
dry thus ensuring that the zeta-sizer cell is clean and dry for next use.

\subsubsection{Hydrophobicity Determination}

The hydrophobicity was measured using hydrophobic partitioning in different buffer as determined by optical density in a method adapted from bacteria adherence to hexadecane (BATH) test (Williams \& Fletcher, 1996). A spectrophotometer (UVmini-1240 UV-VIS from Shimadzu Scientific Instruments) was used to measure optical density (OD) at a wavelength of $660 \mathrm{~nm}$. OD difference of the different solvent partitions is used as the index of particle density difference, which is related to hydrophobicity. Hexadecane was used as the organic solvent and DI water as the inorganic solvent.

For microbial cells, $1.5 \mathrm{ml}$ of the culture was washed and suspended in $5 \mathrm{ml}$ of DI water at $25^{\circ} \mathrm{C}$ in a $15 \mathrm{ml}$ test tube. The sample was stirred for 30 seconds then $3 \mathrm{ml}$ of the suspension was placed in a cuvette and the OD measured; this was referred to as OD1. The $3 \mathrm{ml}$ sample was then returned to the test tube, as followed by 30 seconds mixing. $0.5 \mathrm{ml}$ of hexadecane was then pipetted into the test tube which was then mixed for 1 minute so that both liquids fully contacted each other. 5 minutes were then applied for the hexadecane and water to separate. The floating hexadecane was then removed and OD of the water partition determined; this was referred to as OD2. Thus, the index of particle density difference and the measure of hydrophobicity is provided by the percentage OD2/OD1 (X100\%).

The washed water partition was regarded as without hexadecane but there may still have been microscopic size hexadecane droplets. A time dependent test of OD error caused by hexadecane was done to account for any hexadecane remaining. $0.5 \mathrm{ml}$ of hexadecane was mixed with $5 \mathrm{ml}$ DI water for 1 minute. The system was then allowed to partition for 5 minutes. The hexadecane was removed and $3 \mathrm{ml}$ of the water suspension placed in the spectrophotometer and the OD was recorded every 1 minute. The measured influence was plotted (Appendix.1.1) and can be directly used to remove the error induced by residual hexadecane; the OD at 5 mins was subtracted 
from OD2.

5 minutes is the maximum separation time allowed for hexadecane separation due to the cell aggregation in this buffer. A time dependent cell aggregation error test was required to analyse the error caused by cell aggregation on the OD. The suspension was shaken for 30 seconds and then placed in the spectrophotometer. OD was measured each minute as in the hexadecane error test and plotted as to remove the error caused by cell aggregation (Appendix.1.2 and 1.3); the OD at 5mins was also subtracted from OD2.

\subsubsection{Atomic Force Microscopy}

\subsubsection{Atomic Force Microscopy Sample Preparation}

Sample preparation is very important in AFM analysis to ensure consistency of both imaging and force distance techniques. The different methods used for the preparation of separation membranes and microbial cells are now discussed.

\subsection{Membrane Sample Preparation}

Two kinds of membrane were analysed with AFM; DK membrane an nanofiltration membrane and Cyclopore microfiltration membrane. The membranes were soaked in DI water for more than 12 hours to remove any preservation fluid layers and to hydrate the structure (Oatley et al., 2012). Membranes were cut into a square shape with side length of 1-2 $\mathrm{cm}$ and attached to the AFM sample holder using water-proof double sided sticky tape that had been cut to size. The double side tape must be fully covered to ensure no material leeched into the AFM analysis system. The non-adhered edge of the membrane was cut off, so as not to be displaced in liquid and interfere with AFM. 


\subsection{Cell Sample Preparation}

The cultured microbial cells were dispersed and fully mixed within the growth media. A process to wash the microbial cells was adopted to reduce residual media coating the cells. $1.5 \mathrm{ml}$ of cell culture were placed in an eppendorf (Eppendorf Biotech Company) centrifuged with a mini centrifuge (Thermo Scientific) at 3000rpm for 3 minutes. The supernatant was then removed and the cells deposited at the bottom of centrifuge tubes were resuspend in DI water and centrifuged again. This washing of the microbial cells was repeated and the cells finally resuspended in DI water.

Both yeast and bacterial cells are difficult to immobilise on either glass or metal without surface functionalisation. Thus, the present study used a technique suggested by (Touhami et al., 2003) to capture cells at a micro filtration membrane surface, this would immobilise the cells with enough strength to resist the mechanical movement of the AFM cantilever during imaging and force measurement. A suspension of cells was filtered through a Cyclopore micro filtration membrane of pore size close to the diameter of the microbial cells. The membrane was then washed to remove any weakly attached cells that could contaminate the experiment buffer solution and interfere with the AFM laser monitoring the deflection of the cantilever. The membrane was then attached to the AFM using the protocol described above.

\subsubsection{Colloid probe}

A micromanipulator (Singer Instruments) was used to prepare the AFM colloid probes. The colloidal particles were silica beadsv (Polysciences, Inc), and hydroxyapatite beads (Sigma) and were attached to AFM contact cantilevers (DNP-10 Bruker). The AFM chip has 4 cantilevers, two cantilevers on each side (Figure.2.1b). An optical microscope was used to select a suitable cantilever for the colloid probe and glass glue (Loctite) was used to attach the particles on the cantilevers.

Colloidal particles and glass glue were placed on opposite ends of the same 
microscope slide within the micromanipulator. A small amount of glue was then picked up by the cantilever housed at the end of a micromanipulation tool. The colloid particle was then selected using the microscope of the micromanipulator and picked up by the glue on the cantilever.

This process repeatedly produced colloid probes optimally located on the underside at the apex of the cantilever (Figure.2.1 a). This technique has produced very high quality AFM colloid probes with a minimal amount of glue (Bowen et al., 1998). The fabricated colloid probe was removed from the micromanipulator and stored in a dry and clean atmosphere for 24 hours in order for the glue to dry prior to use.

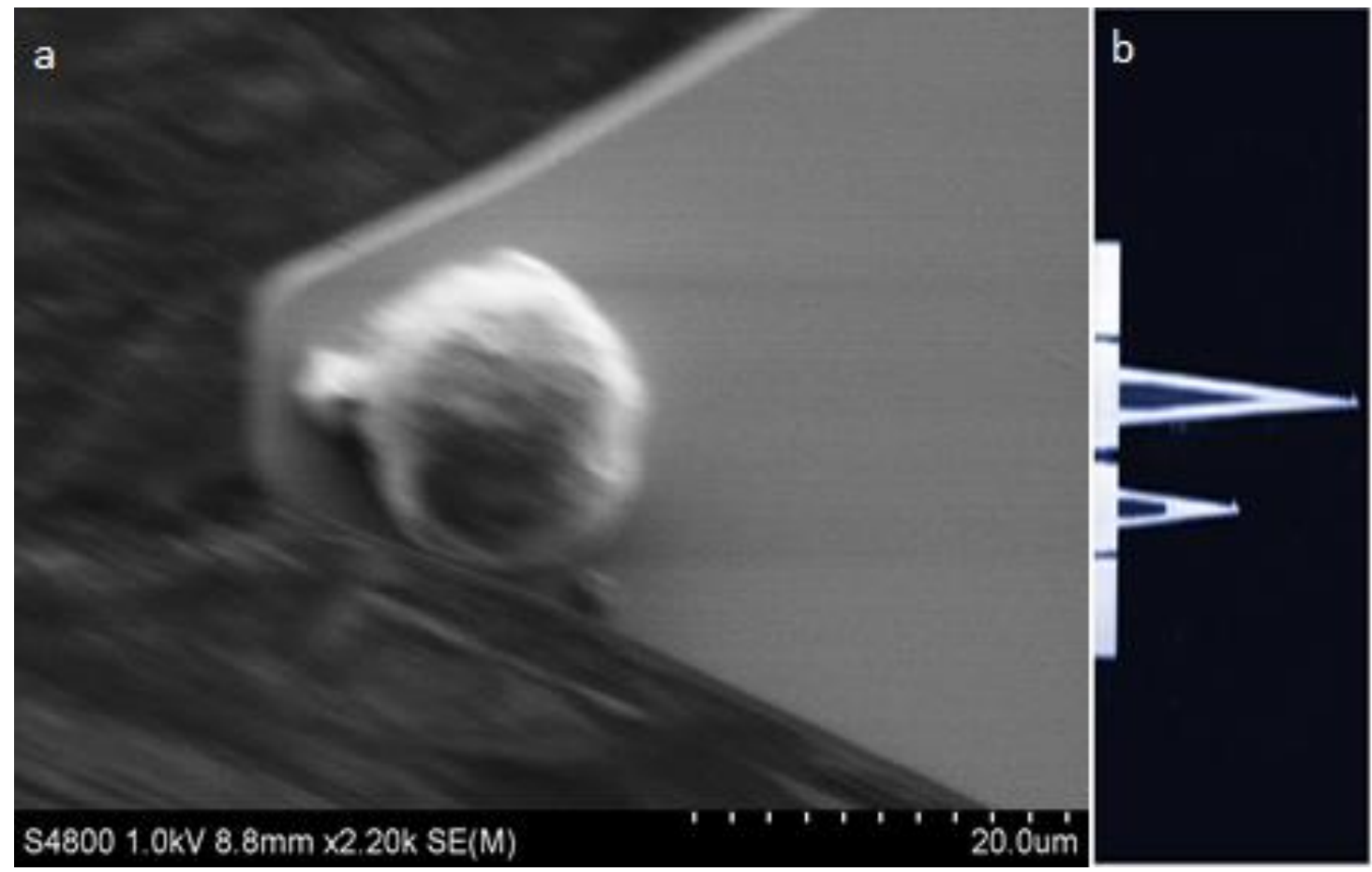

Figure.2. 1. SEM imaging of the attached silica bead on colloid probe (a) and the provided DNP-10 contact cantilever (b) illustration provided by manufacturer of buker AFM probes.

\subsubsection{AFM Imaging}

The AFM instrument required to be set up before use. Due to the working principle of AFM, a laser beam must be reflected of the back of the gold coated cantilever and onto a position sensitive photo diode. Two kinds of AFMs were used in this study for sample surface imaging and force measurements, Park XE-100 AFM 
(Park Systems), and JPK Nano-wizard AFM (JPK Instruments). Preparation of these two different AFM instruments is different due to differences in their configuration however the work with the same principle.

The imaging of sample surface was taken with different procedures between contact mode and non-contact mode. Contact mode was applied in this study, as contact mode cantilever are softer, which make it better in force measurement. The cantilever is scanning the surface at either $\mathrm{X}$ or $\mathrm{Y}$ direction. Normally, $\mathrm{X}$ direction is the random line scan direction. Y direction is divided into 512 points as the random set of both AFM. The image is made up by 512 lines at X direction. The accuracy of scanning can be adjusted by select how many lines to be scanned in the image. 512 lines were used for all the AFM imaging either contact mode or non-contact mode. Two-way scanning was used on Park 100 AFM and JPK Nano-Wizard AFM. Low cantilever line scanning frequencies were used on AFMs, 0.8-1.0 Hz for Park 100 AFM and 0.6-0.8 Hz for JPK Nano-Wizard AFM. Set point and P-gain were left as random. These values were not effecting the scanning significantly.

For the testing of membranes, $10 \mu \mathrm{m}$ of scanning size was used to provide a clearer surface topography of membrane. The cell sample was immobilized on a membrane surface, it may result no cell scanned if scale set to small. Maximum scale of $50 \mu \mathrm{m}$ for JPK Nano-Wizard AFM was used for cell testing. Rough surface may also interrupt the imaging. Scanning may be in a low precision or stop in a large surface roughness.

\subsubsection{AFM Force Measurement and Mapping}

Contact mode imaging will provide a topography scan of the surface to help select the point for force testing. Park -AFM 100 AFM was used for membrane test JPK Nano-Wizard AFM was used for cell test. Force spectroscopy was turned on to ready the cantilever for force measurement. DNP-10 (Bruker) cantilevers were used for force measurement. The spring constant $(0.12 \mathrm{~N} / \mathrm{m})$ specified by the manufacturer was 
used. There are many methods available for measuring the spring constant. These include methods based on the material and geometrical properties of the cantilever, monitoring the change in the resonant frequency of the cantilever upon addition of known weights (Cleveland et al., 1993), calibration against a cantilever of known spring constant during a force curve (Torii et al., 1996) and thermal vibration (Levy \& Maaloum, 2002). These methods can be subject to 10-20\% error (Green et al., 2004). The spring constant quoted by the manufacture is often based on cantilever dimensions which can be sensitive to defects and differences between manufacturing batches. The present study found that measurement of the spring constant using the thermal method of the JPK instrument yielded values within 5\% of that provided by the manufacturer. Thus, to save time the present study used the nominal spring constant specified by the manufacturer.

JPK Nano-Wizard AFM was used for the force curving on cell samples. Because the force curving position can be adjusted on scanning screen for JPK Nano-Wizard AFM compare to Park-100 AFM. The property of cell sample is cells randomly distributed on the membrane, uncertain distribution of cells requires flexibility tapping position on sample surface. Set point and P-gain were used as random set. AFM trace down speed are set by the tracing time and set traveling distance of cantilever. Recording points of force curve is also depending on the traveling time of cantilever. Fast speed of 0.5 second tracing time will lead to 512 points of force curve, higher tracing time could cause more points recorded on the force curve. Signal measure model was used on the force measurement.

Set point is the maximum force that can apply to cause the cantilever bending, random set was set for Park-100 AFM. Park-100 AFM is lack of control of force measurement. Measurement point selection is not available for force measurement, it only tapping on the centre of topography scan. Random set of 512 recording points of a single trace or retrace is applied on the force curve. Cantilever trace down speed was set as either $0.15 \mu \mathrm{m}$ or $0.30 \mu \mathrm{m}$ for Park 100 AFM. Force mapping is based on the force curving. Automatic selection of tapping points was selected on the image. A matrix was used on the point selection. The matrix will contain same columns and 
rows of points. $4 \mathrm{X} 4,6 \mathrm{X} 6,8 \mathrm{X} 8,10 \mathrm{X} 10$ and $12 \mathrm{X} 12$ of matrix models were available as selection(JPK Instruments, 2009). 4X4 matrix was used for the mapping of membrane samples.

\subsection{Data Treatment}

AFM data treatments was based on the DLVO theory and AFM operating principles. Zeta potential, adhesion force between probe and sample were the target results for the analysis. The analysis processes are now discussed and the subsequent method validation is described in Chapter III.

\subsubsection{FORTRAN Programming and Analysis of Force Curve}

FORTRAN programs were used to analysis the raw-data provided by the AFM force measuring. FORTRAN codes are present as Appendix.2. The program is used to estimate the surface charge of colloid probe for further FORTRAN programs calculation. Calculation process of FORTRAN AFM force curve analysis programs are working as the flow chart, Figure.2.2. Principally, the programs for either Park or JPK are all the same, there will be slightly different based on the default data saving setting difference of Park and JPK control software. Both Park XE-100 AFM and JPK nano-wizard AFM force curve analysis is under the same analysis protocol. Data for each single program was saved in a txt file for each curve.

Mathematical adhesion calculation model based on JKR and DMT were involved in the data treatment (Barthel, 2008). It provides a simple transfer of forces to energy in a linear relationship. Through the developing of the JKR and DMT model are separately identical for adhesions caused by non-contact or contact surface adhesion (Barthel, 2008). Therefore, the point of separate can be regard as the point of calculation model change. 


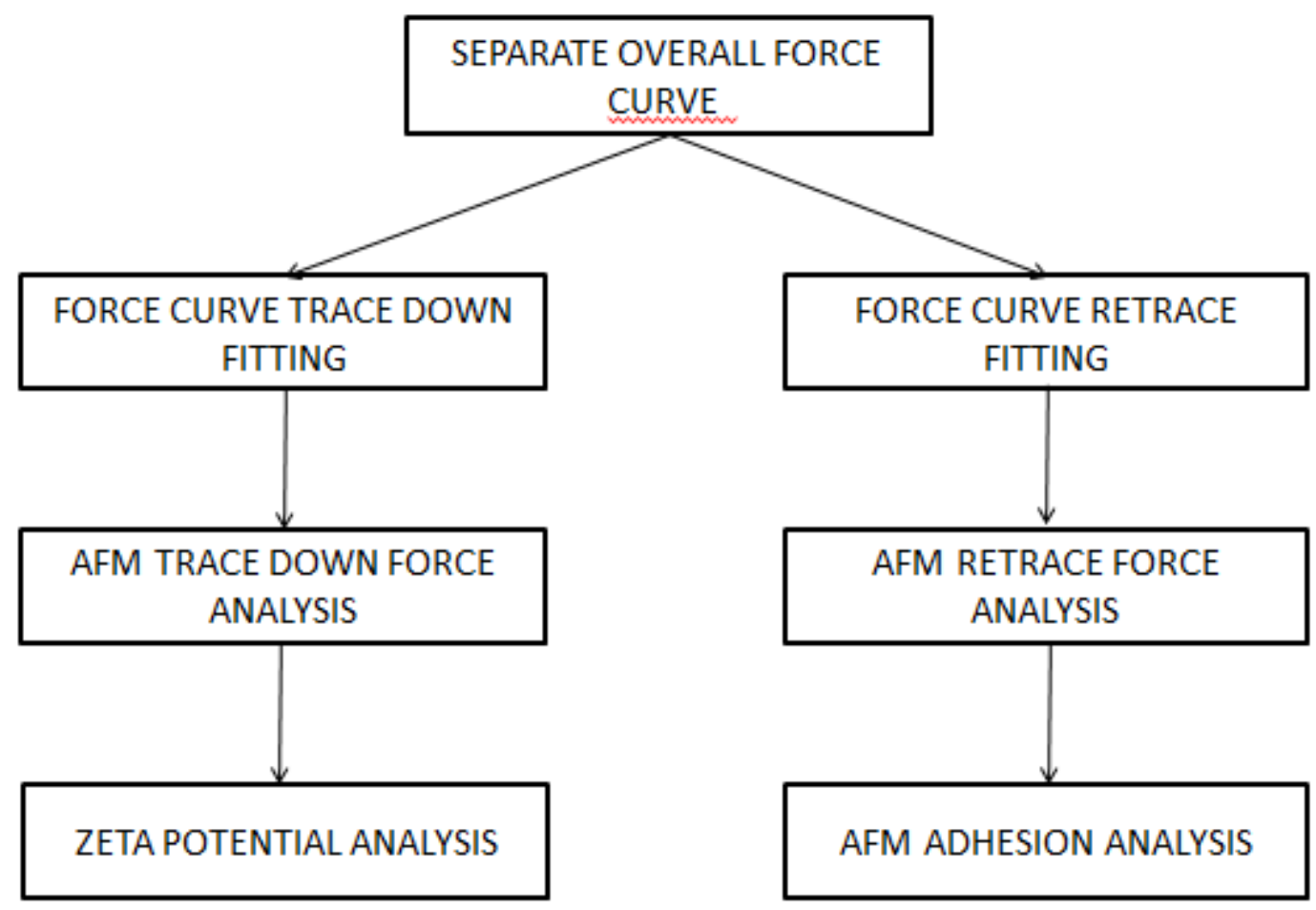

Figure.2. 2. Figure of name and cooperation of the FORTRAN code programs involved in the computation based numerical analysis model.

The FORTRAN analysis of AFM force curves for surface zeta potential is based on a calculation of force related potential between two charged surfaces, the colloid particle on colloid probe and sample surface. The colloid probe has to be measured with zeta-sizer as a more general reference of computation of sample zeta potential. The experimental protocols are shows in Chapter.2.2.2. The zeta potential of the two kinds of colloids used, silica bead and hydroxyapatite bead are measure with zeta-sizer for hydroxyapatite and silica bead before the application of calculation model on AFM force curves.

The FORTRAN program groups can also be rewritten and accomplish the same computation propose in a single program with several subroutines simulate the functions of different programs in the codes of the programs cooperation model. There is an advance of the aggregated program. All the supporting calculations are able to apply in the program, not only the two main lines of computation processes. 
From the calculation structure introduction state in Figure.2.3, the supporting calculation of surface charge and surface bending effects can also be calculated and automatically applied for the final result estimation.

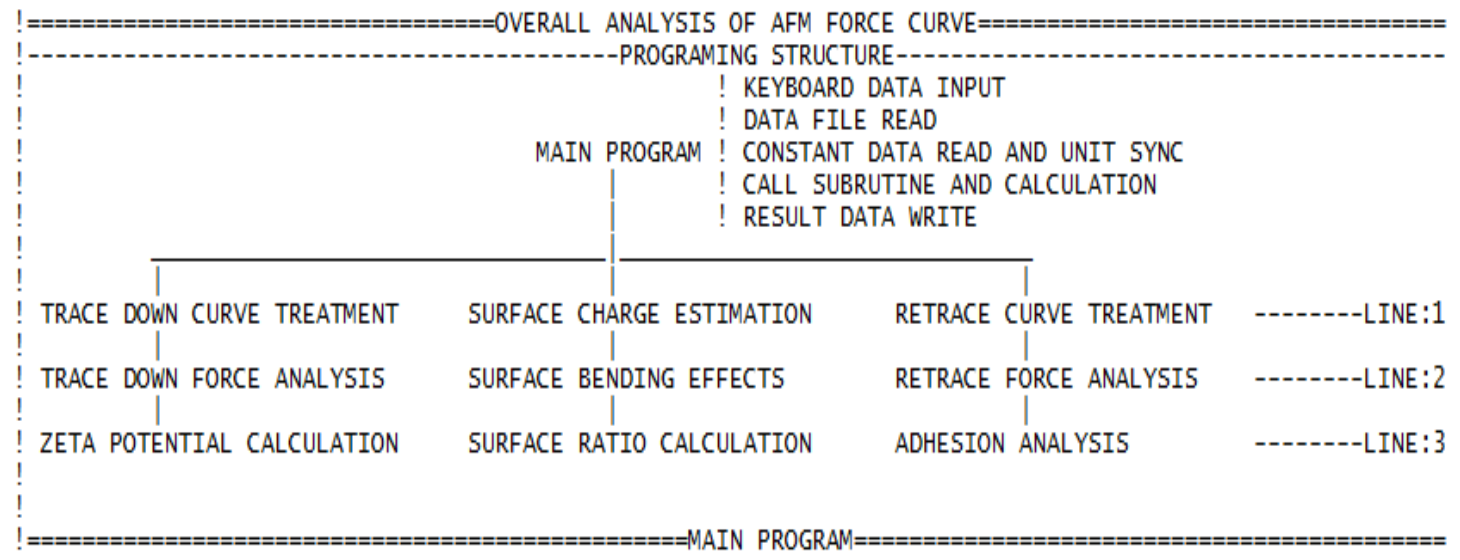

Figure.2. 3. The introduction headline of the combined FORTRAN program, introduction of the whole function and their linking in the calculation model.

\subsubsection{Statistical Analysis}

Statistical analysis used a Mann-Whitney U test (MWW) to examine the significance difference between data sets. MWW is a mature model developed to compare two data sets, which meets the statistical analysis requirement of data in this study. Heterogeneity of the data sets was analysed in Gaussian distribution context. Both these sets of analysis were achieved through the establishment of an Excel spreadsheet platform which processed FORTRAN data derived from the original AFM force curves. The development and application of the data analysis is described in more detail as part of the method validation in Chapter III. 


\section{Chapter III Model Development}

\subsection{Introduction}

There are many theories that can be applied in the force analysis of this study, principle of which is the DLVO theory. The DLVO theory enables the analysis of the surface electrostatic interactions using the force-distance curve measured from by AFM spectroscopy. The electrostatic forces only become significant when two charged surfaces are as close as a few $\mathrm{nm}$. There are a number of parapeters that must be identified and defined from force curves during their analysis using the DLVO theory. For example, definition of the surface contact position on force curves becomes an important consideration in the application of the DLVO theory for the calculation of zeta potential, which is major objective of this study. To facilitate the interpretation of force curves within the theoretical framework of the DLVO theory, a FORTRAN program was compiled to process the raw data of AFM spectroscopy. With the platform of the FORTRAN code, supporting calculations like integration, curve fitting and geometry analysis can be applied in the model, which will improve the calculation accuracy and reduce calculation speed.

The model also incorporates hydrophobicity of yeast and bacteria. Reaction equilibrium and Gaussian distribution theories are coupled in the model to achieve the analysis of solvent partitioning results for these cells. Statistical analysis and comparison of modelled data from the force curve calculation model was also supported by calculations based on Gaussian distribution and the Mann-Whitney $U$ test (MWW).

The mathematical argument for the analysis of AFM force curves is presented in the following chapter along with the compiled FORTRAN code for the zeta potential calculation and adhesion force analysis that will also be discussed and validated. The calculations built on an EXCEL platform for hydrophobicity and statistical analysis, are also discussed to explain how these models integrate with this study. 


\subsection{Force Curve Analysis Model with FORTRAN Code and Calibration}

\subsubsection{Import and Read of Deflection-Distance Curve}

The force spectroscopy measured data are exported and saved in the form of text files, which lists data as a deflection, in terms of electrical units ( $\mathrm{mA}$ or $\mathrm{mV})$, against a corresponding extension of the piezo electric scanner in units of distance (nm or $\mu \mathrm{m})$. The exported file will save the data in a particular format for different AFM systems. In this study, JPK Nano-Wizard II AFM and Park XE-100 AFM were used, the format of data saving for both systems is different. Thus, the first thing that the FORTRAN code undertakes is to sort the data appropriately so that it is in the right format for the subsequent processing and analysis.

\subsubsection{Transformation of Deflection-Distance Curve to Force-Distance Curve}

The raw data of deflection-distance Curves are then transformed to force-distance curves prior to calculation of electrostatic and adhesion forces.

The deflection- distance curve can be plotted as Figure.3.1. A slope can be observed in the contact region of the force curve.

According to Hooke's law, the cantilever bending is linear in relation to force change, which means that the deflection signal changes in a linear relationship to the cantilever bending. The slope is referred to as the slope of constant compliance, as for every incremental extension or retraction of the piezo-scanner, during the force measurement, there is a corresponding deflection of the cantilever as detected by the position sensitive photodiode. Thus, the inverse of the slope of this contact compliance region is referred to as the sensitivity coefficient and can be used to transform the raw data from a deflection-distance curve to a (deflection) distance-distance curve, as shown in Figure.3.1 B. 

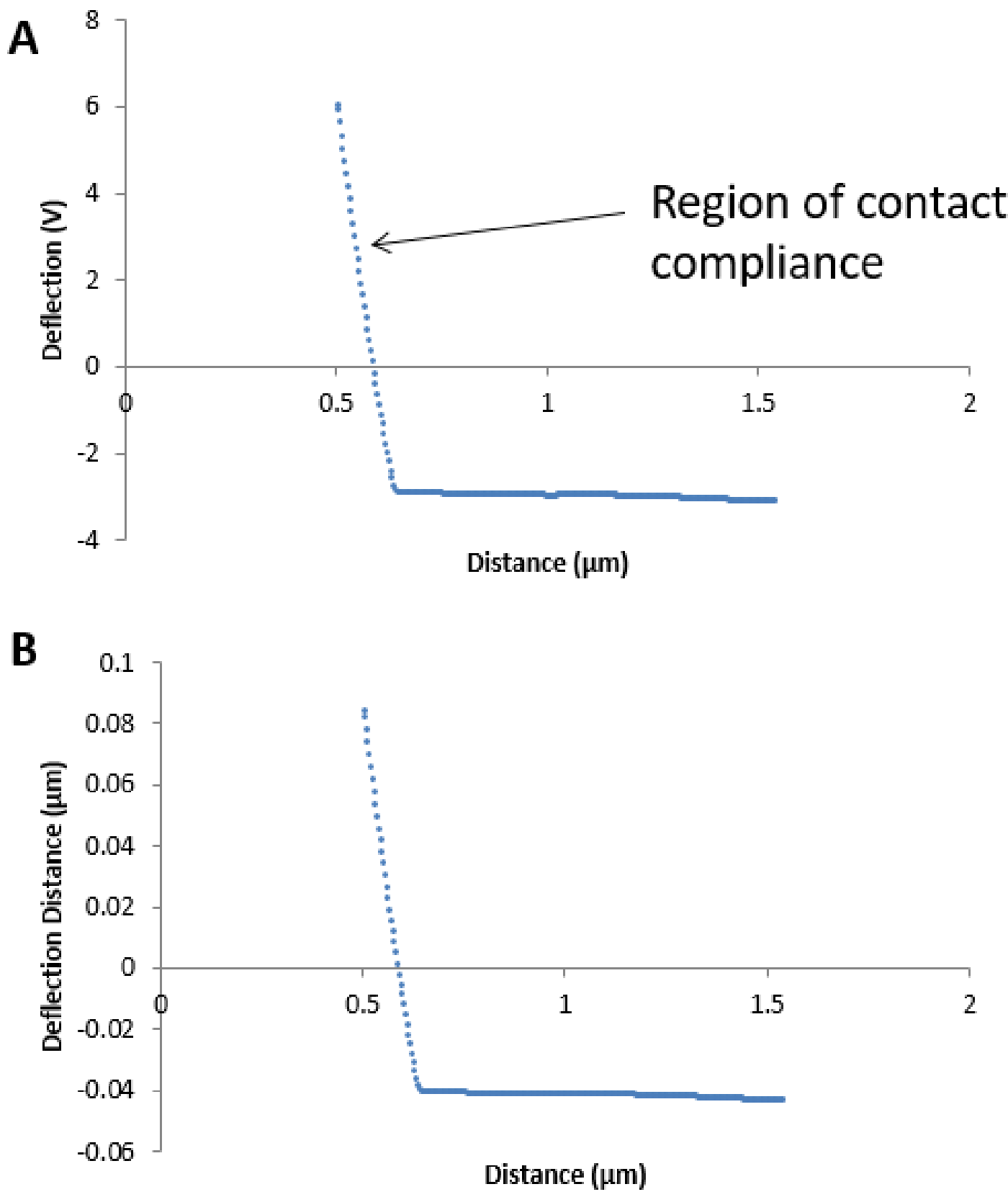

Figure.3. 1. Raw deflection-distance curve imported for calculation (A) and transferred deflection distance-distance curve (B).

The (deflection) distance-distance curve is then transformed to a force-distance curve using the cantilever's spring constant in Hooke's law. Thus, the deflection distance is multiplied by the with cantilever's spring constant and normalised by dividing the value by the diameter of the colloid probe $(\mathrm{N} / \mathrm{m})$. The next step in the transformation of the force curves is to account for the deflection of the lever in the 
separation distance between the colloid probe and the surface, this achieved by subtracting the incremental deflection of the lever from incremental movement of the piezo-scanner. Notice how the shape of the curve has changed from Figure.3.1 to 3.2. The force curve can now be plotted as a force against the separation distance, as shown in Figure.3.2 A, rather than the piezo-extension distance.

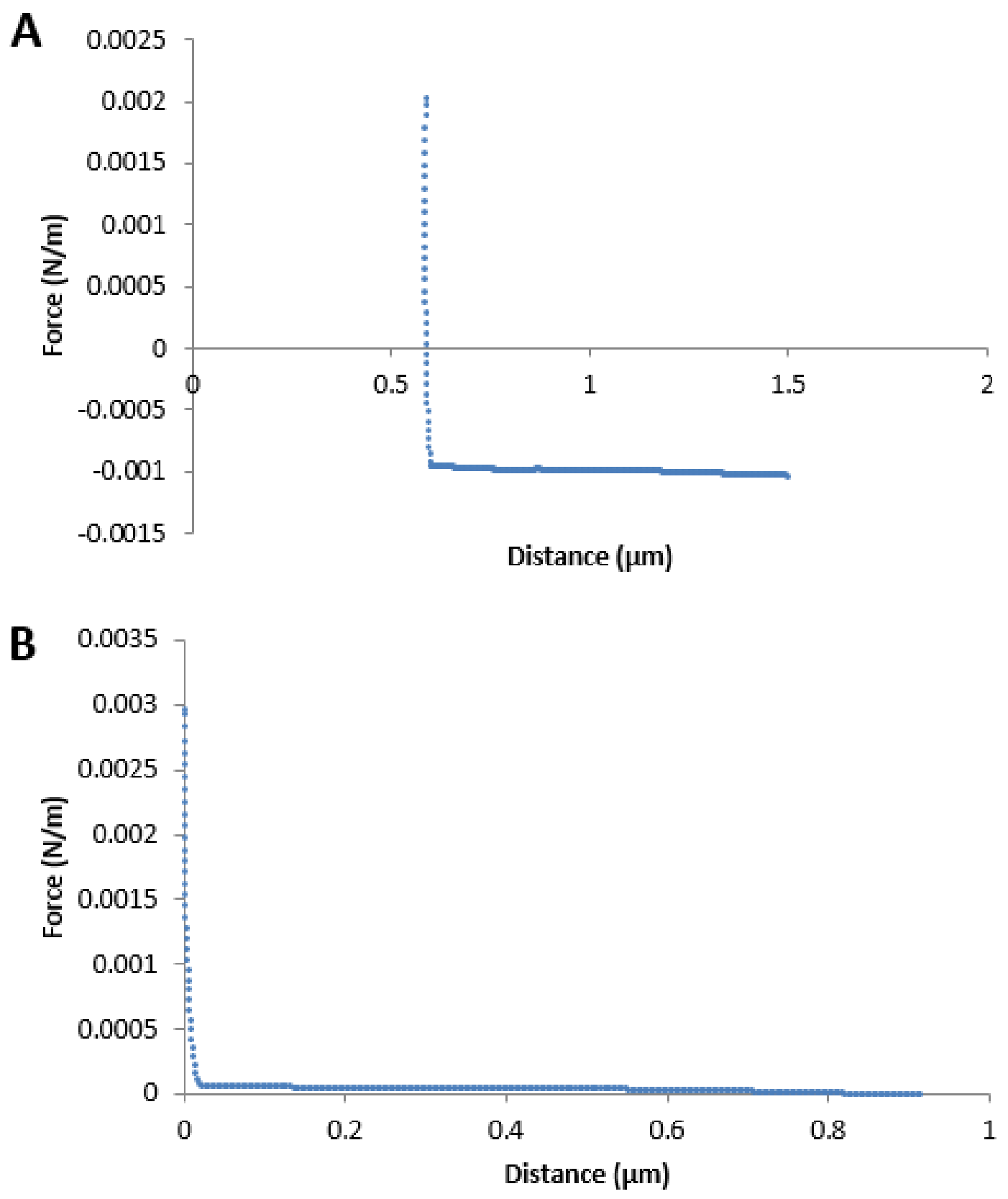

Figure.3. 2. Direct calculated force-distance curve from deflection distance-distance curve (A) and finial force-distance for following calculation (B). 
The arbitrary positioning of the laser beam on the position photodiode during laser alignment means that the zero distance must be identified when considering true separation distance. Zero-distance is normally defined at the start of the constant compliance within the approach curve but after removing the deflection from the piezo-ceramic distance to calculation separation there is a clear indication of the zero-distance position when the curve becomes vertical. Thus, a value can be subtracted from each separation distance to move the curve to the correct zero position (Figure.3.2B).

\subsubsection{Zeta Potential Calculation}

The zeta potential can be directly calculated from approaching force-distance curve. Zeta potential is only significant when close to the charged surface, so the calculation starts at defining the surfaces contact point on force curve. Zeta potential is an electrostatics energy that shows the energy of electrical field per coulomb. Therefore, theories (like the DMT) are applied to transfer force to energy to simplify the calculation. Finally, the zeta potential is calculated with Possion-Boltzmann equation (PBE) based on the DLVO theory together with the geometry of probe-surface contact.

\subsubsection{Definition of Points for Calculation}

In an AFM force curves, the force applied on a cantilever is constantly recorded. Electrostatic forces contribute to in the force curve but are present alongside other interactions so that the force curve is a convolution of the forces. However, DLVO theory allows deconvolution of the force curve so that electrostatic force can be

calculated. The calculation was achieved by several previous studies in simulation of intermolecular interactions (Bowen et al., 2002; Brant \& Childress, 2002; Ruiz-Cabello et al., 2014). The present study is different from this previous research, in that the calculation is based on a measured force curve. A new problem of this 
study is contact point definition, and the provision of an accurate position where the influence of the zeta potential on force curve is most significant.

Based on the Boltzmann equation, electrostatic force will increase in an exponential relationship when the probe approaches the surface until contact. Its influence will become significant at short probe-surface separation distances.

The shape of AFM force curve, Figure.3.3, shows force increases with a large gradient after contact while force increase before contact is negligible. The force gradient changes a lot at the contact point. The average gradient $(\mathrm{G})$ of the whole force curve can be calculated with the starting point (farthest distance) and ending point (largest force). The average gradient is obviously larger than the gradient before contact and smaller than the gradient after contact, as shown in Figure.3.3. The assumed mean gradient $(\mathrm{G})$ is used as a critical gradient to define the contact point because of its significant difference and relationship to the contact and non-contact force change.

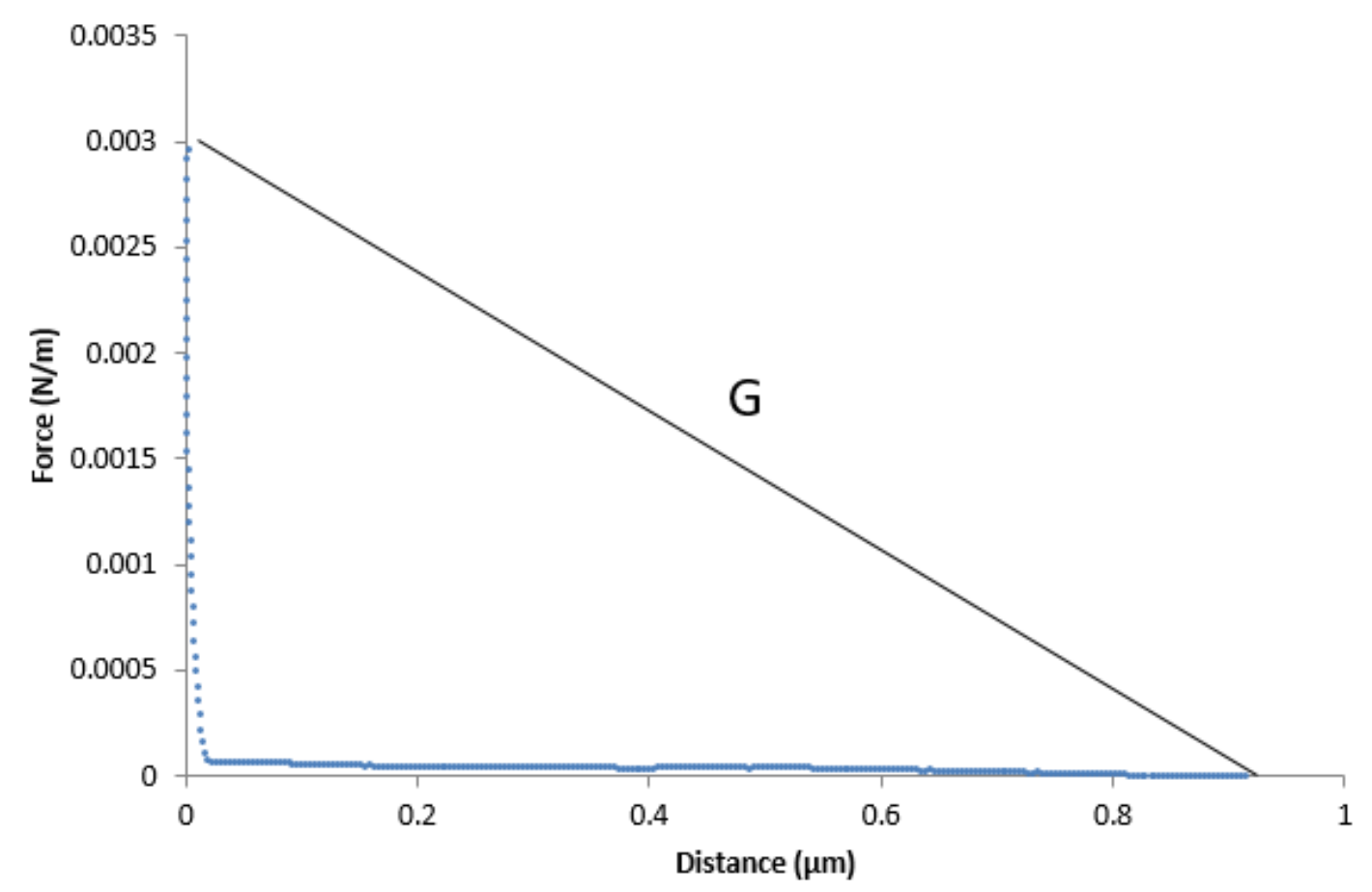

Figure.3. 3. AFM force curve with the mean gradient $(\mathrm{G})$ of whole force curve force change assumed in gradient comparison.

Gradient calculation $\left(\mathrm{g}_{\mathrm{i}}\right)$ is applied between two neighbouring points on the force 
curve from the starting point, gradient $\left(\mathrm{g}_{\mathrm{i}}\right)$ was calculated and compared with the value $\mathrm{G}$. If $\mathrm{g}_{\mathrm{i}}$ is smaller than $\mathrm{G}$, it infers that the points are before contact. Otherwise, a $g_{i}$ larger than $\mathrm{G}$ means the points are in the region after contact on the force curve. The first $g_{i}$ that is larger than $G$ identifies the contact position, and the value $i$ is recognized as the point of contact on the force curve. The FORTRAN program is set to detect the points from the raw data in a routine until find the value $i$. The number $i$ and i-1 (last point before contact) is then used in force and distance sequences to extract the force and distance data at and just before probe-surface contact.

The gradient check is easily obstructed by noise in AFM force spectroscopy, especially when distance between two points is small. Curve fitting is necessary when distance between two recorded points are small. Applied in the FORTRAN program, it can be expressed as Equation.3.1 for both distance and force.

$P F_{i}=\frac{\left(P_{(i(n-1)+1)}+P_{(i(n-1)+2)}+\cdots P_{i n}\right)}{n}$

Equation.3.1

Where $\mathrm{PF}_{\mathrm{i}}$ is fitted point, $\mathrm{P}_{\mathrm{i}}$ is the raw data point and $\mathrm{n}$ is the number of raw data points for average calculation. The fitted points that are used in the gradient analysis for zero distance identification can significantly reduce obstruction by noise.

\subsubsection{Energy Calculation from AFM Force Curves}

The membrane surface and silica colloid are both charged surfaces. AFM analysis of the membrane surface with a silica colloid probe is undertaken in a conductive solution. Thus, DLVO theory can be applied for the analysis of surface electrostatic interactions between the two surfaces (Hunter, 1989). As the electrostatic potential that is applied to the surrounding environment of a surface, zeta potential can be regarded as the surface potential in calculation and its decrease with distance from a flat charged surface can be derived from Equation.3.2 (Verwey \& Overbeek, 1948). The total energy can be calculated as Equation.3.3.

$$
\begin{array}{ll}
\Psi_{C}=\Psi_{o} \exp (-\kappa h) & \text { Equation.3.2 } \\
E=\Psi_{z} \mathrm{C} & \text { Equation.3.3 }
\end{array}
$$


Where $\Psi_{\mathrm{c}}$ is local potential, $\Psi_{\mathrm{o}}$ is surface potential, $\mathrm{h}$ is distance to surface, $\kappa$ is Debye constant, $\Psi_{\mathrm{z}}$ is electrostatic potential C is overall electric charge on the surface in coulomb and $\mathrm{E}$ is total energy. Total energy (E) can be calculated from the force detected by AFM force spectroscopy by DMT and JKR theories, which describe the non-adhesive energy in colloidal contact as Equation.3.4 (Grierson, Flater, \& Carpick, 2005).

$$
F=2 \pi E R
$$

Equation.3.4

Where $\mathrm{F}$ is force, $\mathrm{R}$ is the radius of particle. The overall charge can be calculated with surface charge and area of charged surface, because surface charge is the density of charge applied on the surface. It can be described as Equation.3.5.

$$
C=A \delta
$$

Equation.3.5

Where $\mathrm{A}$ is the charged surface area and $\delta$ is surface charge. Combining equation.3.3 - 3,5, the mathematical relationship between surface potential and its related colloid properties can be straightforwardly expressed as Equation.3.6 for flat surfaces in contact (constant separation distance across the interaction area). The separation distance between the silica colloid and membrane surface is not constant at different positions across the colloid surface. Thus, the geometry influence is significant in the probe-surface electrostatic interactions. The zeta potential calculation should be an integration across the probe surface that can be defined by Equation.3.7.

$$
\begin{aligned}
& \Psi_{c}=\frac{F}{2 \pi R} \frac{1}{A \delta} \\
& \int_{0}^{R} \Psi_{c} d r=\int_{0}^{H} \frac{F}{2 \pi R} \frac{1}{A \delta} d h \\
& n=n_{\infty} \exp \left(\frac{-e v \Psi}{K T}\right)
\end{aligned}
$$

Equation.3.8

Where $\mathrm{n}$ is the number of ions on the surface, $\mathrm{n}_{\infty}$ is the number of ions far from the surface, e is electron charge, $\mathrm{v}$ is the valency of ion, $\Psi$ is the potential, $\mathrm{K}$ is Boltzmann constant and $\mathrm{T}$ is temperature in $\mathrm{K}$. Calculation of the total energy in this study is based on Equation.3.7. There are many phenomena that can cause the electrostatic potential to become less significant inside the Debye length, for example, charged ions adsorbed on the membrane surface (Calvo et al., 1996), dense layers 
attracted by the charged surface (as shown in Equation.3.8), no effective diffusion inside outer Helmholtz plane (OHP) and Van der Waals forces (Verwey \& Overbeek, 1948).

\subsubsection{Geometric of Spherical Surface Contact with Flat Surface}

The geometry of a probe-surface contact means that the separation distance between the two surfaces is significantly different at different positions. The field potential at a location on the spherical surface is influenced by separation distance from the charged membrane surface. The influence is described mathematically as Equation.3.2.

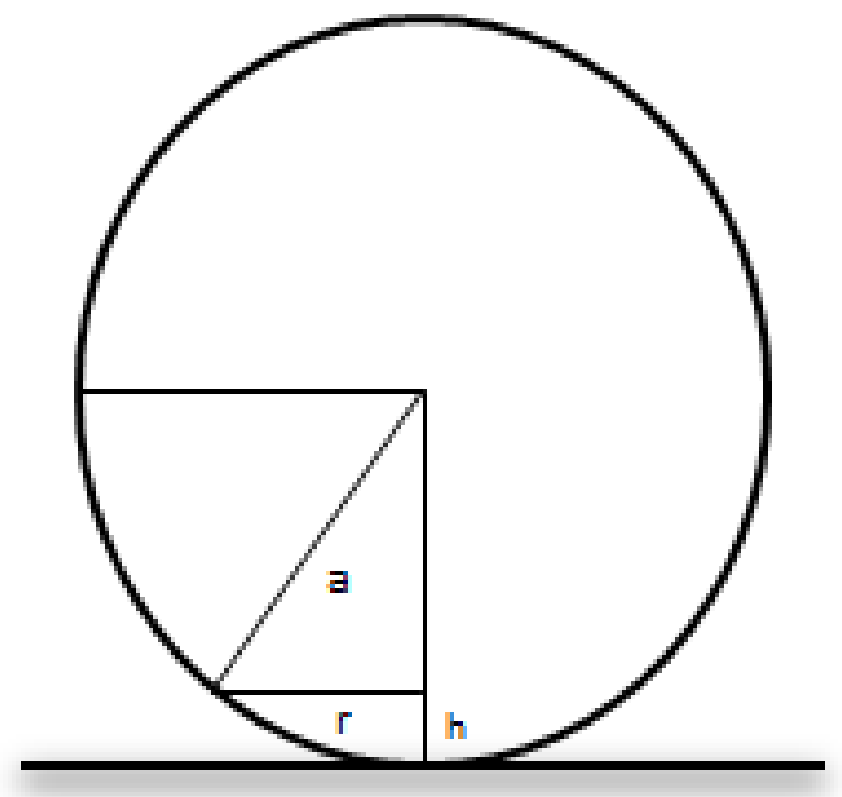

Figure.3. 4. Geometry of the silica colloid probe and membrane surface, illustration of the relationship between bead radius (a), cross section radius (r) and local height away from membrane surface $(h)$.

The surface of colloid probe can be regard as a sphere. The effective area of probe surface is in fact the bottom hemisphere of the particle, as show in Figure.3.4. The total area of the spherical cap under a height can be geometrically presented as Equation.3.9 (Polyanin \& Manzhirov, 2006). With the assumption of an ideal 
spherical silica bead, the surface area at the same height can be calculated from Equation.3.10. Mathematically, the height difference $\Delta \mathrm{h}$ is infinitely close to zero as a differentiation term.

$$
\begin{array}{ll}
A=2 \pi a h & \text { Equation.3.9 } \\
A=2 \pi a \Delta h & \text { Equation.3.10 } \\
r=\sqrt{a^{2}-(a-h)^{2}} & \text { Equation.3.11 }
\end{array}
$$

Where A is surface area, a is radius of sphere, $h$ is height of the cap and $r$ is the radius of cross-section of the cap. The cross-section radius can be expressed with particle radius and height from membrane surface with Pythagorean theory, as shown in Equation.3.11. The calculation of interaction area (A) and separation distance (h) that is used in zeta potential calculation can be described as Equation.3.10 and 11. Applied with the zeta potential integration (Equation.3.7), the influences of differential calculation (Equation.3.10) at different positions can be integrated with the result of the overall influence of the electrostatic force on the probe-surface interaction.

\subsubsection{Integration across the Spherical Surface Geometry}

The integration that was employed in the zeta potential calculation (Equation.3.7) contained two independent variables of height and cross-section radius, two dependent variables of interaction area and electric potential at location. Cross-section radius can be calculated from Equation.3.11, which means the variable $\mathrm{r}$ is related to $\mathrm{h}$. The integration with independent variable of $r$ can be replaced with $h$ if the logic of Equation.3.11 is applied. Equation.3.7 can be rewritten to Equation.3.12.

$$
\begin{aligned}
& F=\int_{h_{o}}^{H} 2 \pi R A \delta \Psi_{o} \exp (-k h) d h \\
& A_{c s}=\pi\left(\frac{D}{2}\right)^{2}=\pi a^{2}
\end{aligned}
$$

Where $\Psi_{0}$ is the average of the zeta potential at a small area of the membrane and $\mathrm{D}$ is diameter of the spherical particle. The area used in average zeta potential 
estimation is as show in Figure.3.5. It is the area underneath the colloid probe, which geometrically equal to cross-section area of probe and calculate from Equation.3.13.

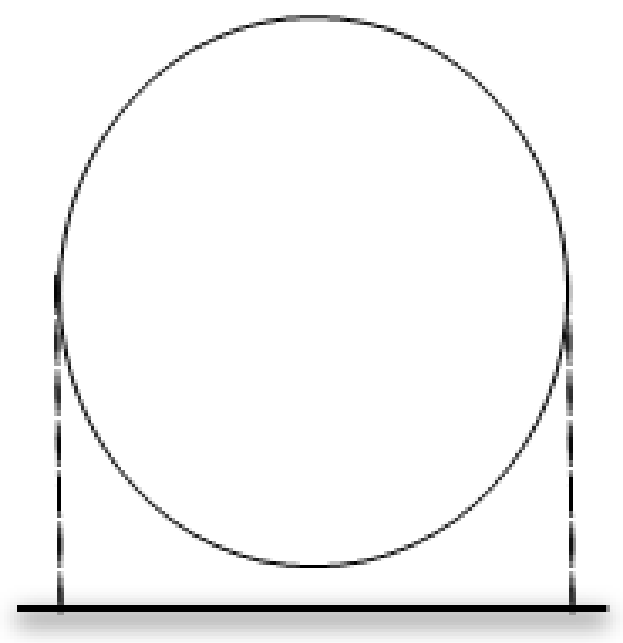

Figure.3. 5. Area of the membrane sample engaged in zeta potential calculation.

The diameter of the testing area is known from the size measurement of the colloid probe. The whole calculation is under the assumption of constant zeta potential within the interaction area. The two dependent variables of colloid probe bottom cup area and zeta potential at location have a complicated mathematical performance after being integrated. Thus, basic theories of integration (Johnson, 2012) were applied to simplify the integration by segmenting the calculation on the bead surface into small steps as shown in Figure.3.6.

A few thousand segmentation stages are required to guarantee the accuracy of integration, and the influence of the number of stages are discussed in the verification section of this chapter (section.3.2.6.1). An approximation of the average height on each stage is demonstrated in Figure.3.7. The average level of each stage is calculated with the probe geometric and mean value method as described in Equation.3.14. Each stage will be narrow ring shape and approximately at the same height as the mean value. Stage area can be calculated with Equation.3.15. The separation distance of the stage to the membrane surface is the sum of the distance of the bead bottom to the membrane and vertical level difference from mean stage level to bead bottom, as 
show in Figure.3.7.

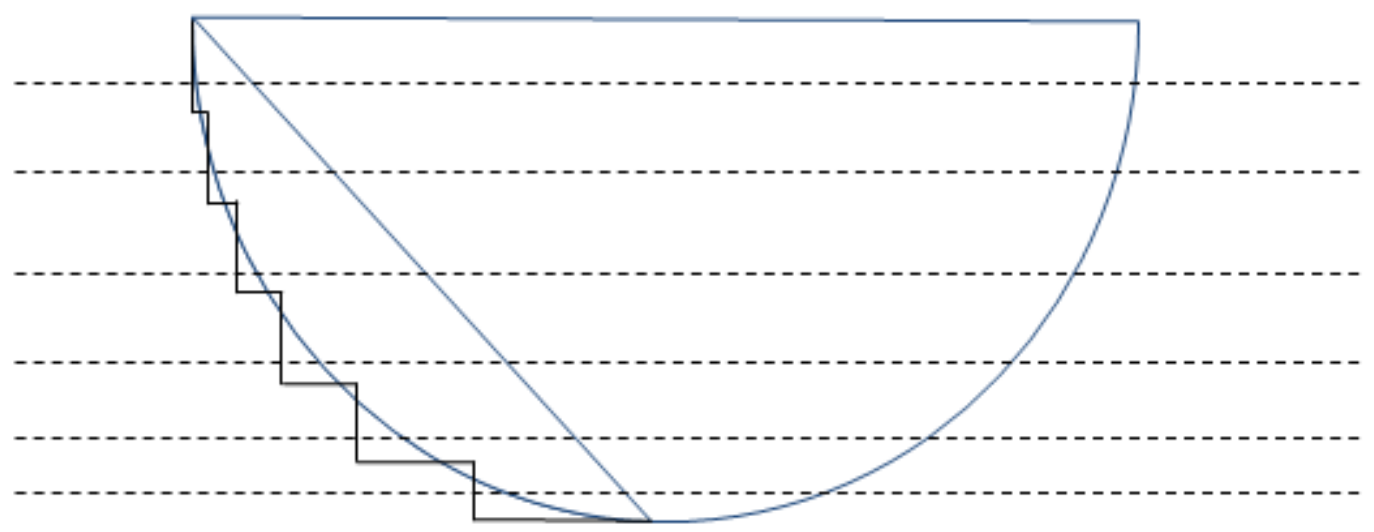

Figure.3. 6. Spherical surface segmented into 6 stages for calculation. Dashed lines show the mean height of the stages.

$$
h_{\text {mean }-n}=\frac{\left(h_{n}-h_{n-1}\right)}{\ln \left(\frac{h_{n}}{h_{n-1}}\right)}=\frac{\sqrt{a^{2}-r_{n-1}^{2}-\sqrt{a^{2}-r_{n}^{2}}}}{\ln \left(\frac{a-\sqrt{a^{2}-r_{n}^{2}}}{a-\sqrt{a^{2}-r_{n-1}^{2}}}\right)}
$$

$A_{n}=\pi\left(r_{n}^{2}-r_{n-1}^{2}\right)$

Equation.3.15

Where $\mathrm{r}$ is the radius of each stage. Illustrated in Equation.3.12, the electric potential is related to the exponential of local distance to the surface. The two dependent variables of probe surface area and electric potential can both be replaced with expressions as in Equations 3.14 \& 3.15.

Therefore, zeta potential is estimated by integration at the different stages. The approximation of zeta potential influence at the specified area on the colloid probe for each stage is calculated with Equation.3.12, 14 and 15. Approximate potential can be calculated with the measured force from the JKR model, as shown in Equation.3.4. Total potential can be converted to zeta potential from the calculation of Equation.3.3. 


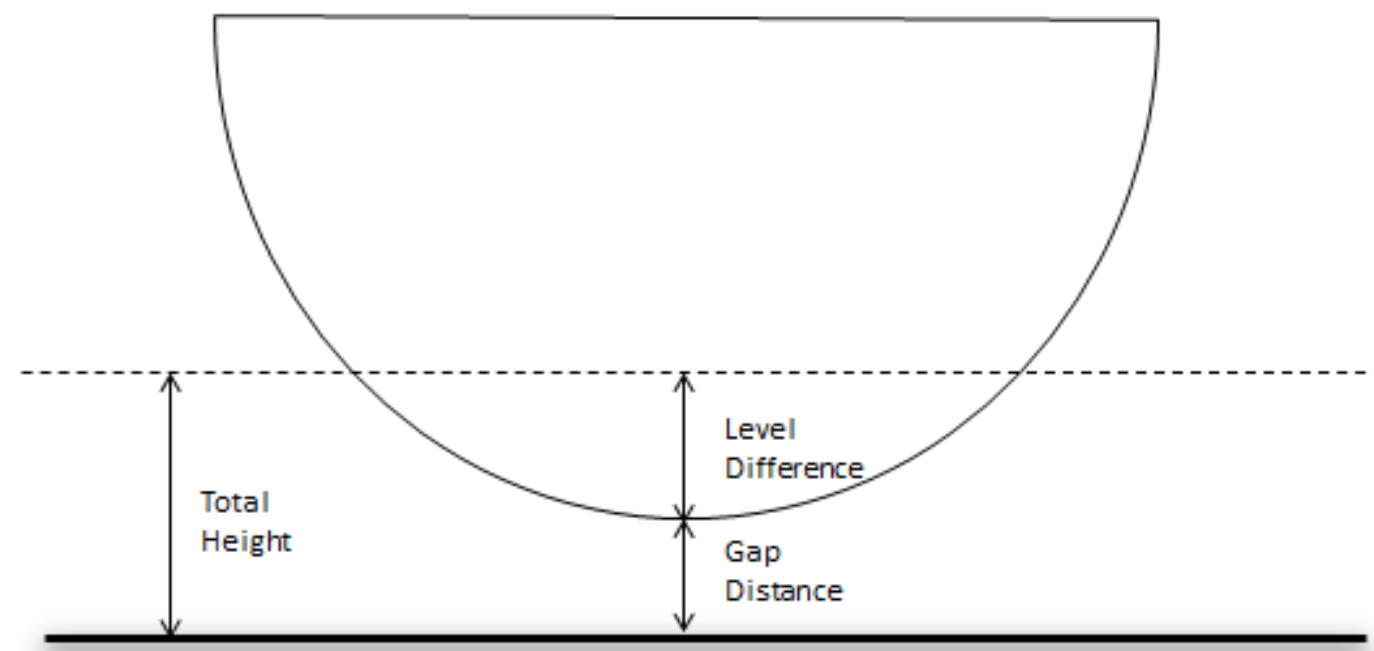

Figure.3. 7. The composition of total height for each stage to membrane surface. Two parts of the total height include level difference to bottom and bottom to membrane distance.

\subsubsection{The influence of Membrane Surface Deformation on the Zeta Potential Measurement from AFM Force Curve}

The membrane surface is regarded as an ideal flat surface in the computation to simplify zeta potential calculation in reviewed theories, as stated in section.1.2.1. In reality the surface rough, for both DK and Cyclopore membranes, as shown by AFM contact mode imaging in Figure.3.8 c \& d. The influence of surface curvature and geometry of interaction is apparent in Figure.3.8 a \& b, where the image is made using a scanning colloid probe. The FORTRAN program developed assumes that the surface is ideally flat on the level of contact. The force measurement will be influenced by the surface deformation as well, even if a relative flat surface was selected in measurement process and the extent of the double layer may smoothen the contact surface. The Curvature of membranes can be measured with line analysis on AFM contact mode imaging, as shown in Figure.3.9. 

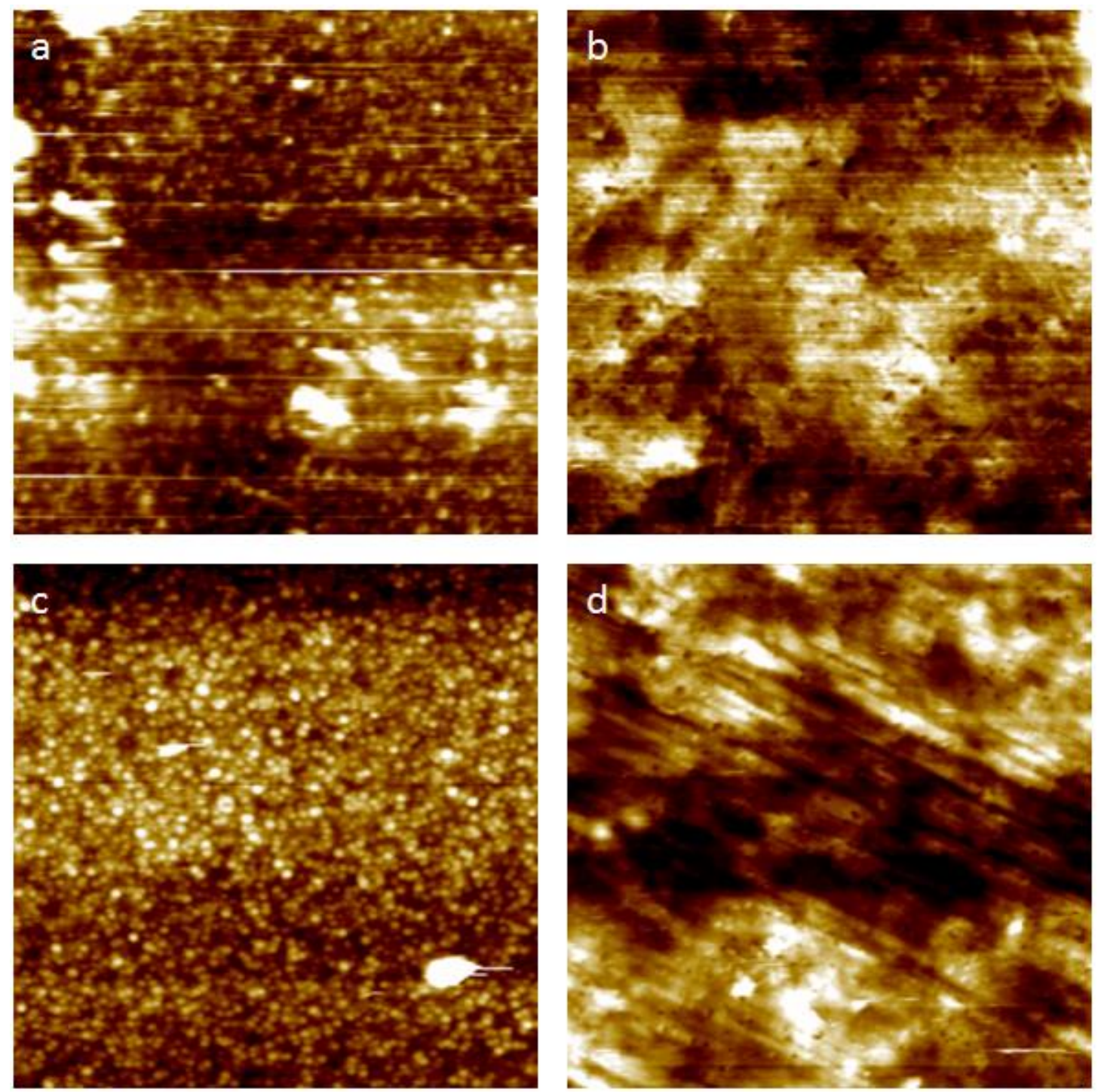

Figure.3. 8. Contact-model scans of (a) DK membrane surface and (b) Cyclopore membrane surface topography with colloid probe of $33.5 \mu \mathrm{m}$. Imaging of membrane surface with contact tip (c) DK membrane and (d) Cyclopore. All images are in size of $9.47 \mu \mathrm{m}$ X $9.47 \mu \mathrm{m}$.

The theories used in FORTRAN zeta potential computation do not consider surface deformation. Figure.3.10 is the illustration of difference between the mean calculation (assumption in DLVO theory) and reality when the colloid probe contacts the surface. There is a height difference between the real local heights from a random point on the bead surface to the mean estimation height to the flat surface. As show in Figure.3.10, $a$ is the mean surface in calculation and the real surface is present as $b$. There is a significant difference $\left(\mathrm{H}_{\text {diff }}\right)$ between the separation distance calculated for 
the mean surface and the real surface.
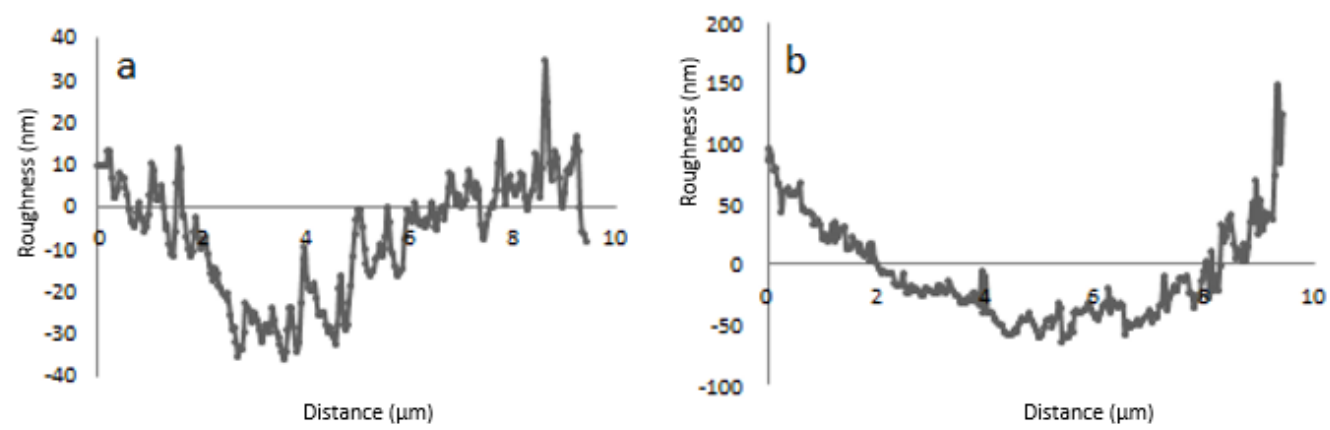

Figure.3. 9. Line analysis of (a) DK membrane and (b) Cyclopore membrane surface, $\mathrm{Y}$ axis is in $\mathrm{nm}$ and $\mathrm{X}$ axis is in $\mu \mathrm{m}$ for both $\mathrm{a}$ and $\mathrm{b}$.

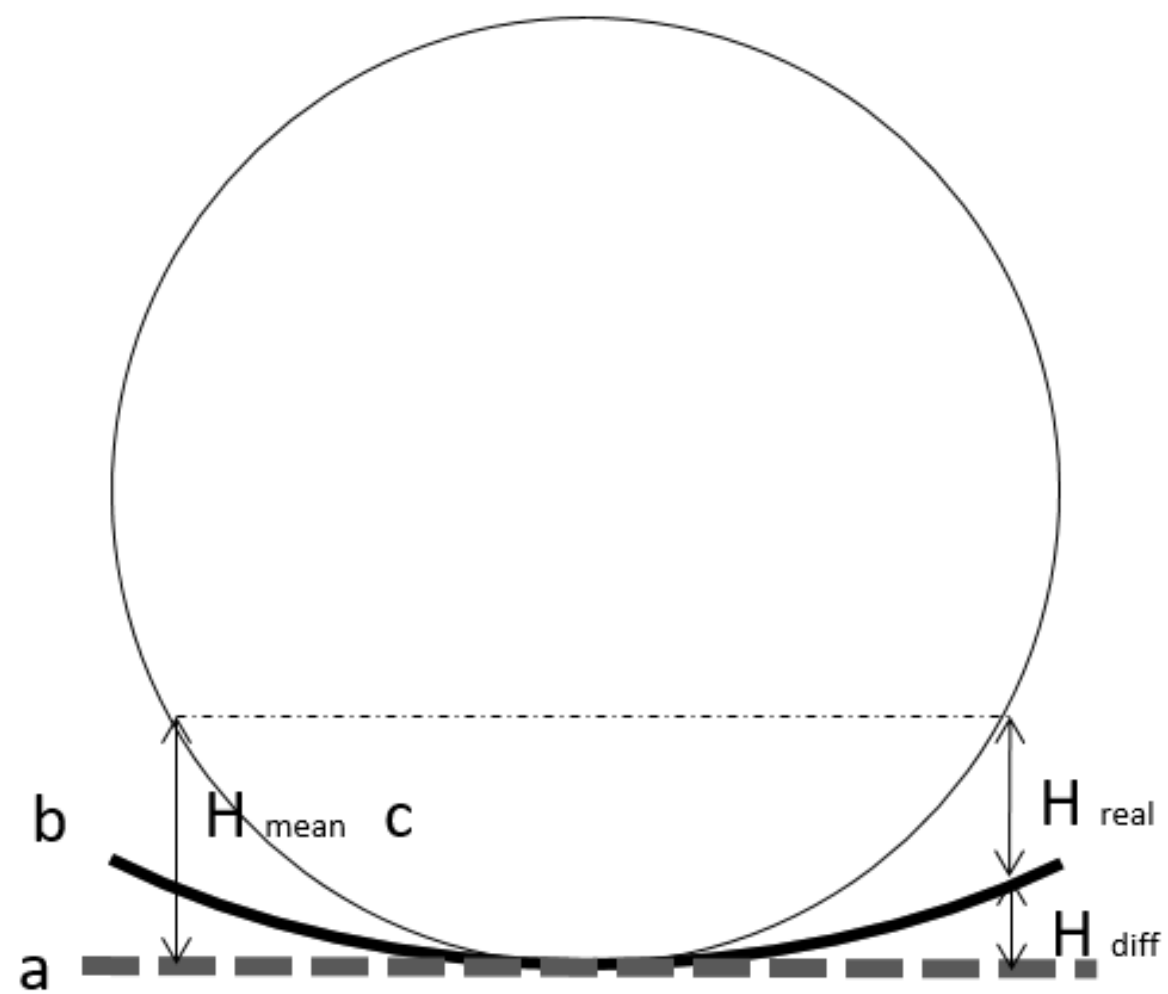

Figure.3. 10. The reality of contact surface. $a$ is the bead surface, $b$ is the real contact surface of membrane, a is the mean flat surface used for FORTRAN calculation. $\mathrm{H}_{\text {mean }}$ is the height used in ideal computation. $\mathrm{H}_{\text {real }}$ is the real distance between two surfaces.

$$
a=\frac{h^{2}+r^{2}}{2 h}
$$

Incorporation in the calculation of the surface effect is similar to the calculation of silica bead surface geometry effects on zeta potential calculation. The surface is 
approximated as a small fraction of a sphere with a cross-section radius (r) and bending height $(\mathrm{H})$, shown in Figure.3.11, can be measured with AFM imaging and used in the FORTRAN program for each force curve analysis. The radius of the assumed sphere (a) can be derived with Pythagorean theory, to finally derive Equation.3.16. Therefore, the influence of surface curving on gap distance between the probe and sample surface can be estimated with a geometric calculation. However, this will be more complex in the influence on zeta potential because of the non-linear potential attenuation increases with distance.

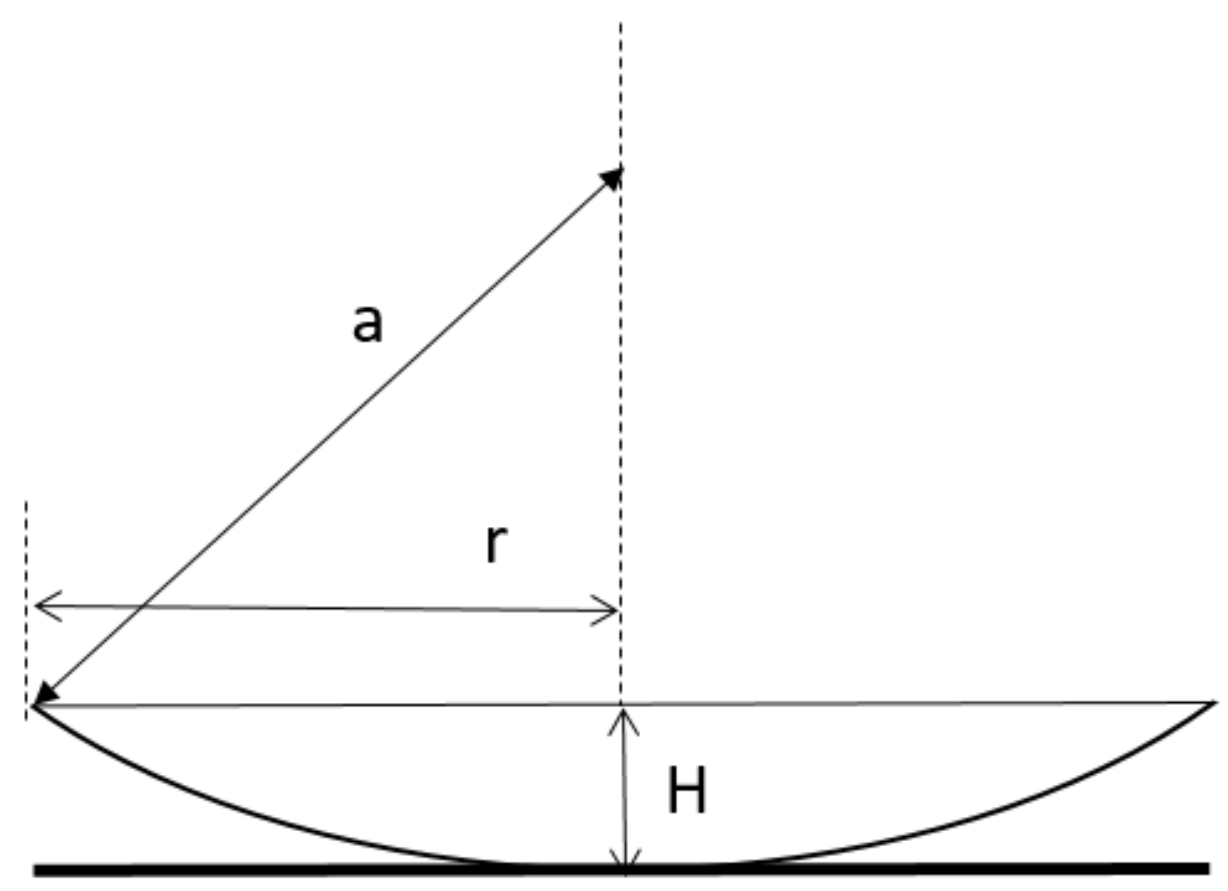

Figure.3. 11. Approximate assumption of the spherical geometry on the membrane surface bending. The parameter of cross-section radius ( $\mathrm{r}$ ) and bending height $(\mathrm{h})$ are measured through AFM imaging.

The influence of surface curving on zeta potential estimation from AFM force curves can be calculated as a ratio. The ratio is derived from Equation.3.12 and shown as Equation.3.17. The real distance between the two surfaces is defined in Equation.3.18. The ratio can be calculated from Equation.3.19 with both integrations calculated from a mean flat and approximate reality of the membrane surface.

$$
\text { integration }=\int_{0}^{r} \mathrm{~A} * e^{(-k h)} d h
$$




$$
\begin{aligned}
& H_{\text {real }}=H_{\text {mean }}-H_{\text {diff }} \\
& \text { ratio }=\frac{\text { integration }_{\text {real }}}{\text { integration }_{\text {mean }}} \\
& \Psi_{\text {real }}=\text { ratio } \times \Psi_{\text {mean }}
\end{aligned}
$$

The ratio can be simply applied on the mean zeta potential calculate to optimize the mean flat membrane surface assumption. Equation.3.20 was used to transfer the mean zeta potential calculation from the flat surface to the zeta potential that is applied on a curved surface. The calculation structure is not significantly influenced by the optimization if applied as Equation.3.20.

\subsubsection{Adhesion Analysis from Force Curves}

In some of the force curves measured, adhesion forces can be observed directly from the retraction (dispatchment) force curves. Review of previous AFM force measurement research on biological surfaces also shows it is possible to have an adhesion between colloid probes and sample surfaces (Puech et al., 2005), as described in section.1.3.3. Most of these previous investigations only analysed the maximum adhesion forces. However, from the comparison of their presented force curves, there is more information concealed in the adhesion force curves that causes difference in force curves from different research (Marie et al., 2013; McNamee et al., 2006). Adhesion analysis is based on mathematical methods that were applied in the FORTRAN program.

\subsubsection{Maximum Adhesion Analysis}

Maximum adhesion force is an observable force in the retraction force curves. In the analysis of a retraction force curve, maximum adhesion can be observed directly. Numerical tools, as described below, are used in the FORTRAN program to improve the adhesion force measurement accuracy from examining data points in retraction force curves. 
The adhesion force is an opposite direction force to the cantilever bending force, which is normally marked as a negative force. A minimum determination, written as a 'find minimum routine' in FORTRAN, allowed the simple identification of the maximum adhesion. However, in some force curves with small adhesion or even no adhesion, the calculation method will still suggest a minimum force as the maximum adhesion force. A comparison is applied to determine if the maximum adhesion is significant. The calculated adhesion force is compared with maximum load on cantilever, which can be calculated from a find maximum routine. 5\% was selected as the critical value of significance. Thus, the found minimum is determined as maximum adhesion if it is an absolute value larger than $5 \%$ of the maximum load, otherwise no significant adhesion was recorded; this criterion was set with the standard maximum load of the present study of $1-2 \mathrm{nN}$. This criterion was reached after manual checking of the process at lower and higher thresholds. At higher thresholds adhesion events were missed, at lower thresholds the calculation collapsed.

\subsubsection{Adhesion Components Analysis}

In some of the adhesion force curves, there was more than one kind of adhesion component present. With different types of bonding, these adhesion components will have different strength and break at different levels when retracing from surface. The trend of the retraction force curve will be changed at points where the adhesion is applied or broken. The change at these points influence the gradient of force curves. Gradients will become negative when adhesion force increases and it will turn to positive when adhesion breaks and force is released from the cantilever. Numerical methods that are based on gradient calculation and comparison can find out these changes. Therefore, an adhesion component calculation routine is applied within the FORTRAN program to achieve the goal of adhesion components analysis.

The criteria of $5 \%$ of maximum force is applied to identify where the significant adhesion started and ended. (i.e. the cut off points are identified by the code when the 
adhesion becomes less than of the loading force.) The FORTRAN code focuses on the part of the force curve that contains all the significant adhesion events. Gradient calculation is applied. A series of gradient changes in the adhesion force curve are determined from the calculation.

If two adjacent gradients are in the same direction, it means there is no adhesion component break or application. Otherwise, different directions of adjacent gradients within a force curve adhesion component demonstrate an adhesion breaks or adhesion application sensed by the cantilever.

\subsubsection{Contact Position Error}

The AFM force curve consists of data points that are measured by the position sensitive photo diode as the sample and the probe are brought into contact and retracted. The number of points that are measured over an approach/ retraction distance can be selected within the AFM software. Figure.3.12b shows the distance between each point is less than $5 \mathrm{~nm}$, however, this is a significant gap compared to the Debye length. The gap was always as large as that shown in Figure.3.12b for force curves measured with PARK XE-100 AFM because of the set 512 point which the software permits. This interpoint distance will be smaller when JPK nano-wizard AFM was used, because more points were able to measure.

Contact is defined as the initiation of the electrical double-layer overlap. The contact point approximation is shown as point 1 in Figure. $3.12 \mathrm{~b}$ and as point 2 in Figure.3.12c. The Figure.3.12c shows negligible force increase before probe contact on the surface, point 3 . The force growth after contact is not as significant as that increasing with full contact (point 1 to 2 in Figure.3.12c). Therefore, there are two situations that may exist during the close surface approach used in zeta potential calculation. Contact happened on the detected contact point, Figure.3.13b. Otherwise, the contact occurred in the 'blind' area between the two points (Figure.3.13a). If this was the case, in the calculation, the program will regard the contact as on the higher 
data point. The FORTRAN zeta potential calculation program would then use the two points after 'contact' to calculate a force change, which was then used in the zeta potential calculation. However, in this case the calculated value of the zeta potential would always be extremely large and non-sensical, and so the data from this force curve was ignored in subsequent calculations. This scenario was not typical, most of the time the FORTRAN program calculated a zeta potential that was at a reasonable scale, thus indicating that the zero-distance had been optimally identified.

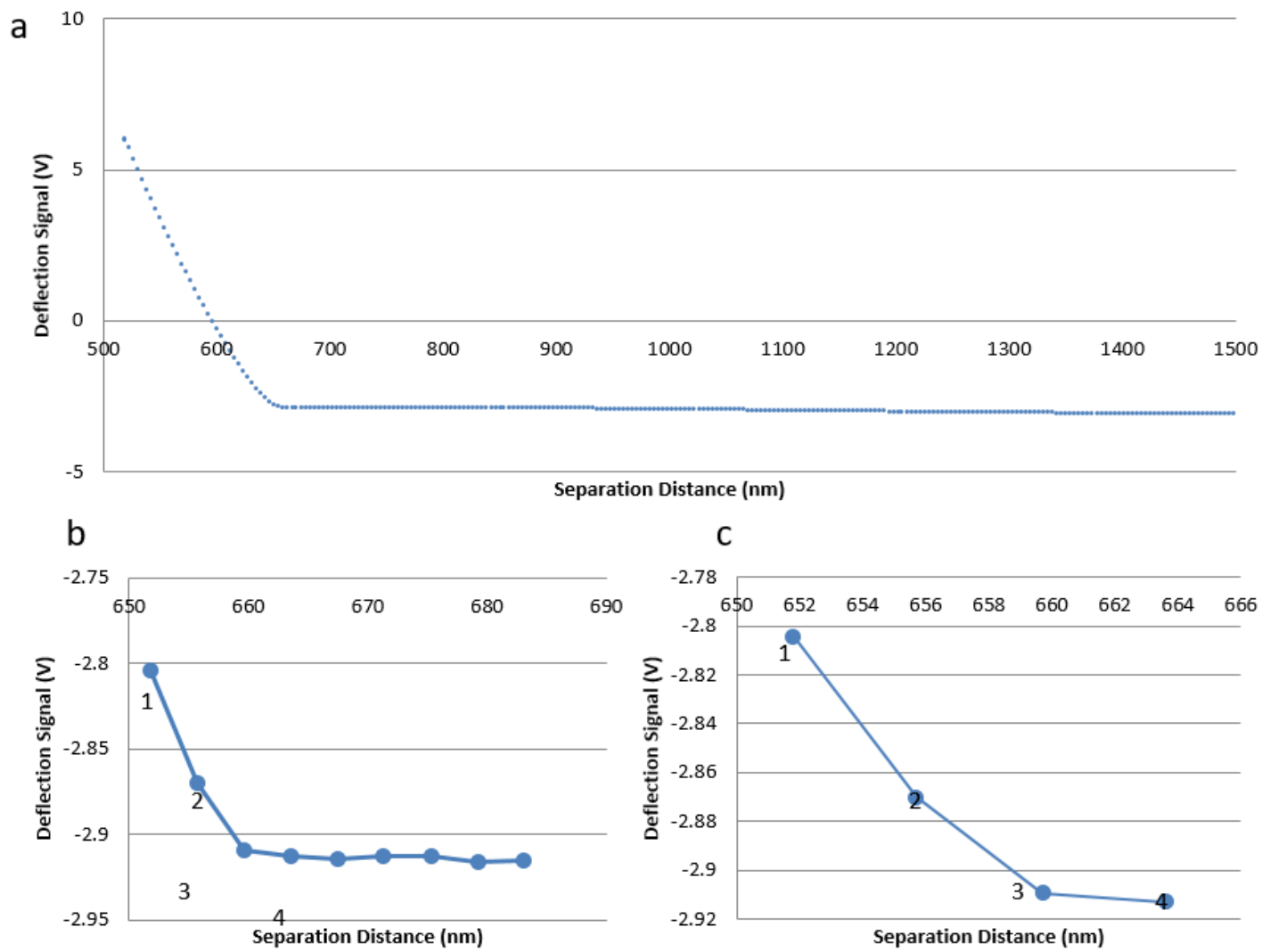

Figure.3. 12. $a$ is the overall force curve raw data detected by Park XE-100 AFM. $b$ is focus on the contact region on the raw force curve. Point 1, 2 and 3 are defined as point contact, 1 point and 2 points before contact. For c, contact point is as 2, point 3 as the point before contact and point 4 as two points before contact. Point 1 is the first point after contact position. 
a

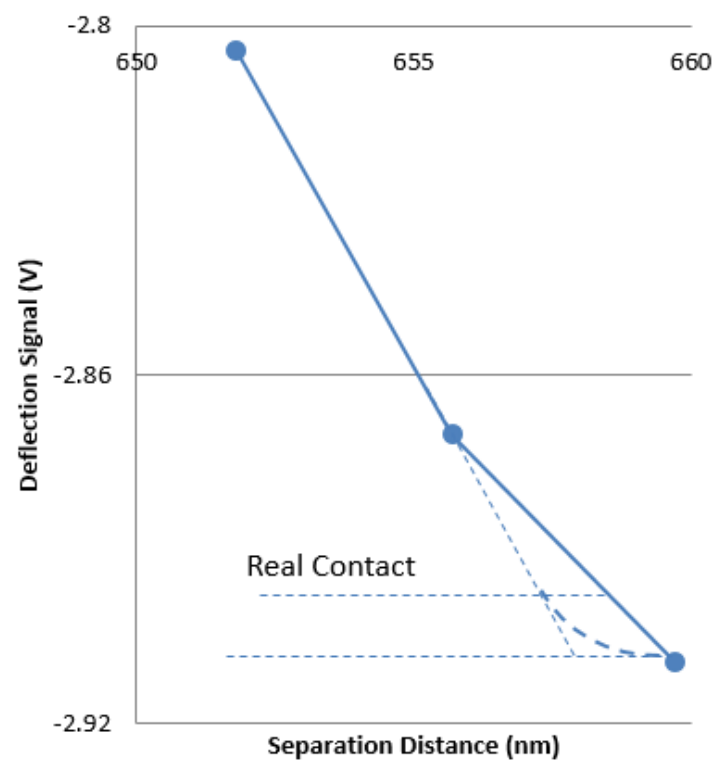

b

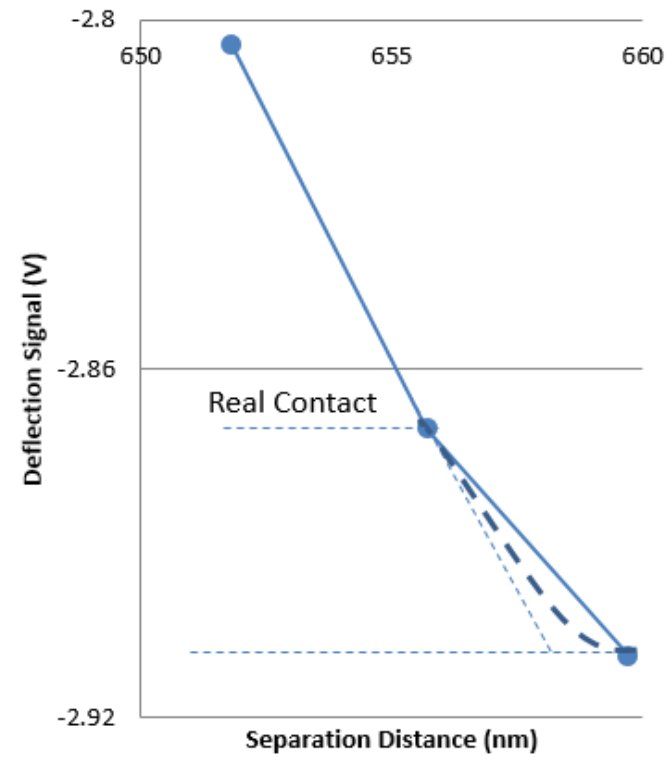

Figure.3. 13. $a$ is the real contact in between the two points involved in zeta potential calculation (contact point and one point before contact); $b$ is the real contact exactly on the contact point.

\subsubsection{Validation and Calibration of Force Calculation in the FORTRAN Code}

The whole calculation system is focused on electrostatic interaction and adhesion force. It is more complex for electrostatic force calculation. DLVO theories are applied in the calculation of zeta potential. Van der Waals forces are not considered in the calculation when using DLVO theory in the program. Because it is assumed to be a negligible force at the separation distance used in the calculation, and the assumption is verified in this section as that follows. The calculation of zeta potential will be validated by comparison to the work of previous researchers and their calculation to ensure the reliability and validation of the method presented in this thesis.

\subsubsection{Calibration of Surface Differential Calculation for Precision Control}

In the calculation of probe surface area contact, there is a differentiation across the bottom half of the colloid probe to calculate the area of the probe that interacts 
with the surface. The area must be verified to ensure the calculation base. In this calculation, the distance change at each step increases with height of the step (above the surface). This feature is a benefit for calculation precision as closer to the sample surface the incident forces will be larger. More accurate calculation at the stronger force part is guaranteed in the setting; more calculation steps at a smaller scale will achieve more accurate calculation.

$A=\pi r^{2}$

Equation.3.21

The area is calculated with the differentiation and equation of circle area, Equation.3.21, for comparison. The error induced in the calculation is plotted as percentage error against calculation steps, as shown in Figure.3.14. Calculation with 200 steps induces error of around 1\%, and error is decreases when using more calculation steps. In the calibration of the whole program, 1000 differential steps were used and found only to cost less than 1-minute calculation time (multiply calculation of a few dozen force curves). The error was found only to be $0.2 \%$ at 1000 steps. A much higher step number was applied when using the program $(5000-15000)$, which makes the error in interacting area reduced to a negligible level in all calculations.

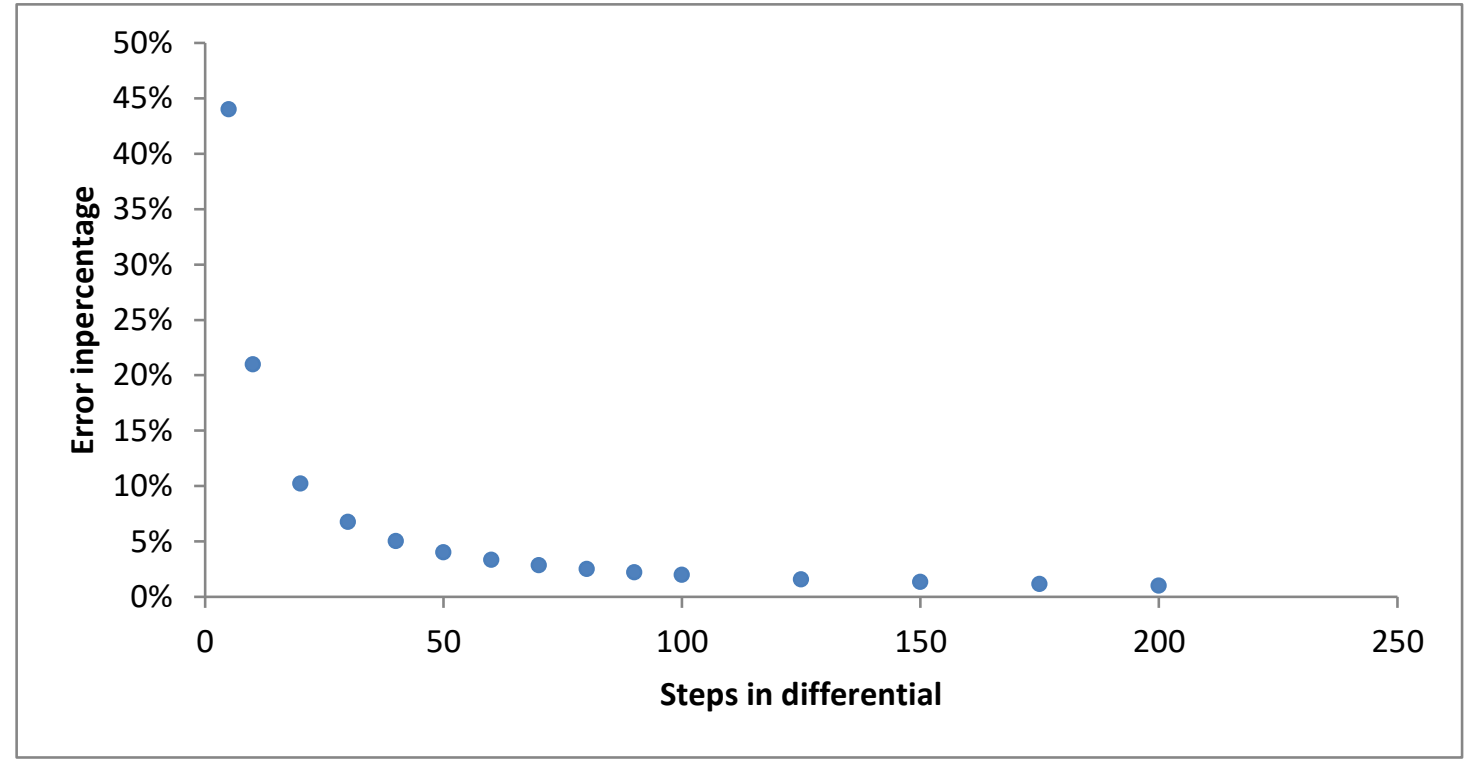

Figure.3. 14. Errors induced (in percentage) by the differential calculation at different differential steps number used in calculation.

Most steps are expected to be calculated in a height difference of less than $0.1 \%$ 
of the probe radius. A calculation gap size check was applied on the vertical direction with the smallest probe size of $5 \mu \mathrm{m}$. The percentage of steps that satisfied the condition are shown in Figure.3.15 for low calculation step number of less than 10000 . At 1000 step calculation, only $70 \%$ of the steps satisfies the preferred calculation condition of height difference less than $0.1 \%$ of the probe radius. This rapidly increases to $98 \%$ when calculation step number increased to 5000. The satisfied percentage is kept increasing with more steps applied in the calculation. From Figure.3.15, it can be as large as $99.5 \%$ when 10000 steps were used in calculation. Ideally, there must be a step number that will guarantee all the calculations satisfy the set condition. An extended calculation showed that it increases to $99.9 \%$ at 20000 steps.

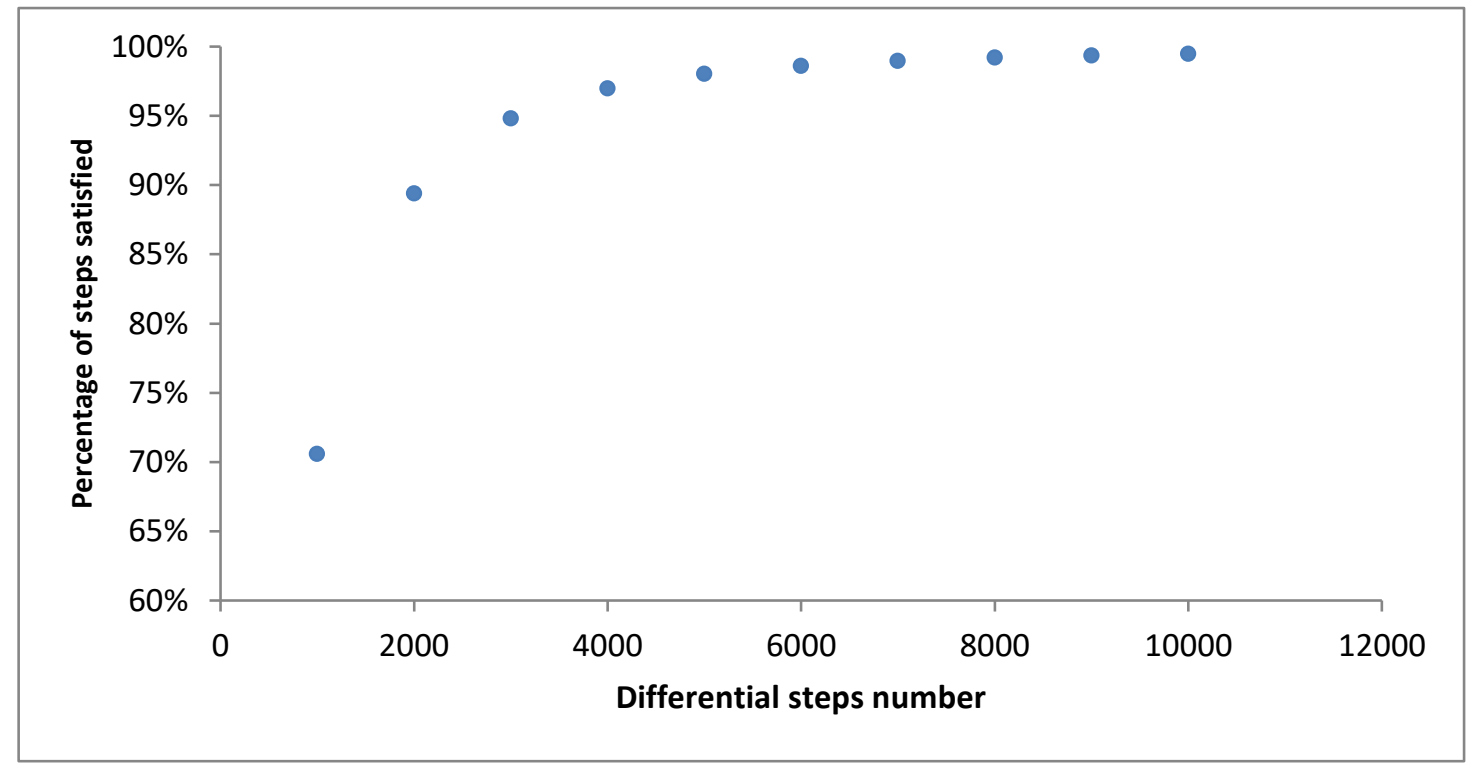

Figure.3. 15. Influence of calculation steps on percentage of vertical gap in calculation satisfied the condition of less than $0.1 \%$ probe radius.

Higher steps number is also checked. Calculation with over 50000 steps are tested still have a few differential steps not satisfy the requirement on height. It is found to be 6 and 4 when the number increased to 100000 and 200000. From the trend of height gap change, the last step will always be the largest step. From the equation of height change (Equation.3.10), the minimum steps required to make all points 
satisfied can be calculate as Equation.3.22.

$1-\frac{1}{n}=1-(0.001)^{2}$

Equation.3.22

Where $\mathrm{n}$ is the number of differential steps. The theoretical minimum steps required from the prediction of Equation.3.22 were calculated as 1 million. It is significant that 1 million steps will make the calculation very slow. In using the whole code, 5000 steps cost around 15 minutes for calculation and about 30 minutes for 10000 steps. Doubling the step number will double the calculation amount, which will half the processing speed. The number was selected in the range of 5000 and 15000 , that is a reasonable size of calculation, which processes the integration in a tolerable speed with good accuracy. The error induced in area was found to be $0.04 \%$ when using 5000 steps in calculation, and this was deemed a negligible level.

\subsubsection{Assessment of Impact of the Influence of Van der Waals Force in the Calculation}

Van der Waals forces are interactions between two surfaces when very close to each other. These forces are also described in DLVO theories and can be approximately calculated. Simulation of overall force change applied when two flat plates with charge approached each other were achieved by Bowen et al. (Bowen et al., 2002), and the calculation code was presented in Stoton's thesis for the corresponding work (Stoton, 2001). To assess the impact of van der Waals forces on the interaction of a colloid probe and a surface as compared to electrostatic interaction Stoton's simulation program was used to calculate the van der Waals force (Figure.3.16).

In different concentrations, the Debye length will be different. The value in 3 ionic strengths was calculated using DLVO theory, and found to be $30.43 \mathrm{~nm}$ in $0.0001 \mathrm{M} \mathrm{NaCl}$ solution, $9.62 \mathrm{~nm}$ in $0.001 \mathrm{M} \mathrm{NaCl}$ solution and $3.04 \mathrm{~nm}$ in $0.01 \mathrm{M}$ $\mathrm{NaCl}$ solution. The change in Debye length with ionic concentration is apparent in Figure.3.16. 


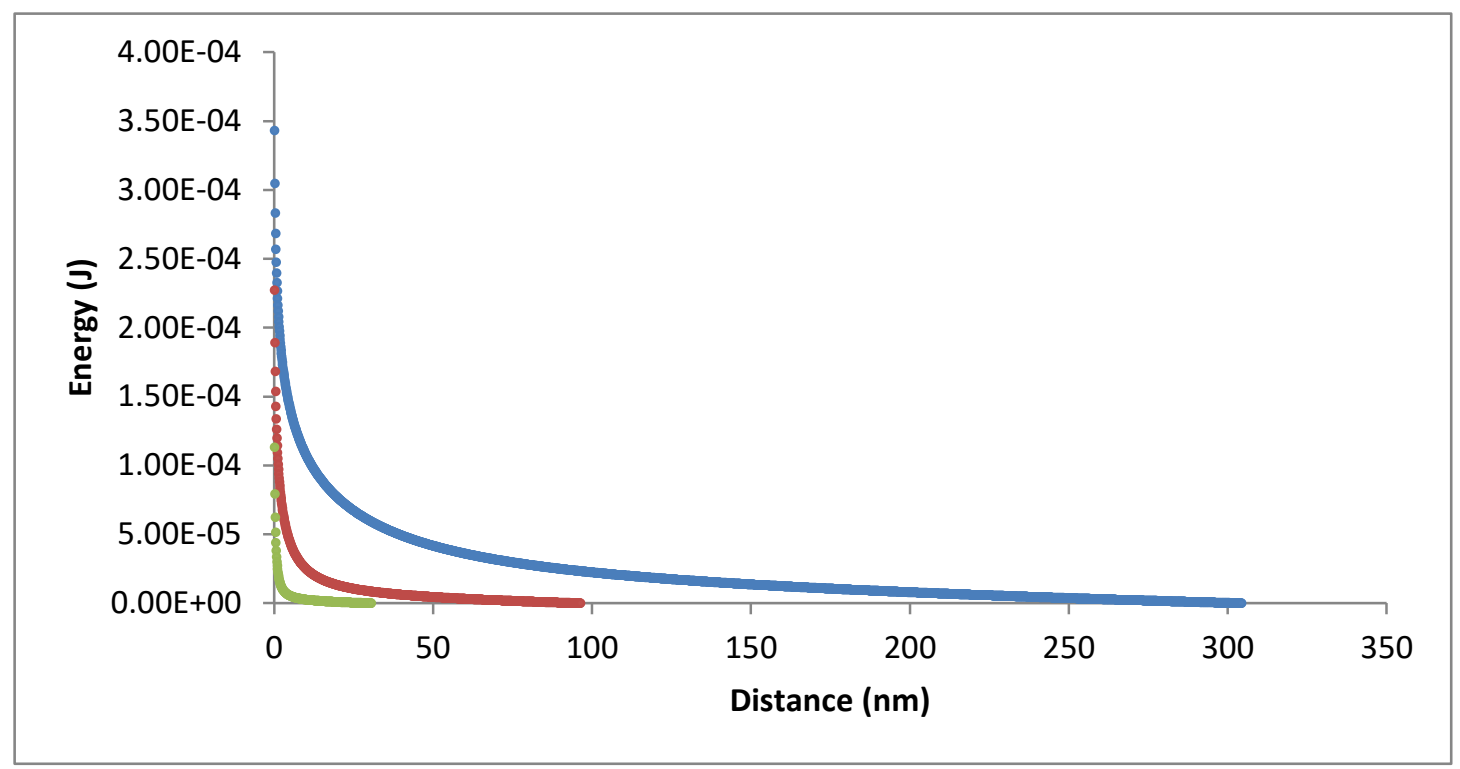

Figure.3. 16. Plot of van der Waals interaction energy change of flat plates approaching to each other from $10 \kappa$ to contact in $\mathrm{NaCl}$ solution of $0.0001 \mathrm{M}$ (blue), $0.001 \mathrm{M}$ (red) and $0.01 \mathrm{M}$ (green).

The simulations using Stoton's code also showed the van der Waals interaction changes more significantly when close to surface. For example, most energy is applied within $1 \kappa$ as displayed in the simulation plot of plates in $0.0001 \mathrm{M} \mathrm{NaCl}$ solution in Figure.3.16. In the same plot, the energy increasing from $1 \kappa$ separation to contact is 6 times, and energy at contact is about 10 times larger than the energy at $2 \kappa$ separation.

The FORTRAN code of this thesis was used to calculate electrostatic force for comparison with levels of van der Waals forces (Stoton's program) within $10^{-4} \mathrm{M}$ $\mathrm{NaCl}$ solution (Figure.3.17) between two flat plates. The energy at $2 \kappa(0.0000356 \mathrm{~J})$ is also found to be smaller than the electrostatic potential at the surface $(0.00004 \mathrm{~J})$. Thus, at this position, electrostatic forces are more significant than van der Waals forces.

In this work, the contact position was regarded as when the two double layers overlapped, and so the suitable position to calculate van der Waals forces to show impact $2 \kappa$. Therefore, if we plot both electrostatic energy and van der Waals energy from $2 \kappa$ to $10 \kappa$, it will present a comparison of electrostatic force and van der Waals force, as shown in Figure.3.17a. The change of electrostatic force is found to be much 
more significant than the change on van der Waals force.

The electrostatic force reduction is in an exponential relationship with the separation distance. A comparison of log electrostatic energy and log van der Waals energy is shown as Figure.3.17b. The reduction of van der Waals force was found to increase when the two surfaces were far from each other.

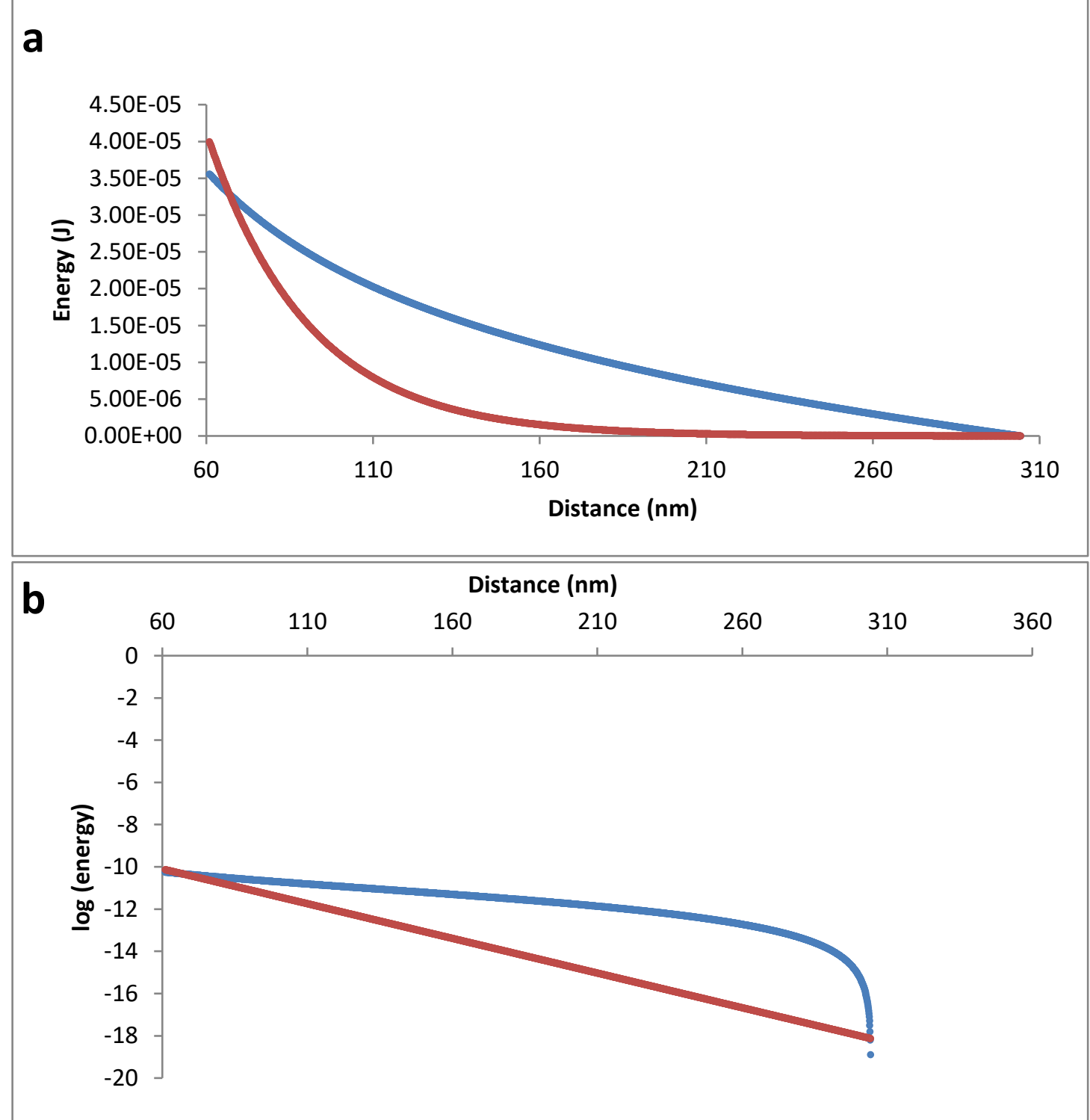

Figure.3. 17. Double layer considered comparison of electrostatic force and van der Waals forces (a) and its log plot (b) for $40 \mathrm{mV}$ plates in $0.0001 \mathrm{M} \mathrm{NaCl}$ solution, blue for Stoton's simulation from $2 \kappa$ and red for DLVO electrostatics estimation from double layer start overlap. 

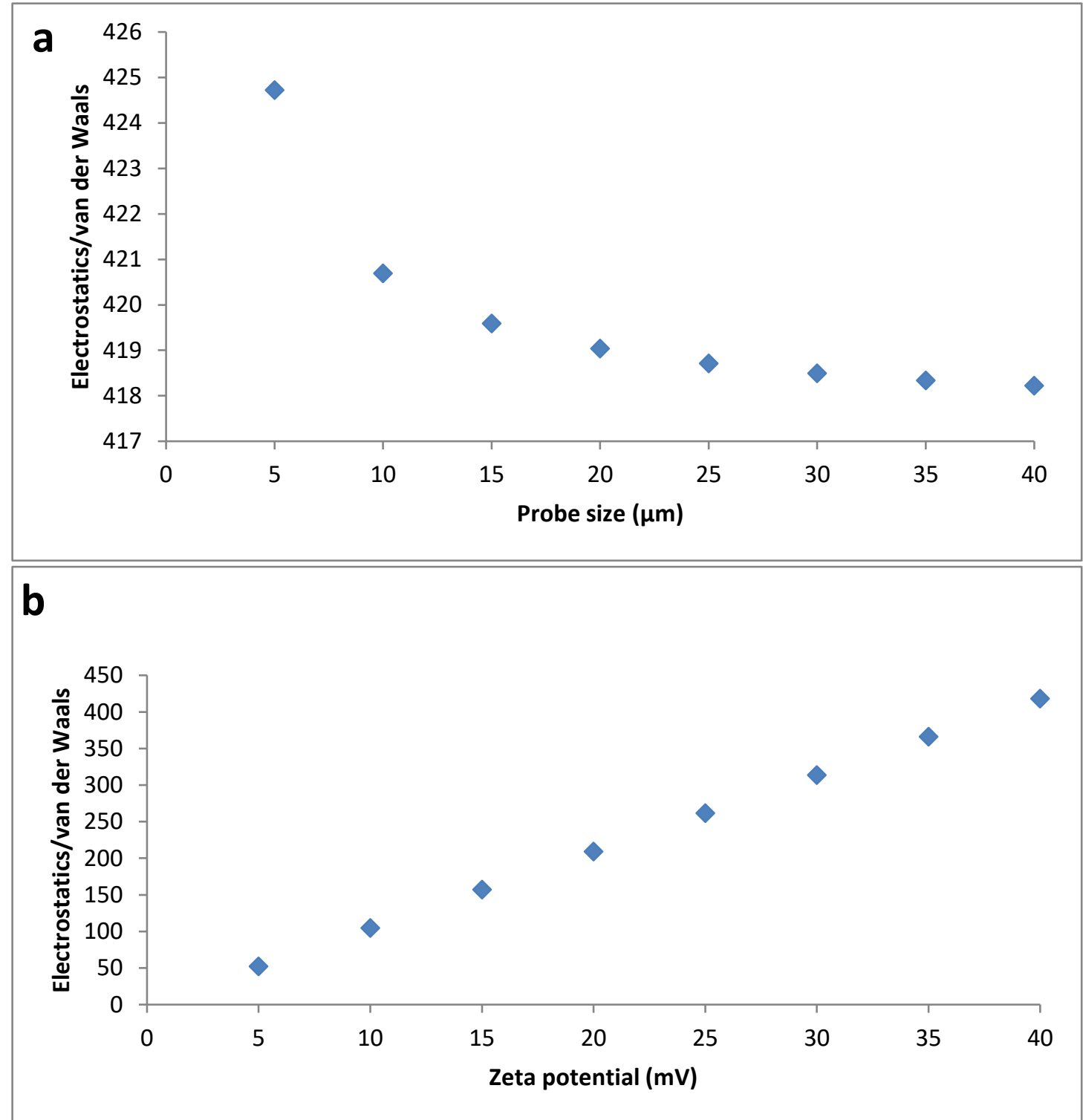

Figure.3. 18. The influence of probe size (a) in constant $40 \mathrm{mV}$ potential and zeta potential (b) in constant $40 \mu \mathrm{m}$ diameter probe size on ratio between electrostatic force and van der Waals forces.

From Figure.3.17, the influence of van der Waals forces is significant compared to electrostatic force when separation distance is constant. However, a colloid probe was used in this study and not two flat plates. The sample can be considered as a flat surface, the probe surface is approximately a sphere. It means that most of the surfaces are far from each other because of the geometry of the probe, even for the smallest probe used ( $5 \mu \mathrm{m}$ diameter) that was much larger than the scale of 10 Debye lengths $(310 \mathrm{~nm}$; Figure.3.17a). The calculated energy was applied to the surface area 
differential calculation to compare the overall influence of van der Waals force and electrostatic force. From the analysis of Figure.3.16, the smaller ionic strength will create a larger effect of van der Waals forces. Thus, the smallest ionic strength calculated in $0.0001 \mathrm{M}$ was used for calibration. Logically, a smaller surface charge density will induce smaller electrostatic forces, so $0.001 \mathrm{C} / \mathrm{m}^{2}$ was selected for calibration as this value was smaller than the lowest surface charge density $(0.001098$ $\mathrm{C} / \mathrm{m}^{2}$ ) in zeta potential calculation.

Comparison of electrostatic force and van der Waals force for different colloid probe diameter sizes and constant surface charge was plotted in Figure.3.18a. (The van der Waals force was calculated from Stoton's code for the appropriate distance for each segment of the colloid probe differentiation of the electrostatic potential determination.) The van der Waals force was found to be more significant when probe size becomes larger. Even larger probe size will better reflect the effect of van der Waals force, electrostatic force is still 418 times of van der Waals force for the $40 \mu \mathrm{m}$ probe diameter size. In this research, the largest probe used is about $35.5 \mu \mathrm{m}$. For the same zeta potential, probe surface charge density and ionic strength, the percentage of van der Waals force size will always be less than $0.25 \%$ of the electrostatic force at contact position.

Van der Waals force is a more constant force compared to electrostatic force, it is not very influenced by probe surface charge density and surface zeta potential. Probe surface charge density was set to be smaller than the minimum used, so the influence of surface charge density can be guaranteed always larger than its maximum when calibrating. The effect caused by small zeta potential was verified using an extra condition of $40 \mu \mathrm{m}$ probe size as a large probe size that shows more significant van der Waals force. The verification results were plotted as Figure.3.18b. The smaller zeta potential shows more significant van der Waals forces. The smallest zeta potential calculated with the program when analysing measured AFM force curves was $5 \mathrm{mV}$. From Figure.3.18b, when zeta potential is $5 \mathrm{mV}$, electrostatic force was found to be 52.3 times greater than van der Waals forces. The maximum van der Waals force influence is found to be less than $2 \%$ of electrostatic force effect with the 
lowest ionic strength, largest probe size, smallest probe surface charge density and smallest sample surface zeta potential.

From the verification, the maximum van der Waals force effect is less than $2 \%$ of electrostatic force influence in the worst-case scenario. Van der Waals force was a negligible force at the position of zeta potential calculation. Thus, in balance the present study decided that to avoid further calculation and code complexity, it was legitimate to avoid van der Waals force. Van der Waals force may also be directly observed from approaching force curves, as it will become significant when surfaces are close to each other (as that found in section.4.5.2). Theoretically, it will not be as significant as the values shown in Figure.3.16, because the surfaces coming into contact in this program are a spherical and flat surface. There may also be obstruction by other factors, such as surface roughness and chemical bonding. Surface forces at peaks of the roughness may start influencing the interaction before van der Waals force become significant enough to be recorded by AFM. At most surface of this research, roughness over the size of Debye length was found and most these approaching force curves are now showing a significant attractive force, as shown in Figure.3.19a. Some force curves are also found to be with a significant snap-in, as shown in Figure.3.19b. According to the some of the dispatching force curves were with a significant adhesion force. It is hard to identify if it is a van der Waals force or a chemical bonding.

Described as the verification, van der Waals force is less effective when calculate zeta potential. Because its influence is in limited range and weakened by the measuring condition. The coding system will not focus on van der Waals force and more general analysis from approaching force curves observation is a more reasonable method.

Review of previous research that has studied van der Waals forces with AFM showed that the experimentation required rigid control of the surface morphology, which is not commensurate with the objectives of this study; flat sample surfaces are required, such as mica (Butt, 1991) and crystal structures (Kuhn \& Rahe, 2014). The van der Waals influencing distance was found to be short at less than $20 \mathrm{~nm}$ and 
chemical forces are not considered or only considered over a short-range (Butt, 1991; Harimawan et al., 2013; Kuhn \& Rahe, 2014).

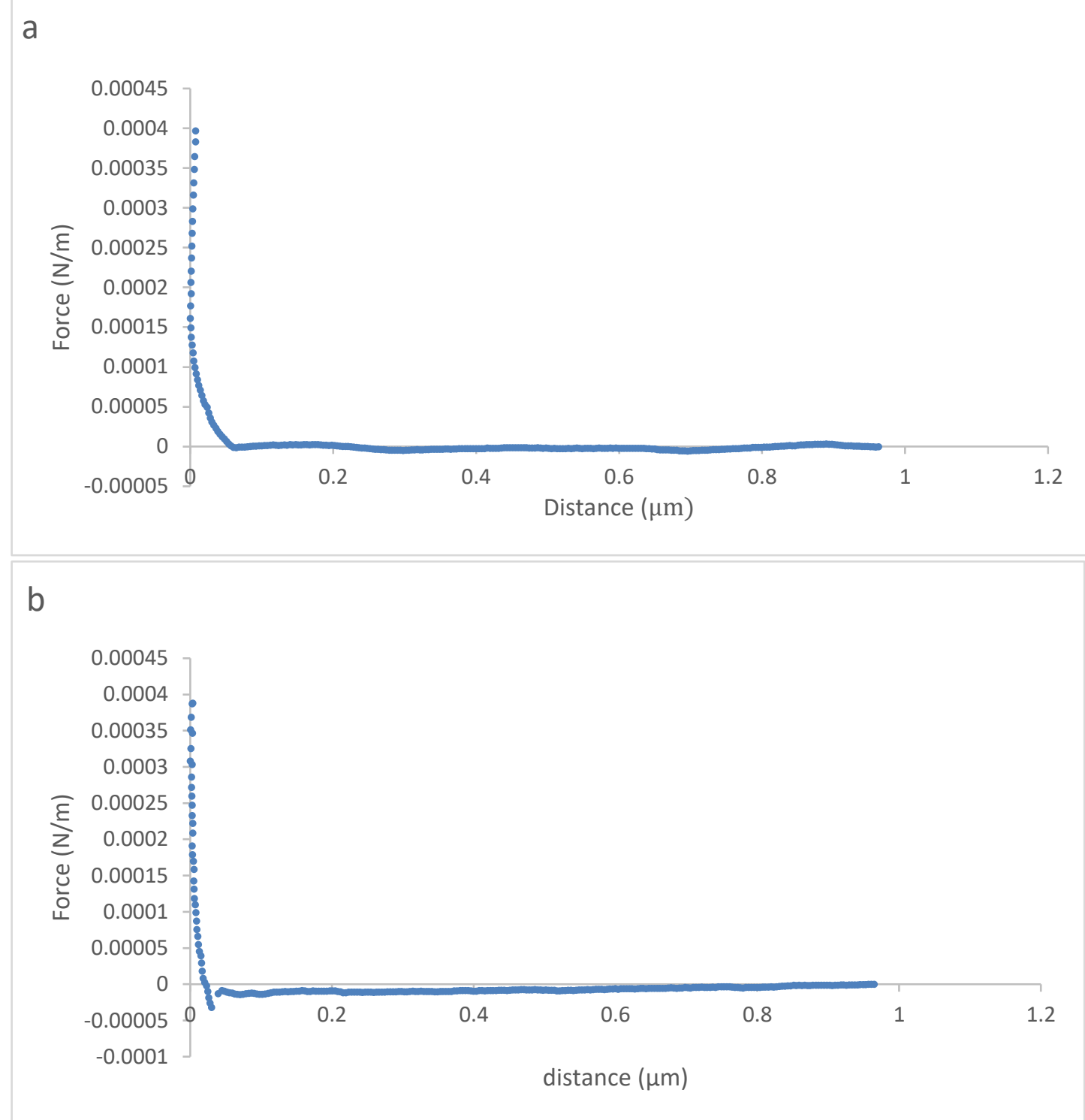

Figure.3. 19. Treated AFM approaching force curve without a snap in (a) and with a snap in (b).

\subsubsection{Validation and Calibration of Zeta Potential Calculation}

The calculation of zeta potential was developed based on the DLVO theories. There are other researchers that have simulated the electrostatic force change depending on different planes of separation distance (Brant et al., 2006). Bowen et al. 
considered linearized electrostatic energy in their simulation of interactions between two ideal flat plates as applied to membrane surface characterization (Bowen et al., 2002). Their established simulation was used to verify the zeta potential calculation from AFM force curves, an objective of this thesis.

From Stoton's work (Stoton, 2001), the electrostatic energy is linearized, which means the energy calculated with their code will always have an error compared to DLVO calculations, as shown in Figure.3.20. In Figure.3.20, both curves start and end at the same points with negligible differences.

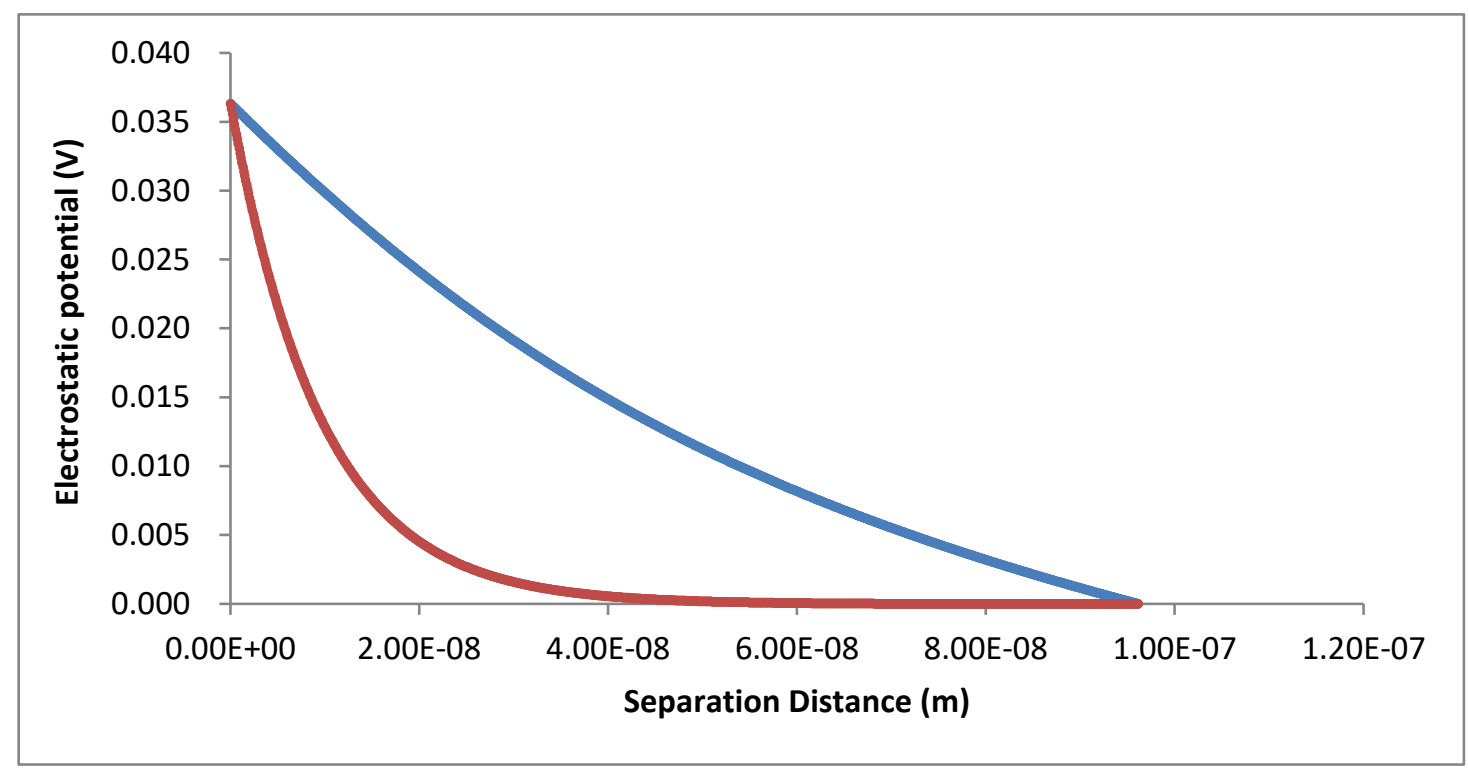

Figure.3. 20. Comparison of the estimation of electrostatic potential at different separation with DLVO theories (red) and Stotons's linearized simulation (blue) (Stoton, 2001).

Theoretically, the electrostatic potential calculated with each point from the simulation will result in the same zeta potential. The code from Stoton's work is used to simulate a series of electrostatic potential reduction curves in different ionic strength and plate surface potential (Stoton, 2001), Zeta potential of both plates are originally set as the same for easier calculation. According to the principles of DLVO estimations and Stoton's simulation, there must be an error if the zeta potential is not calculated with the starting point or ending point, as shown in Figure.3.21. 


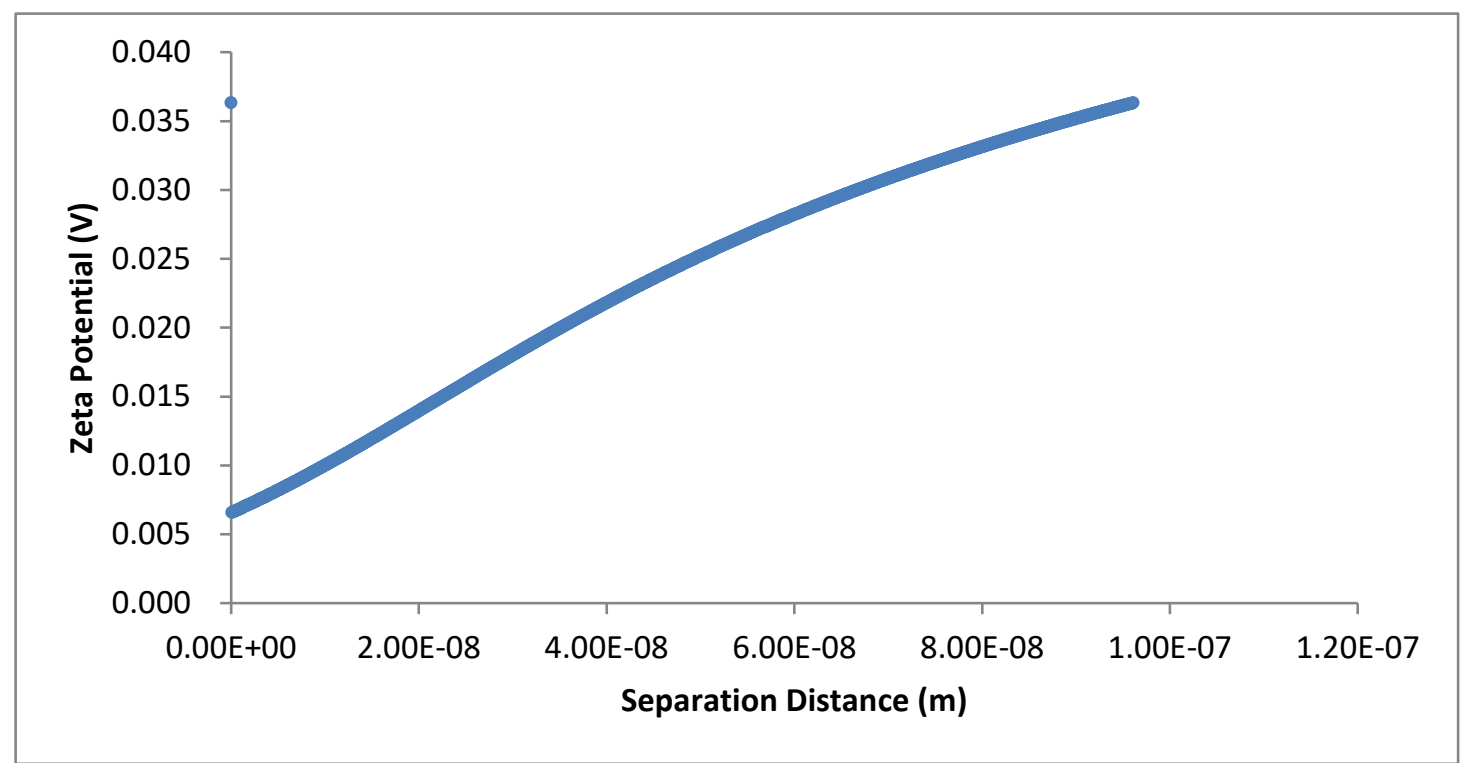

Figure.3. 21. Zeta potential calculated with the electrostatic potential calculated at different separation distances with Stoton's electrostatic potential simulation (Stoton, 2001) curve with $0.036 \mathrm{~V}$ plates in $0.0001 \mathrm{M} \mathrm{NaCl}$ buffer solution.

$\Psi_{z}=\Psi \exp (\kappa h)$

Equation.3.23

Where $\Psi_{\mathrm{z}}$ is zeta potential and $\mathrm{h}$ is separation distance. Using Equation.3.21, which is derived from Equation.3.2, the calculated zeta potential differences between DLVO theory and Stoton's simulations at each position of the simulation were shown in Figure.3.21. The error between linearized potential and DLVO estimated potential is obvious, and could be caused by a combined influence of both $\Psi$ and h. To simplify the verification calculation, the influences of $\Psi$ and $\mathrm{h}$ were calculated separately. The zeta potential calculation is based on the potential change when two plates separate from contact to the selected separation distance. From Equation.3.21, the calculation equation can be rewritten as Equation.3.24 with the calculation of electrostatic potential change from the surface to a distance from surface. To simplify the calculation, the exponential term is regarded as $\mathrm{R}$.

$\Psi_{o}=\frac{\Psi}{1-\exp \left(-\frac{h}{\kappa}\right)}=\Psi \mathrm{R}$

Equation.3.24

To understand the influence on $\Psi$ and h, a comparison of Stoton's linearization error in zeta potential calculated from simulation and electrostatic potential difference between simulation and DLVO estimation was plotted as Figure.3.22a. The 
exponential term difference between simulation setting and DLVO recalculation was plotted in Figure.3.22b. Both plots showed more significant difference when the two plates are close to each other. This feature also explains why more error was apparent in the zeta potential calculated when two plates are closer, as shown in Figure.3.21. More analysis is required to reduce the error induced by linearization.

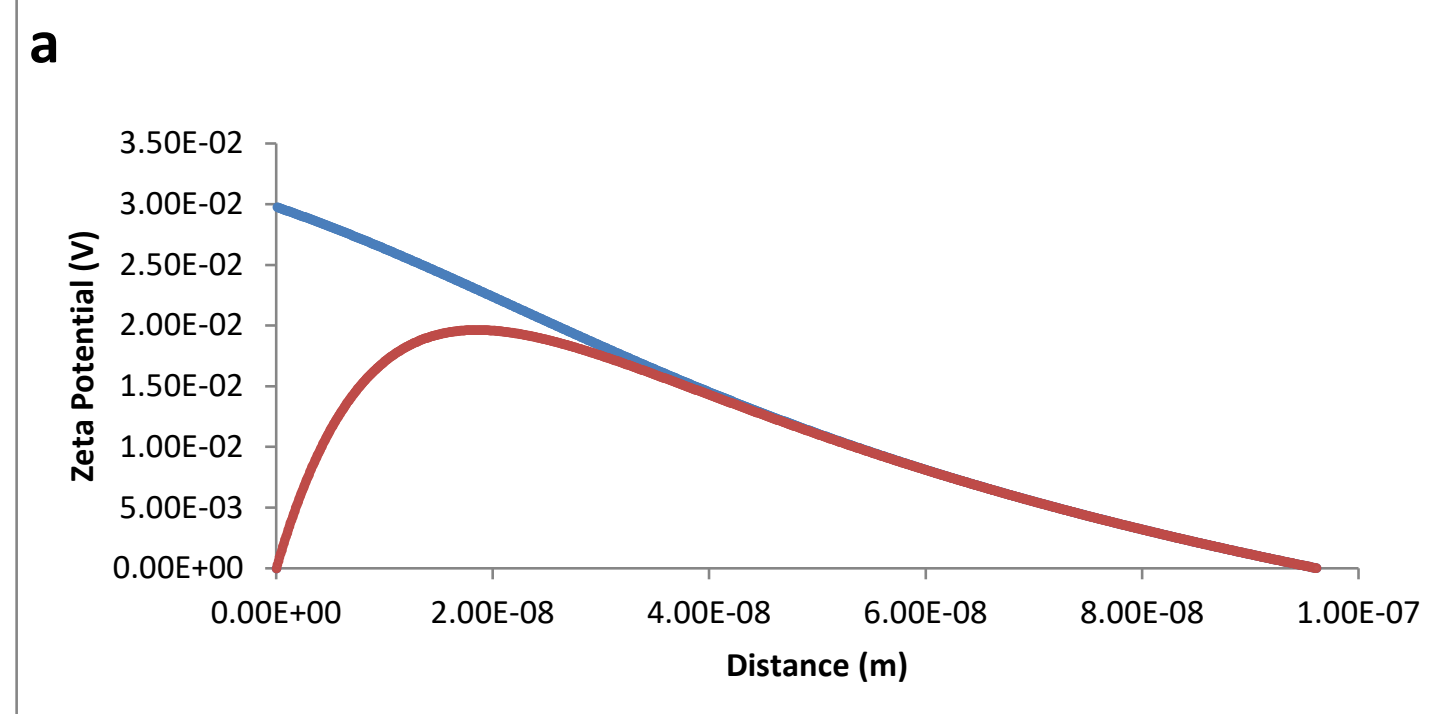

\section{b}

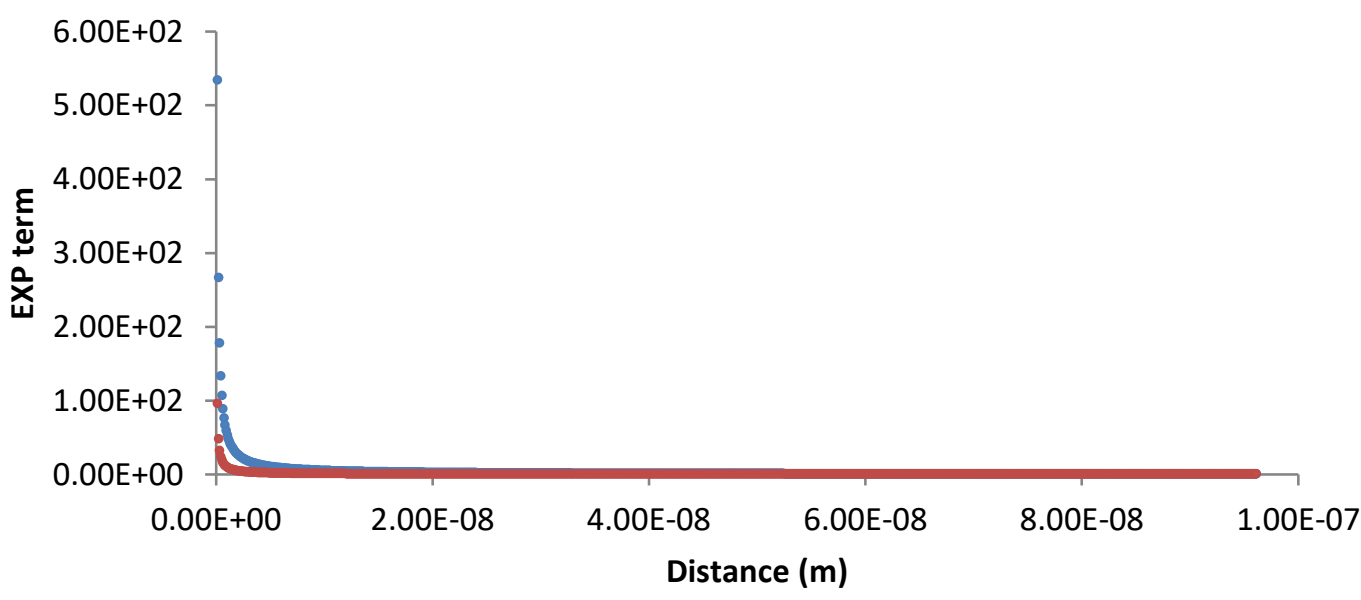

Figure.3. 22. a is comparison of calculated zeta potential error with Stoton's simulated curve (blue) and local potential error to DLVO estimation (red) at different separation distance, $\mathrm{b}$ is the exponential comparison of Stoton's simulation (blue) and simulation setting separation (red). Calculation set is $0.0036 \mathrm{~V}$ plates in $0.0001 \mathrm{M}$ $\mathrm{NaCl}$ solution.

$\Psi_{o}+\Psi_{o e}=\left(\mathrm{d} \Psi+\Psi_{e}\right) R=d \Psi\left(\mathrm{R}+R_{e}\right)$

Equation.3.25 
$\Psi_{o e}=\Psi_{e} R=d \Psi R_{e}$

Equation.3.26

The calculation with error is described in Equation.3.25, where $\Psi_{\mathrm{e}}$ is error in electrostatic potential, $\Psi_{\mathrm{oe}}$ is the error in calculated zeta potential and $\mathrm{d} \Psi$ is electrostatic potential change from two plates in contact to the separation used in zeta potential calculation. To simplify error analysis, the error in separation distance is converted to error in the exponential term $\left(\mathrm{R}_{\mathrm{e}}\right)$. Then, the error in electrostatics can be calculated with Equation.3.26.

Theoretically, the error observed from comparison of DLVO and Stoton's simulations should be the same as the error calculated from error estimation on both electrostatic potential and separation distance with Equation.3.26. From the plot in Figure.3.23, the simulation error (Figure.3.23a), electrostatic error effects (Figure.3.23b) and separation error effects (Figure.3.23c) are found to be the same shape and scale. It means the error analysis is working in the set condition of $0.036 \mathrm{~V}$ plates in $0.0001 \mathrm{M} \mathrm{NaCl}$ solution.

In the zeta potential calculation of the study, zeta potentials in the range from 5 to $40 \mathrm{mV}$ were applied, and measurements were in ionic strengths of $0.1 \mathrm{M}, 0.01 \mathrm{M}$, $0.001 \mathrm{M}$ and $0.0001 \mathrm{M}$ (measured in DI water as buffer). Calculation in different experimental conditions were checked as well. To verify the error, zeta potential calculations with the consideration of error are applied in simulation at $5 \mathrm{mV}, 20 \mathrm{mV}$ and $40 \mathrm{mV}$ in ionic strength of $0.0001 \mathrm{M}$. The calculated zeta potentials from these curves were plotted in Figure.3.24, and simulating in other ionic strengths were also showing similar zeta potential results as that simulated at $0.0001 \mathrm{M}$. It is hard to identify any difference from different experimental condition used. Therefore, only the plot in $0.0001 \mathrm{M}$ solution is applied in Figure.3.24 as an example. Zeta potentials calculated at different points from the same simulation curve were observed to be constant for all 3 zeta potentials. Thus, the calculation is feasible to applied in the validation of zeta potential calculation. The verification code will result constant zeta potential same as setting zeta potential in simulation at every point of the simulated curves. 

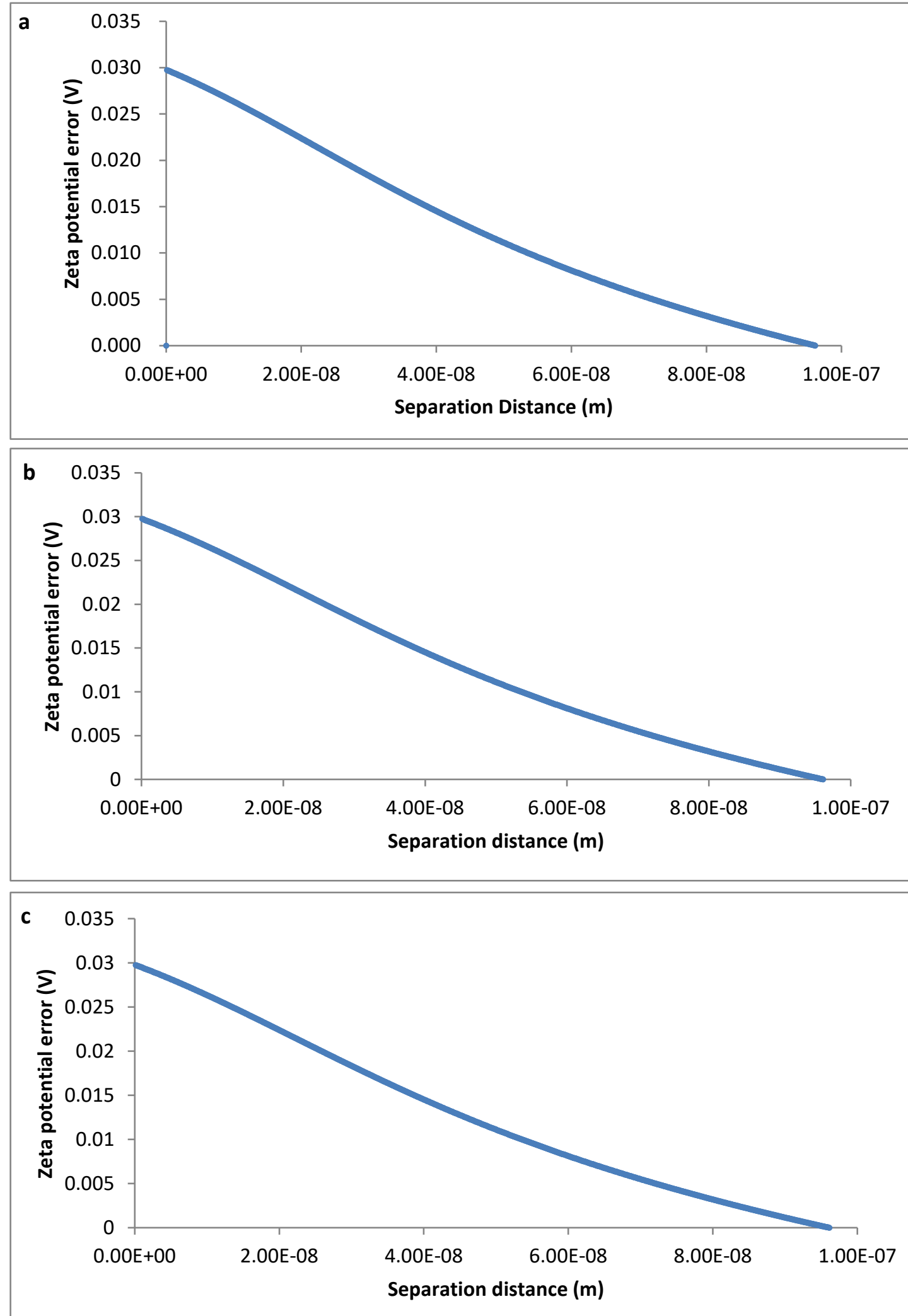

Figure.3. 23. Comparison of zeta potential error observed from simulation (a), calculated with error effects on electrostatic potential (b) and influence on separation distance (c). 


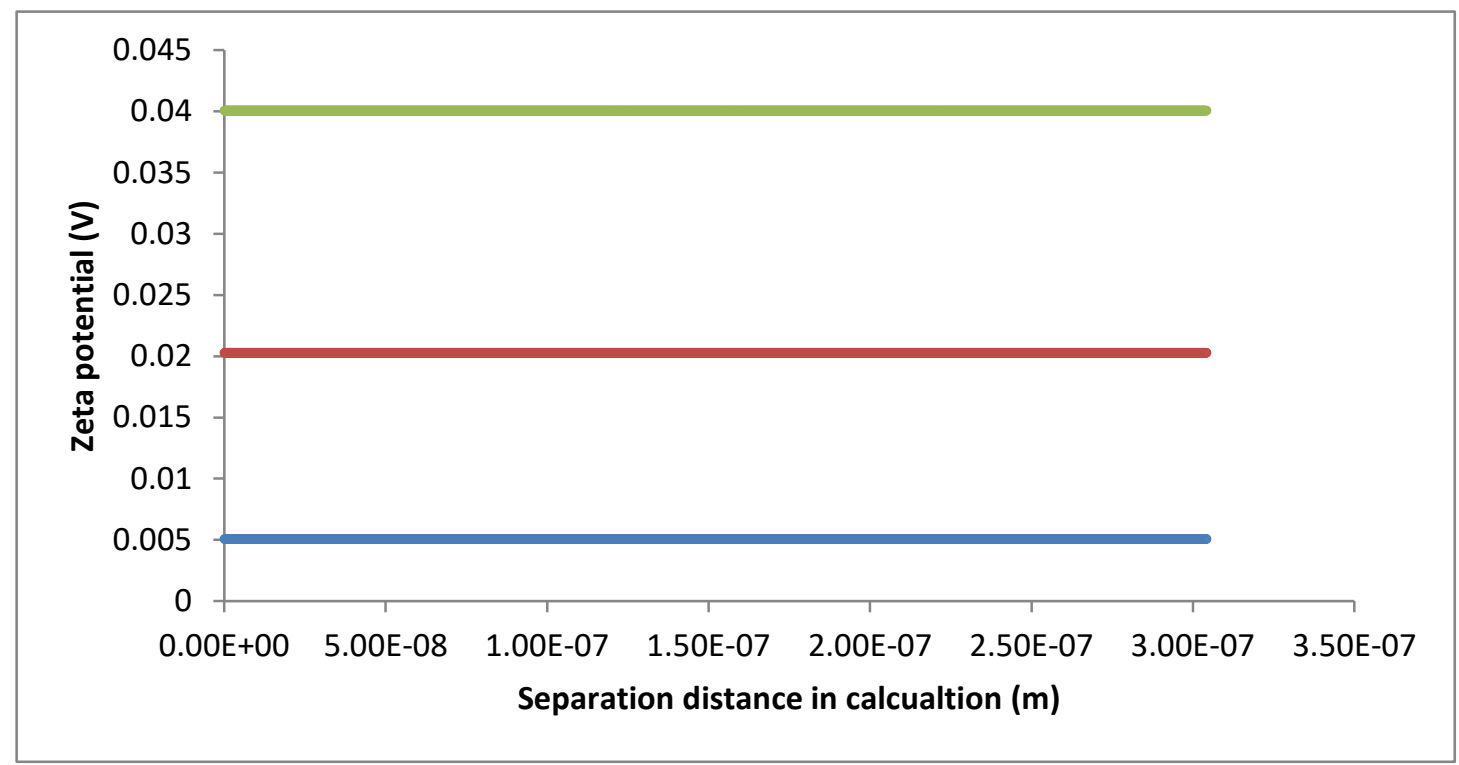

Figure.3. 24. Zeta potential calculated with error reduced simulation curves in $0.0001 \mathrm{M} \mathrm{NaCl}$ solution and $5 \mathrm{mV}$ (blue), $20 \mathrm{mV}$ (red) and $40 \mathrm{mV}$ (green) plates.

From the calculation results with simulated curves, a small difference is found between the initial set estimate and the calculated zeta potentials, for example, calculation of $20 \mathrm{mV}$ plates in $0.01 \mathrm{M}$ solution result a zeta potential of $20.3 \mathrm{mV}$. The difference is presented more clearly in Figure.3.25 for calculations at the same zeta potential in different ionic strengths. The error in $5 \mathrm{mV}$ (Figure.3.25a), $20 \mathrm{mV}$ (Figure.3.25b) and $40 \mathrm{mV}$ (Figure.3.25c) plates in different solutions can be clearly observed.

This error due to the linearization could be brought in by the surface charge density calculation, as the method is developed from the linearized potential calculation. In consideration of accuracy, it is a combination of the linearization calculation and Poisson-Boltzmann Equation (PBE). Calculation error (due to linearization) in this section is negligible, and a plot of the error shows that it is at a reasonable level for this calculation (Figure.3.26). These errors are found within the range of $\pm 2 \%$ to the set value and appears randomly applied in the calculated values. 

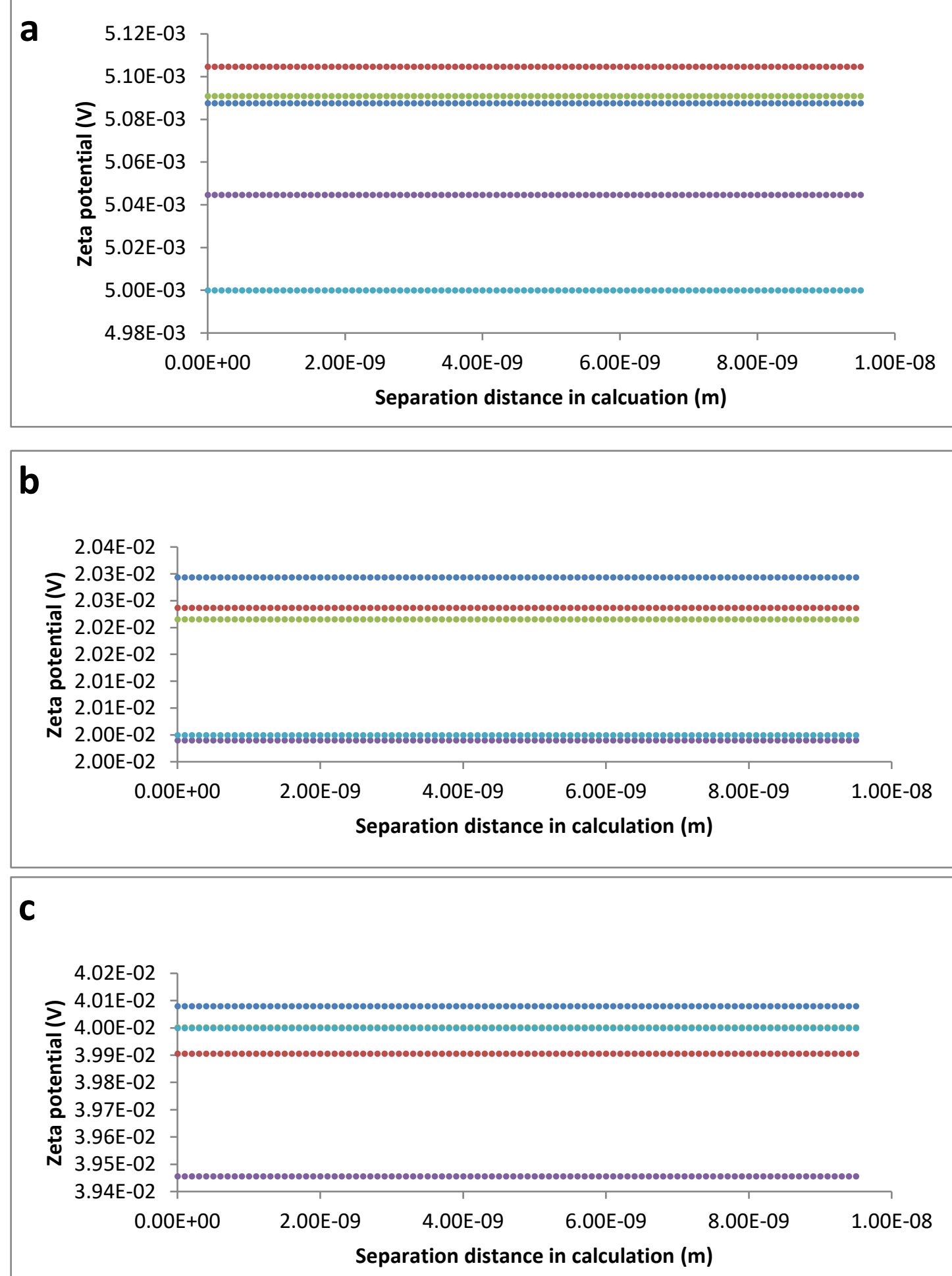

Figure.3. 25. Zeta potential calculated with error reduced simulation curves in set zeta potential of $5 \mathrm{mV}$ (a), $20 \mathrm{mV}$ (b) and $40 \mathrm{mV}$ (c) in ionic strength of $0.0001 \mathrm{M}$ (blue), $0.001 \mathrm{M}$ (red), $0.01 \mathrm{M}$ (green) and $0.1 \mathrm{M}$ (purple) $\mathrm{NaCl}$ solutions compare with set zeta potential in simulation (cyan). 


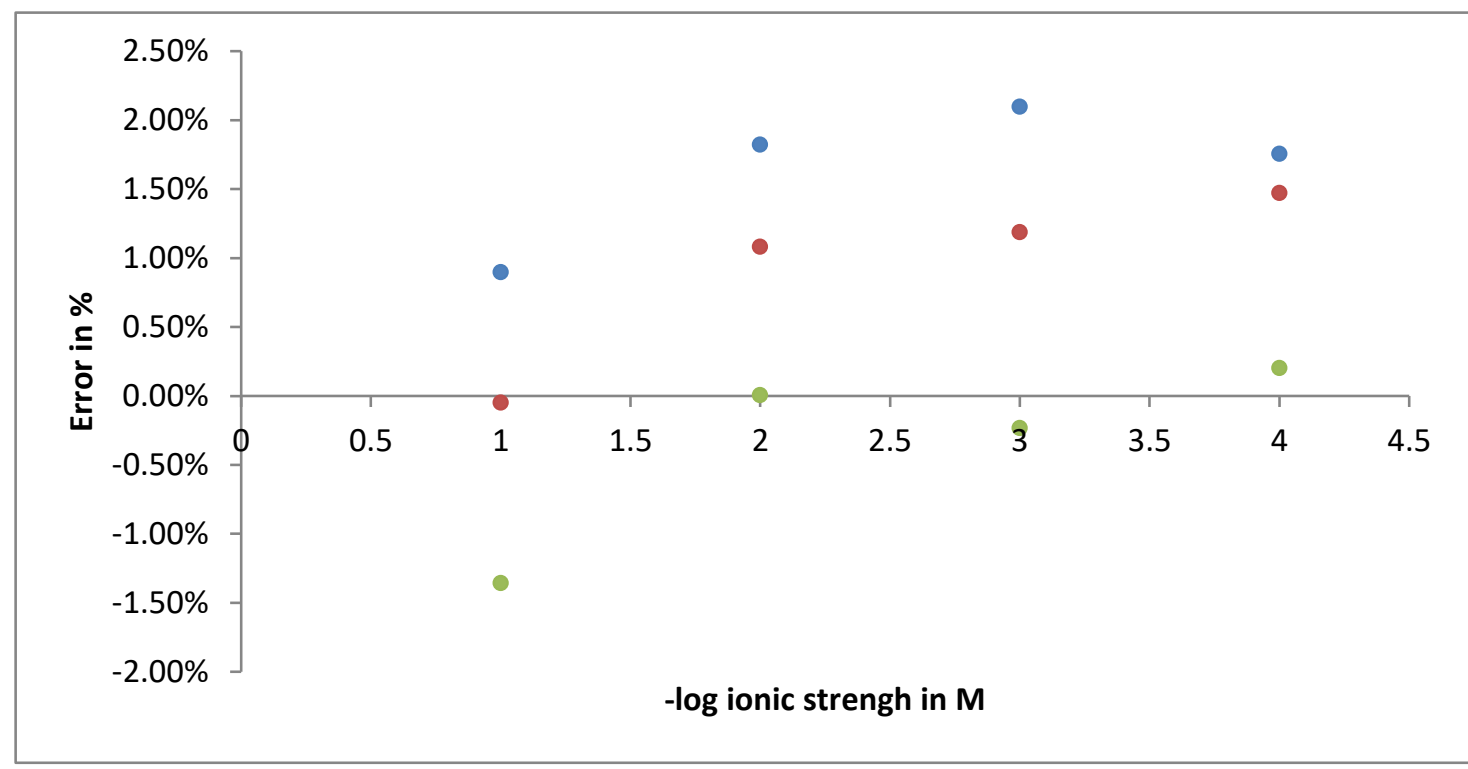

Figure.3. 26. Error induced by the surface charge density calculation at $5 \mathrm{mV}$ (blue), $20 \mathrm{mV}$ (red) and $40 \mathrm{mV}$ (green) in different ionic strength. Ionic strength was plot in the form of $-\log$ for better illustration.

With the error analysis on zeta potential calculation, the calculation will not generate errors. Error is brought in by the calculation of surface charge density. An overall error of around or less than $2 \%$ is observed in this section. Compared to the errors in differential calculation and van der Waals forces influences, it is larger but still in a tolerable level.

\subsubsection{Code Precision Validation with Consideration of All Error Induced}

In the error analysis, errors are induced from both the differential calculation on the probe surface and the zeta potential calculation. The influences of van der Waals forces will also have created an error that will affect the result. When assessing these errors separately, they all remained at a negligible level even in the worst case.

To guarantee accuracy of the calculation, a worst-case study was applied to verify the overall effect of these 3 errors. The maximum error induced by van der Waals level will never exceed $2 \%$, while it is far less than this level in most cases. Differential calculation at minimum steps number of 5000 induced an error of $0.04 \%$ and the error generated by surface charge density calculation is found to be around or less than $2 \%$. With a simplified illustration of the DLVO theory, the calculation is as 
Equation.3.27, where $\mathrm{A}$ is area, $\rho_{\text {charge }}$ is surface charge density.

energy $=\int_{0}^{r} A \Psi_{o} \rho_{\text {charge }} \exp \left(-\frac{d}{\kappa}\right) d r$

Equation.3.27

From Equation.3.27, the influence of the 3 errors can be determined as the overall error. In consideration of the worst-case, the errors were all set to be its maximum values for calculation, and the calculation can be simplified to Equation.3.28. Where error1, 2 and 3 presented the maximum values of 3 errors found.

error overall $=(1+$ error 1$)(1+$ error 2$)(1+$ error 3$)-1 \quad$ Equation.3.28

When the error maximum values are used, 2\% from van der Waals force, 2\% from surface charge density calculation and $0.04 \%$ from surface differential calculation, the overall error limit is found to be $4.08 \%$. The overall error limit is less than $5 \%$, which is considered as the critical value which causes significant influence. In most cases, errors will be much smaller than the $4.08 \%$ calculated in worst-case study, which means the overall error will be smaller. Thus, providing confidence in the precision of the calculation when processing measured AFM force curves. Further, validation of the method is achieved in section.4.3.3 when the zeta potential measurement achieved through force-distance curve analysis is compared with previous research and streaming potential measurements.

\subsection{Development of Hydrophobicity Analysis Model}

\subsubsection{Solvent Drag Force Analysis of Cells Partitioned in the Different Phases}

In solvent partitioning of particles to study their hydrophobicity, the solvent drag force is a concept that accounts for all the forces that are acting at the surface of the particle to maintain the population of particles within the buffer solution as opposed to the polar solvent. The hydrophobicity (OD) of a microbial cell population is the ratio of spectrophotometer measured light intensity before and after washing by hexadecane. The light intensity change is approximately linearly related to the density of microbial cells (Beer-Lambert law) remaining in the sample solution (Beers \& Sizer, 1951). Therefore, the OD measured is the percentage of microbial cells 
remaining in the buffer solution. There are many kinds of forces applied on a microbial cell that affect the cell as to whether it remains in the buffer solution, for example, hydrophobic interaction, hydrophilic interaction and a physical force such as gravity, van der Waals forces and electrostatic repulsion (Pfreundschuh et al., 2014).

The dead cell population also provide a constant influence on OD measurements. The size of a dead cell is the same as a live one, but dead cells will have different surface chemical properties and will not be sensitive to buffer conditions that impact biologically at cell surfaces. Thus, the dead cell population will influence OD measurements.

However, the dead cell percentage can be regard as the same for all samples, as the culturing media, condition, time and cell wash conditions are all the same. The consistent experimental conditions of the same temperature and pressure will provide constant thermodynamics, pressure gradient and gravity. In addition, intermolecular forces are not significant to large particle such as microbial cell, because the distance between cells are relatively large when suspended in buffer. Therefore, these forces can be regard as a constant force in all samples, and it will be approximately a constant for all measurements. There are also some unreactive hydrophobic or hydrophilic groups on cell surfaces (Hermens et al., 1984). These are also considered as constant force that will not affected by buffer and measurement conditions. From the OD test experience throughout the study, only $90 \%$ of the light intensity after mixing is caused by live cell; $10 \%$ of the population remained in the hexadecane, these were assumed to be dead cells as when cells die they lose their hydrophilic proteins at their surface exposing more hydrophobic regions of their wall structure (Majno \& Joris, 1995). Thus, the OD that is caused by live cells can be calculated as Equation.3.29.

$$
\begin{array}{ll}
O D_{\text {real }}=\frac{O D_{\text {test }}}{90 \%} & \text { Equation.3.29 } \\
F_{\text {total }}=F_{\text {hydropilic }}-F_{\text {hydrophobic }}+F_{\text {constant }} & \text { Equation.3.30 }
\end{array}
$$

For a live cell population, the hydrophobic and hydrophilic force is more effective for determining the cell numbers suspended in buffer or hexadecane. Salt 
concentration and $\mathrm{pH}$ will provide ions of $\mathrm{H}+, \mathrm{Na}+, \mathrm{OH}-$ and $\mathrm{Cl}-$, which will be potentially reactive to hydrophobic or hydrophilic chemical groups on microbial cell surfaces. To define the cell population suspending in buffer or hexadecane, Equation.3.30 is used to identify the total drag force on the cells in buffer. As shows in Equation.3.30, cells will be remaining in the buffer if total force is positive. Otherwise, cells will be washed away by hexadecane.

\subsubsection{Cell Surface Hydrophobicity Analysis of Cell with Reaction Equilibriums}

The buffer drag force acting on a cell is highly related to the hydrophobic and hydrophilic chemical groups on the cell surfaces. Even in the same culturing conditions, the density of these chemical groups on cell surfaces are randomly distributed around the average value. Normal distribution can be applied to analyse the distribution of these effective chemical groups.

In this study, the OD test of cells is providing a high optical density with results normally larger than $50 \%$. It means either the cells contain a much higher average of hydrophilic chemicals than the hydrophobic groups or the cell surface is rich in unreactive hydrophilic chemicals. Hydrophobicity of cells is affected by $\mathrm{pH}$ and buffer concentration and experimental OD results will show the difference. Therefore, there are two kinds of chemical reaction that may be occurring on the cell surface that can be mathematically described as in Equation.3.31 - 34. Both ionic strength and $\mathrm{pH}$ will influence the charged ions density in buffer.

$$
\begin{aligned}
& \text { Surface } \text { Surface } \text { Sution }^{+} \\
& \text {Surface }^{+}+\text {Cation }^{+} \leftrightharpoons \text { Surface } \\
& \text { Surface }^{+}+\text {Anion }^{-} \leftrightharpoons \text { Surface } \\
& \text { Surface }_{\text {Surface }}^{+}+\text {Anion }^{-}
\end{aligned}
$$

Equation.3.34 


\subsubsection{Numerical Analysis of Relationship between Aqueous Phase Optical Density and Cell Surface Hydrophobicity}

The solvent drag force presented in Equation.3.30 shows there are 3 forces to balance the cells in buffer or hexadecane. Therefore, the hydrophobic force can be regard as a constant for cells suspended in the same buffer. Equation.3.30 can be simplified to Equation.3.35.

$$
F_{\text {total }}=F_{\text {hydropilic }}+F_{\text {constant }} \quad \text { Equation.3.35 }
$$

The hydrophilic groups normally exist on the surface proteins, and other non-membrane structures. A Gaussian distribution is applied to analyse the surface density of hydrophilic chemical groups on microbial cell surfaces. The percentage distribution is changing with a standard deviation $(\delta)$ and mean value $(\mu)$ as the average. Theoretically, the standard deviation will be a constant for the same $\mathrm{pH}$.

Consider Equation.3.35 and Gaussian distribution, 50\% of the cells will be washed away when $F_{\text {total }}$ is zero. To simplify the analysis, assume the solution is adjust to the real situation from $F_{\text {total }}=0$. Therefore, the distribution curve was initially based on the mean value at a concentration inducing $50 \%$ of the cells to be washed out $\left(\mathrm{C}_{50 \%}\right)$. The $\mathrm{C}_{50 \%}$ will be the critical value that defines whether the cell was washed away or remained in the buffer. The shifting between real surface density and density at $\mathrm{OD}=50 \%$ (Figure.3.27) clearly shows the difference between the cell surface and surface at $\mathrm{C}_{50 \%}$. The shifting value is found as $\mathrm{n} \delta$, so for the real situation, the critical value of cell washed by hexadecane is at the point of -n $\delta$. Ideally, the cells represented as the part of curve with higher surface density than $\mathrm{C}_{50 \%}$ will not be washed from the buffer.

$$
\int_{C-n \delta}^{+\infty} P(c) d c=1-\int_{-\infty}^{C-n \delta} P(c) d c=1-\operatorname{erfc}\left(\frac{n}{\sqrt{2}}\right) \quad \text { Equation.3.36 }
$$

The percentage of cells remaining in the buffer solution was calculated with an integration of Gaussian distribution equation with the amount shifting from $\mathrm{C}_{50 \%}$, Equation.3.36. 


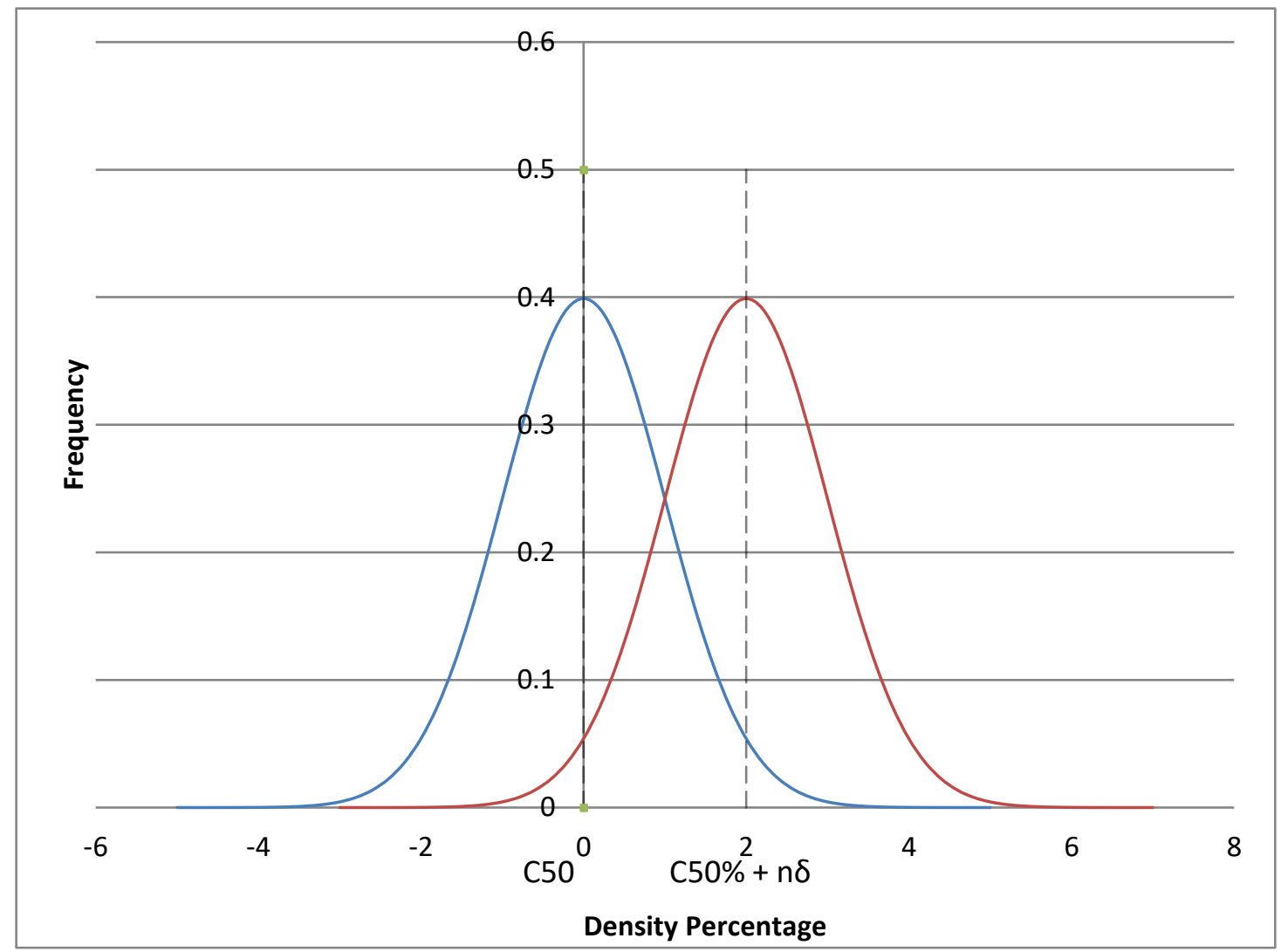

Figure.3. 27. the normal distribution shifting of $n \delta$ with the equilibrium reaction and buffer concentration controlled surface hydrophilic groups density from the test of $\mathrm{OD}=50 \%$.

The shifting number $\mathrm{n}$ can be found with the real OD, which was calculated from test results with Equation.3.29. Therefore, in mathematical development, the $\mathrm{n}$ can be regard as a known value from test results. Many inevitable errors are induced in the spectrophotometer test, there are still errors on the finial OD even with the error minimization consideration of hexadecane and aggregation effects. Due to the character of Gaussian distribution, less effect will be made on the percentage when the critical value is far from the mean surface density measured. Data at these points near to saturation will contain a huge error. Thus, only the OD with significant change is practical for the calculation. A correction of the measured data can be achieved with the proposed model, and it was applied in data analysis that is used in the hydrophobicity analysis of cells in Chapter V. 


\subsubsection{Numerical Conversion of Gaussian Distribution to Determine the Cell}

\section{Surface Hydrophilic Density in the Reaction Equilibrium}

An equilibrium reaction on cell surface (Equation.3.37) can applied in the calculation, which incorporates the equilibrium constant $\left(\mathrm{K}_{\mathrm{E}}\right)$ into the model. The surface density can't be measured with spectrophotometer. Thus, the percentage of free chemical groups $\mathrm{x}$ (can be calculated with Equation.3.38) was used to identify potential maximum hydrophilic surface density, and estimate the free hydrophilic chemical group surface density. The reacted group percentage will be (1-x). Equation.3.37 can be rewritten as Equation.3.39. The $\mathrm{x}$ is highly related to the $\mathrm{K}_{\mathrm{E}}$ with Equation.3.40 derived from Equation.3.39.

$$
\begin{aligned}
& K_{E}=\frac{C_{\text {reacted }}}{C_{\text {ion }} C} \\
& x=\frac{C}{C_{\max }} \\
& K_{E}=\frac{1-x}{C_{\text {ion }} x} \\
& x=\frac{1}{K_{E} C_{\text {ion }}+1}
\end{aligned}
$$

Equation.3.40

From the Gaussian distribution, the real surface density of the hydrophilic groups can be defined as Equation.3.41. There is also a related percentage $(\delta \%)$ for the standard deviation value with the linear relationship of Equation.3.38 to $\delta$. Therefore, Equation.3.41 can be developed as a percentage expression of Equation.3.42. The equilibrium will be a constant for all the concentration used in test at the same $\mathrm{pH}$. Combining the two kinds of expression of $x$ defined in Equations.3.40 \& 42, the relationship between $\mathrm{x}_{50 \%}$ and $\delta_{\%}$ can be elaborated as a linear function Equation.3.43 and an unknown equilibrium constant $\left(\mathrm{K}_{\mathrm{E}}\right)$. $\mathrm{x}_{50 \%}$ will also be a constant value at same $\mathrm{pH}$, as the buffer concentration at test range will not vary the physiology of the microbial cell. The term $\left(\mathrm{k}_{\mathrm{E}} \mathrm{C}_{\mathrm{ion}}+1\right)$ is rewritten as $\mathrm{g}$ to simplify the derivation of $\delta \%$ and $\mathrm{C}_{50 \%}$.

$$
\begin{array}{lc}
C=C_{50 \%}+n \delta & \text { Equation.3.41 } \\
x=x_{50 \%}+n \delta_{\%} & \text { Equation.3.42 } \\
f(x)=\left(K_{E} C_{i o n}+1\right)\left(x_{50 \%}+n \delta_{\%}\right)=1 & \text { Equation.3.43 }
\end{array}
$$


The value of $\mathrm{f}(\mathrm{x})$ will be constantly equal to 1 . The unknown constant of $\delta_{\%}, \mathrm{~K}_{\mathrm{E}}$ and $\mathrm{C}_{50 \%}$ will be constant at same $\mathrm{pH}$. $\mathrm{C}_{\text {ion }}$ is the buffer ionic concentration. Data comparison of $2 \mathrm{f}(\mathrm{x})$ at the same $\mathrm{pH}$ in different concentrations will give Equation.3.44. It can be rewritten to Equation.3.45, a relationship between $\mathrm{x}_{50 \%}$ with $\delta \%$. Random selection of two tests at the same $\mathrm{pH}$ and different concentrations will result the same value of $\mathrm{x}_{50 \%}$ and $\delta_{\%}$. Therefore, comparison of two $\mathrm{x}_{50 \%}$ shows the gradient between $\mathrm{x}_{50 \%}$ and $\delta_{\%}$ will be a constant in all concentrations at the same $\mathrm{pH}$. Equation.3.46 is derived with the constant gradient G. $\mathrm{K}_{\mathrm{E}}$ can be calculated thourgh combination of the two different OD test values (Equation.3.46).

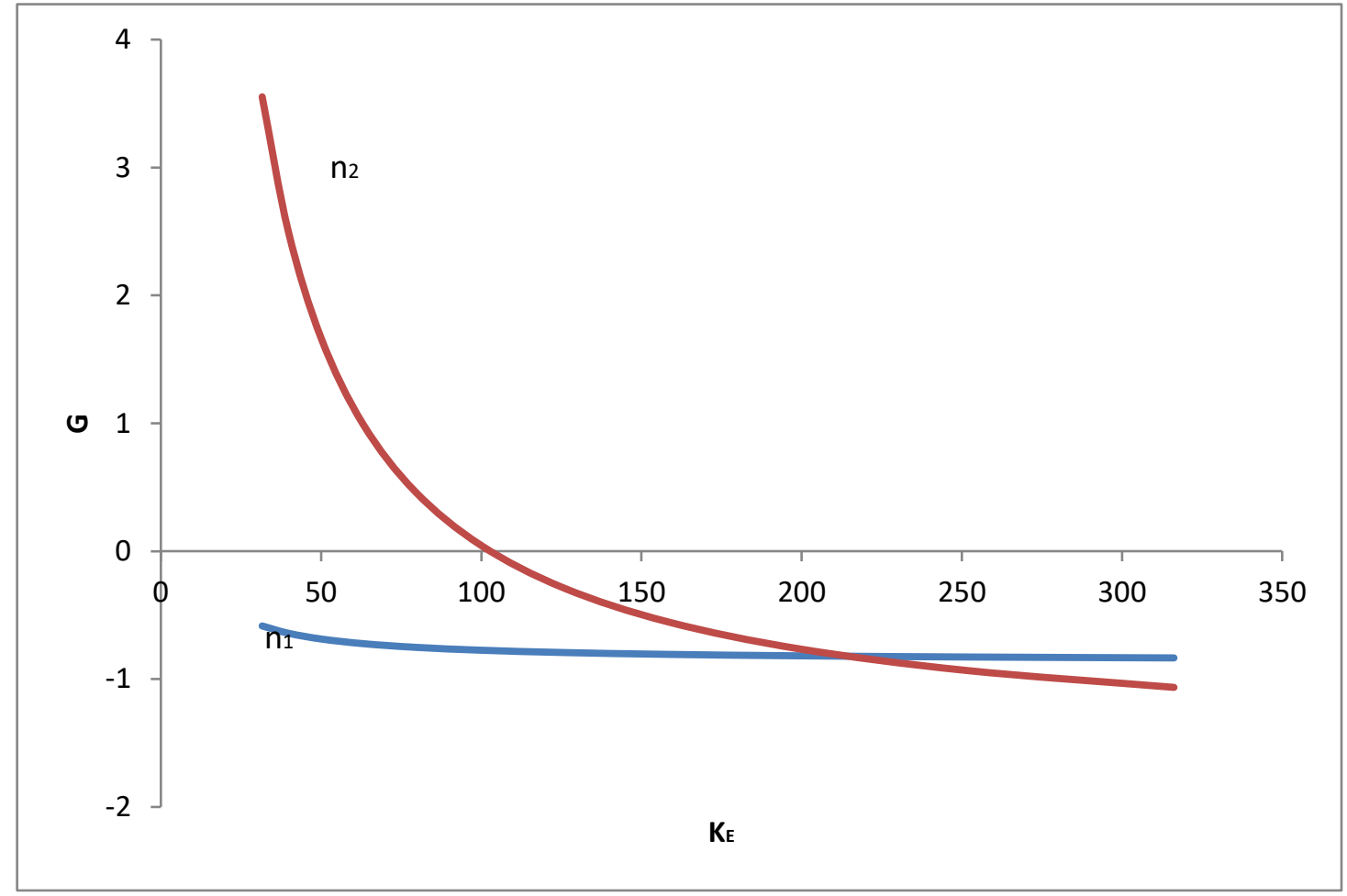

Figure.3. 28. example plot of $\mathrm{G}$ vs $\mathrm{K}_{\mathrm{E}}$ of two randomly n values used.

$$
\begin{aligned}
& g_{1}\left(x_{50 \%}+n_{1} \delta_{\%}\right)=g_{2}\left(x_{50 \%}+n_{2} \delta_{\%}\right) \\
& x_{50 \%}=\frac{\left(g_{2} n_{2}-g_{1} n_{1}\right)}{\left(g_{1}-g_{2}\right)} \delta_{\%}=G \delta_{\%} \\
& \left\{\frac{\left(g_{2} n_{2}-g_{1} n_{1}\right)}{\left(g_{1}-g_{2}\right)}\right\}_{1}=\left\{\frac{\left(g_{2} n_{2}-g_{1} n_{1}\right)}{\left(g_{1}-g_{2}\right)}\right\}_{2}
\end{aligned}
$$

Equation.3.44

Equation.3.45

Equation.3.46

A minimum of 2 groups of 2 OD comparisons with at least partially different values were required in the $\mathrm{K}_{\mathrm{E}}$ estimation. Therefore, $3 \mathrm{OD}$ tests in different buffer concentrations at the same $\mathrm{pH}$ was the minimum requirement of data to establish the 
$\mathrm{K}_{\mathrm{E}}$ estimation operation. A plot of the gradient $\mathrm{G}$ against $\mathrm{K}_{\mathrm{E}}$ is shown in Figure.3.28. It is obvious that there is only one intersection point of the two curves. The $\mathrm{K}_{\mathrm{E}}$ value can be found as the intersection point of the two curves, because it is the unique point of $\mathrm{G}_{1}=\mathrm{G}_{2}$.

The standard deviation was calculated with a combination of Equation.3.40 and Equation.3.46, as $\mathrm{K}_{\mathrm{E}}$ is calculated. Comparison of two groups of testing provided the equation of standard deviation as Equation.3.47. Therefore, the $\mathrm{x}_{50 \%}$ is the only unknown value and it can be simply figured out with the standard deviation, reaction equilibrium constant and Equation.3.45.

$$
\delta_{\%}=\left(\frac{1}{K_{E} C_{1}+1}-\frac{1}{K_{E} C_{2}+1}\right)\left(\frac{1}{n_{1}-n_{2}}\right)
$$

Equation.3.47

\subsection{Statistical Analysis Model and Data Display}

In this study, AFM force spectroscopy is only applied on a limited area of membrane surface or a single cell for each measurement. Compare to streaming potential and Zeta-Sizer measurements, the sample amount is much smaller for the AFM method being developed in this thesis. Area calculation shows the area under test in streaming potential instrument is millions time larger than the testing surface in AFM force spectroscopy. Millions of cells can be measured in one Zeta-Sizer test while only one cell can be studied in one force curve. Thus, considering the heterogeneity of cell surfaces, it is possible that the calculated value from force curves will be different from the mean value measured from streaming potential or Zeta-Sizer.

To solve the problem induced by heterogeneity, more force curves are measured on different positions of membrane or on different cells. Statistical analysis is then used to treat the data for comparison with the results from streaming potential or Zeta-Sizer. The Mann-Whitney U test (MWW) was applied in this thesis based on Gaussian distribution, and used in the comparison of the AFM measured data. The application of statistical calculations, data display and the method of MWW are now 
discussed.

\subsubsection{Statistical Analysis and Data Display Based on Gaussian Distribution}

For both membrane and cell samples, the measured sample surface is limited in a small area. Each measurement required a separation of the colloid probe from the surface and position reset. Based on this experimental feature, each calculated zeta potential or adhesion force can be regarded as discontinuous sample. Theoretically, any differences are caused by heterogeneity of membrane or cell surfaces. Gaussian distribution is used in this study to analyse the FORTRAN calculated data.

$f\left(x \mid \mu, \sigma^{2}\right)=\frac{1}{\sqrt{2 \sigma^{2} \pi}} e^{-\frac{(x-\mu)^{2}}{2 \sigma^{2}}}$

Equation.3.48

$\mu=\frac{\sum x}{n}$

Equation.3.49

$\sigma=\sqrt{\frac{\sum|x-\mu|^{2}}{n}}$

Equation.3.50

Where function $\mathrm{f}$ is the probability distribution, $\mathrm{x}$ is independent variable at different values of testing data, $\mu$ is mean value and $\sigma$ is standard deviation and $n$ is the number of data involved in the mean value and standard deviation calculation. Mean value and standard deviation was required to be calculated before data points fitted in Gaussian distribution, according to its mathematical expression, as shown in Equation.3.48. The mean value was calculated directly as the average value of the whole data as Equation.3.49 and standard deviation was calculated as Equation.3.50. From Equation.3.48, these values revealed from calculated data groups can be used to display the data in a form of Gaussian distribution. 

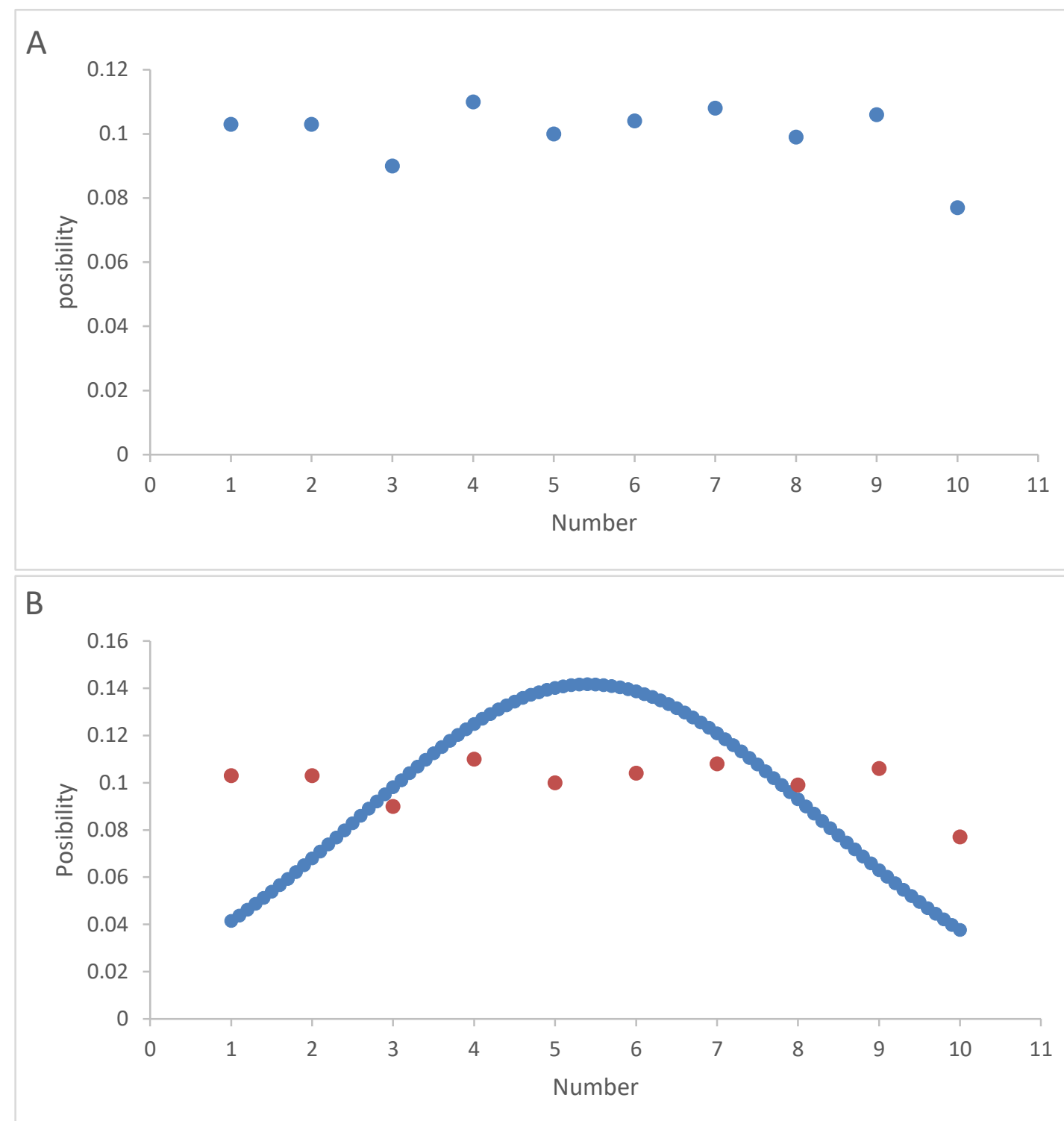

Figure.3. 29. Possibility of real data recorded of 1000 points random integer value distributed at each value (Orange) and its simulated Gaussian distribution (Blue)

An example calculation was used to test the reliability of using Gaussian distribution to illustrate the analysis of the FORTRAN calculated data. Raw data in the test is set as 1000 random integers from 1 to 10 with the RANDI function in MATLAB. The data was plotted in Figure.3.29A, even if the data was set randomly, the program will still try to average the counts of data into each point in the set range of random data selection. The MATLAB data generation is aimed to distribute averagely the 1000 values in the range from 1 to 10 . Different from the data calculated with FORTRAN program, the trend of data distribution is set with this 
generated data. The Gaussian distribution is aimed to generate a distribution that best fits an unknown distribution of the data. The Gaussian distribution curve calculated with the Equations.3.48 - 50 is also calculated and plotted for comparison, as shown in Figure.3.29B. From the raw setting of data generation, a linear relationship that is parallel to the $\mathrm{x}$ axis is the known best fit distribution for the data. Gaussian distribution still shows it can be a suitable distribution for the data, however not as good as linear distribution.

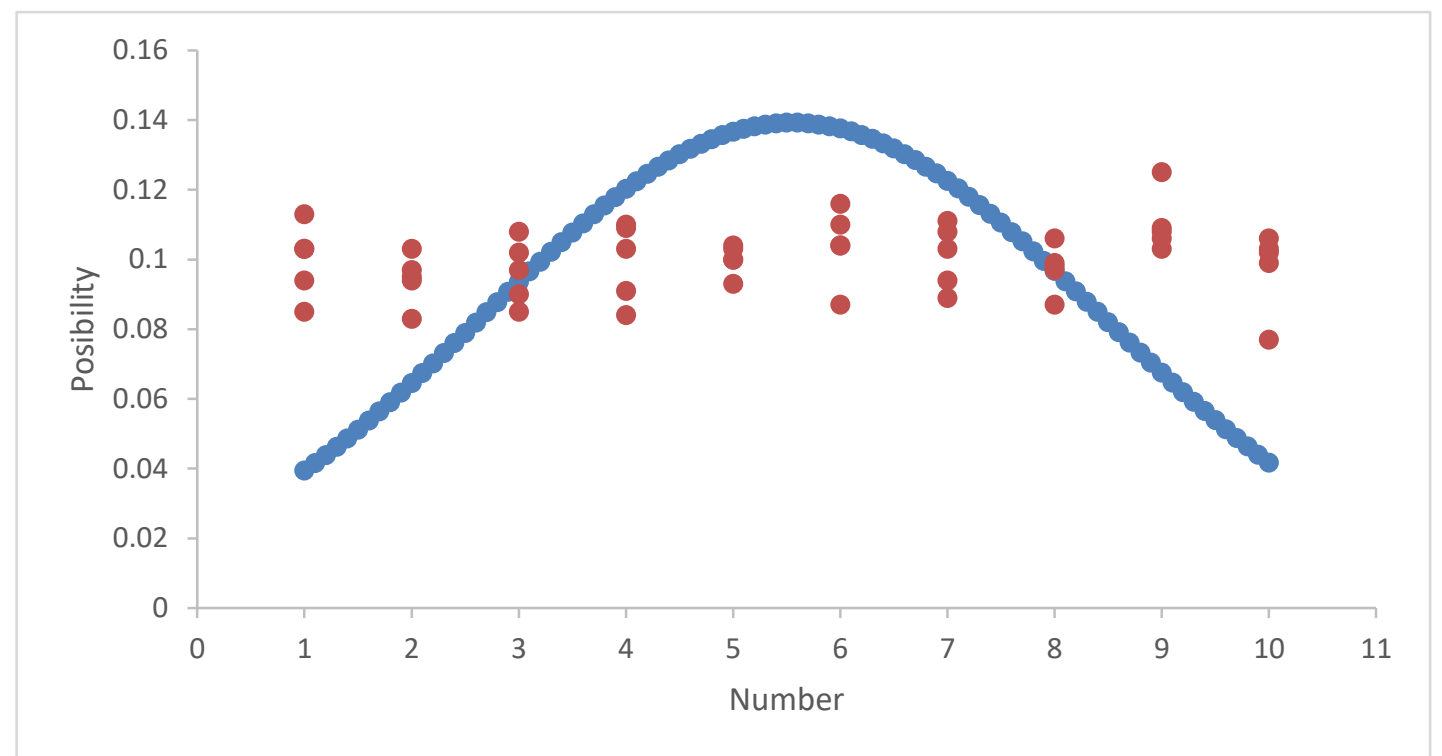

Figure.3. 30. Possibility of real data recorded of 5 groups of 1000 points random integer value distributed at each value (Orange) and its simulated Gaussian distribution (Blue)

To make the data more compliex, 5 arrays of 1000 random integer in range of 1 to 10 was generated with MATLAB, and each array was counted separately. This made the distribution of data becomes more random and deviate from linear distribution. A count of raw data and a calculated Gaussian distribution are plotted in Figure.3.30. Comparing Figure.3.30 and Figure.3.29b, Gaussian distribution becomes more suitable when the linear relationship is weakened in the verification testing. Gaussian distribution is significantly more suitable to represent a more random data. 


\subsubsection{Mann-Whitney U Test}

Mann-Whitney U test (MWW) is a mature model used in data comparison. In this study, the model is used to compare two different groups of AFM force curve calculated data to check if there is a significant difference. This method is used in the study of buffer, STP and Amoxicillin influence on sample surfaces. The technique of MWW is described in this section.

Many calculation tools can establish a MWW calculation and analysis system. To simplify the system construction and display the calculation straightforwardly, Excel was selected in this study as platform of MWW. Before any calculations were applied, two groups of data from the FORTRAN Force-Curve analysis with different conditions were imported into an Excel worksheet. The two groups of data were marked as group 1 and group 2, and each data was marked with the group number for following calculations. The two groups of data were mixed as a single array and set in a ranking with ascending order. Equal values in the ranking were marked with a ranking with the same value that equals to average of the values' rankings, for example, two equal values in number 35 and 36, both number should be marked as 35.5. To achieve this function, the feasible ranking can be recalculated by Equation.3.51 associated with the ranking function in Excel.

$R_{M W W}=R_{\text {ascending }}+\frac{N+1-R_{\text {ascending }}-R_{\text {descending }}}{2}$ Equation.3.51

Where $\mathrm{R}_{\mathrm{MWw}}$ is the ranking used in MWW, $\mathrm{R}_{\text {ascending }}$ is ranking in ascending order, $\mathrm{N}$ is total number of data involved in ranking and $\mathrm{R}_{\text {descending }}$ is ranking in descending order. With the Excel function of COUNTIF, how many data from each group is counted, and the counts is marked as $\mathrm{N}_{1}$ for group 1 and $\mathrm{N}_{2}$ for group 2. The Excel function SUMIF was used to calculate the sum of the ranking for each group and marked as $\mathrm{R}_{1}$ for group 1 and $\mathrm{R}_{2}$ for group 2 , then the ranks for both groups were summed. If the sum of the totals equals to $R_{1}$ plus $R_{2}$, it means there is no error in ranking generation and the value of sum of total is marked as $U_{\text {total. }} U_{1}$ and $U_{2}$ are then calculated with the $\mathrm{N}$ and $\mathrm{R}$ values from Equation.3.52 \& 53. The smaller value from $U_{1}$ and $U_{2}$ are marked as the value $U$. A value of $U_{a}$ is calculated as 
Equation.3.54 for comparison.

$U_{1}=N_{1} N_{2}+\frac{\left(N_{1}+1\right) N_{1}}{2}-R_{1}$

Equation.3.52

$U_{2}=N_{1} N_{2}+\frac{\left(N_{2}+1\right) N_{2}}{2}-R_{2}$

Equation.3.53

$U_{a}=\frac{U_{1} U_{2}}{2}$

Equation.3.54

The comparison is made between $U$ and $U_{a}$, if $U$ is larger than $U_{a}$ it means there is no significant difference between the two groups of data and a significant difference between the two groups of data can be verified if $U$ is smaller than $U_{a}$.

\subsection{Conclusion}

In this study, a series of calculations are engaged to assist the data analysis. Development of these calculation models have been discussed in this chapter.

A FORTRAN program calculation system was developed based on DLVO theory that could deconvolve (extract) electrostatic interaction from AFM force curves and calculate surface zeta potential from a force curve. Adhesion analysis from force curves was also achieved with numerical methods applied. The FORTRAN program was widely used through the whole study to guide the analysis of interaction information from AFM force curves, with the results presented and discussed in Chapter IV and V. Within this chapter, the model and FORTRAN program was also calibrated and validated with DLVO theories and compared with previous research. Compared with a linearized simulation, the developed model was more accurate. Verification also showed that influence of Van der Waals force is negligible and could be arguably be ignored with an acceptance of minimal error. Errors will also be induced from calculation of surface charge and surface geometry integration as well. The overall influence of van der Waals forces, surface charge and geometry integration is controlled to less than $4.08 \%$, which is not an significant level (less than $5 \%)$.

A hydrophobicity analysis model was also developed based on solvent 
partitioning. This is used by the thesis to assess the influence of hydrophobicity within the cell study (Chapter V). This model uses a Gaussian distribution to provide a more detailed analysis of hydrophobicity, and can also be used in the interpretation of the chemical properties of cell surfaces as well. This represents a novel approach with no previous research examples for comparison beyond simple solvent partitioning to indicate degree of microbial cell hydrophobicity.

The use of the statistical model of MWW is described in this chapter. A data display model that is based on Gaussian distribution was also introduced. These two methods are frequently used in Chapter 4 and 5 for the data comparison and display of zeta potential, adhesion analysis and hydrophobicity analysis. 


\section{Chapter IV AFM Force Analysis of Particle Interaction with Membrane Surface}

\subsection{Introduction}

Membrane surface interactions are important in filtration processes, as seen in the review in section.1.2.2. Surface interactions on Cyclopore membranes (MF) and DK membranes (NF) are studied in this chapter. From the calculation model developed based on the revision on the DLVO theory (section.1.2.1) and atomic force microscopy (AFM) (section.1.3), zeta potential and adhesion on membrane surfaces were calculated in this chapter and the calculated results were analysed with statistical analysis models0, such as Mann-Whitney U test (MWW) (section.3.4.2).

\subsection{Methods}

\subsubsection{Surface Analysis of Membrane Surfaces}

Colloid particles used in the AFM force measurements were silica beads of sizes $5 \mu \mathrm{m}$ and $35.5 \mu \mathrm{m}$. Zeta potentials of the silica beads were measured with Zeta-Sizer, as was introduced in section.2.2.3. Specific gravity of silica beads is 2.54 (Polysciences Inc., 2013); it is a heavy material compared to water. Zeta potentials were measured immediately after the silica beads were suspended in buffers. Surface charges of silica beads were calculated in the FORTRAN program (Appendix.2) from the zeta potentials measured and were regarded as constant at the same buffer $\mathrm{pH}$ and concentration.

\subsubsection{AFM Measurements of Membrane Surfaces}

Park XE-100 AFM was used for the AFM measurements on DK and Cyclopore membrane surfaces (Fei \& Brock, 2013). Liquid cell for Park AFM was used in the measurements to avoid vacuum effects and maintain the measuring conditions as bio-confident (Wright \& Revenko, 2004). Zeta-Sizer measurements on silica beads 
were used in the calculation of colloid probe surface charges. Scanning scale $(9.47 \mu \mathrm{m}$ $\mathrm{X} 9.47 \mu \mathrm{m}$ ) was used in the AFM contact mode imaging. The scanned image was directly used as an auxiliary surface topography for force measurements.

The AFM imaging setup was introduced in section.2.2.5.3. Membrane samples were prepared according to the method described in section.2.2.4.1. AFM force measurements were applied after imaging. AFM topography image analysis software was used to analyse membrane surface curvatures (section.3.2.5.2). All measurements were operated in liquid environments that were created by the AFM liquid cell.

\subsubsection{Application of Computational Analysis System for Membrane Surface Force Properties Analysis}

The analysis of force curves measured on membrane surfaces were based on the FORTRAN code that developed from the model developments as shown in section.3.2.3. Adhesions were studied with the method that was introduced in section.3.2.4. The whole calculation model was developed as the structure that was described in section.2.3.1. Zeta potential mapping was applied based on the interest of aims and objectives (section.1.5). It was achieved with the zeta potential calculation that adapts to the AFM force mapping mode.

These tested and calculated data was analysed statistically. Mann-Whitney U test (MWW) was applied in data comparison (section.3.4.2). Data display system was described in detail in section.3.4.1.

\subsection{Membrane Surface Zeta Potential Calculation Using Atomic Force Spectroscopy}

Force curves were measured at different buffer concentrations and $\mathrm{pH}$. The zeta potentials, which were calculated from the force curves, assumed that ideal flat surface with membrane surface curvature effect was applied, as was discussed in section.3.2.5.1. Zeta potential distributions across the surface are analysed statistically 
and discussed in this section.

\subsubsection{AFM Force Curves Measured in Aqueous Environment}

Force curves were measured by using colloid probes. Force curves that were measured on membrane surfaces were shown in Figure.4.1 for Cyclopore membranes and Figure.4.2 for DK membranes at different $\mathrm{pH}$ and same ionic strength. The force curves measured in different buffer ionic strengths were presented in Figure.4.3 for Cyclopore membranes and Figure.4.4 for DK membranes. Adhesion forces were observed in retraction force curves. Fluctuation of adhesions (Figure.4.2c) and large adhesion forces (Figure.4.1b) were obvious in some force curves, and some other force curves were applied that showed no adhesion force (Figure.4.1a).

Comparison of different force curves showed that the adhesion forces applied on membrane surfaces were more significant compared to other interactions. However, it appears to be randomly applied when compared to the retraction force curves at different $\mathrm{pH}$ and ionic strengths (Figure.4.1 - 4.4). As was described in section.3.2.4, the adhesion forces applied on both membrane surfaces were counted and analysed in order to identify the influences that occurred from $\mathrm{pH}$ and ionic strength influences, which was studied and described in the section.4.5.1.

From Figure.4.1a and Figure.4.1b, repulsive and attractive forces were found from approaching force curves. The force was a convolution of different interactions, including van der Waals forces, forces induced by surface roughness, chemical bonding forces and electrostatic forces. It is difficult to identify the influence of electrostatics influences from the forces measured on probes, which was concealed in the convolution force as a part of the interaction applied before surface contact. Therefore, the calculation model that developed in section.3.2 was used to deconvolve the electrostatic forces from the measured force curves. With the calculated zeta potentials, buffer influences were analysed as is stated in the following section (section.4.3.2) and then, studied with mapping mode for its topographical distribution (section.4.4). 

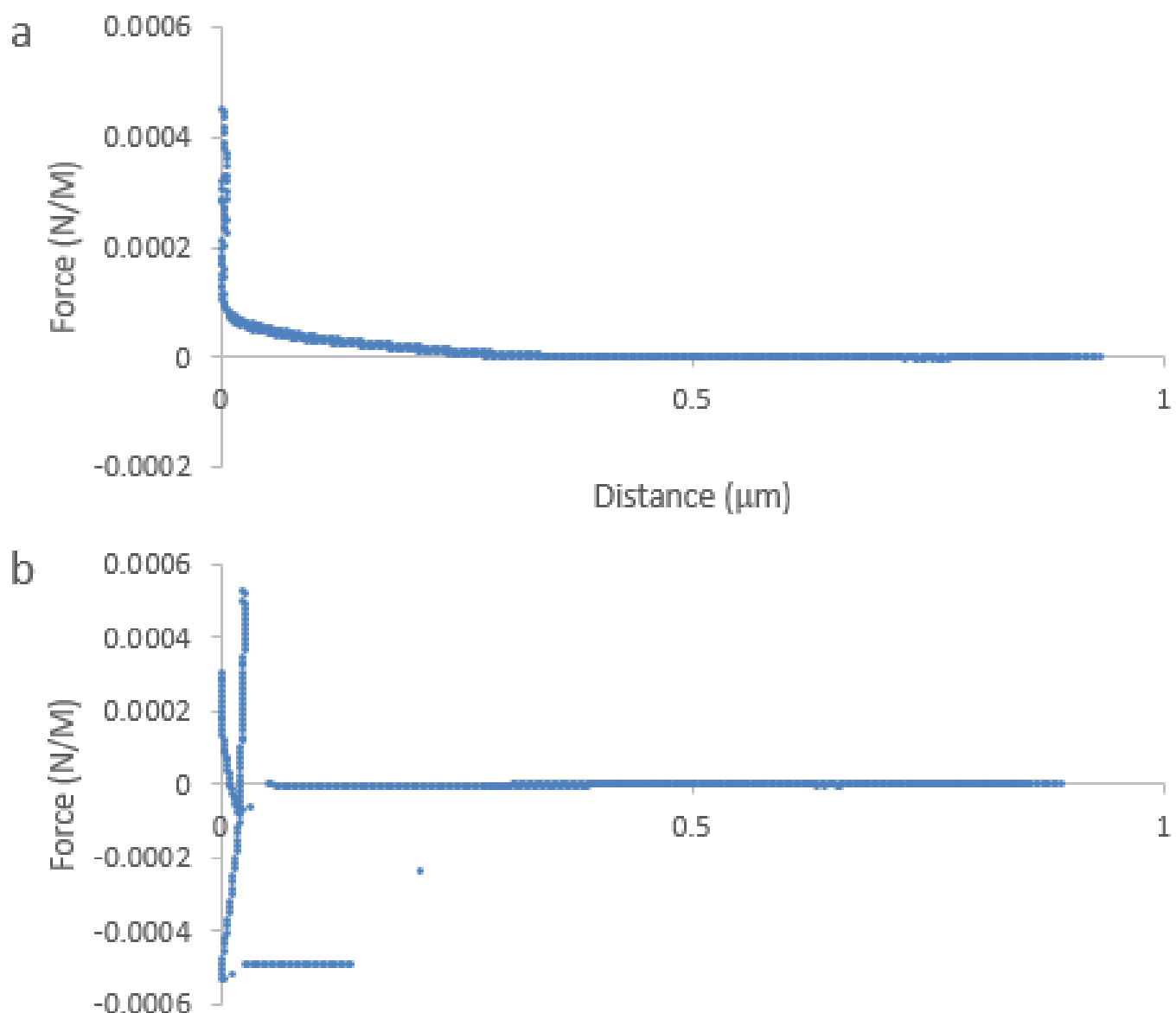

Distance $(\mu \mathrm{m})$

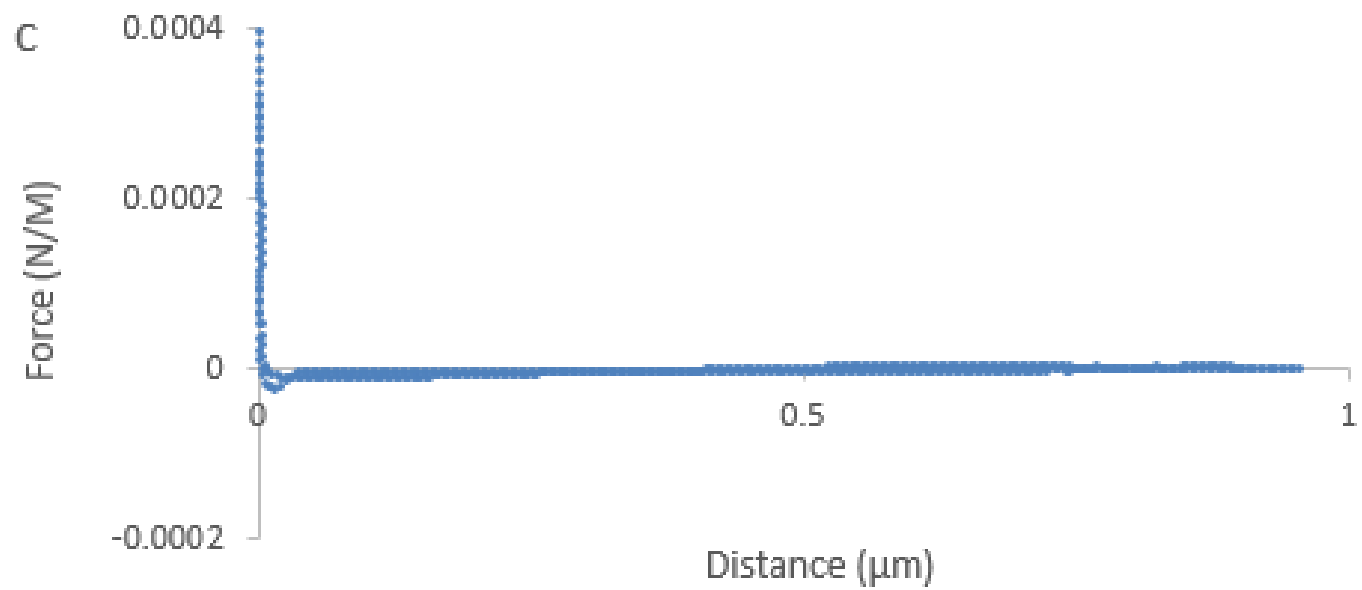

Figure.4. 1. Example of force curves measured on cyclopore membrane surface in different buffer $\mathrm{pH}$ conditions and constant ionic strength $(0.1 \mathrm{M} \mathrm{NaCl}$ solution), a in $\mathrm{pH} 5, \mathrm{~b}$ in $\mathrm{pH} 7$ and $\mathrm{c}$ in $\mathrm{pH} 9$ buffer. 

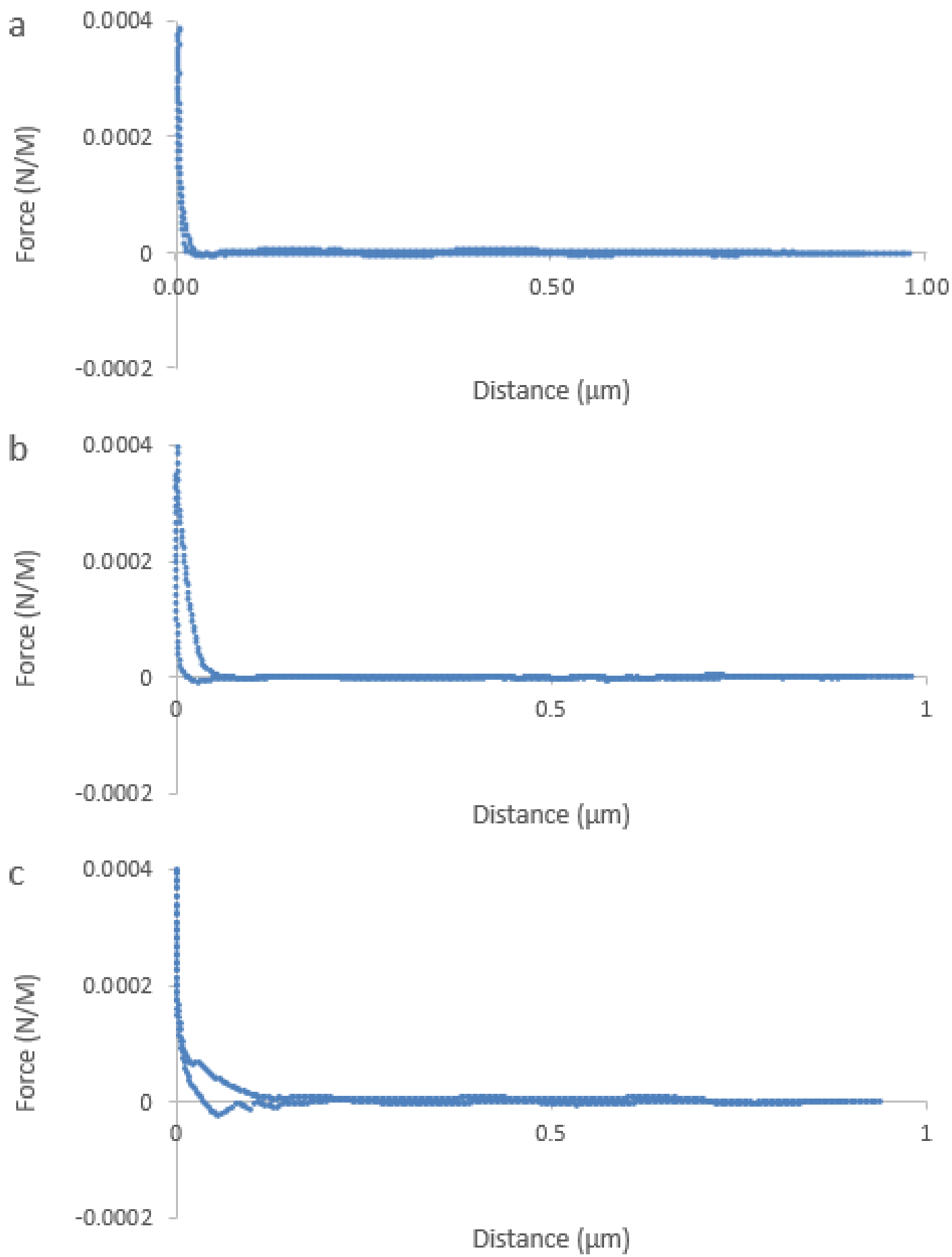

Figure.4. 2. Example of force curves measured on DK membrane surface in different buffer conditions and constant ionic strength $(0.1 \mathrm{M} \mathrm{NaCl}$ solution), $\mathrm{a}$ in $\mathrm{pH} 5, \mathrm{~b}$ in $\mathrm{pH} 7$ and $\mathrm{c}$ in $\mathrm{pH} 9$ buffer. 


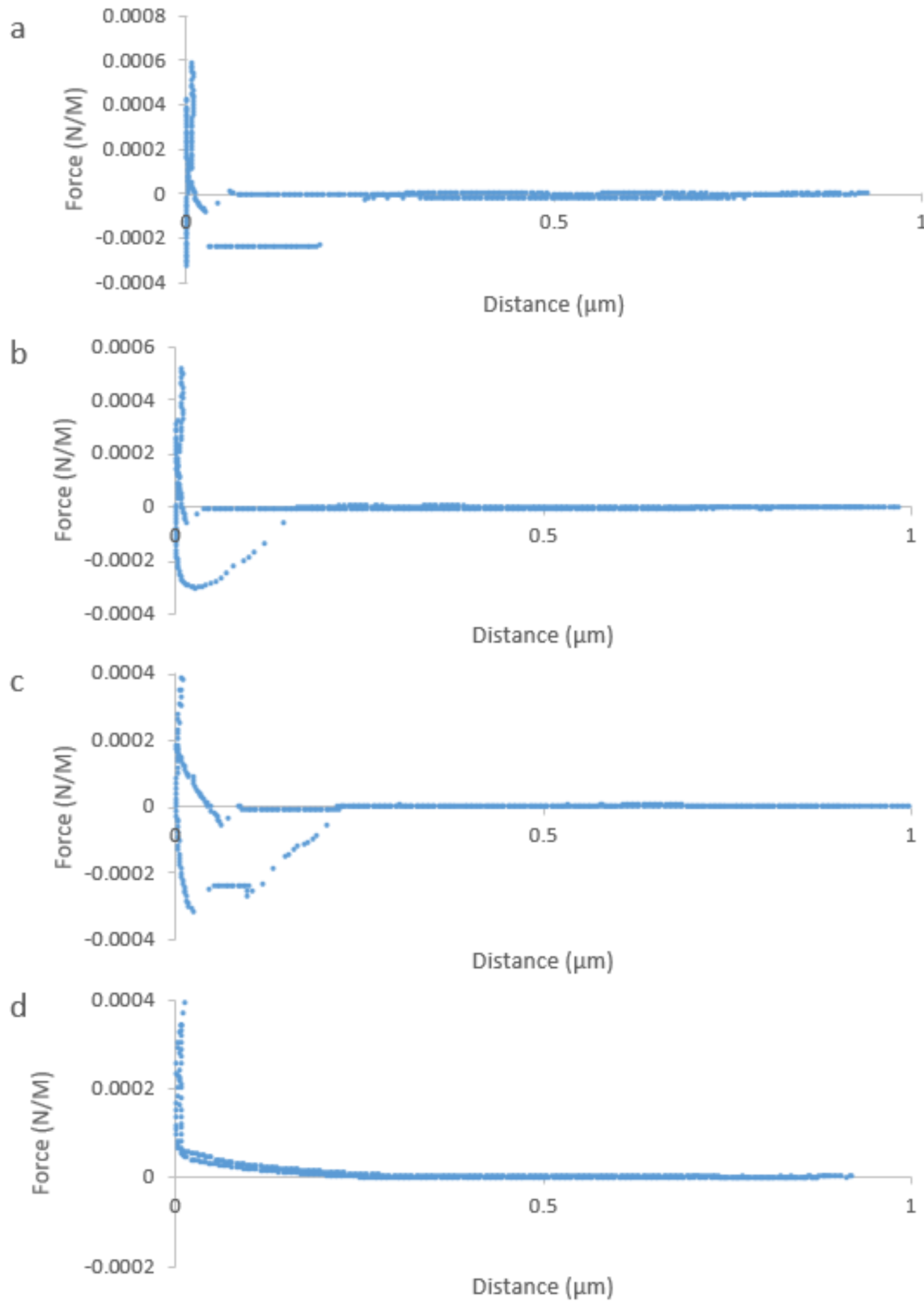

Figure.4. 3. Example of force curves measured on cyclopore membrane surface in different buffer ionic strength and constant $\mathrm{pH}(\mathrm{pH}$ ), a in DI water, b in $0.001 \mathrm{M}$ $\mathrm{NaCl}$ solution, $\mathrm{c}$ in $0.01 \mathrm{M} \mathrm{NaCl}$ solution and $\mathrm{d}$ in $0.1 \mathrm{M} \mathrm{NaCl}$ solution. 


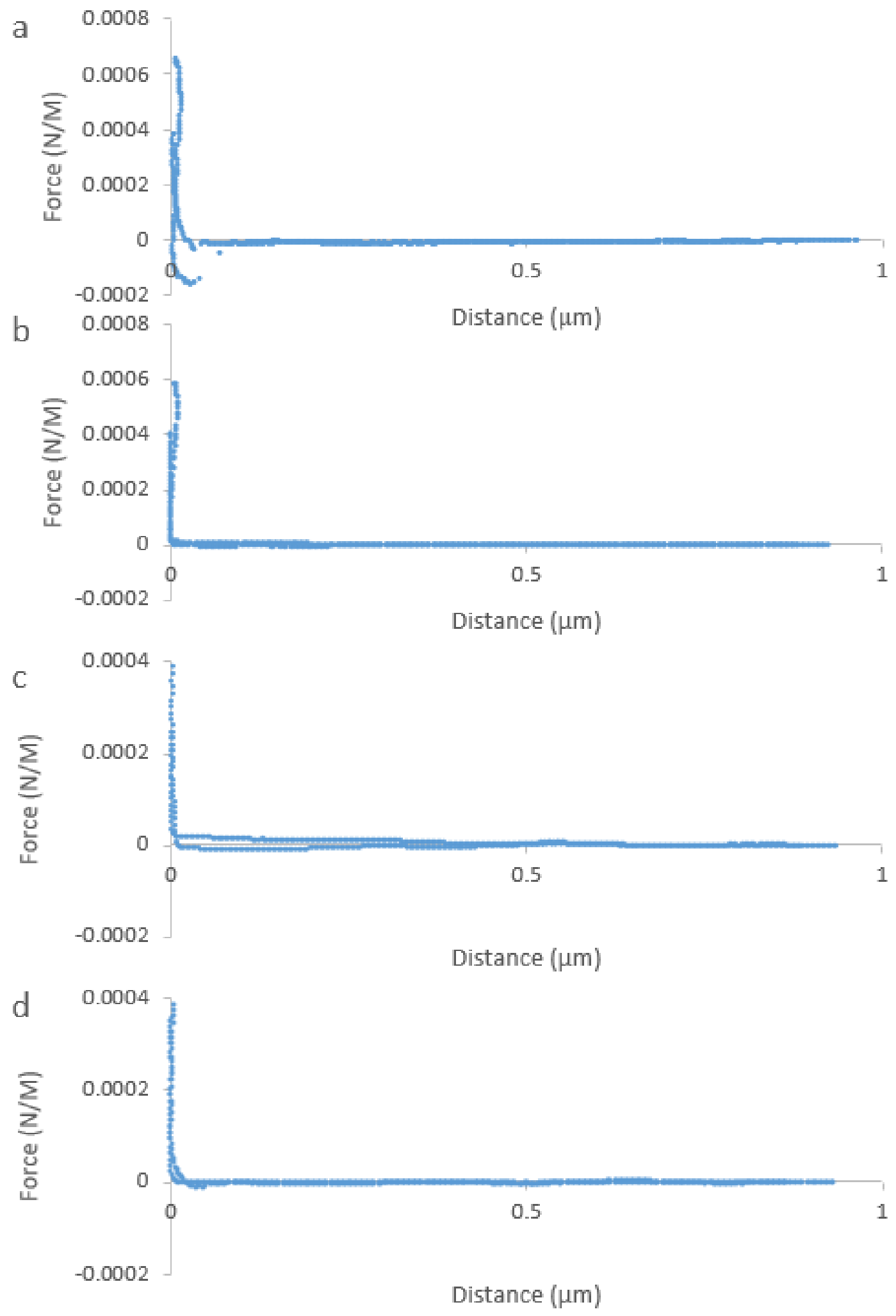

Figure.4. 4. Example of force curves measured on DK membrane surface in different buffer ionic strength and constant $\mathrm{pH}(\mathrm{pH} 5)$, a in DI water, $b$ in $0.001 \mathrm{M} \mathrm{NaCl}$ solution, $\mathrm{c}$ in $0.01 \mathrm{M} \mathrm{NaCl}$ solution and $\mathrm{d}$ in $0.1 \mathrm{M} \mathrm{NaCl}$ solution. 


\subsubsection{Zeta Potential Calculation on Membrane Surfaces with AFM Force Curves}

Zeta potentials were calculated from the force curves that were measured on the DK and Cyclopore membrane surfaces with surface charge densities of silica colloids that were calculated from Zeta-Sizer, which measured the zeta potential (Figure.4.5) in different buffers. 15 to 50 force curves that were measured in the same buffer were applied in the zeta potential calculation, which were then analysed statistically and examined the buffer influences on membrane surface electrostatics.

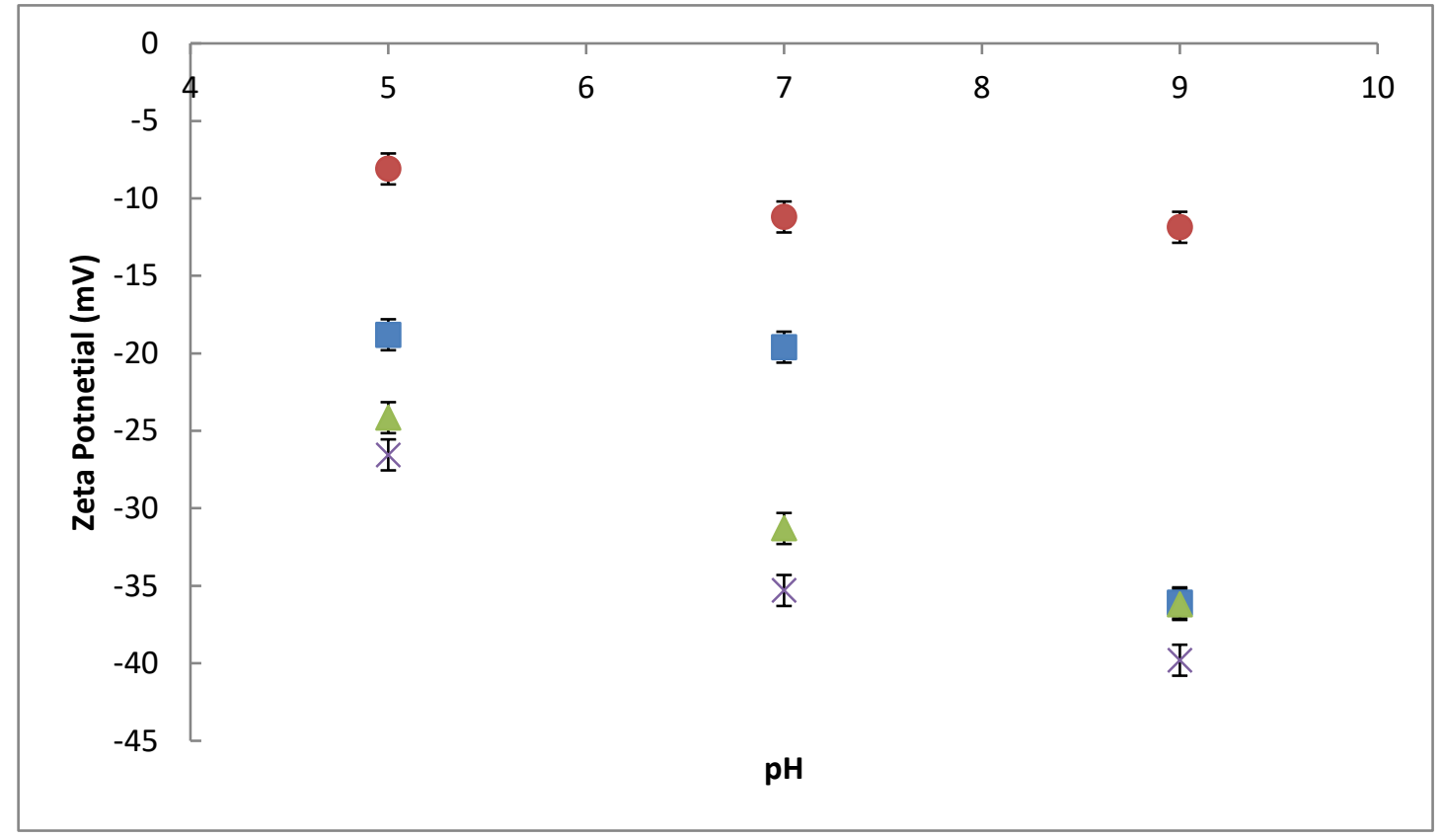

Figure.4. 5. Zeta potential of silica beads measured with zeta-sizer in different $\mathrm{pH}$ and ionic strengths. Measurements are all in $\mathrm{NaCl}$ solutions or DI water. Purple cross is in DI water, read dot in $0.1 \mathrm{M}$, blue square in $0.01 \mathrm{M}$ and green triangle in $0.001 \mathrm{M}$ of $\mathrm{NaCl}$ solutions.

The measured zeta potentials were compared with MWW and significant differences were shown between any two sets of zeta potentials that were measured at different buffer conditions. Different from that found in previous studies, such as Al-Amoudi et al.'s study in 2007 (Al-Amoudi et al., 2007), the zeta potentials calculated from force curves were randomly influenced by buffer $\mathrm{pH}$ and ionic strengths (Figure.4.6). Similar to previous studies, for example, Oatley et al.'s study in 2013 (Oatley et al., 2013), zeta potentials were mostly found to be negative from pH5 to pH9. 

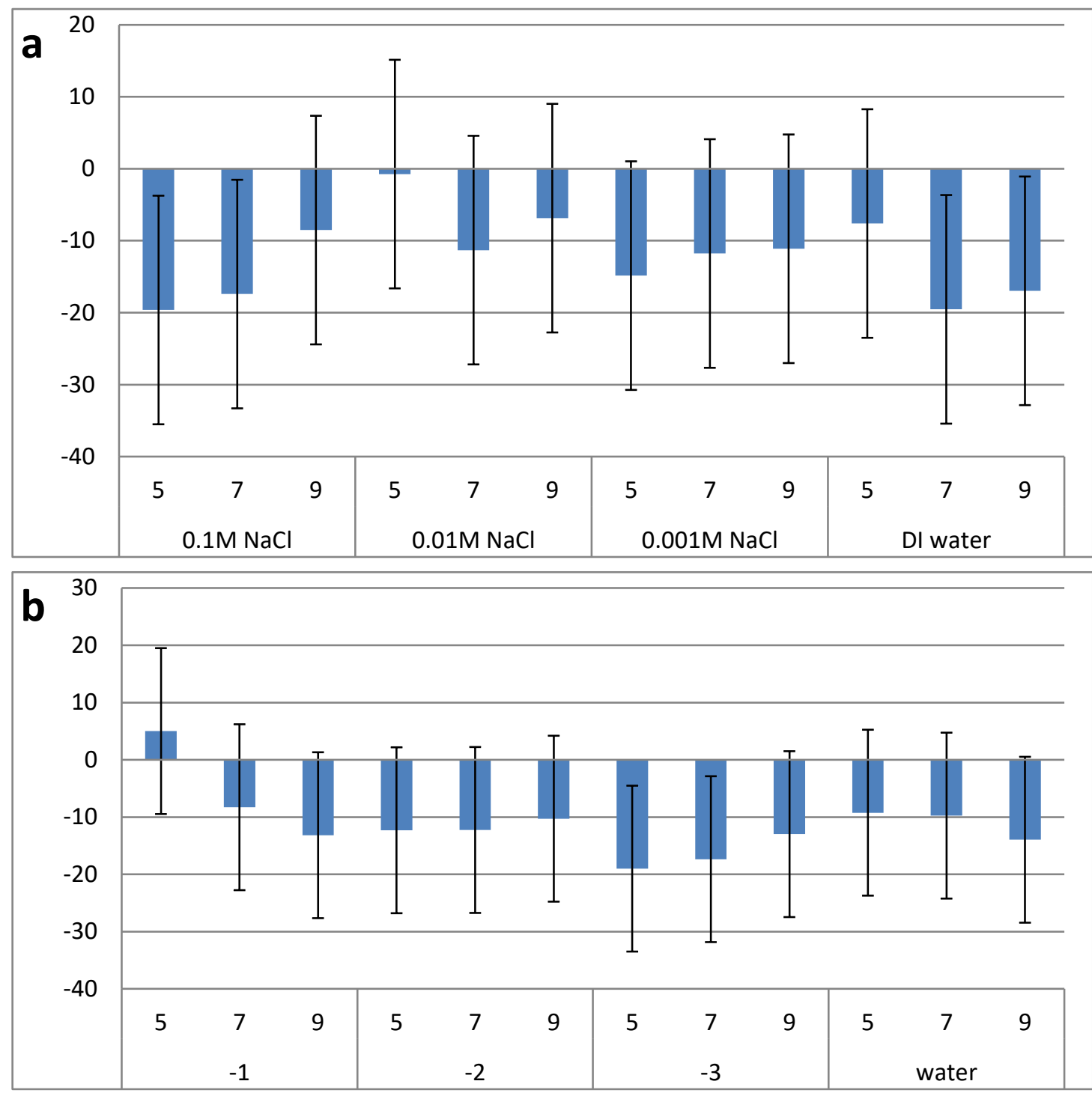

Figure.4. 6. Average zeta potential of cyclopore membrane surface (a) and DK membrane surface (b). Both plots are with error estimations. Unit is in $\mathrm{mV}$ for both plots.

Topography imaging of membrane surface also presents the roughness of both membrane surfaces, which are significant in this study. As discussed in section.1.2.2, roughness is induced in membrane fabrication and potentially brings in heterogeneity influences that is applied on surface interactions. The calculation of zeta potentials from AFM force curves were theoretically verified with negligible impact from other interactions and factors (section.3.2.6). Thus, standard deviations of zeta potential on DK and Cyclopore membranes were calculated and presented in Figure.4.6a \& b as error bars, which is the range of most zeta potentials located in $( \pm 1$ standard 
deviation). Standard deviations were about $\pm 15 \mathrm{mV}$ for both membranes, which is a large distribution range compared to the average zeta potentials calculated. Considering the areas that were measured by AFM force measurements (cross-sectional area of colloid probes), it is reasonable that large heterogeneity was found on the surfaces.

The measured zeta potentials were then compared with those measured using another mature method to verify its reliability. Thus, a review of membrane zeta potential measured with streaming potential was applied and compared with the AFM calculated results in the following section.

\subsubsection{Review of Membrane Surface Zeta Potential with Previous Research Based on Streaming potential and Comparison with AFM Zeta Potential Estimation}

Streaming potentials on the DK membrane surfaces were used to illustrate the average zeta potential at different buffers. It was compared with the zeta potentials calculated with the AFM force curves. From the review of previous researches on other membrane technologies, membrane type, ions in buffer and $\mathrm{pH}$ were all found to be influential to the streaming potential results (Bellona \& Drewes, 2005; Shim et al., 2002; Vrijenhoek et al., 2001). Buffer influences were not discussed and set as a constant concentration in some membrane studies (Al-Amoudi et al, 2007; Deshrnukh \& Childress, 2001). However, many other studies showed that the buffer concentrations provided a significant influence to the zeta potential measured from streaming potential (Hurwitz et al., 2010; Zhao et al., 2005).

Detailed results of streaming potentials measured under the stated buffer condition were shown and discussed in the study of Oatley et al. in 2013 (Oatley et al., 2013). Referring to the research from Oatley et al., average DK membrane zeta potentials measured with streaming potential were shown in Figure.4.7 a in $0.01 \mathrm{M}$ of ionic buffer of $\mathrm{KCl}, \mathrm{NaCl}, \mathrm{Na}_{2} \mathrm{SO}_{4}$ and $\mathrm{MgSO}_{4}$ (Oatley et al., 2013). Zeta potentials became more negative with increase in $\mathrm{pH}$. The estimation of zeta potentials with AFM were in buffer $\mathrm{pH}$ of 5,7 and $9(-12 \mathrm{mV}$ at $\mathrm{pH} 5,-12 \mathrm{mV}$ at $\mathrm{pH} 7$ and $-10 \mathrm{mV}$ at $\mathrm{pH} 9$ in 0.01 
$\mathrm{M} \mathrm{NaCl}$ buffers), while, that was found to be $-10 \mathrm{mV}$ in $\mathrm{pH} 5,-12 \mathrm{mV}$ in $\mathrm{pH} 7$ and -14 $\mathrm{mV}$ in pH9 (Thomas et al., 2016). The measured sample areas of AFM are significantly smaller than the streaming potential measured areas. AFM estimation results would reflect more on the heterogeneity, but the measured areas were not as large as the streaming potential to provide an overview of the average zeta potential on a large surface area of membrane surfaces. The differences of average zeta potentials measured from streaming potentials and force curves calculations were large. However, the differences found were less than $\pm 5 \mathrm{mV}$ at all buffers. Thus, streaming potential results were in the concentrated region of the Gaussian distribution that analysed force curves calculated by zeta potentials, which is not significant when compared to the standard deviation $(<0.33$ standard deviation). The calculated results of the force curves were regarded as a reliable measurement on zeta potential distribution, while the accuracy of average zeta potentials was limited by the amount of force curves applied in the calculation. Thus, zeta potential heterogeneity was analysed from the calculated results of the force curves, even though it would not provide average zeta potentials as accurate as streaming potential because of the small measuring area of colloid probes and small amount of force curves qualified for calculation.

Buffer $\mathrm{pH}$ and ionic strength influences on zeta potentials were studied by Thomas et al. (Thomas et al., 2016). A result plot, as shown in Figure.4.7 b, directly presented the influences of ionic strengths and $\mathrm{pH}$. In $0.001 \mathrm{M} \mathrm{NaCl}$ buffers, the calculated results of the force curve had average zeta potentials of $-18 \mathrm{mV}(\mathrm{pH} 5),-16$ $\mathrm{mV}(\mathrm{pH} 7)$ and $-13 \mathrm{mV}(\mathrm{pH} 9)$ with a standard deviation of around $\pm 15 \mathrm{mV}$. However, the quoted streaming potential results from Figure. $4.7 \mathrm{~b}$ showed a result of $-14 \mathrm{mV}$ in $\mathrm{pH} 5,-17 \mathrm{mV}$ in $\mathrm{pH} 7$ and $-19 \mathrm{mV}$ in $\mathrm{pH} 9 . \mathrm{A} \pm 6 \mathrm{mV}$ difference was found, which is not significant as well $(<0.4$ standard deviation). 

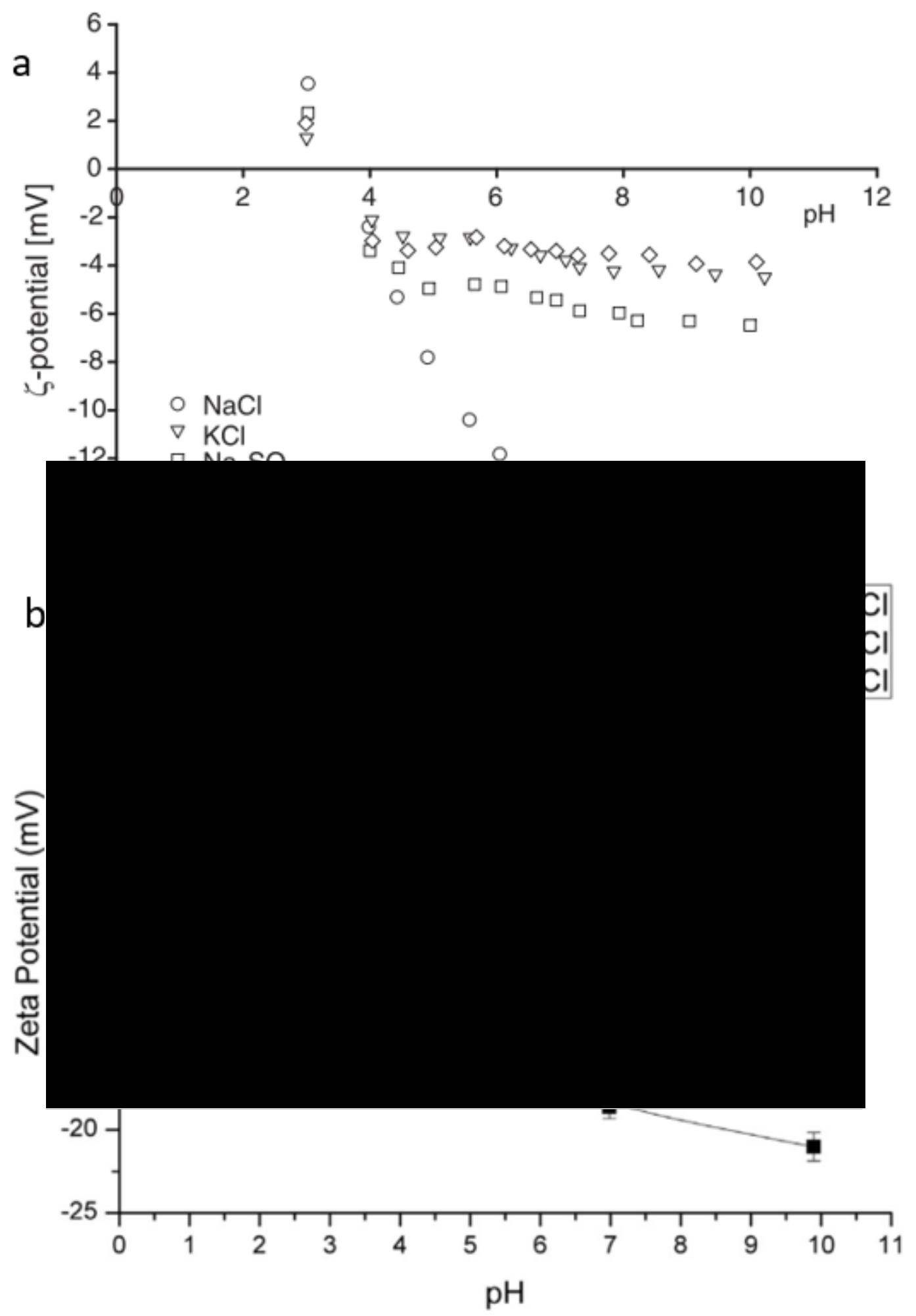

Figure.4. 7. $\mathrm{a}$ is the average zeta potential measured with streaming potential from $\mathrm{pH}$ 3 to 11, cited from (Oatley et al., 2013); b is the streaming potential zeta potential results between $\mathrm{pH}$ of 4 and $10, \mathrm{Y}$ axis as the zeta potential in $\mathrm{mV}$ and $\mathrm{X}$ axis is $\mathrm{pH}$ for both plots, cited from (Thomas et al., 2016).

Streaming potential measurements were also used on DK membranes in 
$0.001 \mathrm{M}, 0.01 \mathrm{M}$ and $0.1 \mathrm{M} \mathrm{NaCl}$ solutions at different $\mathrm{pH}$ by Oatley-Radcliffe et al. and the results were showed in their publication in 2017, which are cited and shown in Figure.4.8 (Oatley-Radcliffe, Aljohani, Williams, \& Hilal, 2017). The streaming potentials of $\mathrm{DK}$ membrane were found to be $-3 \mathrm{mV}$ at $\mathrm{pH} 5,-5 \mathrm{mV}$ at $\mathrm{pH} 7$ and were predicted to be $-7 \mathrm{mV}$ at $\mathrm{pH} 9$ in $0.1 \mathrm{M} \mathrm{NaCl}$ buffers, which is different from the force curves calculated results at pH5 $(+3 \mathrm{mV}), \mathrm{pH} 7(-8 \mathrm{mV})$ and $\mathrm{pH} 9(-12 \mathrm{mV})$. Considering the distribution, the differences $( \pm 6 \mathrm{mV})$ were regarded as the influences of standard deviation.

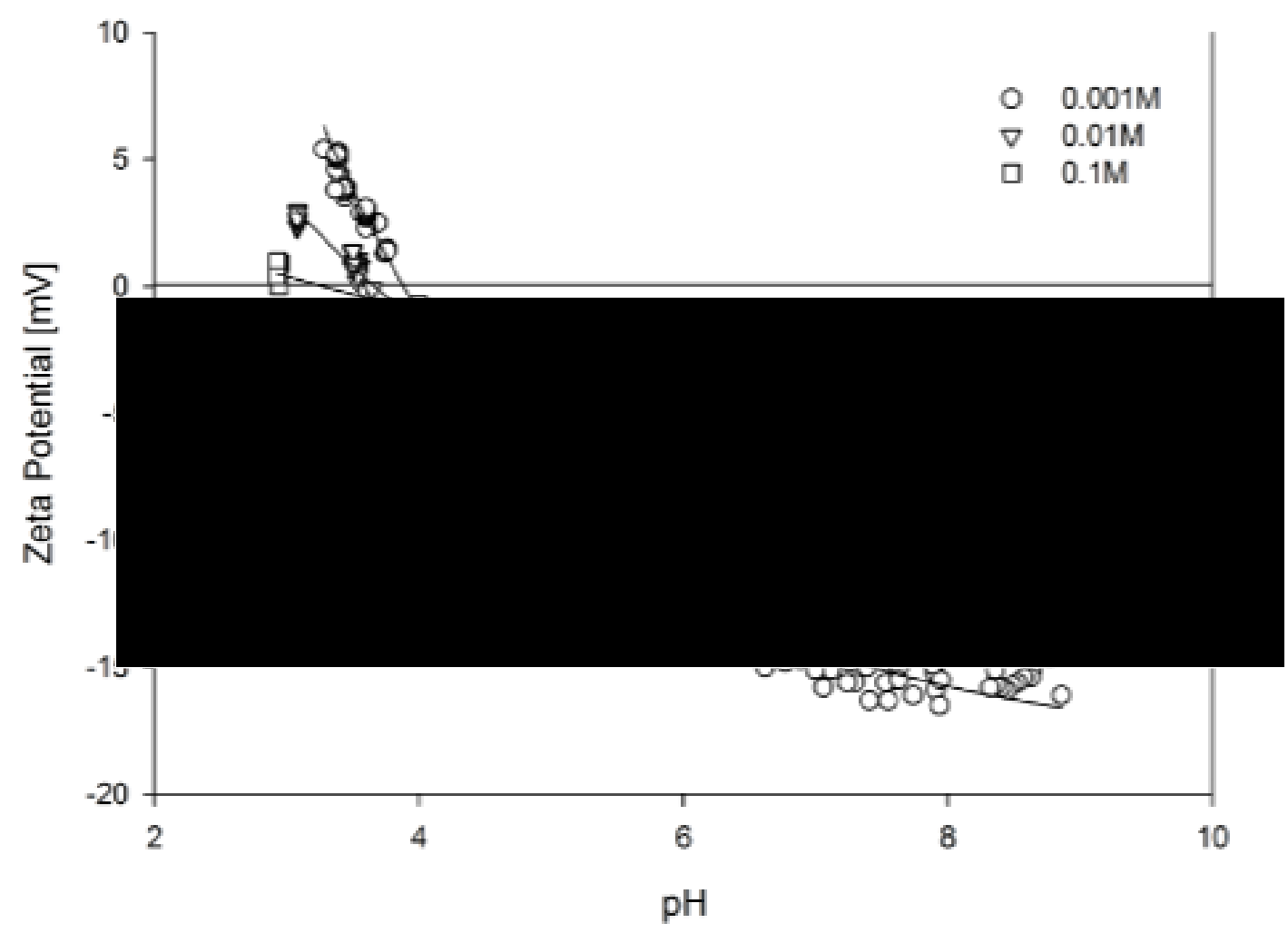

Figure.4. 8. Zeta potential from tangential streaming potential measurements for the Desal DK nanofiltration membrane with $\mathrm{NaCl}$ as the electrolyte, cited from (Oatley-Radcliffe et al., 2017).

Zeta potential heterogeneity on DK membrane surfaces were also found from Figure.4.8, as some of the measured zeta potential were found to be different in the same ionic strength and $\mathrm{pH}$. It is reasonable that electrostatic heterogeneity will become more significant on smaller scale of measuring areas. Therefore, the large standard deviation becomes reasonable at the measuring scale of AFM force 
measurements. Standard deviation calculated with AFM estimation is a reliable illustration of the surface heterogeneity and the distribution is improved with more force curves employed in the calculations. The calculations of the force curves will provide a new function of monitoring the membrane surface electrostatics heterogeneity and calculating the average zeta potentials.

\subsection{Zeta Potential Surface Distribution across a Membrane and the Influence of the Aqueous Environment}

\subsubsection{Zeta Potential Distribution Analysis with AFM Force Mapping}

Zeta potential on membrane surfaces were found to be heterogeneous, as shown in section.4.3. AFM force curve analysis measures a more accurate zeta potential at small scale. Potentially, it can be developed to the zeta potential mapping from the analysis of force curves calculated by zeta potential in conjunction to the AFM force mapping mode. Zeta potentials were measured with measuring positions located at $4 \mathrm{x}$ 4 matrix segmented membrane surfaces.

Zeta potential surface distribution was calculated and shown in Figure.4.9a \& b on $9.47 \mu \mathrm{m}$ x $9.47 \mu \mathrm{m}$ surfaces for both DK and Cyclopore membranes. Zeta potentials of points that were measured on mapping surface were found to be significantly different. Mapping shows the heterogeneity more significantly, and this can be used in buffer influence as well, which is described in the following section (section.4.4.2). Higher resolution of mapping was achieved with larger matrix applied, and this was introduced in section.4.4.3. 

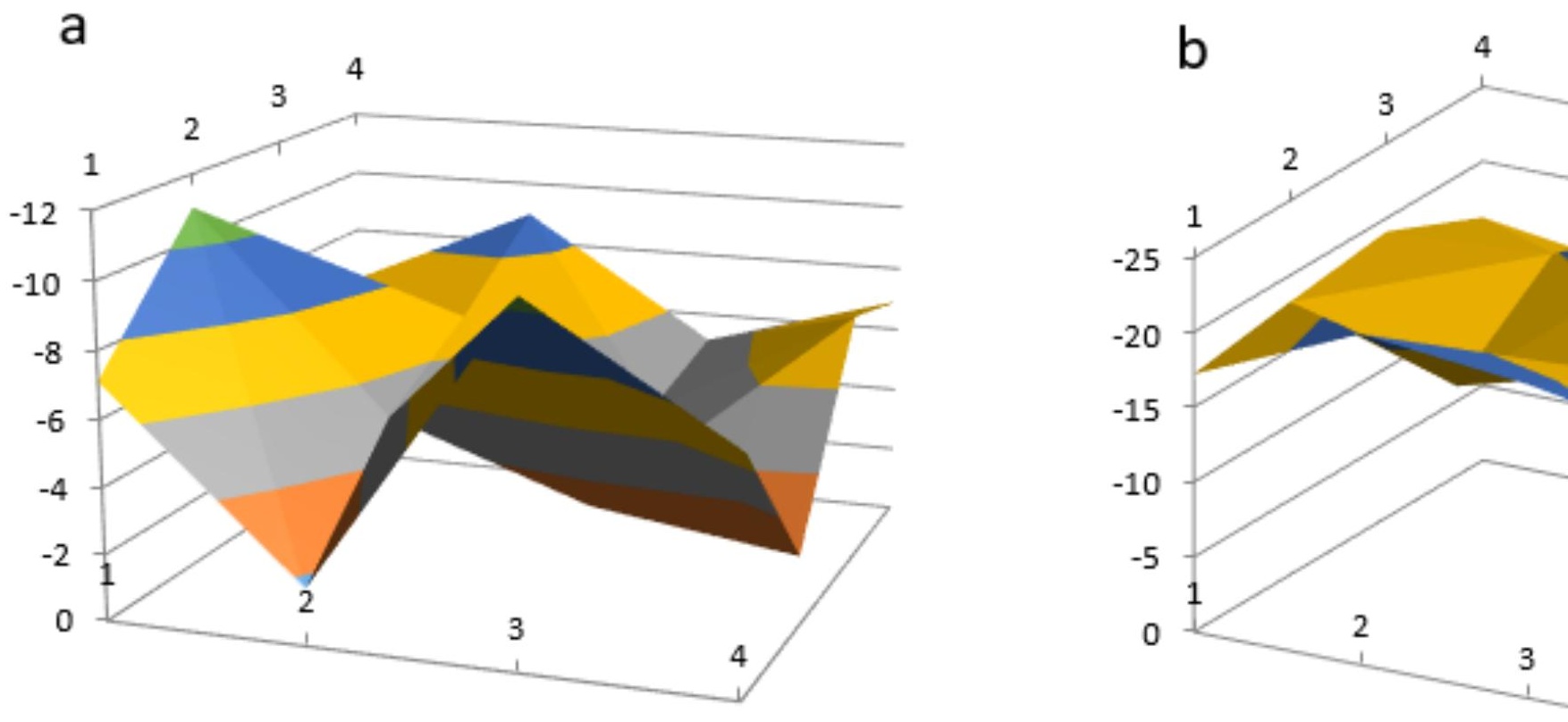

Figure.4. 9. The zeta potential distribution on the membrane surface based on $4 \times 4$ matrix AFM force map distribution is presented as $a, b$ is for the DK membrane surface. Vertical axis (Y) is zeta potential measur showing the coordinates of measuring points on the $4 \times 4$ matrix. 


\subsubsection{Influence of the Aqueous Environment on Zeta Potential Mapping}

Buffer concentrations were affecting the zeta potential from that showed in the force curves' calculation and reviewed streaming potential results (section.4.3.2 and section.4.3.3). Theoretically, the heterogeneities are also significant in the zeta potential mapping, which interrupts the comparison of zeta potential differences caused by buffers. Thus, to compare the buffer influences directly, 44 x 4 matrix mappings in different buffer conditions were used to compose an $8 \times 8$ zeta potential mapping. The $8 \times 8$ zeta potential distributions were shown as Figure.4.10 a \& b, and the scale of mapping became $18.94 \mu \mathrm{m} \times 18.94 \mu \mathrm{m}$. PH 5,7 and 9 at $0.001 \mathrm{M}$ buffers and $0.1 \mathrm{M}$ pH5 buffer were used in the measurement on Cyclopore and DK membrane surfaces to compare the $\mathrm{pH}$ and ionic strength influences.

a

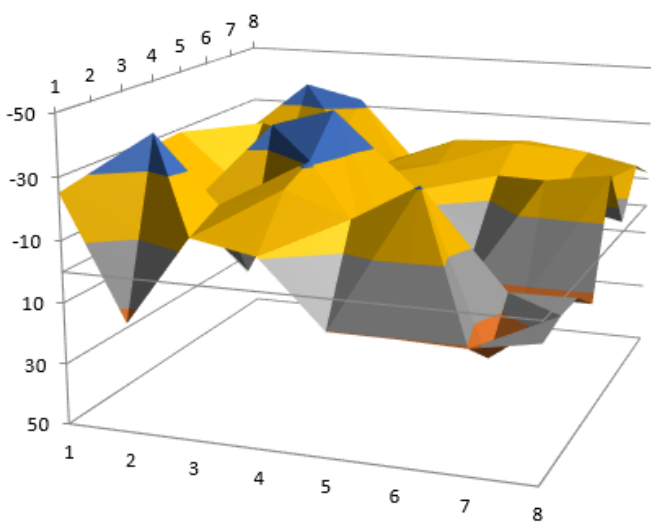

b

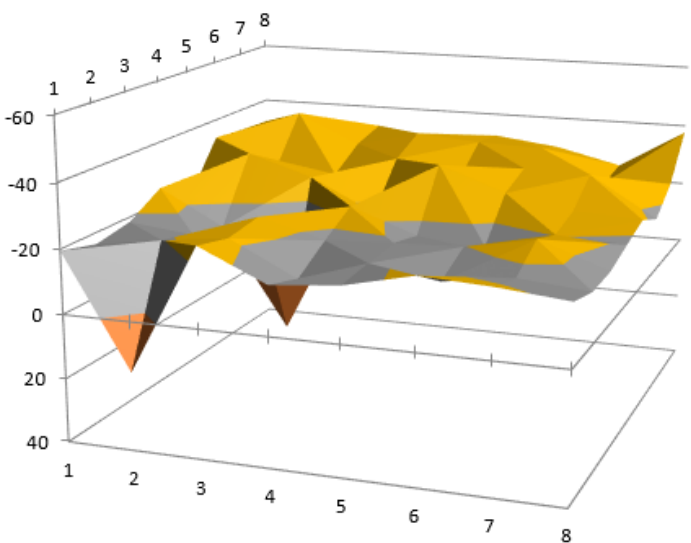

Figure.4. 10. 8 x 8 zeta potential mapping of membrane surface; Cyclopre and DK membrane displayed as a and $b$ separately. $Y$ axis is the zeta potential with unit of $\mathrm{mV}$, $\mathrm{X}$ and $\mathrm{Z}$ axis are showing the coordinates of the matrix.

The composed mapping shown in Figure.4.10a showed that zeta potential and its distribution was obviously different at different buffers, which means ionic strength and $\mathrm{pH}$ would both have significant influences on Cyclopore membrane surfaces. There was one buffer, used in the mapping, which was found to be different from the rest of the 3 buffers used in composed matrix that was shown in Figure.4.10b. From the composed mapping, the 3 matrixes tested in same concentration and 
different $\mathrm{pH}$ showed less significant differences compared to the measurement in higher ionic strength. Therefore, $\mathrm{pH}$ did not make as significant an influence as ionic strength to the surface zeta potentials on DK membrane surface. Zeta potential on Cyclopore membrane surfaces are sensitive to both $\mathrm{pH}$ and ionic strength. From Figure.4.8, it is evident that DK membranes were significantly influenced by ionic strength in high $\mathrm{pH}$ and $\mathrm{pH}$ influence would weaken when measured in high $\mathrm{pH}$ (Oatley-Radcliffe et al., 2017).

\subsubsection{Improvement of Zeta Potential Mapping}

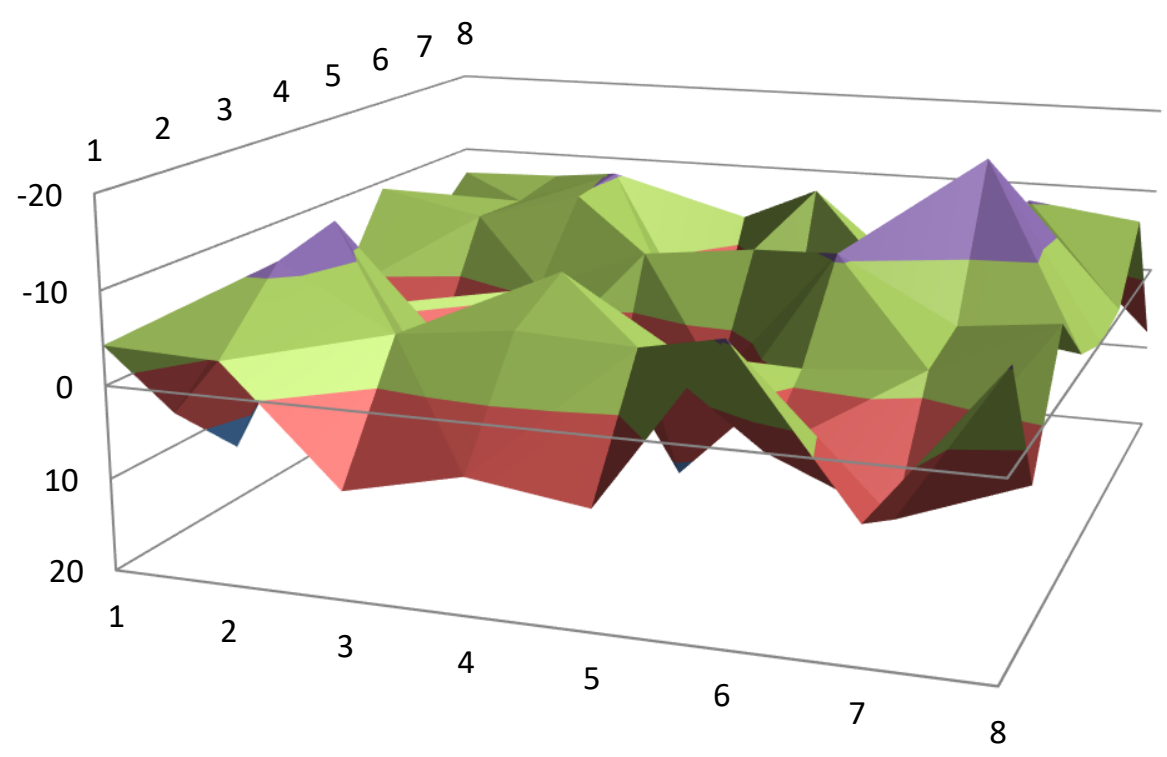

Figure.4. 11. An 8 x 8 Matrix zeta potential distribution illustration on DK membrane surface, $\mathrm{Y}$ axis is zeta potential in $\mathrm{mV}, \mathrm{X}$ and $\mathrm{Z}$ axis are the coordinates of matrix.

Zeta potential mapping on the surface was optimised with a larger matrix, which measured more points at the surface in a constant measuring condition. Thus, $8 \times 8$ matrix force mapping on DK membrane surface was measured for the calculation of zeta potential distribution on the $50 \mu \mathrm{m} \times 50 \mu \mathrm{m}$ membrane surfaces (Figure.4.11). Compared to the composed $8 \times 8$ matrix mapping (Figure.4.10 b), the zeta potential illustration with $8 \times 8$ matrix force mapping can provide a more continuity changing of zeta potential between each point. Compared to that calculated from smaller matrix 
mapping, it was significant that the zeta potential mapping with larger matrix can provide more fluctuation of zeta potential across the membrane surfaces.

\subsection{Membrane Surface Forces Analysis}

\subsubsection{Adhesion Observed on Membrane Surfaces}

Significant adhesion forces were observed from retraction force curves, as discussed in section.4.3.1. Adhesion forces were measurable from the AFM force curves directly in some studies, such as Alsteens et al.'s study on M. bovis cells (Alsteens et al., 2007). Theoretically, the adhesion is only formed after contact and will obstruct surface separation when retracing. These measured adhesion forces on membrane surfaces were then counted and compared at different buffer conditions and probes were used.

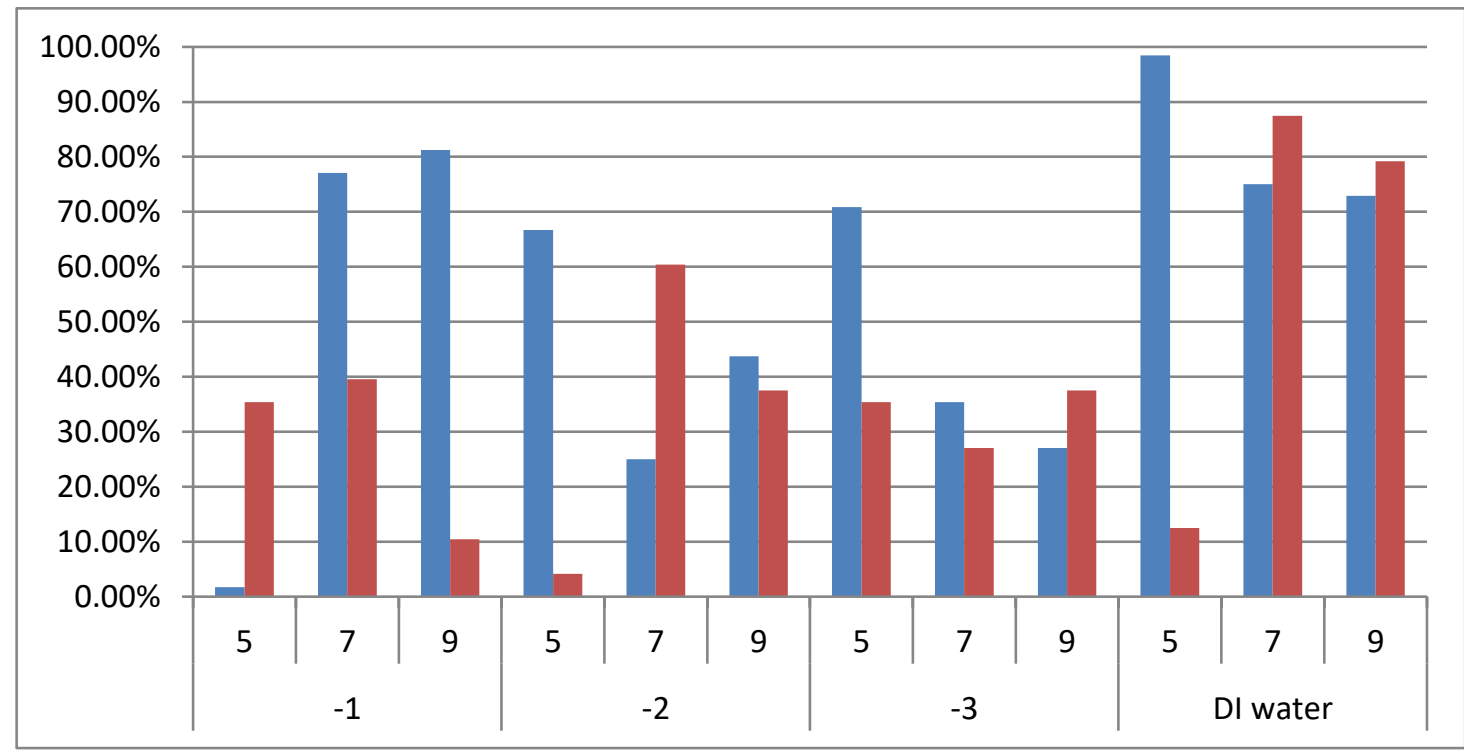

Figure.4. 12. Percentages of adhesion forces detected on membrane surfaces in different concentration and $\mathrm{pH}$ of buffer solution, used in comparison of $\mathrm{pH}$ effects at different buffer concentrations. Blue is the percentage of adhesion detected on Cyclopore membrane surface and red is for DK membrane surface.

The comparison results were presented in Figure.4.12 to show the differences that occurred due to $\mathrm{pH}$; Figure.4.13 shows the ionic strengths influences and Figure.4.14 shows the probe influences. From the comparison of percentages of adhesions in 
different $\mathrm{pH}$, ionic strengths and colloid probe sizes at $\mathrm{DK}$ and Cyclopore membrane surfaces, these influences were found to be random on both the membrane surfaces.

Therefore, buffers and probe sizes did not influence the membrane surface adhesion significantly. The adhesions on membranes were more dependent on its surface structures induced by physical and chemical forces, as that reviewed by $\mathrm{Mi}$ and Elimelech in 2008 (Mi \& Elimelech, 2008).

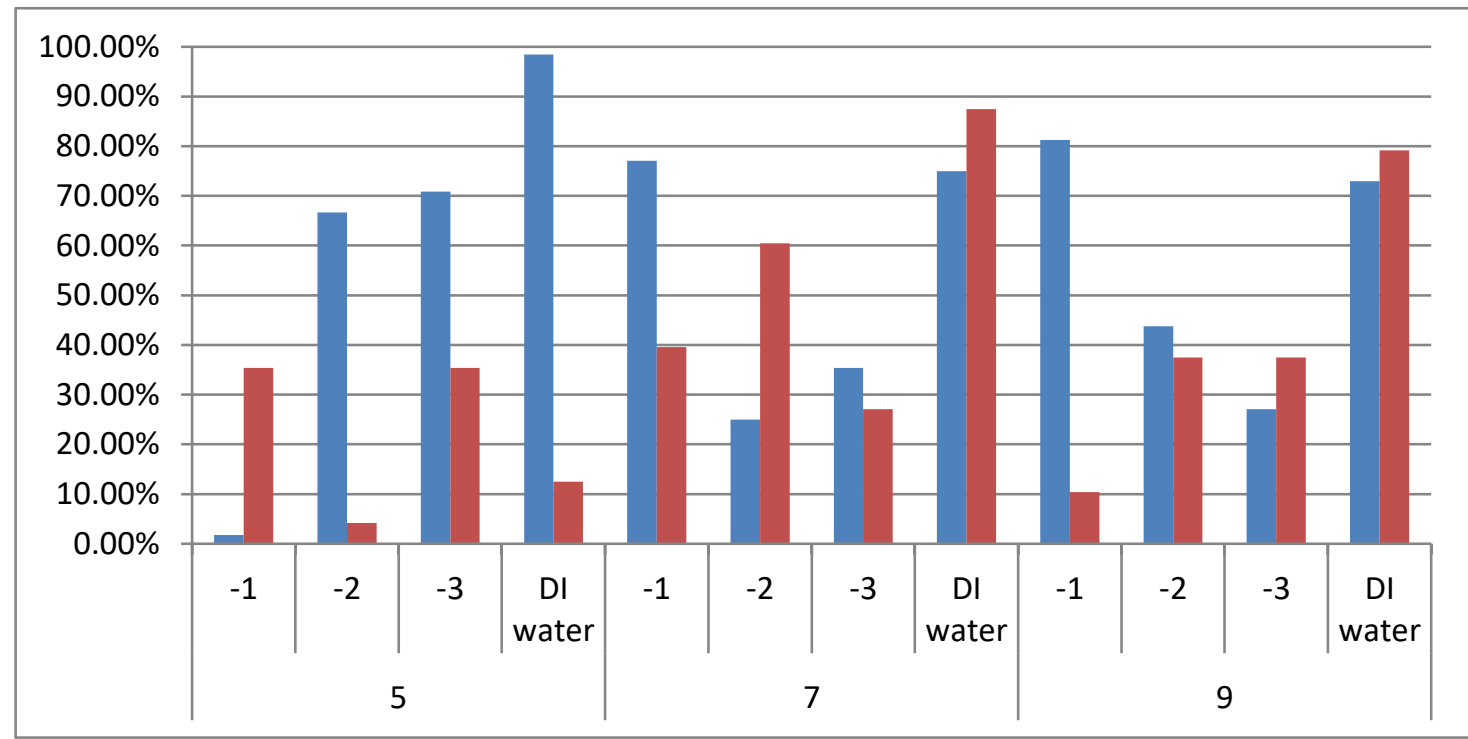

Figure.4. 13. Percentage of adhesion detected on both membrane surfaces, compare of buffer concentration effect on surface adhesion for both Cyclopore (blue) and DK (red) membrane surfaces. 

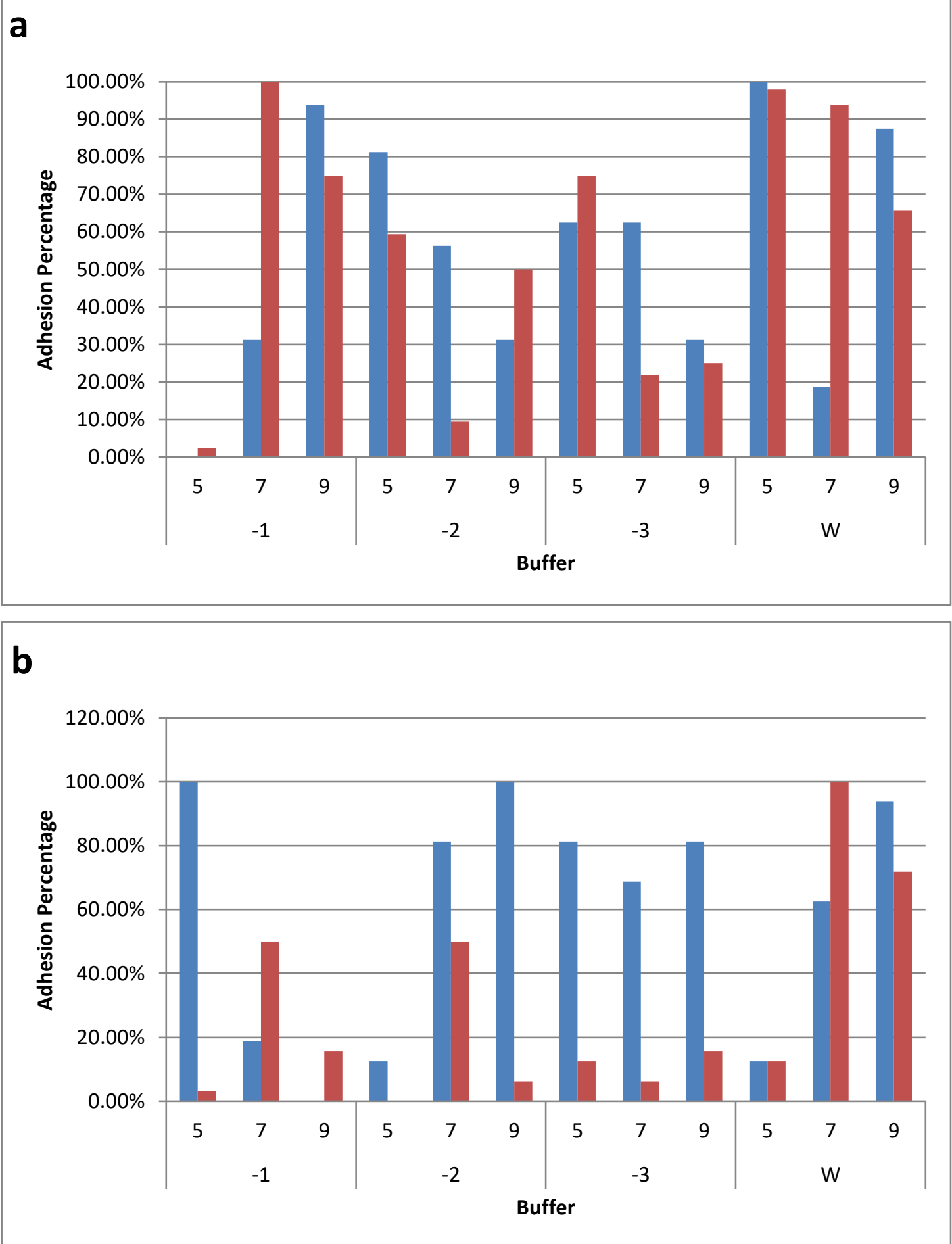

Figure.4. 14. Comparison of adhesion percentages of different probe sizes used, blue is for $5 \mu \mathrm{m}$ silica bead colloid probe and red is for $33.5 \mu \mathrm{m}$ silica bead colloid probe. Cyclopore membrane data is present in $\mathrm{a}$ and $\mathrm{b}$ is for DK membrane.

Both short-term adhesions (Figure.4.15a) and long-term adhesions (Figure.4.15b) 
were found from the retraction force curves measured on the membranes. The adhesion forces were found to be single component adhesions, or with a constant force applied. Theoretically, the constant force may be induced by a viscos layer deposited on membrane surfaces due to its feature of constant applied in constant retraction speed. The different types of adhesions were randomly distributed on the membranes measured in different buffer ionic strengths and $\mathrm{pH}$ as well. Therefore, the difference that was applied on adhesion force curves' type is also relied on the surface physical and chemical properties and its heterogeneity. 
a

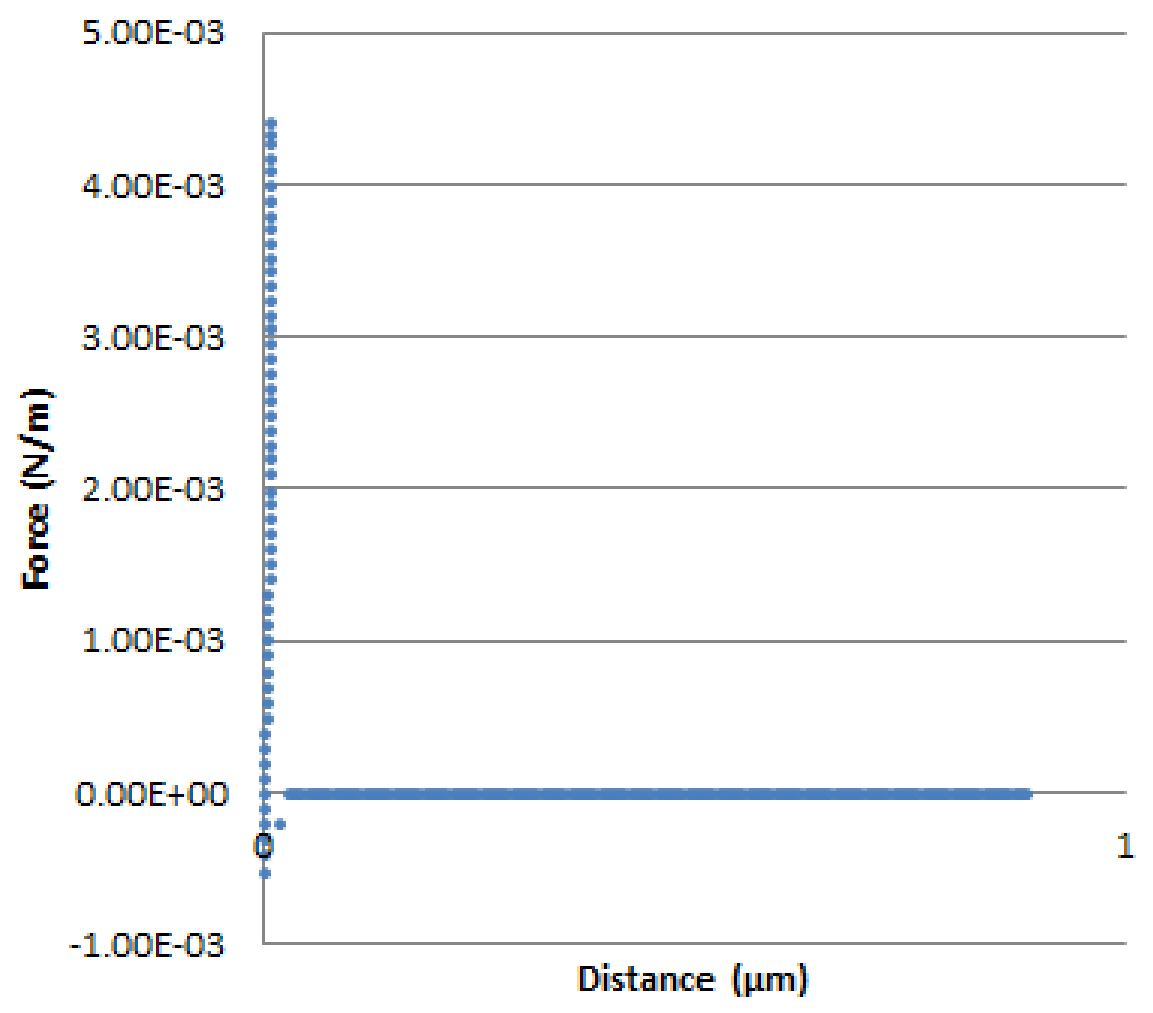

b

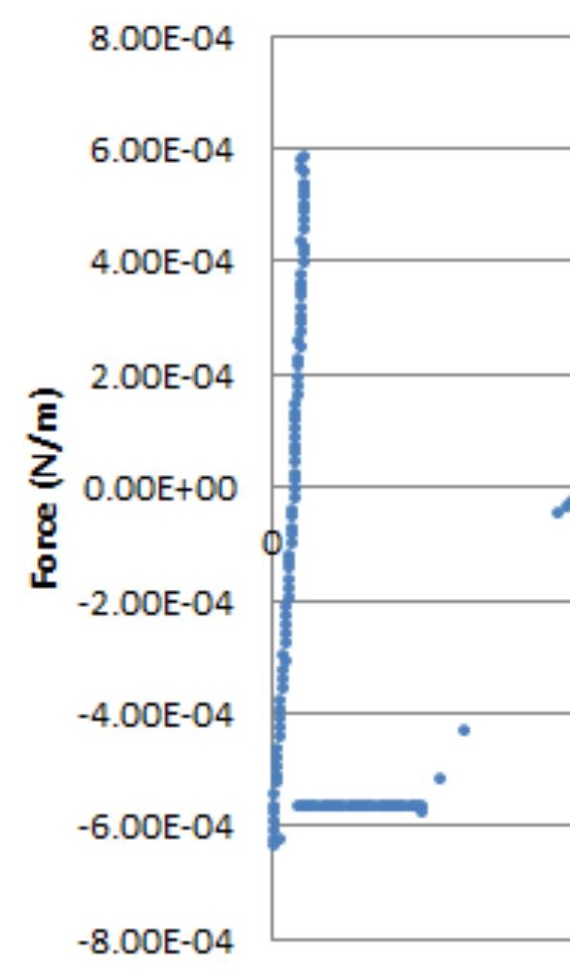

Figure.4. 15. Typical adhesions detected on both Cyclopore and DK membrane surfaces. Short term effect a (b). 


\subsubsection{DLVO Forces, Mechanic Forces and Measurement Reliability Analysis}

The forces that were applied on the colloid-membrane interactions are a convolution of many kinds of forces, which have electrostatic force concealed in them. Interactions that are found in the colloid-membrane interactions were applied on force curves as well, which means other forces, including chemical bonding forces and van der Waals forces, were also applied on the force curves measured on DK and Cyclopore membrane surfaces.

From the verification in section.3.2.6.2, the van der Waals forces were not significant compared to electrostatic force and provided a short-range influence in the colloid probe measuring system. As reviewed from previous studies, van der Waals force was only applied when the distance was as small as a few nano-meters (Harimawan et al., 2013), and was significantly affected by buffer concentrations (Butt, 1991). Long-range adhesion forces were observed on part of the retraction force curves, as shown in Figure.4.15b. In these measured adhesions, van der Waals forces were regarded as negligible, as the force existing in the distances were longer than the influencing distance of van der Waals forces. In the short-range adhesion forces, as shown in Figure.4.15a, the distance of adhesion force suspended was a few tens of $\mathrm{nm}$. It is hard to identify whether it is short-range chemical forces or van der Waals forces.

To study the force components of approaching force curves, the significant repulsive forces in approaching force curves were demonstrated in Figure.4.16. When probe was close to the membrane, before the double layers overlapped, electrostatic force was applied and it kept increasing following the DLVO theories till double layers overlapped (contact point defined in the FORTRAN program). The force that increased in this stage was shown as a smooth and non-linear curve, as shown in region 3 in Figure.4.16. Referring to the previous research of Johnson and Hidal on membrane surface repulsive force study (Johnson \& Hilal, 2015), the repulsive force change is smooth and will be affected by buffer ionic strength. The zeta potential calculation in section.4.3.2 was based on the change in forces in this region. 


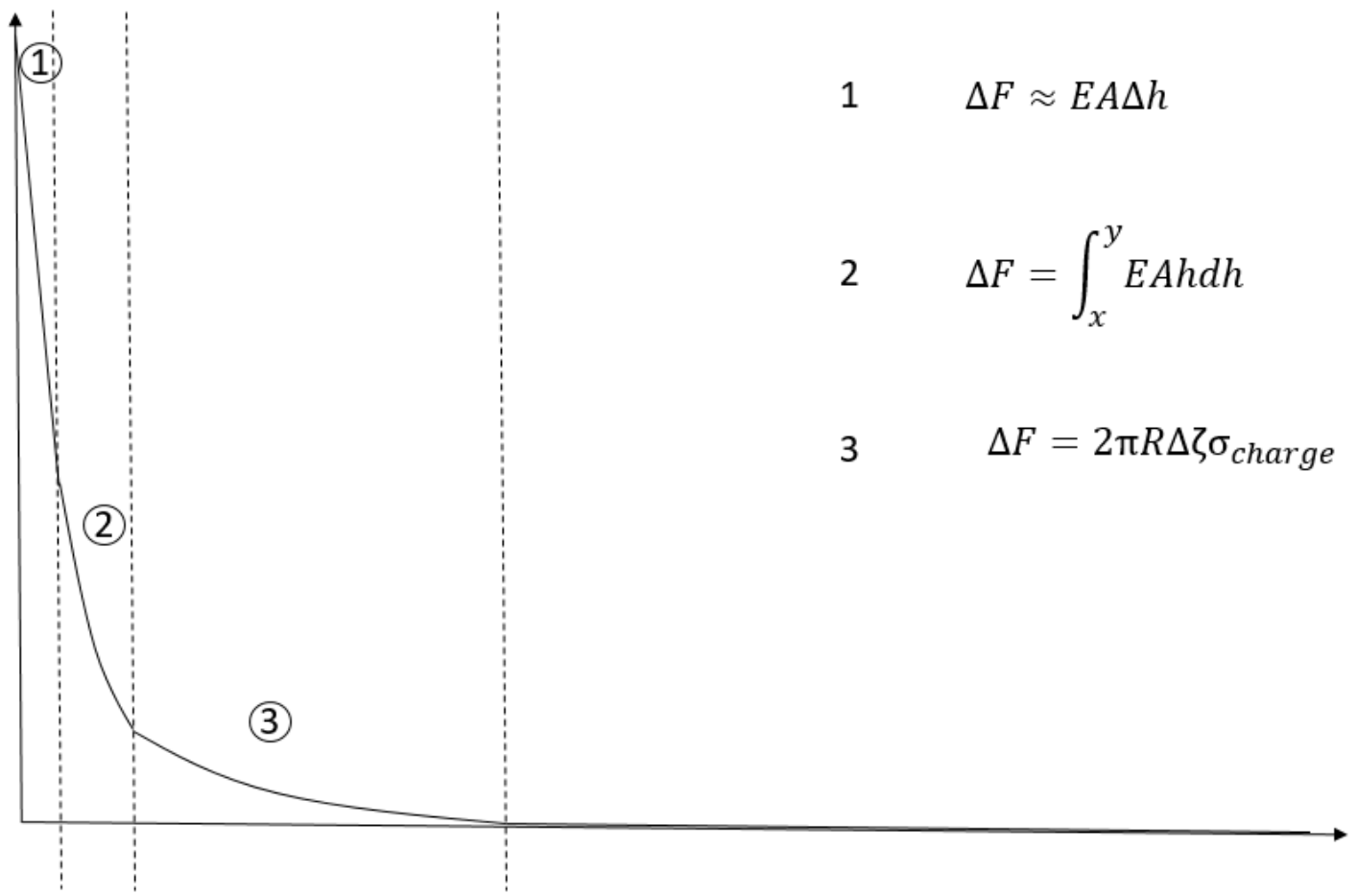

Figure.4. 16. Demonstration diagram of repulsive forces on an approaching force curve, with the mathematical illustration of these applied forces, electrostatic force (1), non-linear contact force (2) and approximate linear contact force (1). Where, E is Young's modulus (assumed as constant), $\mathrm{A}$ is contact area, $\mathrm{h}$ is penetration distance, $\mathrm{R}$ is colloid radius, $\zeta$ is zeta potential and $\sigma$ is surface charge.

After the double layer overlapped, the distance that penetrated the double layer would become the major repulsive force to obstruct the contact. Force change will be related to the penetration geometries that were applied on the double layer in mathematical relationship, as shown in Figure.4.16 with the assumed Young's modulus of double layers and referred to the geometries. The contact geometries changed rapidly when penetration was small, the force change would be a curve, as shown in region 2 in Figure.4.16. It would become more linear with penetration increase, when compression area also increases. The force would finally become approximately linear, increasing with the distance pressed into the surface when compression area is large, as shown in Figure.4.16 region 1. Membranes' Young's modulus can be estimated mathematically at this stage. However, compression of surface would change the Young's modulus and the original membrane would also be a heterogeneous surface. Thus, error will exist in the estimated Young's modulus as 
well.

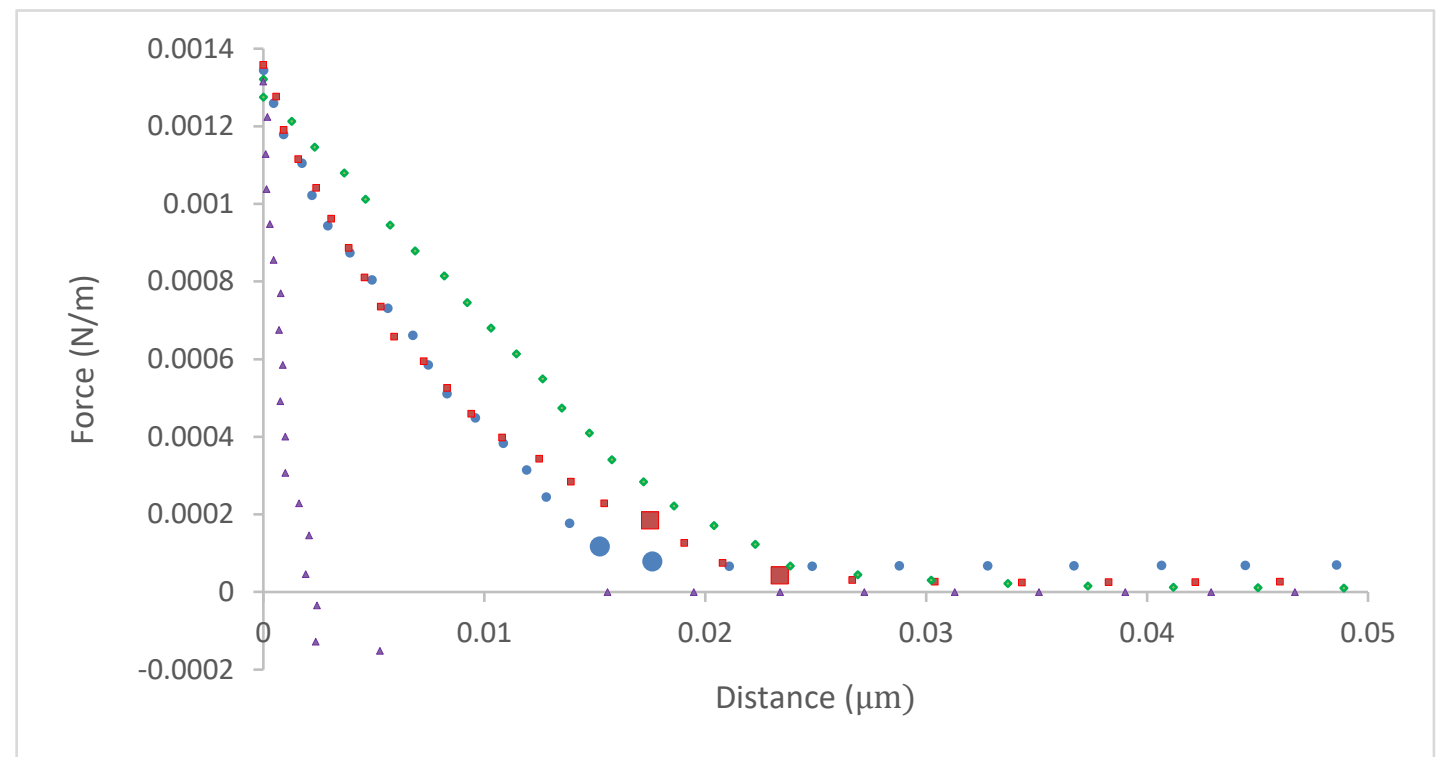

Figure.4. 17. Zoom-in into the region of distance less than $0.05 \mu \mathrm{m}$ and force less than $0.0014 \mathrm{~N} / \mathrm{m}$ for approaching force curves measured with $20 \mu \mathrm{m}$ diameter silica colloid probe on Cyclopore membrane surfaces in buffer of pH5 and 0.1 M (blue dot), $0.01 \mathrm{M}$ (red square), $0.001 \mathrm{M}$ (green diamond) $\mathrm{NaCl}$ solution and $\mathrm{DI}$ water (purple triangle). Start and end points of travelling in double-layer is marked in enlarged markers.

As described in Figure.4.16, all these forces discussed were against the compression of membrane surfaces. Application of any attractive forces would become significant in the approaching force curves. Therefore, typical force curves were obtained and zoomed-in into the region of contact on Cylopore membrane surface (region 2 in Figure.4.16), as shown in Figure.4.17. Attractive forces (snap-in) were observed in small ionic strength measurement (DI water buffer). In higher ionic strengths, the attractive forces were rarely observed, as shown in Figure.4.17. Compared to the review of van der Waals forces' estimation from Kuhn and Rahe, the shape of the approaching force curves obtained in DI water buffer can be considered as short-range chemical forces or van der Waals forces (Kuhn \& Rahe, 2014). In the measurements at DI water buffer, approaching force curves without attractive snap-in forces were also observed. It is reasonable to regard the snap-in force to be randomly measured and either caused by chemical forces or van der Waals forces.

The contact positions were also found to be extended by the thickness of diffused 
layer in the double-layer model, where zeta potential was located (OHP). The repulsive force increased faster, by about $2.5 \mathrm{~nm}$ after contact in $0.1 \mathrm{M}$ buffer, and it was about $5.9 \mathrm{~nm}$ in $0.01 \mathrm{M}$ buffer, as shown in Figure.4.17. Compared to that introduced in section.3.2.6.1, the distance between two surfaces at contact position is double of the Debye length, which is $2 \mathrm{~nm}$ in $0.1 \mathrm{M}$ and $6 \mathrm{~nm}$ in $0.01 \mathrm{M}$ buffers. The experimentally measured double layers were close to theoretical estimation. $0.001 \mathrm{M}$ buffer showed no significant change of force that increased the gradient, which means the membrane surface contacted was relatively softer and no significant force changes were caused in travelling in the double layers and contact on surface. It is also significant that from the force changes after contact, membranes' Young's modulus was different as well. Theoretically, compared to the Debye length calculated in 0.001 $\mathrm{M} \mathrm{NaCl}$ solution and the experimental measurement in $0.1 \mathrm{M}$ and $0.01 \mathrm{M}$ buffer, the distance was assumed to be between $20 \mathrm{~nm}$ and $25 \mathrm{~nm}$. Measuring in DI water showed a contact position closer to zero-distance. Ideally, edge of the diffuse layer in DI water is large, as it was measured as $0.0001 \mathrm{M}$. However, it was interfered by the attractive forces.

Cyclopore membranes are relatively rough surfaces compared to DK membranes, it normally has a roughness of a few hundred-nm, while it is only tens-nm on DK membranes. Zoom-in into contact region was done on DK membrane approaching force curves as well, as shown in Figure.4.18. Comparison of Figure.4.17 and Figure.4.18 showed that the DK membrane surface measured was softer than the Cyclopore membrane surface, because same load force strength required a longer travelling distance after contact on DK membrane surfaces. Heterogeneity that was applied on Young's modulus was significant as well from the observation on Figure.4.18. A more flattened surface was provided by the DK membrane, which showed that snap-in can be observed in force curves measured at $0.1 \mathrm{M} \mathrm{NaCl}$ buffer and snap-in disappeared in DI water buffer testing, which made it hard to identify if it was van der Waals forces or short-term chemical forces. 


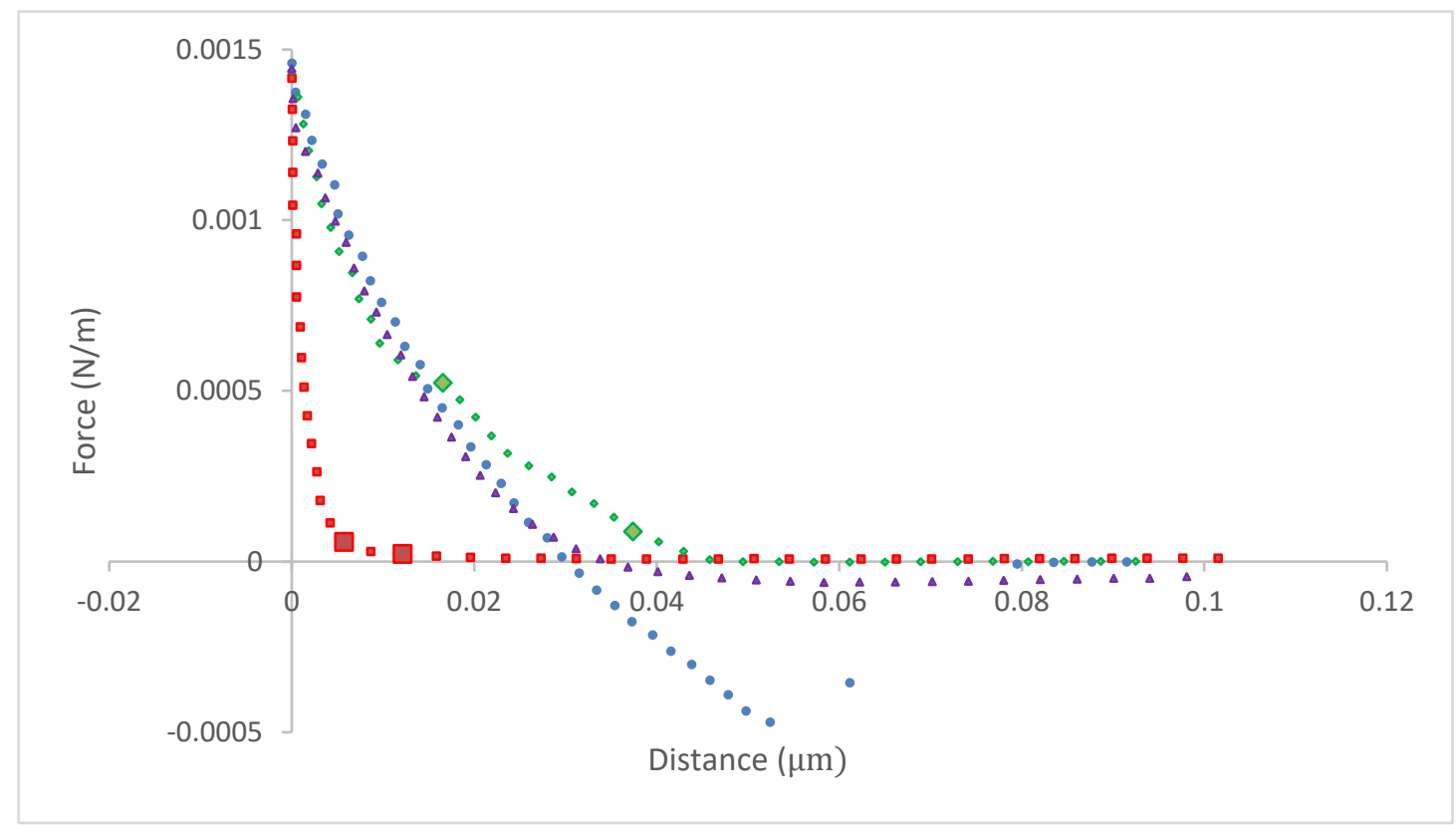

Figure.4. 18. Zoom-in into the region of distance less than $0.1 \mu \mathrm{m}$ and force less than $0.0015 \mathrm{~N} / \mathrm{m}$ for approaching force curves measured with $20 \mu \mathrm{m}$ diameter silica colloid probe on DK membrane surfaces in buffer of pH5 and $0.1 \mathrm{M}$ (blue dot), 0.01 $\mathrm{M}$ (red square), $0.001 \mathrm{M}$ (green diamand) $\mathrm{NaCl}$ solution and $\mathrm{DI}$ water (purple triangle). Start and end points of travelling in double-layer marked with enlarged markers.

The Debye length can't be determined from the observation of the approaching force curve detected in $0.1 \mathrm{M}$ buffer, as attractive forces is significant to the contact. Force measured on membrane surface at $0.01 \mathrm{M} \mathrm{NaCl}$ buffer showed that it was measured on a relatively hard surface and the surface distance, when defined as contact $(2 \kappa)$, was found to be $6.5 \mathrm{~nm}$ and it was found to be $21 \mathrm{~nm}$ when $0.001 \mathrm{M}$ buffer was applied. Moreover, Debye length in $0.01 \mathrm{M}$ is $3 \mathrm{~nm}$ and $10 \mathrm{~nm}$ in $0.001 \mathrm{M}$. However, attractive forces were still found (in $0.1 \mathrm{M}$ buffer). In DI water buffer (measured as $0.0001 \mathrm{M}$ ), the increase of the forces clearly showed the double layers; the longer Debye length will make the force change more smooth and harder to identify the influence of double layers. The use of more flattened membrane showed a more accurate determination of the double layers. Measuring in DI water showed a force curve without interference, which verified the theoretical estimation. 


\subsection{Conclusion}

The study of colloid-membrane interactions with AFM force curves in conjunction to a calculation model achieved the zeta potential calculation and adhesion force analysis. From the analysis in this chapter, the model development and its theoretical verification and calibration (as stated in section.3.2) was examined experimentally as well as with the modelled and relatively hard contact surfaces.

Heterogeneity of zeta potential was analysed as Gaussian distribution from the average value and standard deviation was calculated. Average zeta potential calculated with force curves were measured on Cyclopore and DK membranes, which showed that it was located in between 0 and $-20 \mathrm{mV}$ with a standard deviation of about $\pm 15 \mathrm{mV}$ at most buffer conditions. Compared to that measured from the Streaming potential, the difference between force curves were calculated as an average and streaming potential results were from $\pm 5 \mathrm{mV}$ to $\pm 6 \mathrm{mV}$, which was not significantly different compared to the standard deviation. When force mapping was applied, zeta potential heterogeneity was achieved as a zeta potential mapping and it was improved with higher resolution of the mapping matrix. Buffer ionic strengths and $\mathrm{pH}$ influences were found to be significant from zeta potential mapping, as well as that achieved from MWW.

Adhesions on the membrane surfaces were also studied on DK and Cyclopore membrane surfaces. Large and small adhesion strengths were found from force curves, as well as a constant adhesion force, which may be induced by viscos layer deposited on membrane surfaces. Adhesion strengths and types were found to be not significantly affected by the buffer conditions and probe sizes. Therefore, these adhesions were relied on membrane surface chemical properties, and its heterogeneity. Heterogeneity was found on membrane's Young's modulus from the difference in the force changes after surface contact. It was also calculated but the accuracy was influenced by membranes' surface heterogeneity and its measuring conditions. Debye lengths on membrane surfaces were measured from force curves. Other interaction phenomena, such as snap-in force, were found on force curves as well. Potentially, the 
snap-in force occurred due to van der Waals forces or chemical forces. 


\section{Chapter V Analysis of Intermolecular Forces of Colloid Probe Contact with Cell Surface}

\subsection{Introduction}

The interactions between colloid particles and cell surfaces are factors that affect the drug and nutrient delivery into cells (Han et al., 2005; Liu et al., 2008; Nasti et al., 2009; Yeh et al., 2011). AFM has been used in the measurement of the interactions between cells and colloid particles (Chen et al., 2009; Fakhrullin et al., 2009; Ginzburg \& Balijepalli, 2007; McNamee et al., 2006; Roiter et al., 2008; Yap \& Zhang, 2007). There are also further previous studies reviewed in section.1.3.3 that studied cell-colloid interactions. This chapter focusses on colloid-cell interactions, with the characterisation of AFM to achieve the aims and objectives of this study (section.1.5).

The interactions of three kinds of cells were measured in this chapter, bacteria of Pseudomonas fluorescens (P.F) and two kinds of yeast, NCYC-1324 and NCYC-1681. Both yeasts are Saccharomyces cerevisiae cells; NCYC-1324 is used in larger brewing (McKenzie, Main, Pennington, \& Parratt, 1990) and NCYC-1681 is used in ale brewing (Potthoff et al., 2012) and both are widely used in research of yeast characteristics. P.F bacteria are Gram-negative bacteria that are parasitic on plants (Ramjegathesh, 2014; Sivasakthi et al., 2014). A review of the surface properties of the cells that have been studied in terms of colloid contact is presented in section.1.1.

In this chapter the zeta potentials and adhesion forces incident on the AFM cell-probe are calculated from the model that was presented in section.3.2. The influences on zeta potential distributions that occurred due to buffer $\mathrm{pH}$ and ionic strengths were analysed from the force curves as well as the influences of antibiotics (amoxicillin) and adhesion control agent (sodium tripolyphosphate. Zeta potential mapping was not applied because cells were randomly immobilised on membrane surfaces. A hydrophobicity analysis, which linked the hydrophobicity and chemical bonding, was applied according to the research gap introduced in section.1.4 and 
modelled in section.3.3 to study the relationship between hydrophobicity, chemical bonding and electrostatics.

\subsection{Methods}

Experiments that were employed in this section of study used force measurements. Atomic force microscopy (AFM), Zeta-Sizer and spectrophotometry were applied to support the analysis on zeta potential, adhesion and hydrophobicity, respectively.

Yeasts and P.F bacteria were cultured using culturing media, media preparation and cell culturing that is described in section.2.2.2. AFM cell samples were then prepared with the cultured cells, as introduced in section.2.2.5.1.2. Colloid probes were fabricated with micron-size silica beads and hydroxyapatite beads as described in section.2.2.5.2. AFM contact mode imaging was achieved following the procedure in section.2.2.5.3 as well as force curves measurement that was introduced in section.2.2.5.4. Hydrophobicity determination required solvent partitioning measurement (results shown as optical density) from spectrophotometer and following the procedure as stated in section.2.2.4.

\subsection{Cell Surface Characterization}

To provide a reliable comparison of the zeta potentials calculated from force curves, yeasts and bacteria were characterised with a Zeta-Sizer to measure the average zeta potentials that were in different buffer conditions. Optical density (OD) was used to measure solvent partitioning and hydrophobicity of cell surfaces were estimated with modelling that were developed in section.3.3. The study on Zeta-Sizer measurements and hydrophobicity determination are discussed in this section. 


\subsubsection{Zeta-Sizer Measurements of Cells}

\subsubsection{Cell Size}

NCYC-1324 and NCYC-1681 yeast sizes were measured in buffers of 0.1M, $0.01 \mathrm{M}, 0.001 \mathrm{M}$ salt solutions and DI water at $\mathrm{pH} 4,5,7$ and 9, P.F bacteria was measured in the same buffer concentrations at $\mathrm{pH} 5,7$ and 9. Yeasts and P.F bacteria were cultured in MYGP with same culturing conditions. Theoretically, the buffer $\mathrm{pH}$ and concentrations produced a negligible influence on the cell size because cell growth will be halted after being removed from the culturing media.

Cell sizes distributed in buffers were measured and plotted against $\mathrm{pH}$ as shown in Figure.5.1 and against concentrations as shown in Figure.5.2. Consistent with the theoretical estimation, there was no significant influences on cell sizes that occurred from buffer $\mathrm{pH}$ or concentration. The cell size distributions for yeasts were found in the range from $1000 \mathrm{~nm}$ to $6000 \mathrm{~nm}$. However, the National Collection of Yeast Culturing (NCYC) stated that NCYC-1324 was in the size of $3-6 \mu \mathrm{m}$ and unknown NCYC-1681 cell size. Thus, the cell size measured from Zeta-Sizer showed that there was a significant amount of suspended single cells when yeasts were suspended in buffer solutions and NCYC-1681 yeasts were of similar size as NCYC-1324. Cell sizes were smaller than the range specified by NCYC; after 24 hours of culture the presence of newly divided cells and dead cells might be the reason for the smaller size detected in the present study.

The sizes of P.F bacteria were measured in the range of $3 \mu \mathrm{m}$ to $7 \mu \mathrm{m}$. Compared to the $1-2 \mu \mathrm{m}$ size measured in previous studies (Okazaki et al., 1997); significant cell aggregation may occur for P.F bacteria when suspended in buffers. P.F bacteria size was measured after a standing time of 20 minutes (section.2.2.3). Zeta-Sizer measuring collapsed and showed an error of measuring oversize. P.F bacteria had a significant faster aggregation rate in buffers compared to yeast cells. However, it can be maintained to a tolerable level within 20 mins. 


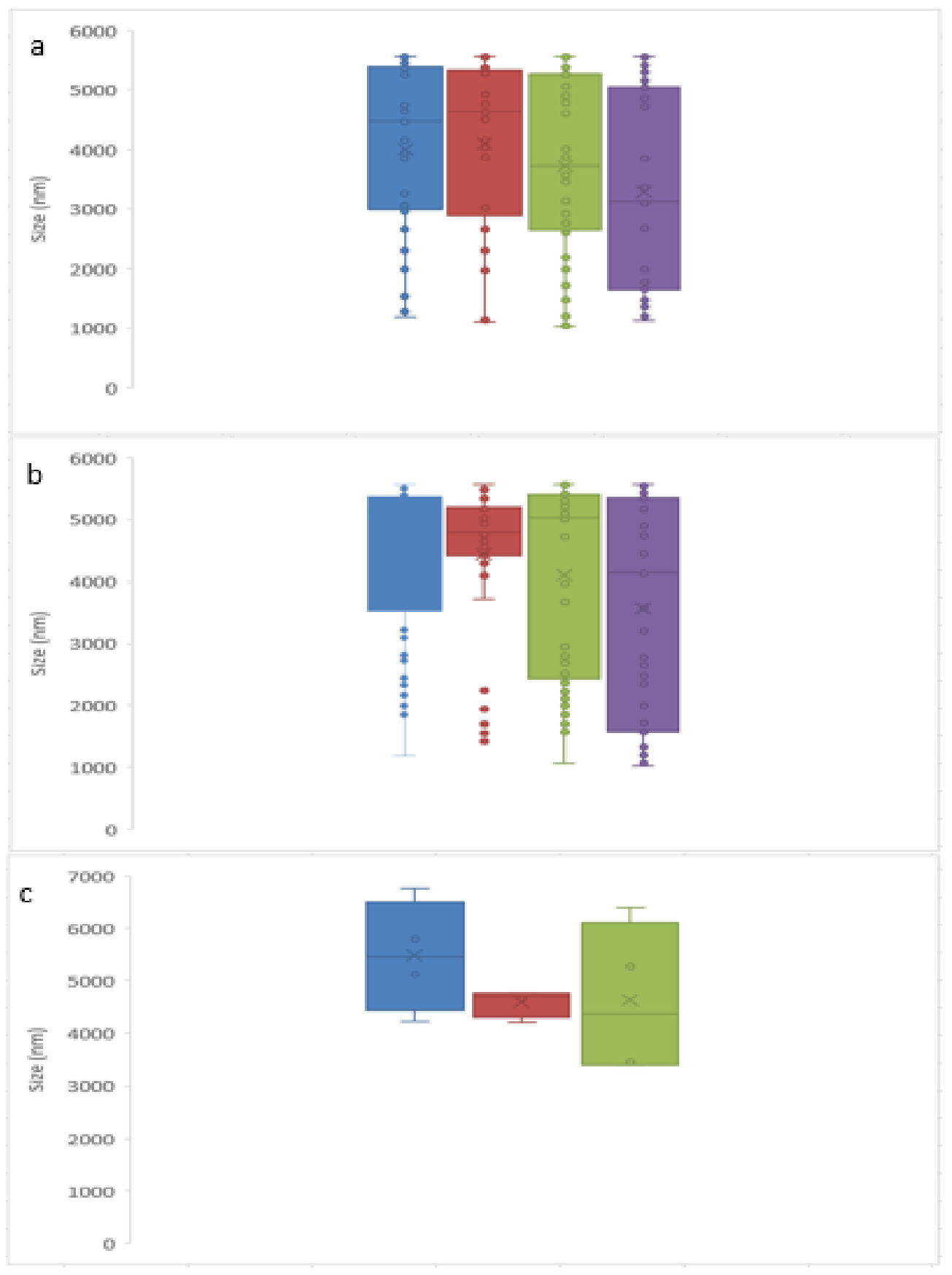

Figure.5. 1. Size distribution plot of NCYC-1324 yeast (a), NCYC-1681 yeast (b) and P.F bacteria (c) at different buffer solution $\mathrm{pH}$ condition. Size distribution is plot at $\mathrm{pH}$ 4 (blue), 5 (red), 7 (green) and 9 (purple) for yeasts (a \& b); only pH5 (blue), 7 (red) and 9 (green) were plotted for P.F bacteria (c). 


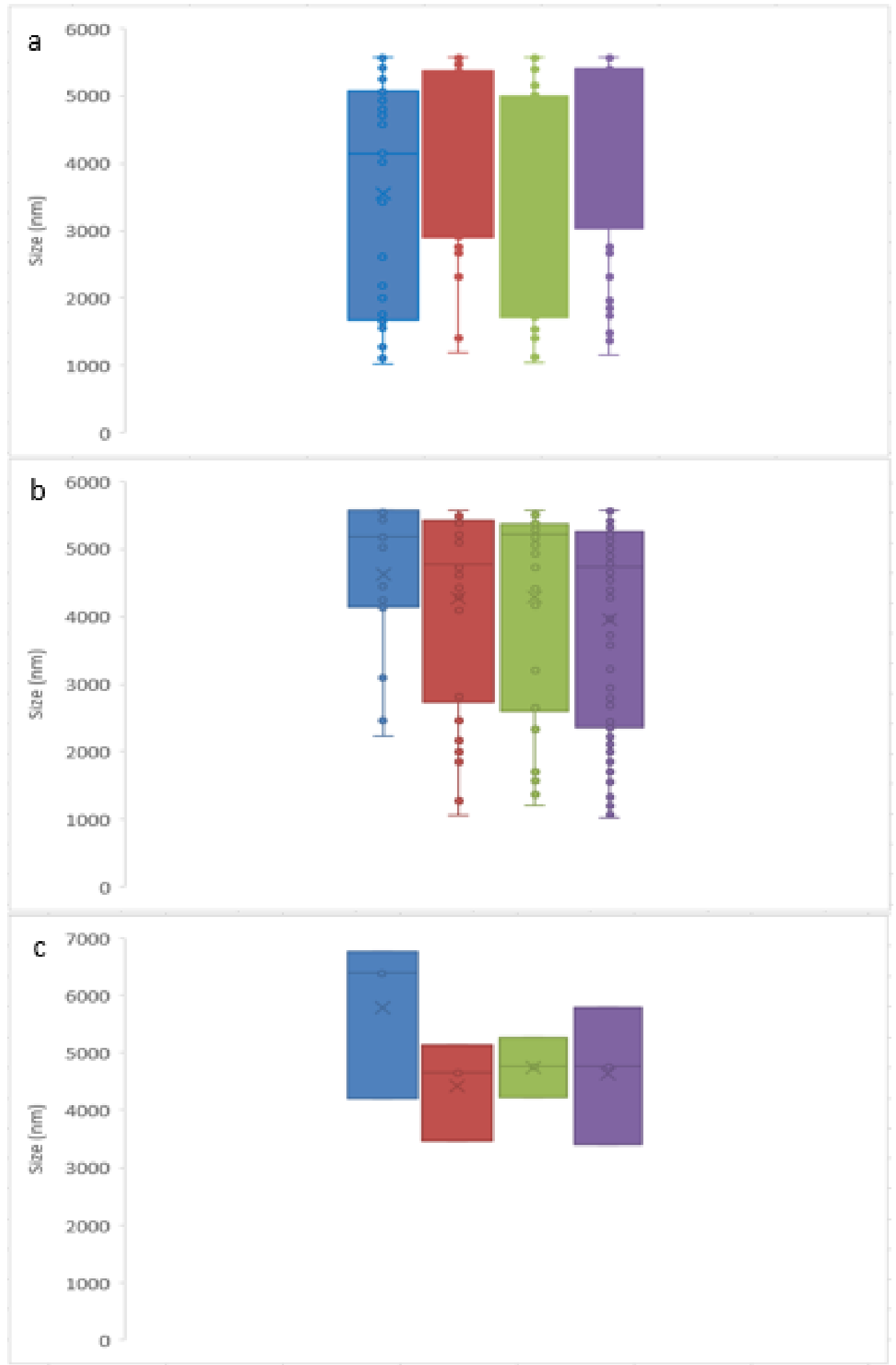

Figure.5. 2. Size distribution plot of NCYC-1324 yeast (a), NCYC-1681 yeast (b) and P.F bacteria (c) at different buffer solution concentration condition; DI water (purple), $0.001 \mathrm{M}$ (green), $0.01 \mathrm{M}$ (red) and $0.1 \mathrm{M}$ (blue) $\mathrm{NaCl}$ solutions are used in the measurements. 


\subsubsection{Average Cell Zeta Potential Measurement and Analysis}

From the Zeta-Sizer measurement in different buffer conditions, the $\mathrm{pH}$ influences on zeta potential were shown in Figure.5.3 and buffer ionic strength influences were shown in Figure.5.4. Figure.5.3 shows that a decrease in $\mathrm{pH}$ led to less negative charge at the cell surfaces. Theoretically, the cell surface chemistries were perturbed by either too high or too low $\mathrm{pH}$, with a permanent influence on the surface electrostatics properties.

In Figure.5.4, it was shown that surface charges became less negative with an increase in buffer ionic strengths. Buffer ionic strengths influenced the electrostatic double layer through changing the Debye length and buffer ions were adsorbed on the cell surfaces. From the mathematical descriptions that are applied in the DLVO theory, the Debye length does not significantly influence the charge that are located at the outer Helmholtz plane. However, the influence of the Debye length might be significant if the zeta potentials applied were high (Verwey \& Overbeek, 1948).

Theoretically, there are two phenomena that may change the surface charge on cell surfaces. Hydrolysis of surface functional groups and adsorption of charged ions as described with adsorption isotherms. Different from the influences induced from buffer concentrations, $\mathrm{pH}$ had permanent influence on the cell surfaces, which is a more complicated phenomenon than adsorption isotherms. Thus, the influence of ionic strengths is now compared using different adsorption isotherms; Langmuir isotherm and Freundlich adsorption isotherm. 

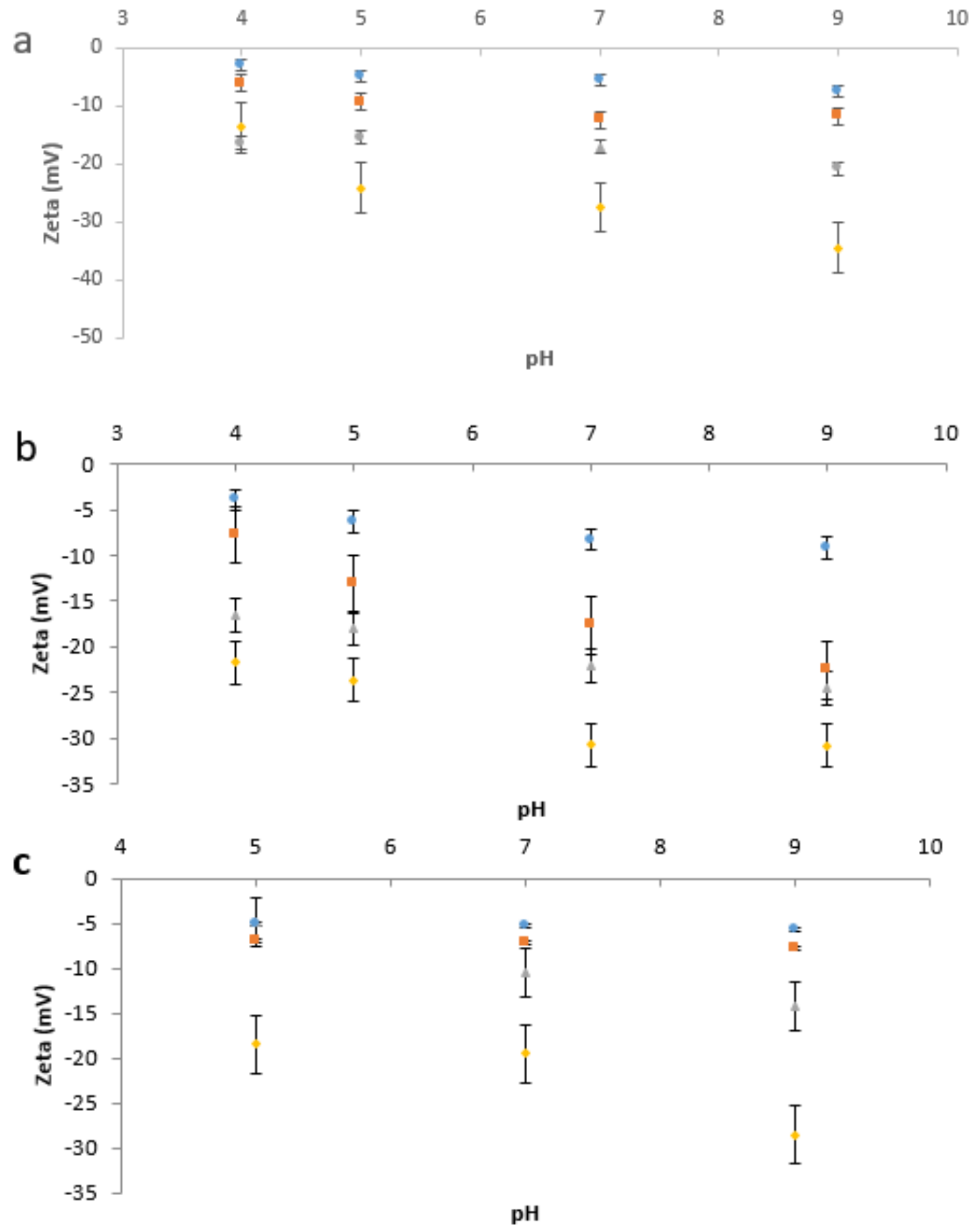

Figure.5. 3. Zeta potential measured with zeta-sizer in different buffer $\mathrm{pH}$ for NCYC-1324 (a), NCYC-1681 (b) and P.F bacteria (c). Y axis is zeta in unit of mV. X axis for is $\mathrm{pH}$. Blue dot represents $0.1 \mathrm{M}$ solution, orange square means $0.01 \mathrm{M}$ buffer, grey triangle is for $0.001 \mathrm{M}$ buffer and yellow diamond is used to identify DI water buffer, which related to about $0.0001 \mathrm{M}$ in ionic strength. 


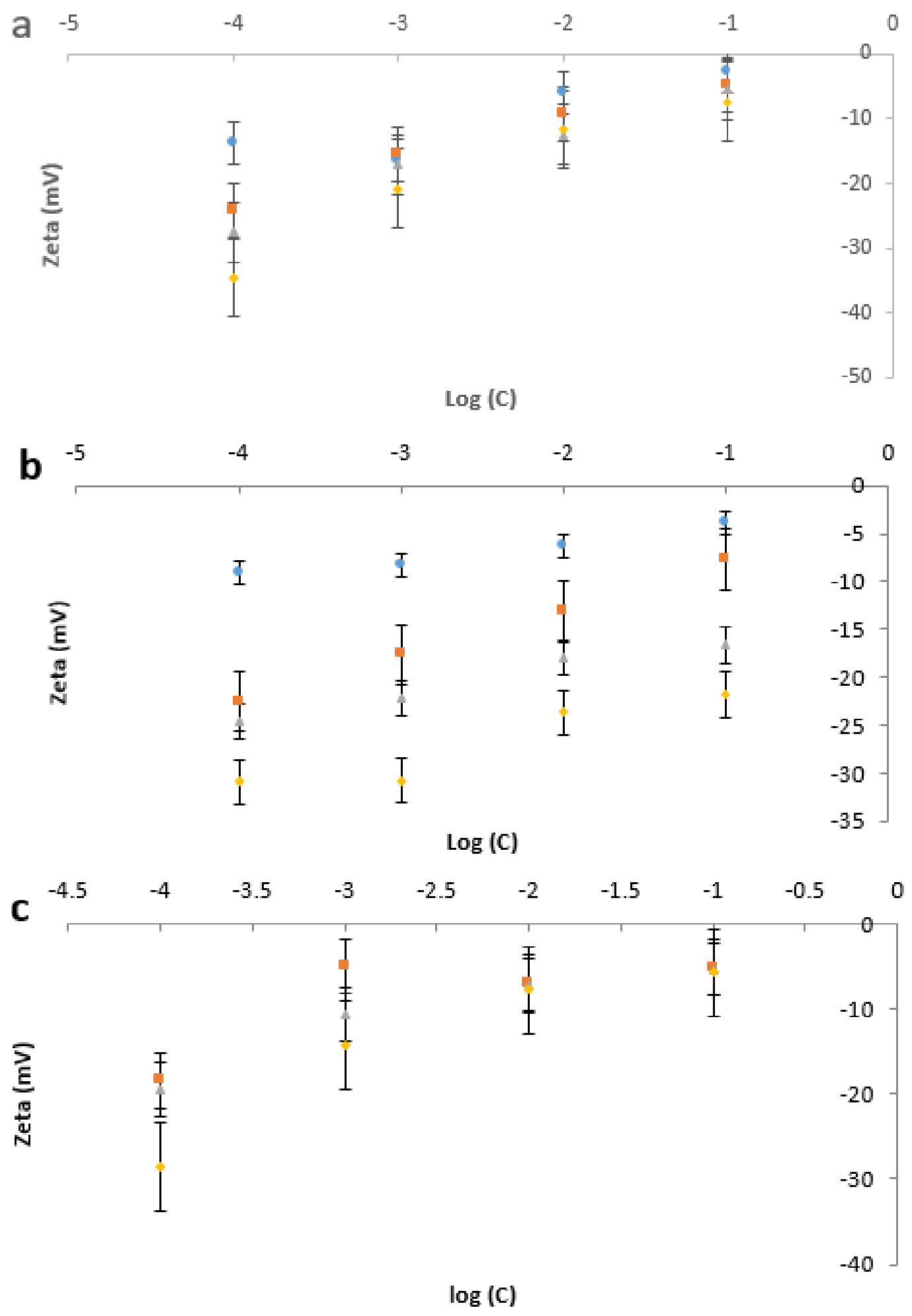

Figure.5. 4. Zeta potential measured with zeta-sizer in different buffer concentration (plot with log concentration) for NCYC-1324 (a), NCYC-1681 (b) and P.F bacteria (c). $\mathrm{Y}$ axis is zeta in unit of $\mathrm{mV}$. $\mathrm{X}$ axis for is $\mathrm{pH}$. Blue dot presents $\mathrm{pH} 4$ solution, orange square means $\mathrm{pH} 5$ buffer, grey triangle is for $\mathrm{pH} 7$ buffer and yellow diamond is used to identify pH9 buffer, while P.F bacteria is not tested in pH4 buffers (no blue dots). 
The Freundlich adsorption isotherm was applied for the analysis of the influence of ionic strength changes on yeast surface zeta potentials. There was an exponential relationship that was applied in the isotherm. DLVO forces may also have influences on the zeta potential measured in different ionic strengths when the zeta potential applied was large. DLVO theory shows that the electrostatic potential is related exponentially to the square root of the buffer concentration. To be consistent, zeta potentials were plotted against the square root of buffer concentration as shown in Figure.5.5.

a

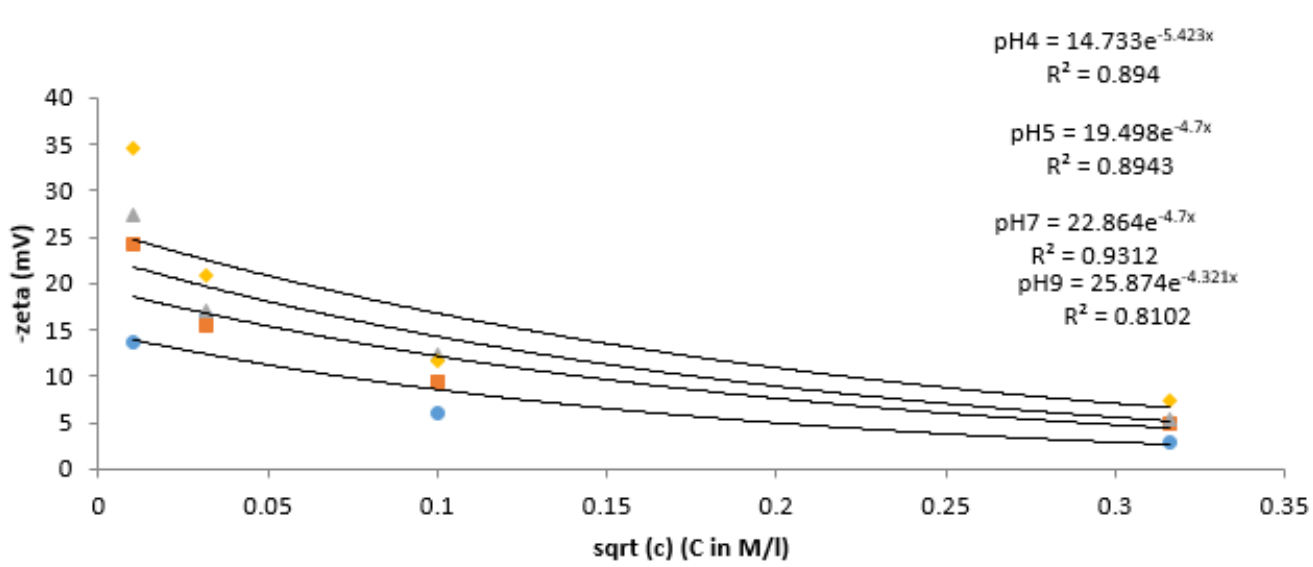

b $\mathrm{pH} 4=18.655 \mathrm{e}^{-5.239 x}$
$\mathrm{R}^{2}=0.8985$

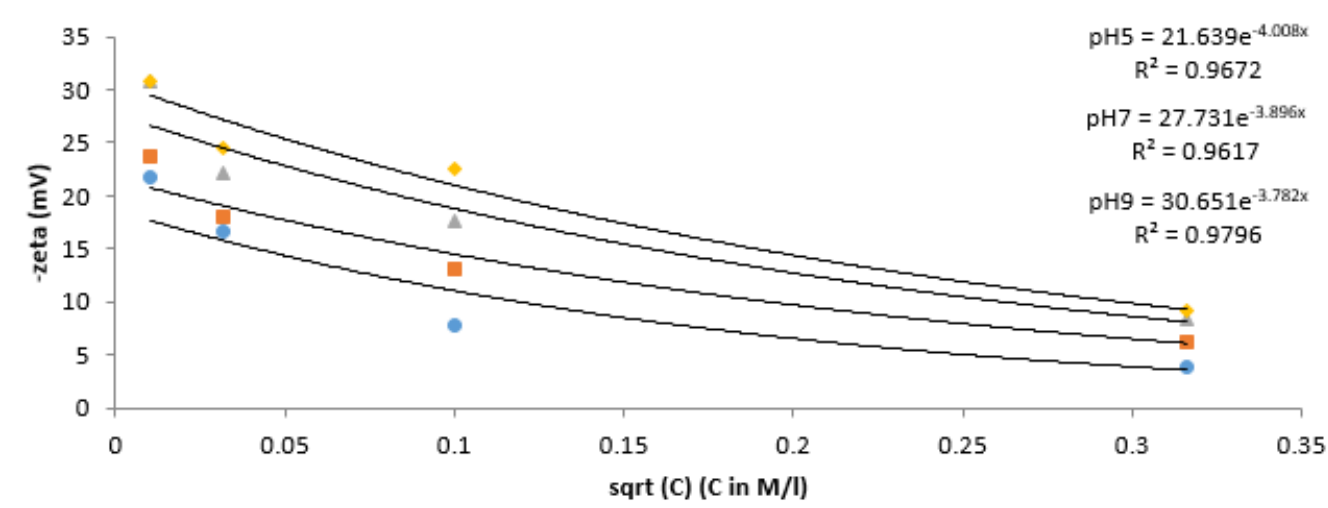

Figure.5. 5. The plot is for -zeta potential of NCYC-1324 (a) and NCYC-1681 (b) yeast cell against square root buffer concentration. Curve fitting is applied with an exponential relationship. Blue dot is for $\mathrm{pH} 4$, orange square is for $\mathrm{pH} 5$, grey triangle is for $\mathrm{pH} 7$ and yellow diamond presents $\mathrm{pH} 9$.

As shown in Figure.5.5, the regression coefficients $\left(\mathrm{R}^{2}\right)$ for the curve fittings were higher than 0.8 for both yeasts measured at different $\mathrm{pH}$. It is significant that the 
ionic strength influences zeta potentials, as described by the Freundlich adsorption isotherm. Theoretically, zeta potentials were measured on OHP, which is one Debye length from the charged surface. However, from the review of DLVO theories (Verwey \& Overbeek, 1948), the distance from OHP to charged surface would be different from the Debye length at high zeta potentials. Thus, the exponential term at high zeta potential would induce a difference in the curve fittings because it was no longer a constant, which is bay be why the maximum zeta potential measured (NCYC-1324 at $\mathrm{pH} 9)$ had the lowest regression coefficient $\left(\mathrm{R}^{2}=0.8102\right)$ and the consideration of Debye length influence was not described by the model.

The data were then compared with the Langmuir isotherm, which is derived from the reaction equilibrium and with the theoretical consideration that the OHP would not make a significant influence. The relationship between the inverse of the zeta potential and buffer concentrations was derived as Equation.5.1 in a linear relationship. The exponential influence was considered as a constant as described in the Freundlich adsorption isotherm analysis $(\exp (-1))$. The potential in the Langmuir isotherm is in a non-linear and non-exponential relationship with the buffer concentration.

$$
\frac{1}{\psi}=\frac{1+K C_{\text {buffer }}}{\psi_{\text {total }}} \exp (-1)
$$

Equation.5.1

Where, $\Psi$ is the electrostatic potential and $\mathrm{K}$ is equilibrium constant. Zeta potentials were plotted, and the curve fitted with Equation.5.1 and was shown in Figure.5.6 for yeasts. The curve fitting showed that it was accurate to summarise the zeta potential as a linear relationship with $\mathrm{R}^{2}$, more than 0.9 for most curves. The same as for the Freundlich adsorption isotherm, the largest zeta potentials (measured on NCYC-1324 at pH9) were curve fitted with the lowest regression coefficient $\left(\mathrm{R}_{2}=\right.$ $0.8255)$.

Comparing Figure.5.6 to Figure.5.5, both the Langmuir isotherm and Freundlich adsorption isotherm provided similar regression coefficients for the estimation of zeta potentials change in different ionic strengths. Therefore, the influence of the position of OHP used in the calculate ion (Langmuir used the actual surface, Freundlich used 
the Debye length distance) did not significantly change the zeta potential.

a $\begin{aligned} & \mathrm{pH} 4= 2.6392 \mathrm{x}+0.0882 \\ & \mathrm{R}^{2}=0.9295\end{aligned}$
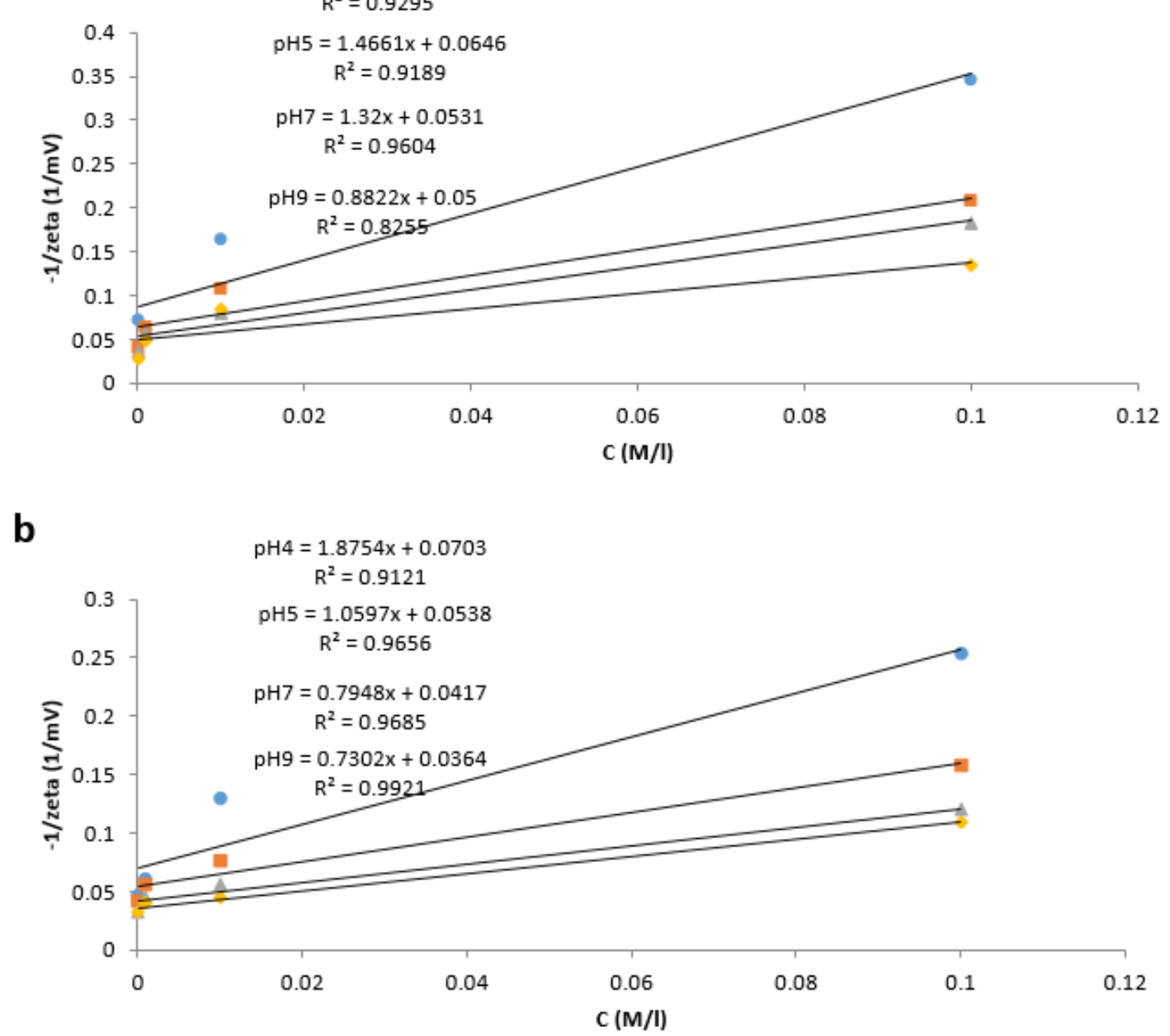

Figure.5. 6. The plot is to illustrate the linear relationship between $-1 / \psi$ and buffer concentration for NCYC-1324 (a) and NCYC-1681 (b). Blue dot is for $\mathrm{pH} 4$, orange square is for $\mathrm{pH} 5$, grey triangle is for $\mathrm{pH} 7$ and yellow diamond presents $\mathrm{pH}$.

Both isotherms were applied in the comparison of zeta potential measured for P.F bacteria as shown in Figure.5.7. Both comparisons showed that adsorption isotherms did not provide curve fittings as good as that achieved from NCYC-1324 and NCYC-1681 yeasts measurements. There were more phenomena that contributed to the ionic strength influence that was applied on zeta potential changes at P.F bacteria surfaces. Potentially, it was more related to surface-chemistry change. Thus, hydrophobicity was studied in the following section to achieve more understanding of surface-chemistry and the influences of ionic strength on cell zeta potentials. 
a

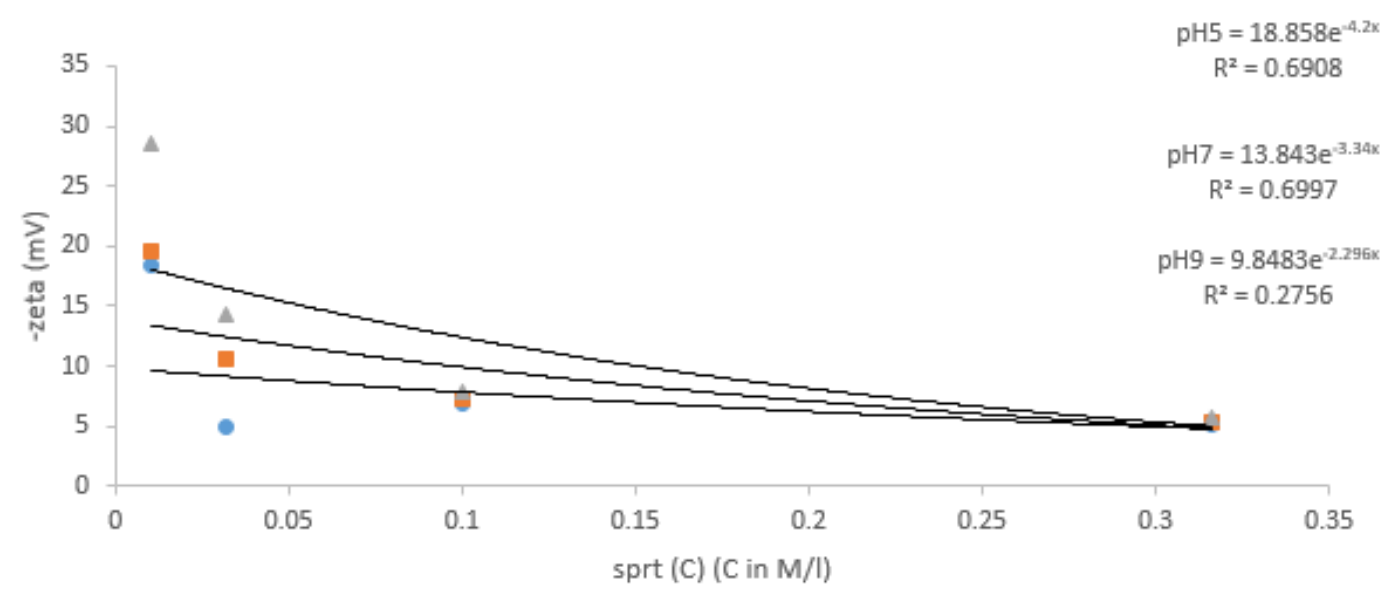

b

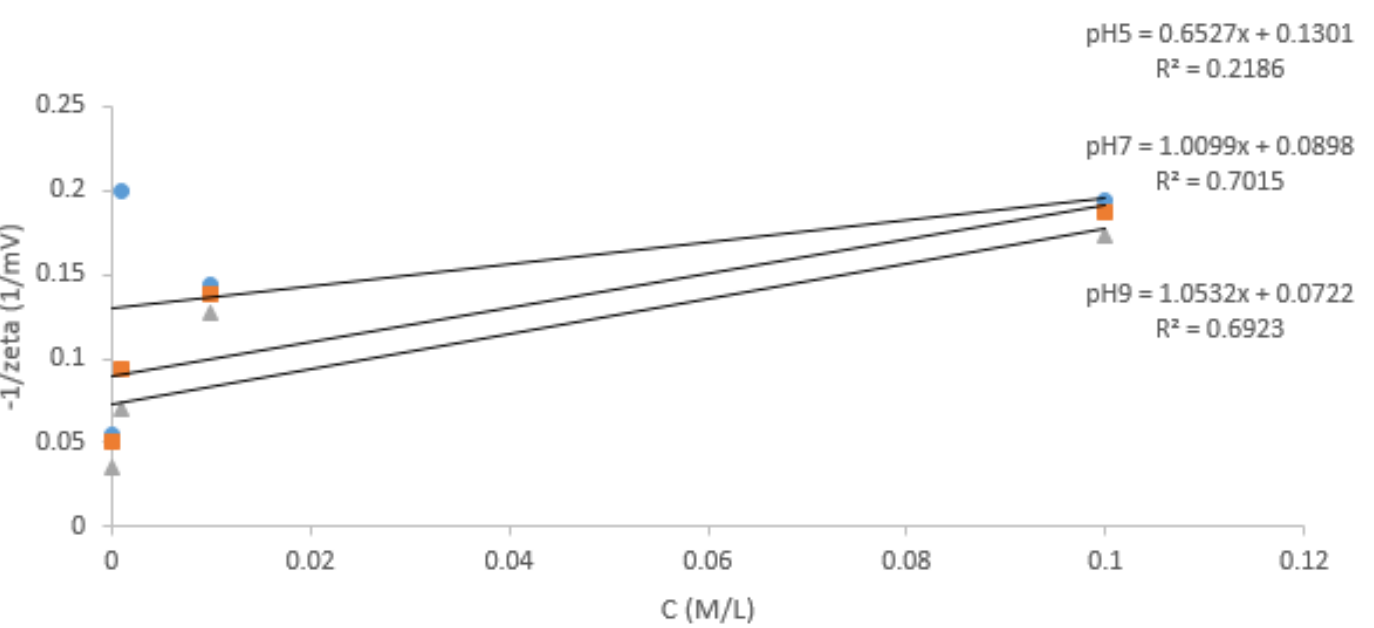

Figure.5. 7. P.F zeta potential plot of (a) -zeta against sqrt (C) and (b) -1/zeta against $\mathrm{C}$, in pH5 (blue dot), 7 (orange square) and 9 (grey triangle).

\subsubsection{Measurement and Analysis of Cell Hydrophobicity}

\subsubsection{The Influence of the Aqueous Environment on Cells in Optical Density}

Hydrophobicity of the 3 kinds of cells was measured with an optical density (OD) method. The OD was measured as a percentage of cells remaining in the water after cells had been washed out by hexadecane in solvent partitioning, following the experimental protocols presented in section.2.2.3. Hexadecane residue and cell aggregation caused errors, which were all found to be time dependent; these influences were measured in Appendix.1.1 for hexadecane influence and 
Appendix.1.2 for aggregation influence. The measured influences were used to optimise the measurement of hydrophobicity from solvent partitioning.
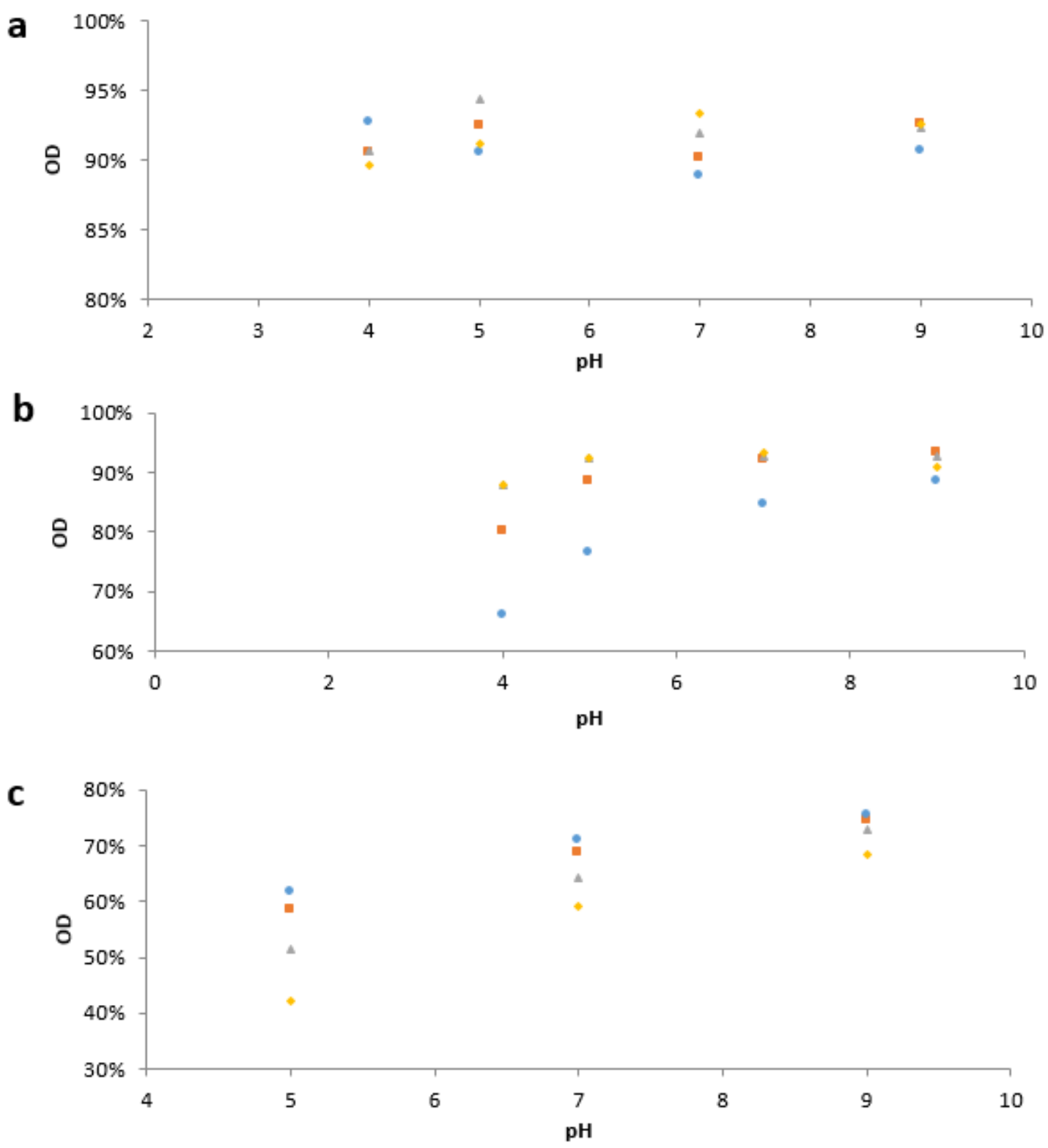

Figure.5. 8. Plot of optical density against pH for NCYC-1324 (a), NCYC-1681 (b) and P.F bacteria (c). Solvent partitioning was in different buffer concentration, blue dot for $0.1 \mathrm{M}$, orange square for $0.01 \mathrm{M}$, grey triangle for $0.001 \mathrm{M}$ and yellow diamond for DI water

The OD of cells remaining within the aqueous suspension was measured and plotted against $\mathrm{pH}$ and ionic strengths as shown in Figure.5.8 and Figure.5.9. The OD of remaining NCYC-1324 cells was randomly distributed at different ionic strengths and $\mathrm{pH}$ (Figure.5.8a and Figure.5.9a). The range of OD for remaining cells was in a 
narrow range from $89 \%$ to $95 \%$. Approximately $90 \%$ of NCYC-1324 yeast cells remained in the water-based solution, as stated in section.3.3.1. The effects of $\mathrm{NaCl}$ concentrations and buffer $\mathrm{pH}$ were negligible on the number of $\mathrm{NCYC}-1324$ cells that remained in the water. Thus, there were about 10\% of NCYC-1324 yeast, which were washed away from the aqueous buffer and those cells were regarded as dead cells that had lost their surface chemical activities, the live NCYC-1324 yeasts were rich in hydrophilic chemical groups on the cell surface. The measurement showed that surfaces of NCYC-1324 cells were saturated with hydrophilic groups and the about $90 \%$ of the cells were influencing the measurement (live cell rate) while $10 \%$ were effectively dead.

a

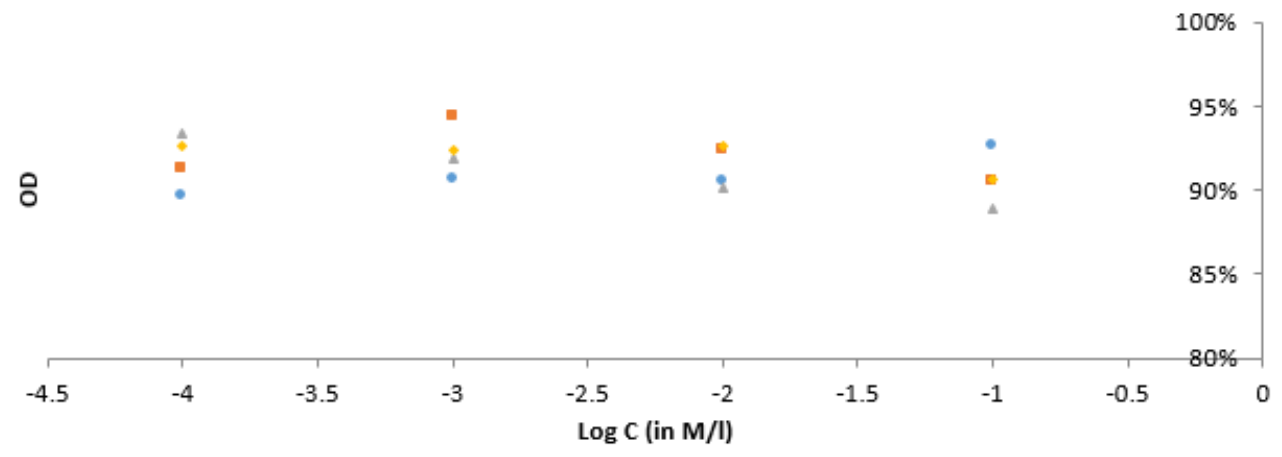

b

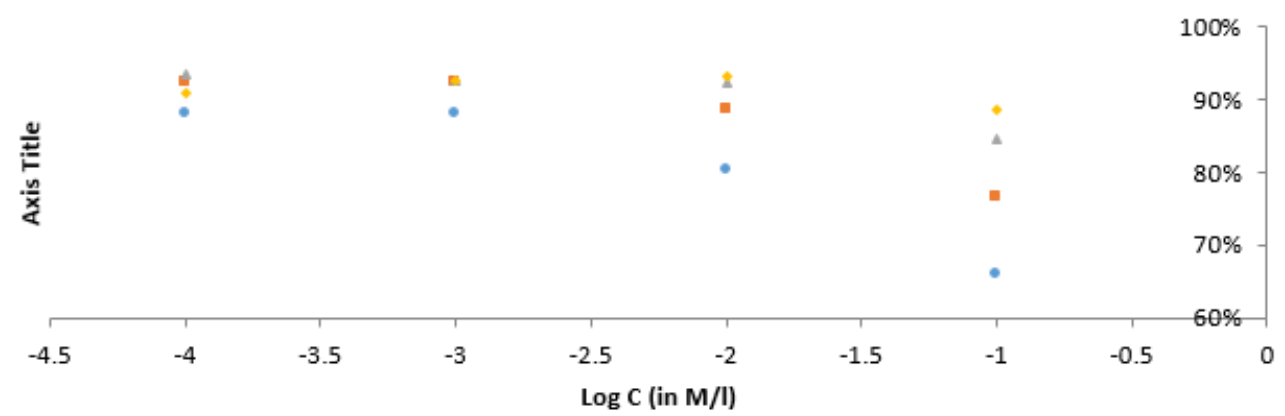

C

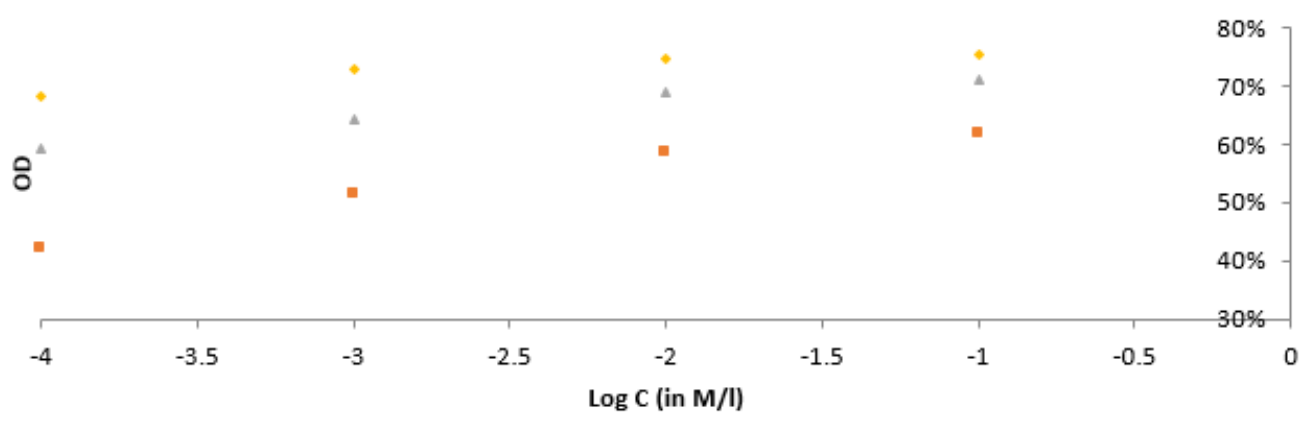

Figure.5. 9. Plot of optical density against log of buffer concentration for NCYC-1324 (a), NCYC-1681 (b) and P.F bacteria (c). Partitioning experiments were in different buffer $\mathrm{pH}$, blue dot for $\mathrm{pH} 4$ (P.F bacteria not tested in $\mathrm{pH} 4$ ), orange square for $\mathrm{pH} 5$, grey triangle for $\mathrm{pH} 7$ and yellow diamond for $\mathrm{pH}$. 
Unlike NCYC-1324, ionic strengths and $\mathrm{pH}$ were found to be more influential on NCYC-1681 hydrophobicity. As shown in Figure.5.8b and Figure.5.9b, hydrophobicity increased with an increase in the $\mathrm{pH}$ value and decreased with higher concentration of $\mathrm{NaCl}$. Cells remaining rate dropped to $65 \%$ at $0.1 \mathrm{M}$ and $\mathrm{pH} 4$ buffer solution. Cell remaining rate increased as a curve with decrease in buffer concentrations or increase in $\mathrm{pH}$. The cell remaining rate also became stable around the value of $90 \%$. Therefore, the saturated cell remaining rate (live cell rate) for NCYC-1681 was also found to be around 90\%.

For P.F bacteria, as shown in Figure.5.8c and Figure.5.9c, the maximum cell remaining rate at different $\mathrm{pH}$ values were different from each other, from $64 \%$ at $\mathrm{pH} 5$ to $82 \%$ at $\mathrm{pH}$. From the plots for P.F bacteria in Figure.5.8c and $9 \mathrm{c}$, the cells remaining rate increasing trend for P.F was increasing slower with higher $\mathrm{pH}$ and buffer concentrations and approached the value of $90 \%$. Thus, the same live cell rate (90\%) was achieved for all 3 cell types.

Comparison of Figure.5.8 and Figure.5.9 showed that the $\mathrm{pH}$ influenced more on the hydrophobicity compared to that of buffer concentrations. Theoretically, the buffer concentrations would provide less biological influence on microbial cell surfaces and those influences induced by buffer concentration were regarded as a reaction equilibrium, as stated in section.3.3.2. Normally, $\mathrm{pH}$ is regarded as more harmful to cell health, which would damage the cell surface properties. It means that the change caused by $\mathrm{pH}$ was more complicated and unlike the influence from ionic strength, was regarded as a non-reversible (permanent) influence on cell surfaces. As observed from the cell remaining rate plot, hydrophobicity and its related chemical properties were not described directly. Therefore, more complicated analysis was applied on the liquid partitioning measured results for both yeasts and bacteria to deconvolve those cell surface properties from experimental results, which are now described in the following section. 


\subsubsection{Numerical Analysis of Relationship between Aqueous Phase Optical Density and Cell Surface Hydrophobicity}

\subsection{Application of Developed Model in Hydrophobicity Analysis}

Hydrophobicity is related to a cell's surface chemistries. The cell membrane and polymeric cell wall structures could be regarded as hydrophobic from their components as well as the outer wall structure of Gram-negative bacteria, which both do not induce a hydrophilic drag force as described in section.3.3.1. From the review in section.1.1, there are many kinds of molecules found on the plasma membrane surfaces and cell wall structures. The analysis of the influence of ionic strengths on hydrophobicity in section.5.3.2.1 and its theoretical description in section.3.3.2, are easier to model due its reversible nature, however the $\mathrm{pH}$ influence was more complicated and permanent for the surface chemical structures. The model developed in section.3.3.3 was used in the calculation of hydrophobicity of cell surfaces.

\begin{tabular}{|c|c|c|c|c|c|c|c|c|c|c|c|c|}
\hline $\log C$ & \multicolumn{3}{|c|}{-1} & \multicolumn{3}{|c|}{-2} & \multicolumn{3}{|c|}{-3} & \multicolumn{3}{|c|}{ DI } \\
\hline ceis & 1324 & 1681 & P.F & 1324 & 1681 & P.F & 1324 & 1681 & P.F & 1324 & 1681 & P.F \\
\hline 4 & \multirow{4}{*}{ HS } & 0.57 & NA & \multirow{4}{*}{ HS } & 1.11 & NA & \multirow{4}{*}{ HS } & 1.67 & NA & \multirow{4}{*}{ HS } & 1.66 & NA \\
\hline 5 & & 0.95 & 0.3 & & 1.74 & 0.22 & & 3.2 & 0.04 & & 3.2 & -0.2 \\
\hline 7 & & 1.37 & 0.56 & & 2.89 & 0.49 & & HS & 0.4 & & HS & 0.23 \\
\hline 9 & & 1.73 & 0.69 & & HS & 0.66 & & HS & 0.61 & & HS & 0.48 \\
\hline
\end{tabular}

Table.5. 1. The $\mathrm{n}$ value estimated with OD density test with Buffer / Hexadecane liquid partitioning OD remaining from Spectrophotometer test for NCYC-1324, NCYC-1681 and P.F bacteria. HS is saturated to the hydrophilic side of reaction equilibrium. P.F bacteria is not tested in $\mathrm{pH} 4$, therefore all marked as NA at pH4.

The number $\mathrm{n}$ (the multiple of standard deviation) was estimated with the model developed in section.3.3.4 based on the Gaussian distribution, as shown in Table.5.1. It was significant that NCYC-1324 was approximately saturated in all buffers from that observed in the OD curves (Figure.5.8 and 5.9). The NCYC-1324 yeasts surface 
hydrophilic chemicals had a higher density on the surface to provide drag force that provided more attraction to contain the yeast cells in the aqueous buffer solutions. Reaction equilibrium was saturated to hydrophilic for NCYC-1324 in buffers and not influenced by the application of different buffer $\mathrm{pH}$. Therefore, the $\mathrm{n}$ values were not calculated from the measurements on NCYC-1324 yeasts, as well as the NCYC-1681 yeasts in low ionic strengths and high $\mathrm{pH}$.

P.F bacteria measurements showed that the $\mathrm{n}$ value was closer to the mean centre $\left(\mathrm{C}_{50 \%}\right)$ of the Gaussian distribution. From the principle of Gaussian distribution, far from the mean value means higher sensitivity to value change. Thus, the error that existed in the $\mathrm{n}$ value calculated from the OD measured on P.F bacteria, as smaller compared with that calculated on yeast cells.

\subsection{Determination and Minimization of Error in Gaussian Distribution Data for Model Values Calculation}

In the model developed in section.3.3.4, gradient $\mathrm{G}$ was plotted against reaction equilibrium constant $\left(\mathrm{K}_{\mathrm{E}}\right)$ to estimate the mean value $\left(\mathrm{x}_{50 \%}\right)$ and standard deviation $(\delta \%)$ in the Gaussian distribution, as shown in Figure.3.30. The plotting of $\mathrm{G}$ to $\mathrm{K}_{\mathrm{E}}$ required a minimum of 3 sets of ODs remaining that measured different ionic strengths. Thus, only NCYC-1681 yeasts that had measurements at pH $4 \& 5$ and P.F bacteria at $\mathrm{pH}$ 5, $7 \& 9$ were qualified for the $\mathrm{K}_{\mathrm{E}}$ analysis. While, NCYC-1681 yeasts at other pHs and NCYC-1324 yeasts showed its saturation of hydrophilic groups, thus there were not enough data for the calculation. Figure.5.10 shows curves that were plotted with the raw estimation of the $n$ values calculated from the NCYC-1681 measurements (Figure.5.10a) and the P.F bacteria measurements (Figure.5.10b) in DI water, $0.001 \mathrm{M}, 0.01 \mathrm{M}$ and $0.1 \mathrm{M} \mathrm{NaCl}$ solutions at $\mathrm{pH}$. It was significant from Figure.5.10a that the $0.001 \mathrm{M} \mathrm{NaCl}$ solution and $\mathrm{DI}$ water buffer estimated the $\mathrm{n}$ value with a curve for NCYC-1681, which was parallel to the $\mathrm{X}$ axis, and tended to be parallel to other curves without an intersection point. 
In theory the plots of $\mathrm{G}$ against $\mathrm{K}_{\mathrm{E}}$ should intersect at the same value. However, Figure.5.10b shows that the curves plotted in different buffer concentrations intersect at different points far from each other; the curves plotted with $0.1 \mathrm{M}$ to $0.01 \mathrm{M}$ and $0.01 \mathrm{M}$ to $0.001 \mathrm{M} \mathrm{NaCl}$ solution buffers intersect at $\mathrm{K}_{\mathrm{E}}$ equals to 70.3 . Curves plotted with data from measurement in $0.1 \mathrm{M}$ and $0.01 \mathrm{M} \mathrm{NaCl}$ solution buffer do not cross any of the other two curves if $\mathrm{K}_{\mathrm{E}}$ was less than 126.

The values of $G$ were related to the value $n$, which could be calculated from the measured OD remaining and Gaussian distribution. Higher OD remaining would also represent a larger $n$ value and bring more sensitivity to the Gaussian plot, as stated in section.5.3.2.2.1. From the $\mathrm{n}$ values that were calculated and displayed in Table.5.1, $\mathrm{n}$ value estimated with NCYC-1681 yeast in DI water buffer and P.F bacteria in $0.1 \mathrm{M}$ $\mathrm{NaCl}$ solution buffer were regarded as the values with the largest error. So these were corrected to create a line that intersected at the same point as the other lines (this procedure was introduced in model development in section.3.3.4).

The reaction equilibrium constant was calculated without using the $\mathrm{n}$ values with the largest error, as shown in Figure.5.11a as an example based on P.F bacteria at pH5. The $\mathrm{n}$ value for $0.1 \mathrm{M} \mathrm{NaCl}$ solution buffer was adjusted by changing the highest $\mathrm{n}$ value with the intersection point found with low $\mathrm{n}$ values, as shown in the example plot of Figure.5.11b with P.F bacteria measured in $\mathrm{pH}$. In this adjustment, the $\mathrm{n}$ value of bacteria in $0.1 \mathrm{M}$ and $\mathrm{pH} 5 \mathrm{NaCl}$ solution were adjusted to 0.25 from its calculated value of 0.3 . The adjusted $n$ value of all the 5 groups of plotting were found and compared to the calculated $\mathrm{n}$ value in Table.5.2.

The reaction equilibrium was calculated from the adjustment intersections that were shown in Figure.5.11a. The adjusted $\mathrm{n}$ values were used to provide more accurate distribution information for cells in the buffer solutions with the highest error (NCYC-1681 in DI water buffers and P.F bacteria in $0.1 \mathrm{M} \mathrm{NaCl}$ buffers). With the adjusted $\mathrm{n}$ values, more accurate hydrophobicity values were calculated when calculating the Gaussian distribution information of mean value ( $\left.\mathrm{x}_{50 \%}\right)$ and standard deviation $(\delta \%)$, which is introduced in the following section. 

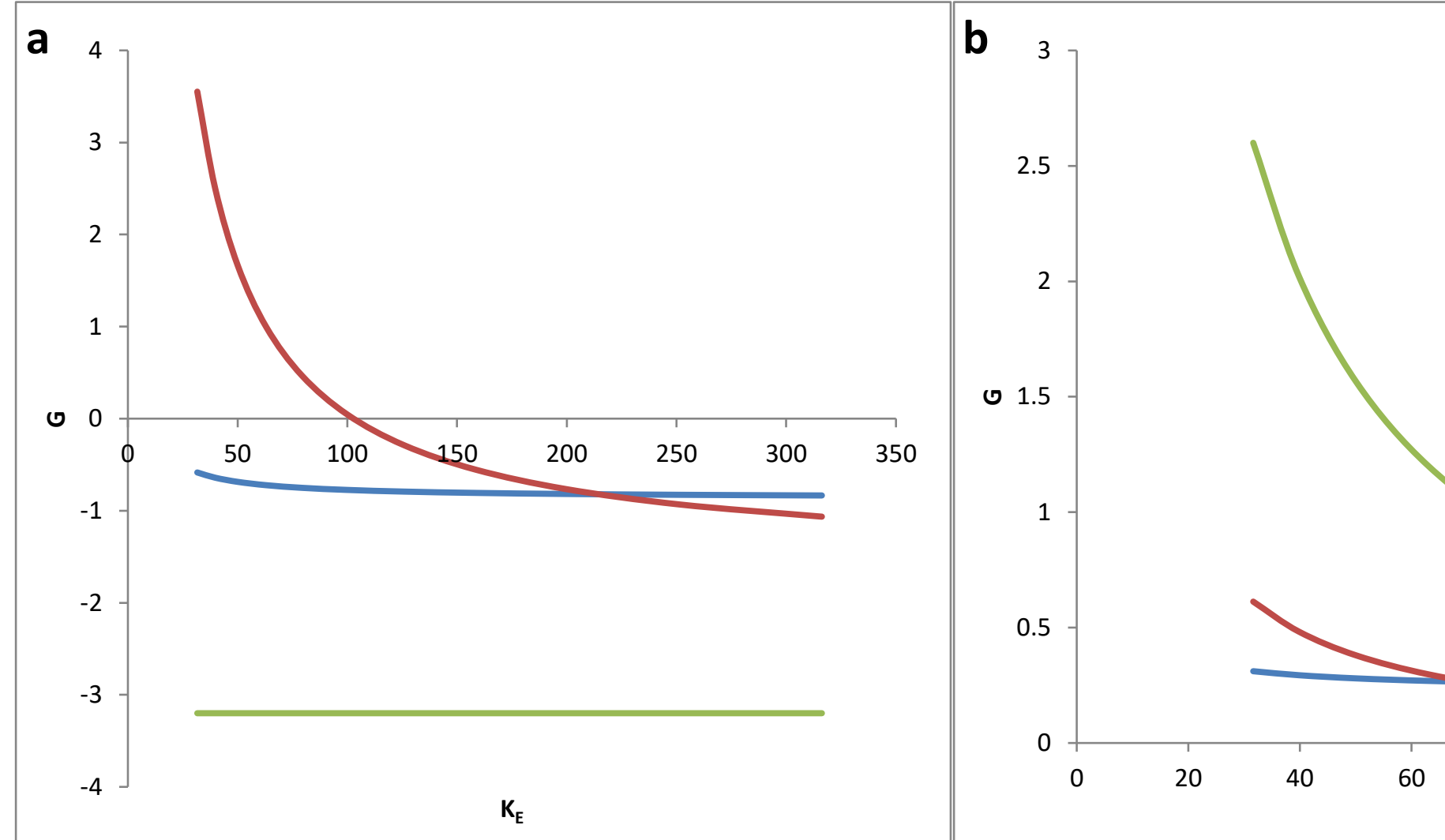

Figure.5. 10. G vs $\mathrm{K}_{\mathrm{E}}$ plot of NCYC-1681 (a) and P.F bacteria (b) in pH 5 buffer solutions $(0.1 \mathrm{M}, 0.01 \mathrm{M}, 0.0$ $\mathrm{x}$ axis as $\mathrm{K}_{\mathrm{E}}$ and $\mathrm{G}$ on $\mathrm{y}$ axis, curves is in comparison buffer concentration of blue (a) \& green (b) $=0.1 \mathrm{M}$ $0.001 \mathrm{M}$ and green $(\mathrm{b}) \&$ green $(\mathrm{a})=0.001 \mathrm{M}$ to DI water. 

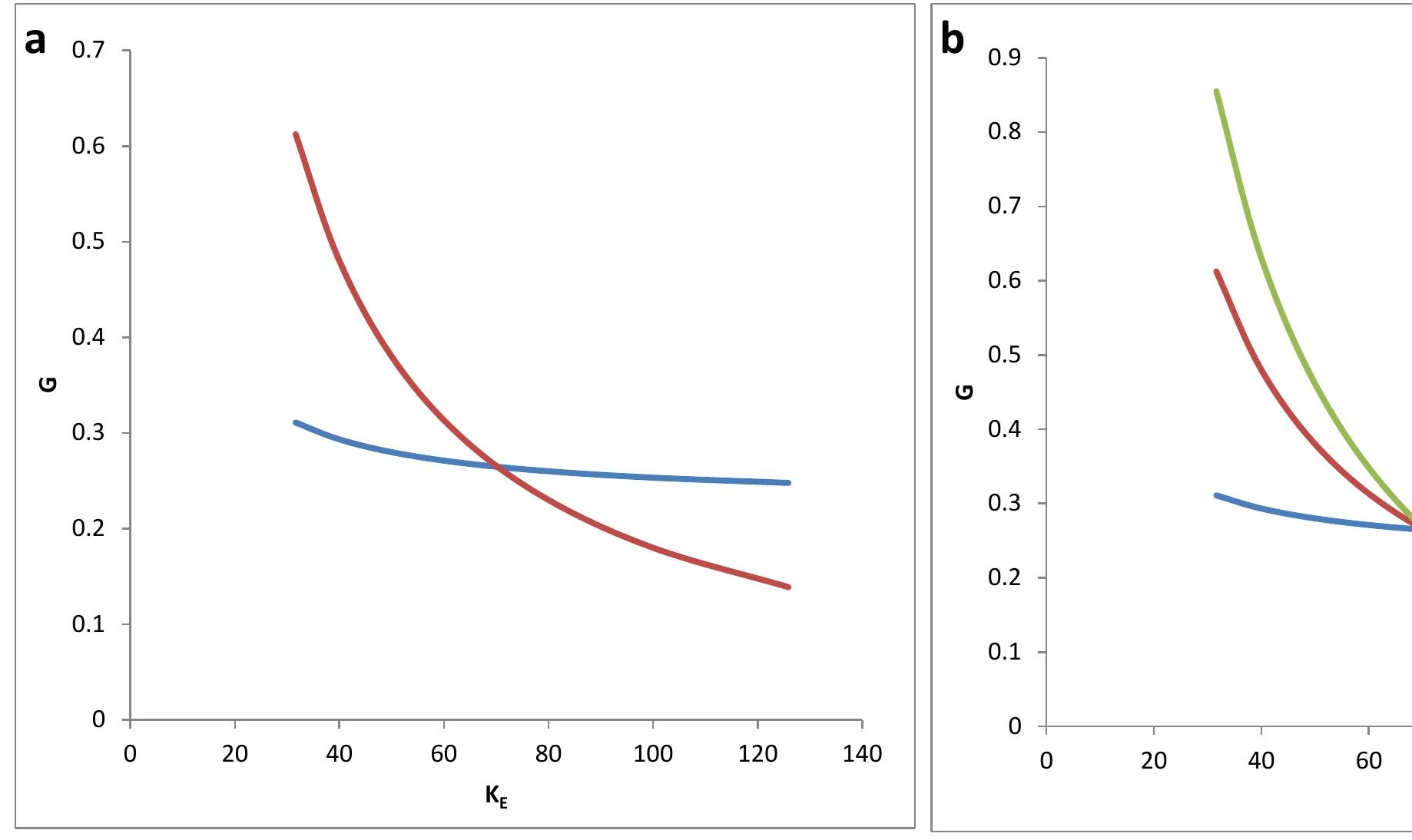

Figure.5. 11. $\mathrm{G}$ vs $\mathrm{K}_{\mathrm{E}}$ plot of P.F bacteria in $\mathrm{pH} 5$ buffers. $(\mathrm{a}$,$) is for \mathrm{K}_{\mathrm{E}}$ estimation plot only with the concentration (without the curve plot based on $\mathrm{n}$ values from cells in $0.1 \mathrm{M}$ and $0.01 \mathrm{M} \mathrm{NaCl}$ solution), a adjusted $\mathrm{n}$ values from cells in $0.1 \mathrm{M} \mathrm{NaCl}$ solution buffer. $\mathrm{X}$ axis as $\mathrm{K}_{\mathrm{E}}$ and $\mathrm{G}$ on $\mathrm{Y}$ axis, curves is in compa $0.001 \mathrm{M}$ to DI water, red $=0.01 \mathrm{M}$ to $0.001 \mathrm{M}$ and green $=0.1 \mathrm{M}$ to $0.01 \mathrm{M}$. 


\begin{tabular}{|c|c|c|c|c|c|}
\hline \multicolumn{7}{|c|}{ NCYC-1681 } \\
\hline \multirow{2}{*}{ Buffer Log C } & -1 & -2 & -3 & DI \\
\hline \multirow{3}{*}{ pH4 } & Calculated & 0.57 & 1.11 & 1.67 & 1.66 \\
\cline { 2 - 6 } & adjusted & 0.57 & 1.11 & 1.67 & 1.80 \\
\hline \multirow{3}{*}{ pH5 } & Calculated & 0.95 & 1.74 & 3.20 & 3.20 \\
\cline { 2 - 7 } & adjusted & 0.95 & 1.74 & 3.20 & 3.65 \\
\hline \multirow{3}{*}{ P.F pH5 } & Calculated & 0.30 & 0.22 & 0.04 & -0.2 \\
\cline { 2 - 7 } & adjusted & 0.25 & 0.22 & 0.04 & -0.2 \\
\hline \multirow{3}{*}{ P.F pH7 } & Calculated & 0.56 & 0.49 & 0.40 & 0.23 \\
\cline { 2 - 6 } & adjusted & 0.50 & 0.49 & 0.40 & 0.23 \\
\hline \multirow{2}{*}{ P.F pH9 } & Calculated & 0.69 & 0.66 & 0.61 & 0.48 \\
\cline { 2 - 6 } & adjusted & 0.67 & 0.66 & 0.61 & 0.48 \\
\hline
\end{tabular}

Table.5. 2. Comparison of calculated $n$ value and $K_{E}$ adjusted $n$ value for NCYC-1681.

\subsection{Calculation of the Chemical Group Distribution Which Influence Hydrophobicity}

With the reaction equilibrium constant $\left(\mathrm{K}_{\mathrm{E}}\right)$ already calculated, the mean value $\left(\mathrm{x}_{50 \%}\right)$, standard deviation $\left(\delta_{\%}\right)$ and adjusted non-reacted hydrophilic chemicals percentage (x) were then calculated and shown in Table.5.3 as well.

From Table.5.3, the $\mathrm{x}$ values calculated with the adjusted $\mathrm{n}$ values of NCYC-1681 in $\mathrm{pH} 4$ buffer were found to be larger than $100 \%$, while $\mathrm{x}$ should have been in the range of $0-100 \%$ based on the model development. Error might be induced from the approximate calculation of live cell rate, which was slightly different on different 
buffers. Thus, the oversized values were regarded as saturated as well.

\begin{tabular}{|c|c|c|c|c|}
\hline $\log C$ & -1 & -2 & -3 & DI \\
\hline \multicolumn{5}{|c|}{ NCYC-1681 pH4 } \\
\hline $\mathrm{K}_{\mathrm{E}}$ & \multicolumn{4}{|c|}{104.5} \\
\hline $\mathrm{X}_{50 \%}$ & \multicolumn{4}{|c|}{$-33.25 \%$} \\
\hline$\delta_{\%}$ & \multicolumn{4}{|c|}{$74.01 \%$} \\
\hline $\mathrm{x}$ & $8.95 \%$ & $49.32 \%$ & $91.17 \%$ & $100.61 \%$ \\
\hline \multicolumn{5}{|c|}{ NCYC-1681 pH5 } \\
\hline $\mathrm{K}_{\mathrm{E}}$ & \multicolumn{4}{|c|}{214.5} \\
\hline $\mathrm{X} 50 \%$ & \multicolumn{4}{|c|}{$-28.26 \%$} \\
\hline$\delta_{\%}$ & \multicolumn{4}{|c|}{$34.58 \%$} \\
\hline $\mathrm{x}$ & $4.66 \%$ & $32.27 \%$ & $83.04 \%$ & $98.65 \%$ \\
\hline \multicolumn{5}{|c|}{ P.F pH5 } \\
\hline $\mathrm{K}_{\mathrm{E}}$ & \multicolumn{4}{|c|}{70.3} \\
\hline $\mathrm{X} 50 \%$ & \multicolumn{4}{|c|}{$51.01 \%$} \\
\hline$\delta \%$ & \multicolumn{4}{|c|}{$192.70 \%$} \\
\hline $\mathrm{x}$ & $99.28 \%$ & $93.41 \%$ & $58.72 \%$ & $12.48 \%$ \\
\hline \multicolumn{5}{|c|}{ P.F pH7 } \\
\hline $\mathrm{K}_{\mathrm{E}}$ & \multicolumn{4}{|c|}{45.4} \\
\hline $\mathrm{X} 50 \%$ & \multicolumn{4}{|c|}{$-50.57 \%$} \\
\hline$\delta \%$ & \multicolumn{4}{|c|}{$298.47 \%$} \\
\hline $\mathrm{x}$ & $99.32 \%$ & $95.68 \%$ & $68.82 \%$ & $18.08 \%$ \\
\hline \multicolumn{5}{|c|}{ P.F pH9 } \\
\hline $\mathrm{K}_{\mathrm{E}}$ & \multicolumn{4}{|c|}{29.6} \\
\hline $\mathrm{X}_{50 \%}$ & \multicolumn{4}{|c|}{$-166.93 \%$} \\
\hline$\delta \%$ & \multicolumn{4}{|c|}{$400.04 \%$} \\
\hline $\mathrm{x}$ & $99.29 \%$ & $97.09 \%$ & $77.09 \%$ & $25.09 \%$ \\
\hline
\end{tabular}

Table.5. 3. $\mathrm{K}_{\mathrm{E}}, \mathrm{x}_{50} \%, \delta \%$ and $\mathrm{x}$ value for NCYC-1681 in $\mathrm{pH} 4 \& 5$ buffers and P.F 
bacteria in $\mathrm{pH} 5,7 \& 9$ buffers. $0.1 \mathrm{M}, 0.01 \mathrm{M}, 0.001 \mathrm{M} \mathrm{NaCl}$ solution and DI water were used as buffer.

The equilibrium constants, $\mathrm{x}_{50 \%}$ and $\delta_{\%}$ of $\mathrm{NCYC}-1681$ at $\mathrm{pH} 4$ and 5 were different to each other, as well as that calculated for P.F bacteria at $\mathrm{Ph} \mathrm{5,7}$ and 9 at different buffer $\mathrm{pH}$. Therefore, permanent change of the cell surface's chemicals were observed as theoretically estimated on cell surfaces. From the calculated $\mathrm{x}$ shown in Table.5.3, ionic strengths played a negative role on yeasts, which were hydrophilic at different buffer $\mathrm{pH}$. On the other hand, P.F bacteria were found to be more hydrophilic in higher ionic strengths at different $\mathrm{pH}$.

From the $\mathrm{x}_{50 \%}$ and $\delta_{\%}$ presented in Table.5.3, the $\mathrm{x}_{50 \%}$ values calculated from NCYC-1681 measurements were increasing with increase in $\mathrm{pH}$ and the $\mathrm{x}_{50 \%}$ values calculated from P.F bacteria measurements were decreasing with increase in $\mathrm{pH}$. Obviously, the role of buffer $\mathrm{pH}$ sensitive chemicals on NCYC-1681 and P.F bacteria cell surfaces hydrophobicity are different.

The $\delta \%$ calculated from yeasts measurements were decreasing with increase in $\mathrm{pH}$ and $\delta_{\%}$ calculated from P.F bacteria measurements were increasing with increase in $\mathrm{pH}$. Therefore, the higher $\mathrm{pH}$ would make the NCYC-1681 yeast cells become more sensitive to the reaction equilibrium change and P.F bacteria would be less sensitive to the equilibrium. From these phenomena of distribution change in different $\mathrm{pH}, \mathrm{pH}$ would change the cell surface chemical structures and be highly influential to the density of cell surface reactive chemicals. Ionic strengths would influence the surface chemistries through reaction equilibrium. There were also probably some hydrophilic chemistries that were not affected by ionic strength, which contributed to the change of $\mathrm{x}_{50 \%}$ and made NCYC-1324 saturated to hydrophilic in any buffer.

\subsection{Analysis of Relationship of the Cell Surface Hydrophobicity and Cell Surface Interactions}

From the analysis in section.5.3.2.2.3, the cell surface hydrophobicity was related to its surface chemical structures. Compared to the description in section.3.3.2, the 
influence of ionic strengths on cell surfaces hydrophobicity also changed the cell surface electrostatic interactions properties. The reaction equilibrium might make the cell surfaces charged ions change in different ionic strengths. From the reaction equilibrium, increase of buffer ionic strengths promoted the equilibrium to a balance with a higher proportion of reacted chemistries and the influence of increasing ionic strengths became less significant when ionic strengths were high. Compared to the zeta potentials measured with Zeta-Sizer in section.5.3.1.2, the zeta potentials were becoming less negative in higher ionic strengths and the rate of reduction of zeta potential tended to be smaller in higher ionic strengths. Higher $\mathrm{pH}$ was making the zeta potential of all the 3 kinds of cells to be more negative. As found in section.5.3.2.2.2 and 3, the hydrophobicity change on yeasts and bacteria at different $\mathrm{pH}$ and ionic strength were different. Therefore, the same influences on surface electrostatics of yeasts and bacteria were induced from different surface reactions.

The 3 kinds of cells were all becoming more negatively charged in higher $\mathrm{pH}$. The increase in $\mathrm{pH}$ would promote the cells to be more hydrophilic and negatively charged. From the hydrophobicity model calculation of standard deviation $(\delta \%)$, hydrophobicity of NCYC-1681 yeasts became more sensitive to reaction equilibrium with the $\mathrm{pH}$ increasing from 4 to 5 and P.F bacteria tending to be less sensitive to the equilibrium with the $\mathrm{pH}$ increasing from 5 to 9. Comparison of the Zeta-Sizer measurements on NCYC-1681 yeasts and P.F bacteria (Figure.5.3b and c) in different buffer ionic strengths, NCYC-1681 yeast zeta potential reduction rates were increasing from $\mathrm{pH} 4$ to $\mathrm{pH} 7$ and $\mathrm{P} . \mathrm{F}$ bacteria zeta potential reduction rates were decreasing from $\mathrm{pH} 5$ to $\mathrm{pH} 9$.

P.F bacteria were becoming more hydrophilic with increase in ionic strengths, while becoming less negatively charged at higher buffer ionic strengths. Theoretically, the reaction equilibrium was releasing hydrophilic groups or blocking hydrophobic groups on the cell surfaces and negatively charged groups were reducing in number with the adsorption of positive charged ions or blocking of surface negatively charged groups. Therefore, the hydrophilic chemicals on P.F bacteria cell surfaces was promoting the surface electrostatic interactions to be more positively charged. 
Different from the P.F bacteria, NCYC-1681 yeasts were becoming more hydrophobic with increase in ionic strengths and higher buffer concentrations made the bacteria less negatively charged. The reaction equilibrium was blocking the hydrophilic groups or increasing the hydrophobic groups' density on the cell surface, which would contribute to the cell surface negative charge as well.

Hydrophobicity of NCYC-1324 yeasts were not significantly changed in different buffer ionic strengths and less negative charged cell surface were induced by higher ionic strengths. The relationship of the electrostatic interactions and hydrophobicity was not able to be analysed; it could occur due to the richness of non-reactive hydrophilic chemistries on the cell surfaces. From the analysis of zeta potentials measured with the Zeta-Sizer and compared to the other two kinds of cells, these reactive chemistries were still significantly influencing the electrostatic interactions on cell surfaces, but its influence on hydrophobicity was weakened by non-reactive chemicals.

From the analysis and comparison of Zeta-Sizer and OD measurements, zeta potentials and hydrophobicity on cell surfaces were controlled by the surface chemical changes from the hydrolysis or adsorption of different charged ions or chemical groups.

\subsection{Imaging and Force Measurement of Cells Immobilized Membrane Surface}

AFM contact mode imaging on cell sample surfaces can provide detailed topography information of immobilised cell surfaces. Colloid probes were used in contact mode imaging to provide topography images. As described in detail in the following subsections, this section focused on the analysis of topography images and force curves at immobilised cell surfaces. 


\subsubsection{Comparison of AFM Contact Mode Imaging with Colloid Probes and Contact Mode Cantilevers}

Two kinds of AFM tip systems, contact mode cantilevers and colloid probes were used in imaging that within aqueous environments. The immobilised cells on sample surfaces provided a different topography compared to membrane surfaces. Cells immobilised at membrane surfaces were measured as a protrusion that was a few hundred nm higher than the membrane surfaces, which may influence the imaging quality.

Images of cells scanned with colloid probes are shown in Figure.5.12a (on NCYC-1324 yeast immobilised surfaces), Figure.5.12b (on NCYC-1681 immobilised surface) and Figure.5.12c (on P.F bacteria immobilised surface). Contact mode cantilevers were applied to imaging the sample surfaces with NCYC-1324 (Figure.5.12d), NCYC-1681 (Figure.5.12e) and immobilised P.F bacteria (Figure.5.12f). It showed that the effect of the size of colloid probes on accuracy of imaging was not significant. The imaging obtained from colloid probes showed the cells that were immobilised on surfaces clearly. Scrapes were found in imaging with both colloid probes and contact mode cantilevers; there were imaging artefacts that might be caused by frictions or collisions on the sample surfaces. Therefore, lower scanning speed as described in section.2.2.5.3 was used during AFM imaging to prevent the frictions or collisions that become too high, which might compromise the imaging.

The images clearly show the cells in Figure.5.12. Referring to the cell size study in section.5.3.1.1, yeast sizes were 3-6 $\mu \mathrm{m}$ (both NCYC-1324 and NCYC-1681) and P.F bacteria sizes were $1-2 \mu \mathrm{m}$. Both kinds of yeast cells showed a size of about $5 \mu \mathrm{m}$ and P.F bacteria were even smaller, about $2 \mu \mathrm{m}$. In Figure.5.12 a, d and Figure.5.12b, e, Cyclopore membrane surfaces were able to capture yeast cells. Cells at mean sizes of NCYC-1324 and NCYC-1681 were found, and smaller sizes were also observed. Figure.5.12c and $\mathrm{f}$ showed that there were not many P.F bacteria attached on the membrane surfaces. The size of P.F bacteria measured in this research were of 
comparable dimensions to the size and shape of the P.F bacteria measured by Fletcher in 1988 (Fletcher, 1988), the lighted spots found on images were found to be P.F bacteria. Some of the images surface structures such as flagellar were significantly compromised by contact mode scanning. It was significant that NCYC-1324 and NCYC-1681 yeast cells were more adhesive to Cyclopore membrane surfaces compared to P.F bacteria, as more yeast cells were immobilised on sample surfaces.
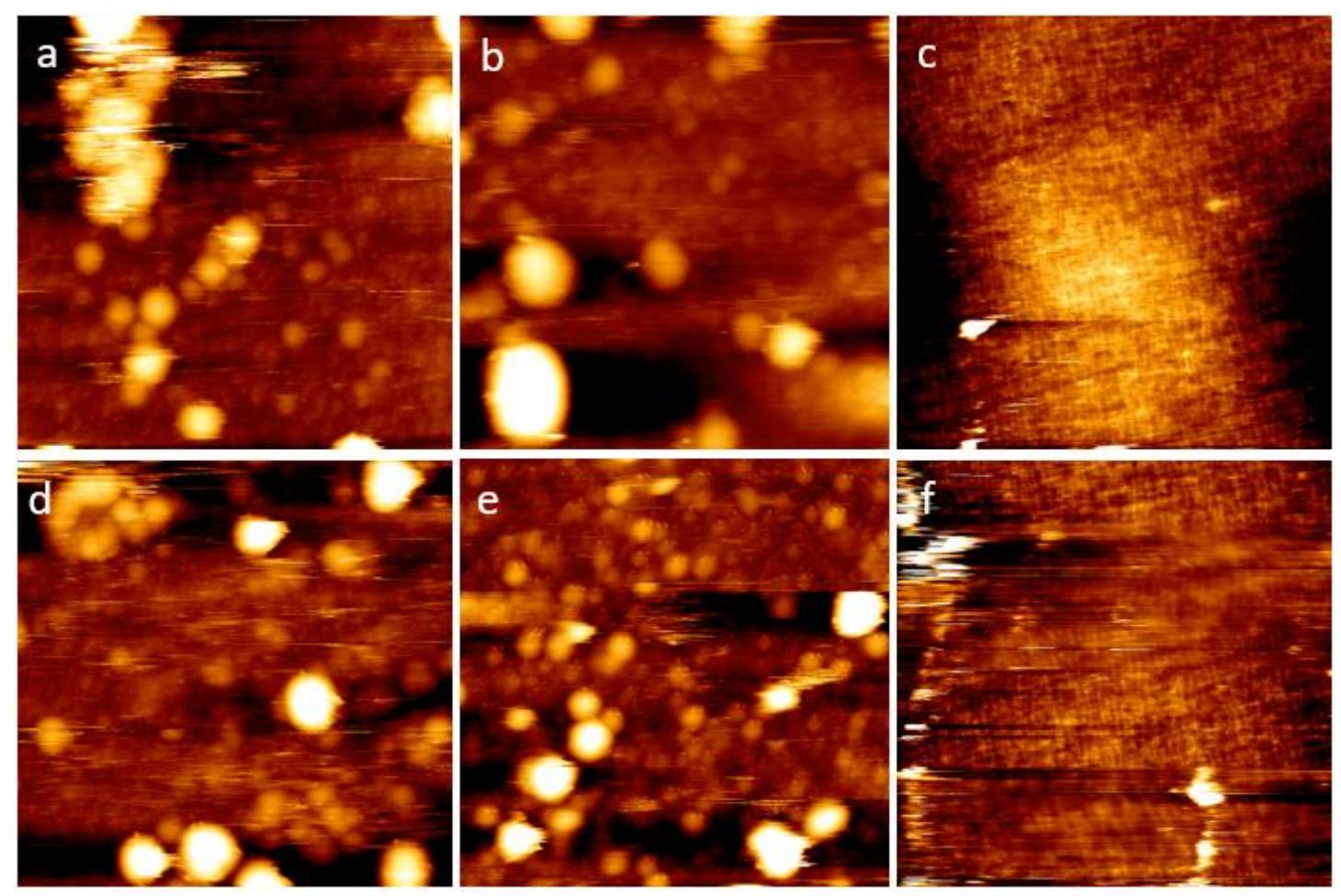

Figure.5. 12. Contact mode imaging of NCYC-1324 (a), NCYC-1681 (b) yeast and P.F bacteria (c) with silica colloid probe. Image is in $50 \mu \mathrm{m}$ scale and scanned in $1 \mathrm{~Hz}$. Image measured with silica bead colloid probe (size of 15.6 $\mu \mathrm{m}$ ). Contact tip (DNP-10) is also applied for surface imaging of NCYC-1324 (d), NCYC-1681 (e) and P.F bacteria (f).

In the comparison of yeasts imaging and bacteria imaging, there were many scrapes apparent within the images when the probe contacted with the cells. There were shadows around the larger cells because of the convolution between the surface and the cantilever tips or colloid probes. The contact areas of contact mode cantilevers were a few $\mu \mathrm{m}$, which would create a contact gap when contacting the edge of a convex structure. The shadows measured were significantly larger when scanned with colloid probes. It was because the cantilever tip contact surface was replaced with the 
colloid particles of larger size compared to the contact tips, which gave a larger and spherical contact surface.

The contact position of colloid probes was divided from the cell edge when cut into the gap region, as shown in Figure.5.13. Release of the bottom position would cause a shadow at the edge of the cell. From the analysis and that observed from the imaging, the shadows and scrapes on cell surfaces would not confuse identification of the cell position, but only reduce the quality of imaging.

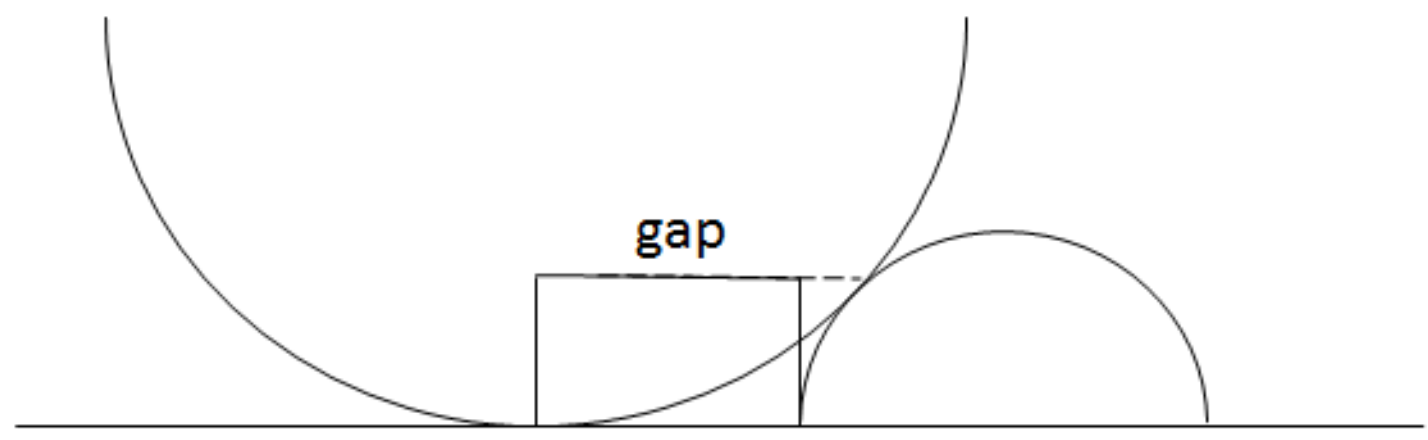

Figure.5. 13. Geometric illustration of the error applied in imaging of colloid probe used in contact mode imaging.

\subsubsection{Force Curves Measured on Cell Surfaces}

Force curves were measured on yeasts and P.F bacteria in pH5, 7 and 9 in $0.01 \mathrm{M}$ $\mathrm{NaCl}$ solutions. The examples of force curves were presented in Figure.5.14 for NCYC-1324 yeast cell, Figure.5.15 for NCYC-1681 yeast cell and Figure.5.16 for P.F bacteria in different buffer $\mathrm{pH}$. From the observation of Figure.5.14, 15 and 16, the adhesions were found to randomly occur, and multiple adhesion components were measured on retraction force curves from the 3 kinds of cells. Large adhesions were observed on the cell surfaces as well.

Electrostatic interactions were deconvolved from the approaching force curves at the contact points on the force curves. Therefore, the model that was developed in section.3.2.3 was employed to calculate the zeta potential on cell surfaces, the same as that used in section.4.3.2 on membrane surfaces. Then, the zeta potentials calculated 
were discussed in the following section.

Different from that measured on membrane surfaces, as shown in section.4.5.1, adhesions applied on cell-colloid interactions were with multiple components. Thus, the model that was developed in section.3.2.4 was applied to calculate the maximum adhesions and adhesion components and the adhesions were analysed in section.5.6.

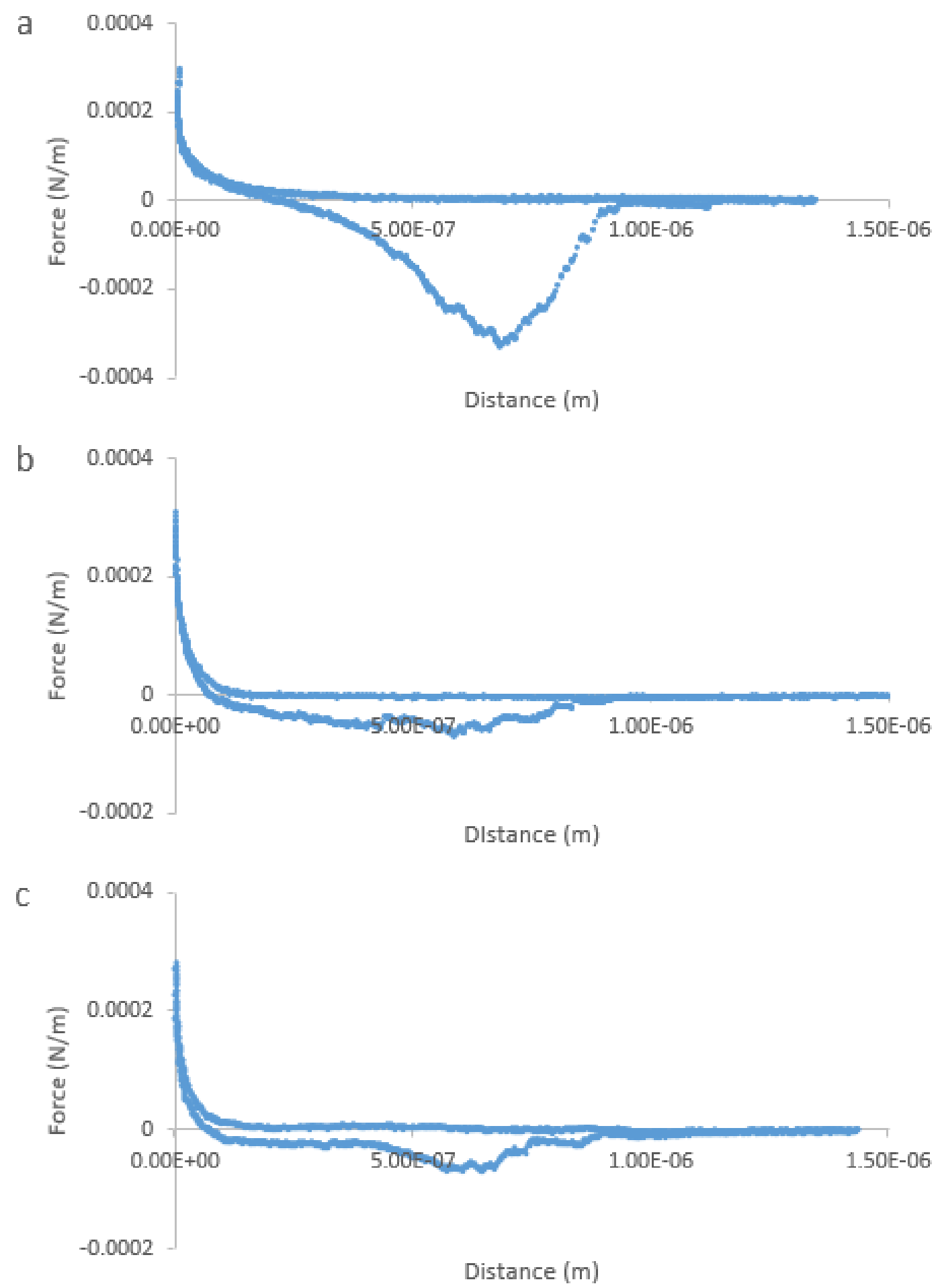


Figure.5. 14. Example of force curves measured on NCYC-1324 yeast cell in different $\mathrm{pH}$ and constant ionic strength, $\mathrm{a}$ is in $\mathrm{pH} 5, \mathrm{~b}$ is in $\mathrm{pH} 7$ and $\mathrm{c}$ is in $\mathrm{pH} 9$ buffer.
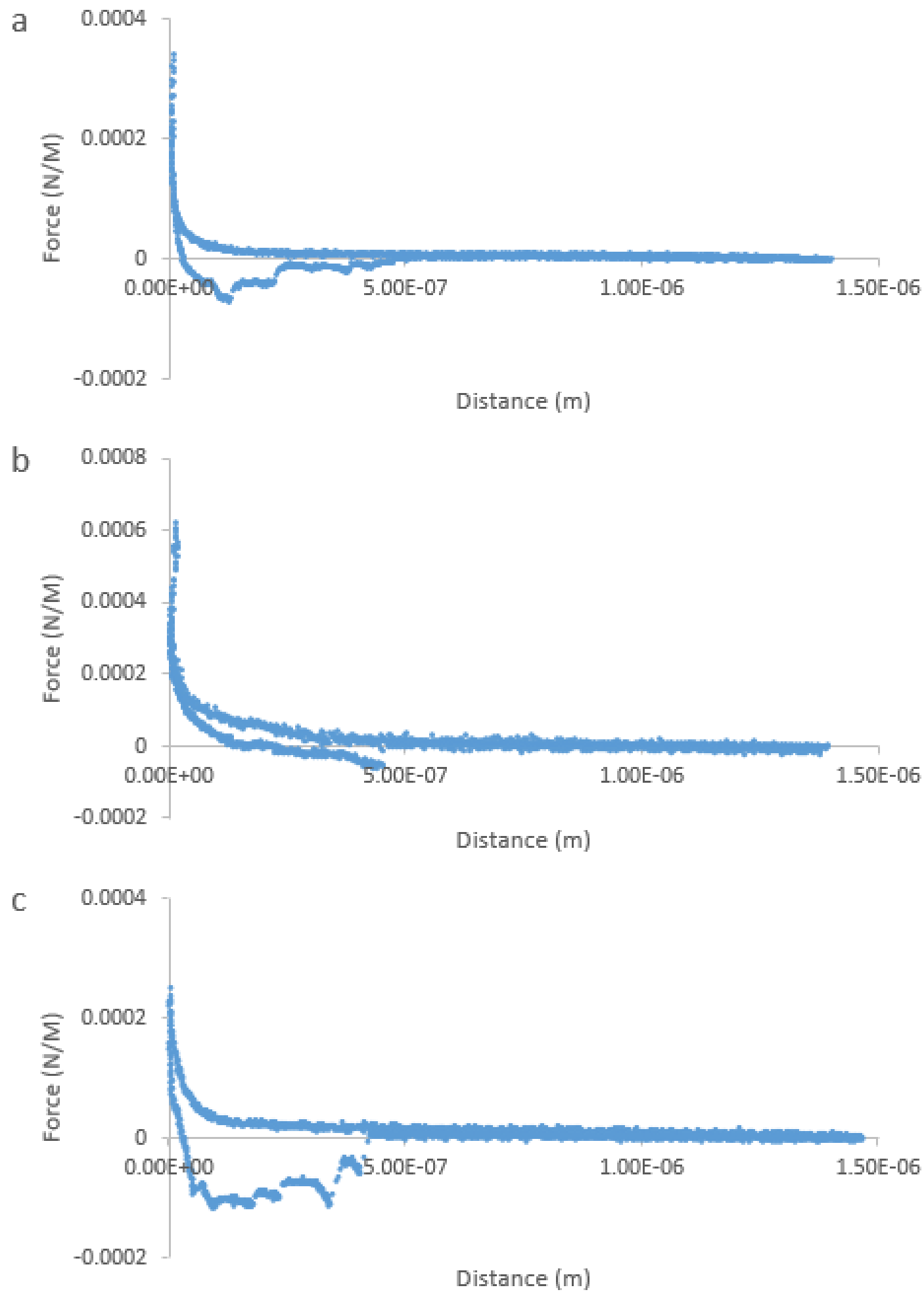

Figure.5. 15. Example of force curves measured on NCYC-1681 yeast cell in 
different $\mathrm{pH}$ and constant ionic strength, $\mathrm{a}$ is in $\mathrm{pH} 5, \mathrm{~b}$ is in $\mathrm{pH} 7$ and $\mathrm{c}$ is in $\mathrm{pH} 9$ buffer.

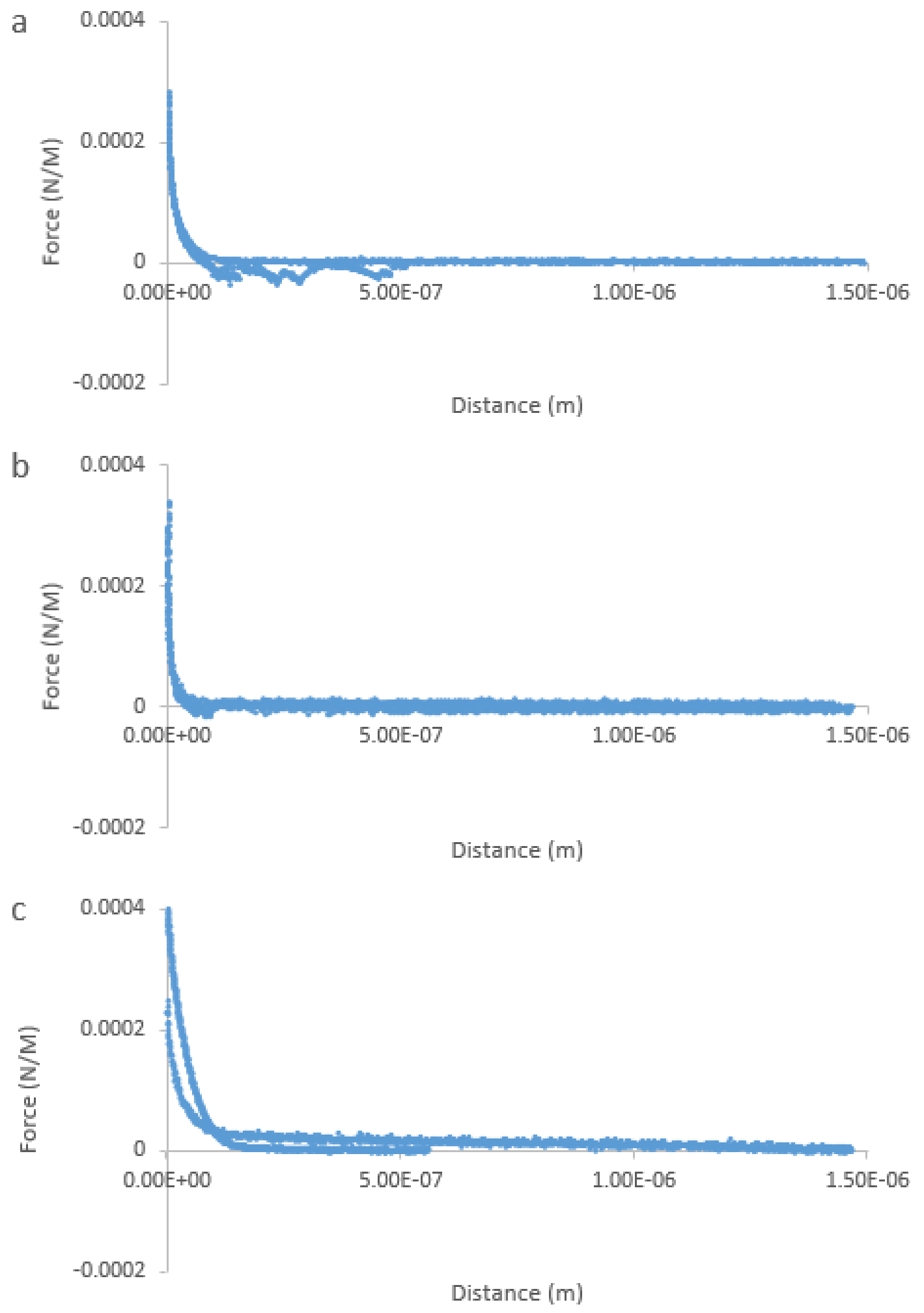

Figure.5. 16. Example of force curves measured on P.F bacteria in different $\mathrm{pH}$ and 
constant ionic strength, a is in $\mathrm{pH} 5, \mathrm{~b}$ is in $\mathrm{pH} 7$ and $\mathrm{c}$ is in $\mathrm{pH} 9$ buffer.

\subsection{Measurement of Cell Zeta Potential with AFM Force Spectroscopy using Silica and Hydroxyapatite Colloid probes}

Zeta potentials were calculated from the approaching force curves with the FORTRAN code that is shown in Appendix.2. Two kinds of colloid probes were used and the zeta potential of attached colloid particles of silica colloid probes (Figure.4.5) and hydroxyapatite colloid probes (Figure.5.17) were measured in buffers of different $\mathrm{pH}$ and ionic strengths. The probe surface charges were calculated from the colloids measured zeta potentials and used as a calculation basis in cell surface zeta potentials' calculation. The buffer effects on zeta potentials were analysed with the Man-Whitney U test (MWW), as showed in section.3.4.2.

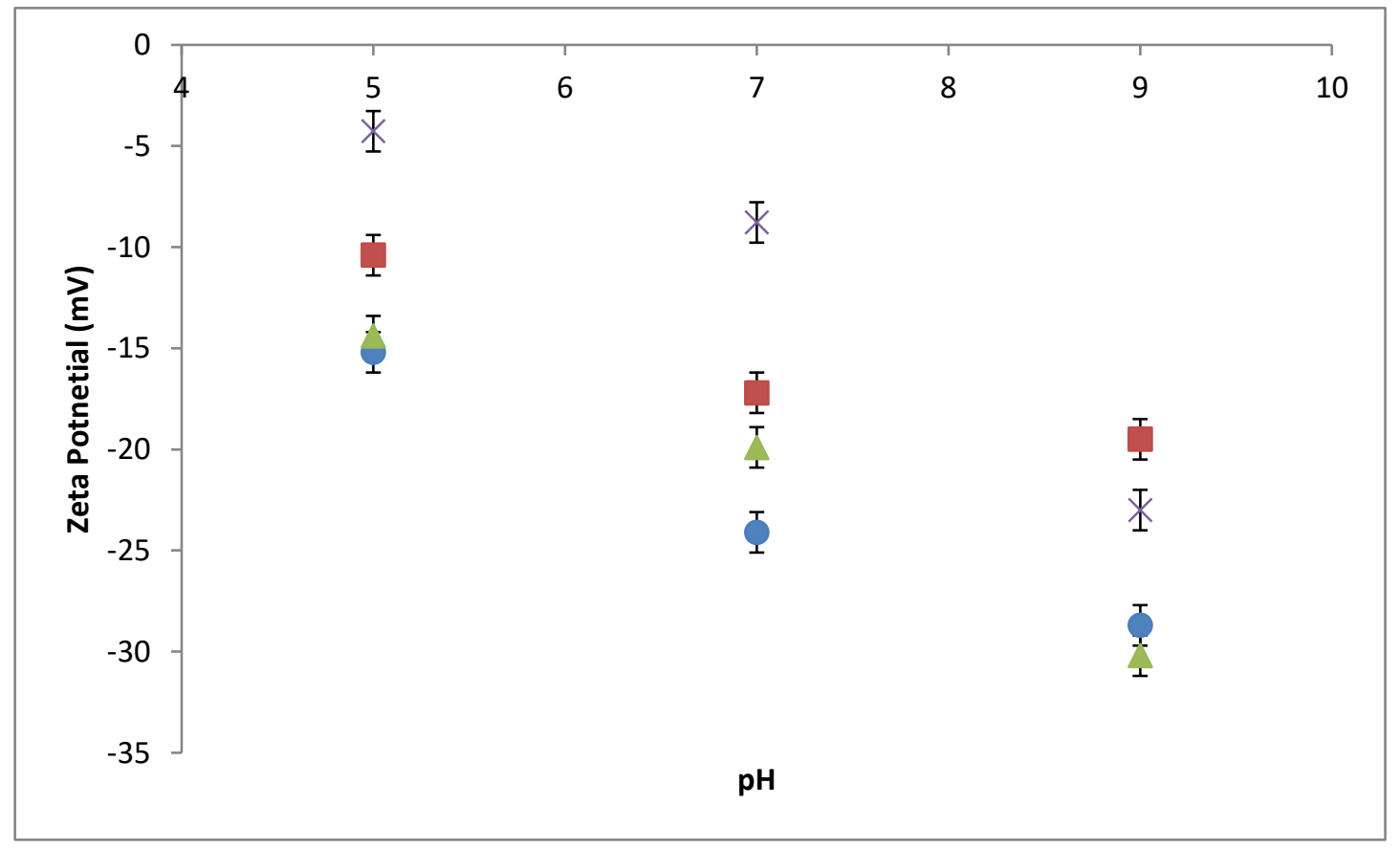

Figure.5. 17. Zeta potential measured on hydroxyapatite beads in different buffer $\mathrm{pH}$ and ionic strengths with zeta-sizer. Measurements are all in $\mathrm{NaCl}$ solutions or DI water. Purple cross is in DI water, read square is in $0.1 \mathrm{M}$, blue dot is in $0.01 \mathrm{M}$ and green triangle is in $0.001 \mathrm{M}$ of $\mathrm{NaCl}$ solutions. 
MWW comparison of the force curves calculated zeta potentials, showed there were significant differences in zeta potentials measured at cell surfaces in different buffer $\mathrm{pH}$. The distribution information of cell zeta potentials was calculated based on the Gaussian distribution (section.3.4.1) in different buffers. Then, the Gaussian distribution analysis was compared with the measured zeta potential to verify its feasibility in this study, as shown in Figure.5.18. From the comparison of zeta potential frequency calculated from force curves and the simulated Gaussian distribution, most measured zeta potential frequencies were located around the Gaussian distribution line, shown as the blue-dots in Figure.5.18. Only two of the distribution frequencies (black-triangle) were significantly different from the Gaussian distribution curve. The two frequencies were found located between $-27 \mathrm{mV}$ and $-33 \mathrm{mV}$, which deviated more than \pm 1 standard deviation from the average zeta potential calculated and were caused by low sample amount used in calculations. As discussed in section.3.4, the highly negative values measured were regarded as incidents that showed the possibility of heterogeneity far from its average. Potentially, it would improve with more force curves applied in calculations.

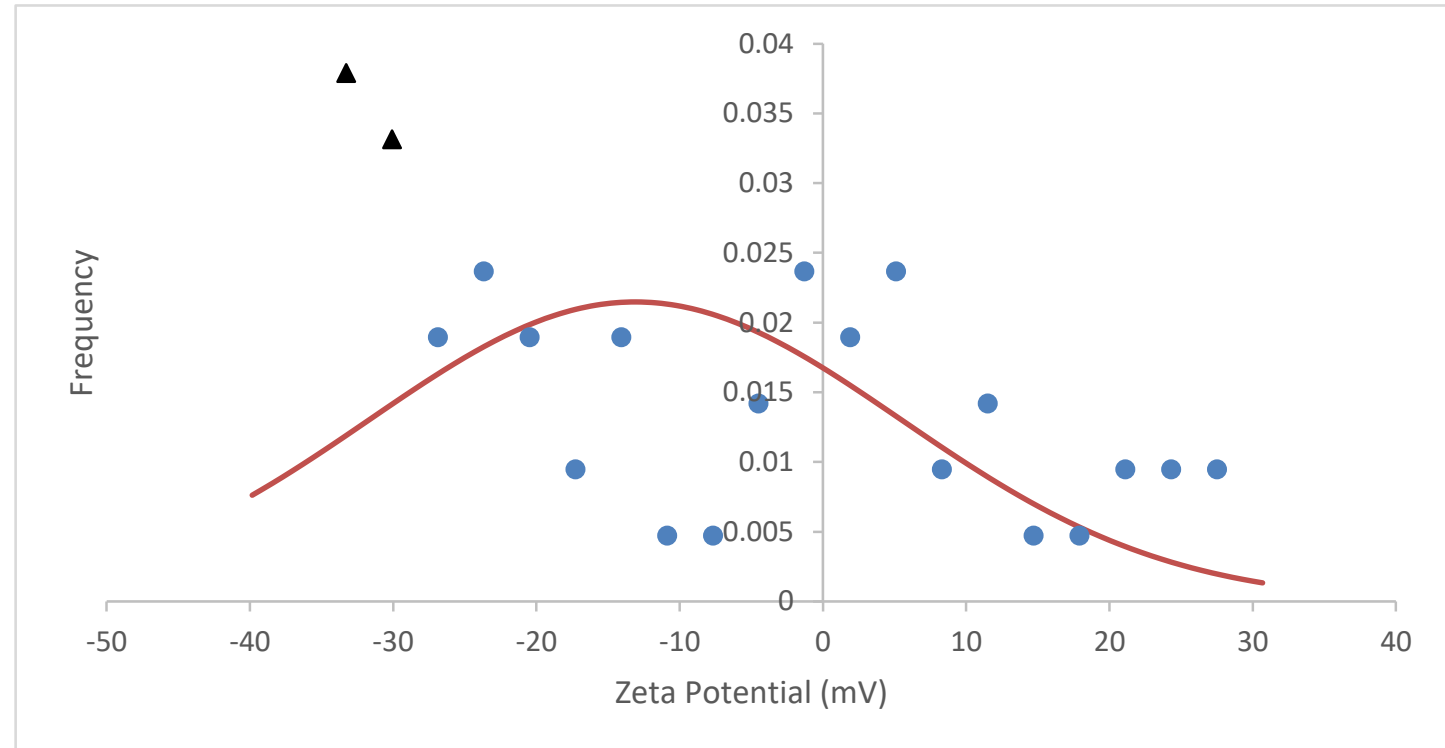

Figure.5. 18. Example comparison of frequency of calculated zeta potential (blue-dot) and its Gaussian distribution (red-line), real data regarded as with significant error were marked as black-triangle. Measurement based on NCYC-1681 cell in $0.01 \mathrm{M}$ $\mathrm{NaCl}$ pH5 buffer. 

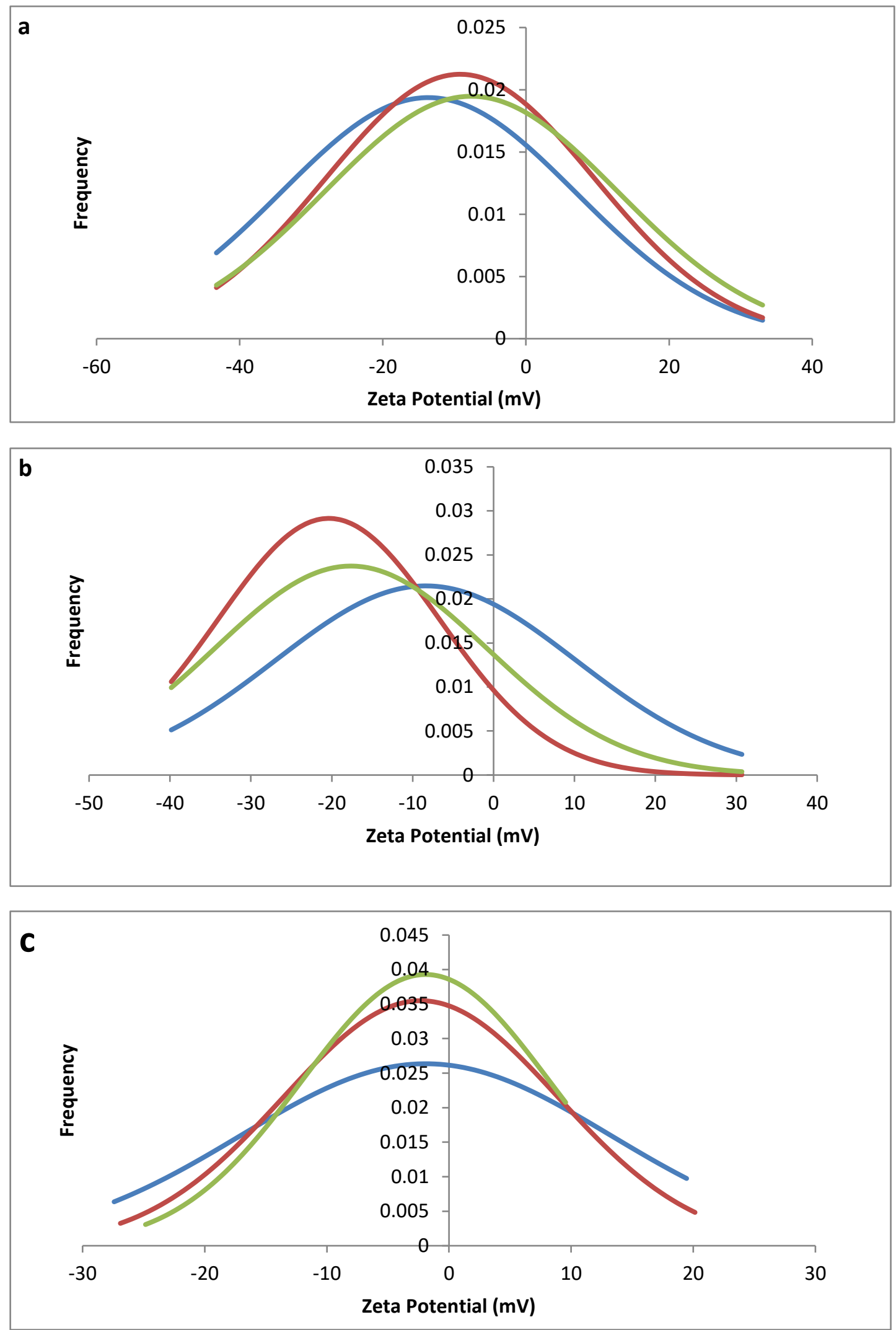

Figure.5. 19. Gaussian distribution estimation based on Gaussian distribution, a for NCYC-1324, b for NCYC-1681 and c for P.F bacteria. $\mathrm{pH} 5$ shows in blue, $\mathrm{pH} 7$ is in red and green for $\mathrm{pH} 9$. Unit of zeta potential is in $\mathrm{V}$. 
The Gaussian distribution for measurement made at yeast and bacterial surfaces in different $\mathrm{pH}$ were plotted in Figure.5.19 for both kinds of cells. In Figure.5.19a, the average zeta potentials of $\mathrm{NCYC}-1324$ were $-13.6 \mathrm{mV}$ at $\mathrm{pH} 5,-9.2 \mathrm{mV}$ at $\mathrm{pH} 7$ and $-7.7 \mathrm{mV}$ at $\mathrm{pH}$. Compared with the Zeta-Sizer measured results in section.5.3.1.2, the calculated zeta potential range was close to zeta potential of around $-10 \mathrm{mV}$ measured from Zeta-Sizer at all $\mathrm{pH}$. Another significant difference was that the Zeta-Sizer measured average values were becoming more negative with increase in $\mathrm{pH}$. The standard deviations of AFM force curves that calculated zeta potential distribution were found to be about $\pm 20 \mathrm{mV}$ in all different $\mathrm{pH}$ conditions. The differences between calculated result based on force curves and Zeta-Sizer measurement were less than $\pm 5 \mathrm{mV}$, which was a tolerable difference compared to the standard deviation.

Figure.5.19b shows the zeta potential distribution of NCYC-1681 calculated from AFM force curves in different $\mathrm{pH}$ conditions. The average zeta potentials calculated were found to be $-8.4 \mathrm{mV}$ in $\mathrm{pH} 5,-20.4 \mathrm{mV}$ in $\mathrm{pH} 7$ and $-17.6 \mathrm{mV}$ in $\mathrm{pH} 9$. Compared to results that were measured from Zeta-Sizer, which was $-13.1 \mathrm{mV}$ in $\mathrm{pH} 5,-17.6 \mathrm{mV}$ in $\mathrm{pH} 7$ and $-22.5 \mathrm{mV}$ in $\mathrm{pH} 9$, the data calculated from force curves contained an error of less than $\pm 5 \mathrm{mV}$. Standard deviations of the calculated distribution were between $\pm 13 \mathrm{mV}$ and $\pm 20 \mathrm{mV}$ in different $\mathrm{pH}$ conditions, which made the average zeta potentials measured from Zeta-Sizer, located in the range of zeta potential distribution calculated from the force curves.

Figure.5.19c shows the zeta potential distribution on P.F bacteria calculated from force curves in different $\mathrm{pH}$. The calculated average zeta potentials were found to be $-1.9 \mathrm{mV}$ in $\mathrm{pH} 5,-2.3 \mathrm{mV}$ in $\mathrm{pH} 7$ and $-1.9 \mathrm{mV}$ in $\mathrm{pH}$. Compared with the zeta potential measured with Zeta-Sizer, which was from $-7 \mathrm{mV}$ to $-8 \mathrm{mV}$ in the measured $\mathrm{pH}$ conditions, the differences were less than $\pm 6 \mathrm{mV}$. Both results showed that the P.F bacteria zeta potentials were not significantly influenced by the buffer $\mathrm{pH}$. Standard deviations were from $\pm 10 \mathrm{mV}$ to $\pm 15 \mathrm{mV}$ in different $\mathrm{pHs}$, thus the Zeta-Sizer measured results were in the range that were analysed from force curves. 
a
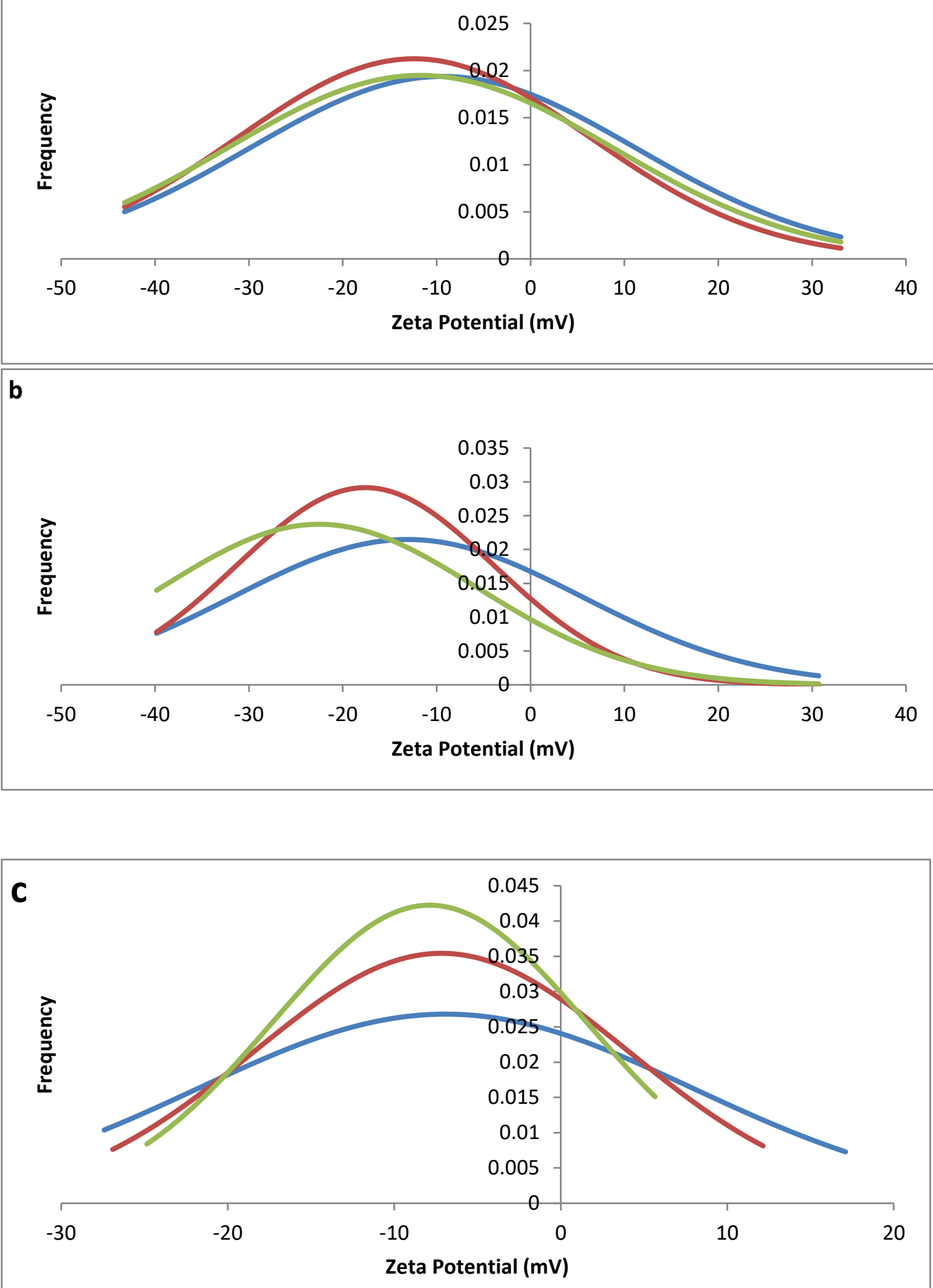

Figure.5. 20. Gaussian distribution estimation based on MWW model, data corrected with zeta-sizer results, a for NCYC-1324, b for NCYC-1681 and c for P.F bacteria. $\mathrm{pH} 5$ shows in blue, $\mathrm{pH} 7$ is in red and green for $\mathrm{pH} 9$. Unit of zeta potential is in V. 
The differences found between Zeta-Sizer measurements and force curve calculations were from $\pm 5 \mathrm{mV}$ to $\pm 6 \mathrm{mV}$. In all the calculated zeta distributions, there was a standard deviation of more than $\pm 10 \mathrm{mV}$ and less than $\pm 20 \mathrm{mV}$, which were still large but could only be optimised with the inclusion of a larger number of force curves in calculation. As discussed in section.3.4, more force curves would optimise the calculation of the average zeta potential for cell surfaces and would likely ensure the AFM results approached the average measured with the Zeta-Sizer.

With the discussion in section.5.3.2.2.4, the rate of change of zeta potential (Zeta-Sizer) with $\mathrm{pH}$ or ionic strength was influenced by hydrophobicity. The zeta potential value sensitivity was highly influenced by the Gaussian distribution $\left(\delta_{\%}\right)$ calculated for the hydrophobicity, which was also related to the $\mathrm{pH}$. The calculated results from the force curves were compared with the hydrophobicity analysis for P.F bacteria at different $\mathrm{pH}$, because both yeasts had surfaces that were too saturated to provide hydrophobicity analysis within buffers from $\mathrm{pH} 5$ to $\mathrm{pH} 9$ (as shown in Table.5.3). When the $\delta \%$ was calculated for P.F bacteria, the change in zeta potential was becoming less significant with the increasing $\mathrm{pH}$. Therefore, the standard deviation of zeta potential for bacteria also became smaller with increasing buffer $\mathrm{pH}$. The standard deviations for P.F bacteria AFM zeta potential calculation was found to be $\pm 15.1 \mathrm{mV}$ in $\mathrm{pH} 5, \pm 11.2$ in $\mathrm{pH} 7$ and $\pm 10.2 \mathrm{mV}$ in $\mathrm{pH}$. Therefore, zeta potentials are influenced by $\mathrm{pH}$, which is due to the $\mathrm{pH}$ influences on the cell surface chemistry.

The Zeta-Size instrument is a mature zeta potential measuring method, that can provide more accurate estimation on average cell zeta potential for a population of cells or particles, as introduced in section.3.4. All measurements from Zeta-Sizer were in the range of less than one standard deviation whereas the values calculated form AFM force curves had a much broader range. Theoretically, it is reasonable to assume that the measuring accuracy of the AFM force curves calculation is potentially as accurate as the with the Zeta-Sizer if enough force curves were analysed. Thus, a more accurate distribution was achieved by considering both measurement techniques; the AFM data were adjusted to achieve the same mean value as measured from the Zeta-Sizer but keeping the inherent heterogeneity as recorded by the standard 
deviations calculated from the force curves methods. For example, the adjusted distribution was plotted in Figure.5.20a for NCYC-1324, Figure.5.20b for NCYC-1681 and Figure.5.20c for P.F bacteria.

\subsection{AFM Adhesion Analysis of Cell Surfaces}

The adhesion that occurred in cell-colloid interactions were studied for NCYC-1324, NCYC-1681 yeasts and P.F bacteria in contact with two kinds of colloid probes; silica beads and hydroxyapatite beads probes. Adhesion was influenced by the surface chemical structures of the contact surfaces, as described in section.4.5.1. In this study, the calculation model was applied to analyse the maximum distance of adhesion interaction and the types of the adhesion forces that contributed to the cell-colloid interactions for yeast and bacterial surfaces.

\subsubsection{Maximum Adhesion and Adhesion Distance Analysis}

There was a broad range of cell surface adhesion measured with silica beads. Adhesions larger than 7 thousand $\mathrm{pN}$ and as small as $53 \mathrm{pN}$ were measured for all the 3 kinds of cells. For the detected adhesion interactions, the majority of the adhesions were located between $500 \mathrm{pN}$ and $3000 \mathrm{pN}$ for NCYC-1324 and NCYC-1681 yeasts in buffers of different $\mathrm{pH}$ (Table.5.4), while P.F bacteria had insignificant adhesion to silica colloid probes. As shown in Table.5.4, the adhesion forces measured on yeast silica contact had larger average minimum and maximum values for NCYC-1324 yeasts as compared to that measured for NCYC-1681 yeasts at different pH. P.F bacteria had no adhesion for the silica colloid probe in any of the buffers studied so no data is supplied in Table.5.4.

\begin{tabular}{|l|l|l|l|}
\hline $\mathrm{pH}$ & $\mathrm{pH} 5$ & $\mathrm{pH} 7$ & $\mathrm{pH} 9$ \\
\hline \multicolumn{3}{|c|}{ NCYC-1324 - Silica bead } \\
\hline
\end{tabular}




\begin{tabular}{|c|c|c|c|}
\hline Minimum & 372.257 & 706.4666 & 388.327 \\
\hline Maximum & 6736.718 & 7294.774 & 7037.501 \\
\hline Average & 3235.804 & 2306.142 & 2117 \\
\hline Count & 24 & 24 & 25 \\
\hline \multicolumn{4}{|c|}{ NCYC-1681 - Silica bead } \\
\hline Minimum & 154.0552 & 72.05967 & 317.8304 \\
\hline Maximum & 1275.339 & 5996.758 & 3420.727 \\
\hline Average & 458.1206 & 777.9159 & 1342.057 \\
\hline Count & 18 & 17 & 12 \\
\hline \multicolumn{4}{|c|}{ P.F bacteria - Hydroxyapatite bead } \\
\hline Minimum & 53.21418 & 88.30483 & 11251.79 \\
\hline Maximum & 656.5005 & 1036.318 & 11251.79 \\
\hline Average & 168.764 & 287.5879 & 11251.79 \\
\hline Count & 11 & 26 & 1 \\
\hline
\end{tabular}

Table.5. 4. Minimum, maximum, average adhesion force strength and number of force curves detected with adhesion force found in NCYC-1324, NCYC-1681 yeast cell - silica bead contact and P.F bacteria - hydroxyapatite bead contact.

There was larger adhesion forces for cell hydroxyapatite bead interactions. Maximum measured adhesion was as large as $100 \mathrm{nN}$ and most of the adhesions measured were larger than $10 \mathrm{nN}$ for both NCYC-1324 and NCYC-1681 yeasts. Small adhesions of less than $100 \mathrm{pN}$ were also found in retraction force curves measured at both yeasts. The large differences between maximum and minimum adhesion on hydroxyapatite-yeast contact made the average calculation difficult to present the adhesion statistically. Hence, this was not included in Table.5.4. However, it is significant that the adhesions measured on yeast cell surfaces were higher when contacted with hydroxyapatite colloid probes compared to that found in contact with silica colloid probes.

The adhesion of P.F bacteria - hydroxyapatite bead contacts were found to be significantly larger as compared with the P.F bacteria - silica bead contacts. As 
presented in Table.5.4, 11 adhesion force curves were found in $\mathrm{pH} 5$ and 26 were found in $\mathrm{pH} 7$. There was no significant adhesion in $\mathrm{pH} 9$; only one force curve was found with a significant adhesion force in $\mathrm{pH} 9$. The maximum adhesion force found was not as large as the adhesion for yeast cells $(656 \mathrm{pN}$ in $\mathrm{pH} 5$ and $1036 \mathrm{pN}$ in $\mathrm{pH} 7)$. The only measured adhesion force measured in $\mathrm{pH} 9$ is as large as $11251.8 \mathrm{pN}$; thus, it is reasonable to assume that large adhesion forces existed for P.F bacteria hydroxyapatite bead interactions as well.

Adhesion distances were calculated from the force curves. Long adhesion distances of more than $200 \mathrm{~nm}$ were found as well as short adhesion distances of around $10 \mathrm{~nm}$. The majority of the adhesion interactions measured were founded to be short-distance influencing adhesion. The long-distance influencing adhesions were mostly found with large adhesion strengths of a few $\mathrm{nN}$. Short-distance adhesions existed in both small and large adhesion strengths, from $100 \mathrm{pN}$ to $10 \mathrm{nN}$. Therefore, the surface chemistry that induced large adhesion strengths acted over long distances as opposed to surface chemistry that acted over short distance which induced either large or small adhesion forces.

The typical adhesions were classified and presented in Figure.5.21. Different kinds of typical large adhesion are presented in Figure.5.21a-c. Figure.5.21a presents a large adhesion with a relatively smooth break-off. The adhesion had a long influencing distance before probe separated from the cell. From the detected release from zero distance, the cell surface might be damaged because of the large adhesion force. Figure.5.21b shows a large adhesion with rapid break-off. The influencing distance was significantly smaller compared to that showed in Figure.5.21a. Figure.5.21c is a large adhesion with rapid break-off as well. The adhesion force curve was found to be fluctuating before it achieved maximum strength. However, adhesion components were applied, which gave a long-distance and smooth increase in force before it achieved the maximum adhesion force. From these large adhesions, it was observed that there was normally one adhesion component that could be measured from these adhesions, as it was found that the applying and breaking-off of the adhesion was smooth (Figure.5.21c). 
Typical small adhesion events that were observed are presented in Figure.5.21d-i. Figure.5.21d shows a force curve that had a short-distance adhesion and simply broke away with no fluctuation. Figure.5.21e, $\mathrm{f}$ and $\mathrm{g}$ are short distance adhesions with fluctuation during break off. Figure.5.21e shows adhesion with wave shape fluctuations when pulled off that quickly broke at a yield position. Figure.5.21f was adhesion with a partial breakaway and a smooth change in influence over a long distance. The long-distance effect of contact and then small and fluctuating adhesion is presented at Figure.5.21g. Figure.5.21h shows small adhesion with long distance and smooth release instead of sudden breakaway. Figure.5.21i also shows small adhesion with long distance release. Different from Figure.5.21h, the adhesion was released with fluctuations, which were caused by different adhesion events. From the observations on these force curves with small adhesion strength, it showed that the possibility of measuring a small or large adhesion event with a large or small interaction distance was random and that there was higher possibility that a small adhesion force would be measured on contact with a single cell immobilised at the surface.

From the adhesion analysis achieved from calculation and observed from force curves, adhesions were found to be applied on the cell-probe interactions with different adhesion strengths, fluctuations and influencing distances. Adhesions were found as large as $100 \mathrm{nN}$ and as small as $53 \mathrm{pN}$. Large adhesions had a longer influencing distance and the adhesion influencing distances were short or long when adhesion strengths were small. Fluctuations were mostly found on small adhesions, while it was measured on some of the large adhesions as well. The fluctuations observed in adhesion elements of force curves were regarded as being caused by different components applied in the adhesion. Application of different types of adhesion components was then calculated and analysed in the following section. 

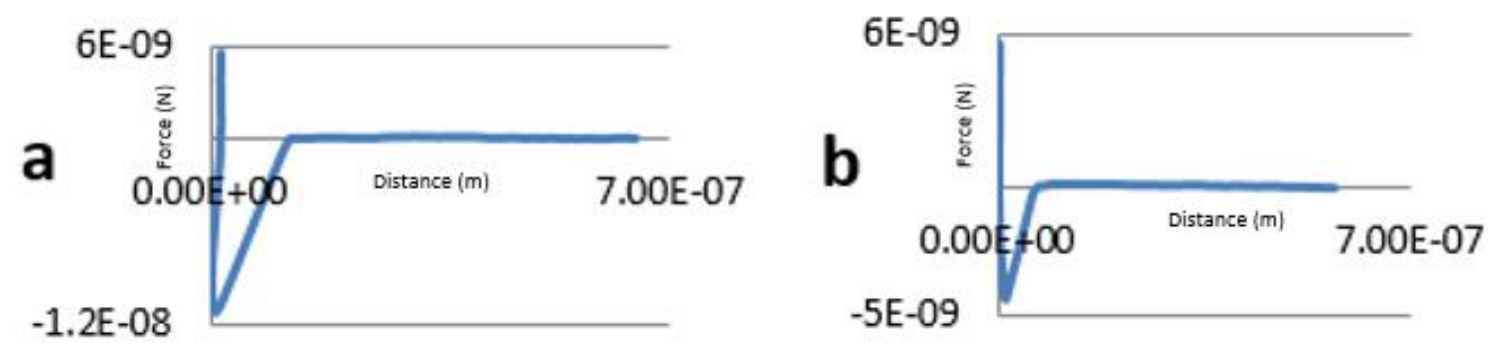

4.00E-09
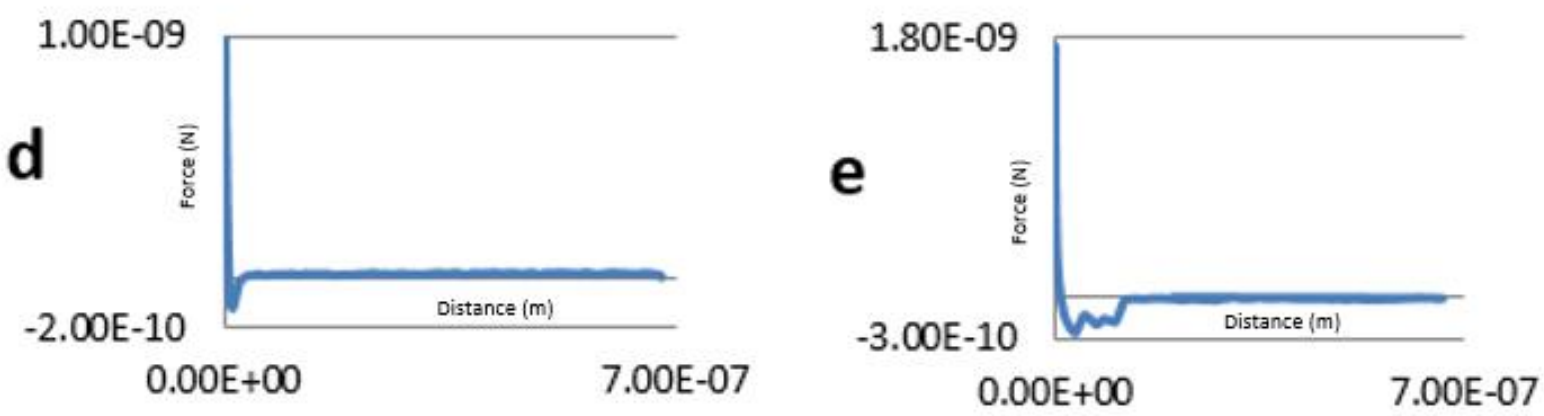

$1.50 \mathrm{E}-09$

$\mathbf{f}$

$-2.00 \mathrm{E}-10$

0.00
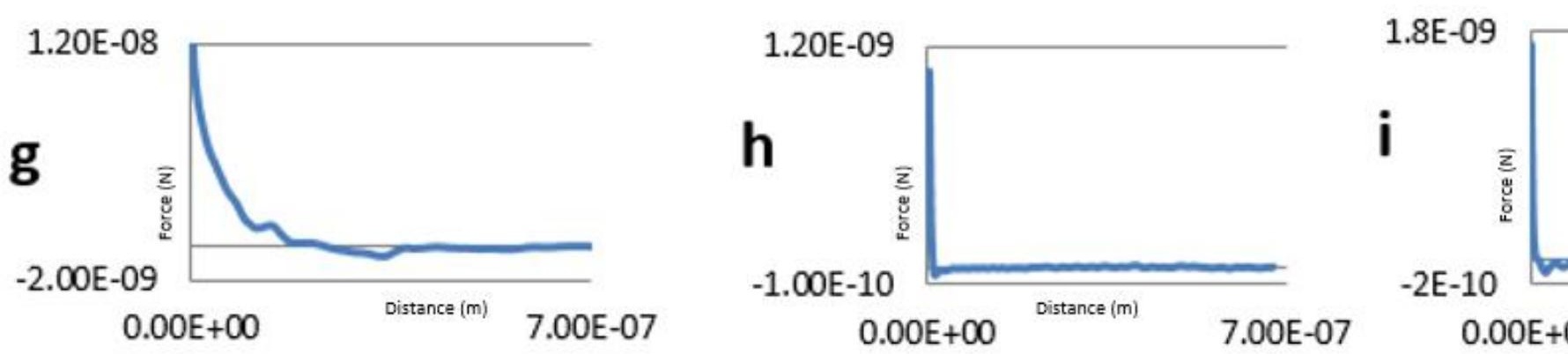

Figure.5. 21. Typical retraction force curve with adhesion detected. $\mathrm{X}$ axis is distance in meter; $\mathrm{Y}$ axis is force in nev 


\subsubsection{Adhesion Type Analysis}

The adhesion type calculated from AFM retraction force curves was provided by a count of how many kinds of adhesion components were applied, as discussed in section.3.2.4.2. Some of the significant components were observed in force curves, such as the force curves shown in Figure.5.21g. Adhesion components were counted from different cell-probe interactions and illustrated as percentages that were shown in Figure.5.22.

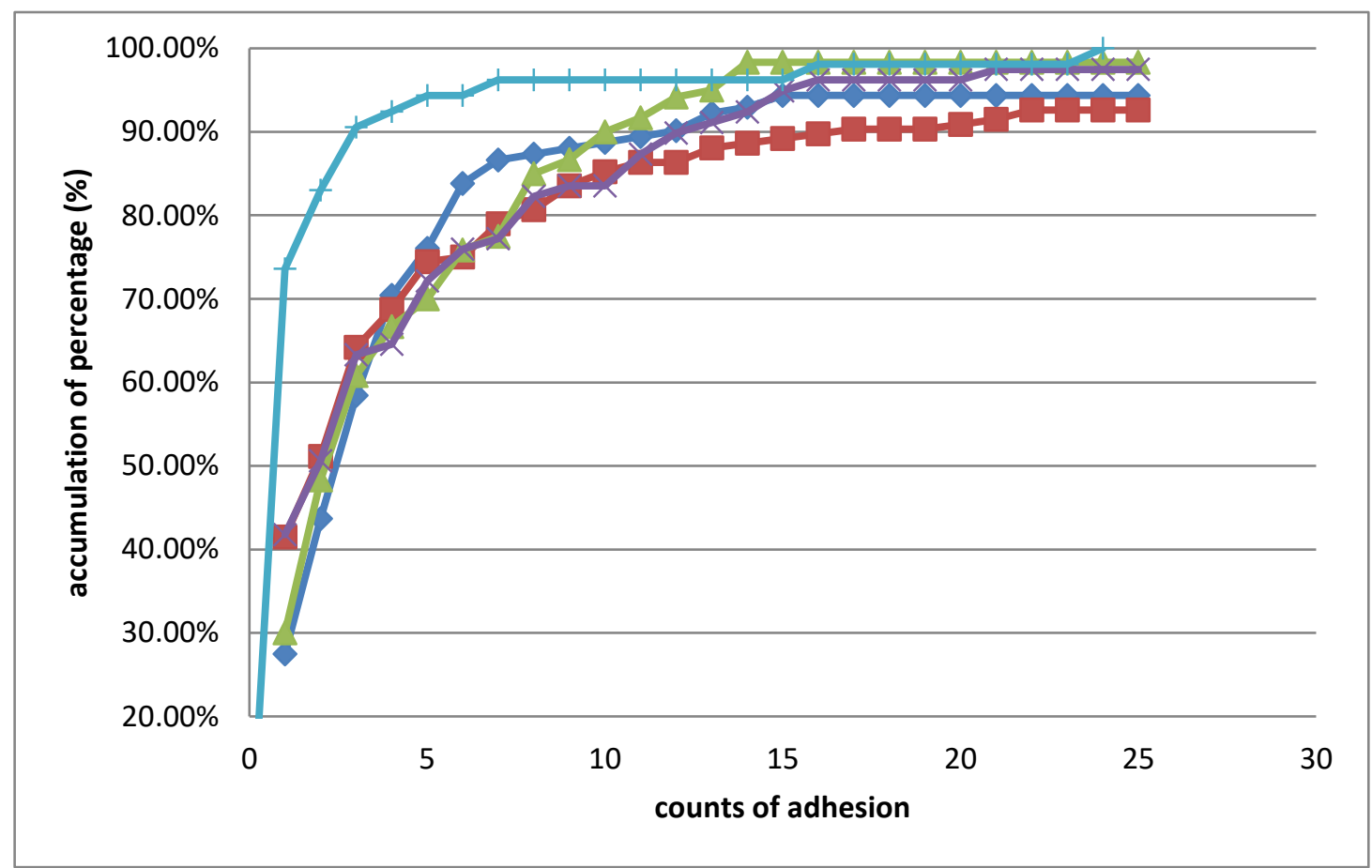

Figure.5. 22. Accumulation of adhesion detected percentage with adhesion type increasing. Blue diamond is for silica colloid probes with NCYC-1324, red square is for silica colloid probes with NCYC-1681, green triangle is for hydroxyapatite colloid probes with NCYC-1324, purple cross is for hydroxyapatite colloid probes with NCYC-1681 and cyan plus is for hydroxyapatite colloid probes with P.F bacteria.

Analysis showed that $90 \%$ of force curves had adhesion events at the surface of both kinds of yeasts. For yeast - silica colloid probe measurement there was a maximum of 16 adhesion events within the adhesion component. When hydroxyapatite colloid probes were applied for the measurements, more than $95 \%$ of the adhesion component in the force curve were found with less than 16 adhesion events, on both NCYC-1324 and NCYC-1681 yeast surfaces. P.F bacteria - 
hydroxyapatite colloid probe contacts contained less adhesion components. About $90 \%$ of the force curves were found to contain 4 or less kinds of adhesion events and $95 \%$ of the force curves had less than 7 adhesion events. The P.F bacteria were not applied for comparison because it lacked adhesion with silica colloid probes as stated in section.5.6.1.

As shown in Figure.5.22, force curves measured with the hydroxyapatite colloid probe had less adhesion events than those measured with silica colloid probes. Associated with the maximum adhesion analysis in section.5.6.1, cell surfaces have more chemical groups that are more adhesive to hydroxyapatite beads than to silica beads. The influences that were induced by adhesion events were concealed in the large adhesion strengths.

Large adhesion event numbers were also found, for example, 55 adhesion events were found in one of the measurements with a silica colloid probe at a NCYC-1324 yeast surface and 38 adhesion events were recorded in one of the force curve measured at a NCYC-1324 yeast with a hydroxyapatite colloid probe. Large adhesion event numbers, as large as 38 (hydroxyapatite - NCYC-1681), 159 (silica NCYC-1681) and 24 (hydroxyapatite - P.F bacteria), were also found in other measurements that were made. The large adhesion event number determined by the code analysis appeared to be induced by an error that occurred by measuring noise. As an example, the maximum number found for NCYC-1681 yeast - hydroxyapatite colloid probe contact (159) was an outlier, the second largest number was 61, this is an unreasonable gap. There was no other measurement recorded with 61 to 159 events. The adhesion components number calculated seemed to be more reliable when the number was less than 5 and the majority of the adhesion event numbers (more than $70 \%$ ) were found to have less than 5 . There were many force curves that were calculated with more than 5 kinds of adhesion events. The criteria of slope analysis for these calculated numbers were manually validated; the curve shapes were significantly different from fluctuations that occurred because of noise. For example, all adhesion events (16) for yeast - silica colloid probe measurements were validated to be induced by adhesion alone and not noise. 


\subsection{The Influence of Cell Influential Molecules on Cell Surface Properties}

An adhesive controlling agent and antibiotics molecules were added in buffers to analyse the influence of these molecules on cell surfaces interactions, electrostatic and adhesion forces. Sodium tripolyphosphate (STP) was used as the adhesive controlling agent and amoxicillin was the antibiotic used in this study. Effects of different concentrations of these compounds were analysed and described in the following subsections.

\subsubsection{The Influence of an Adhesive Controlling Agent on Cell Surface Properties}

(Xiong et al., 2016)

A

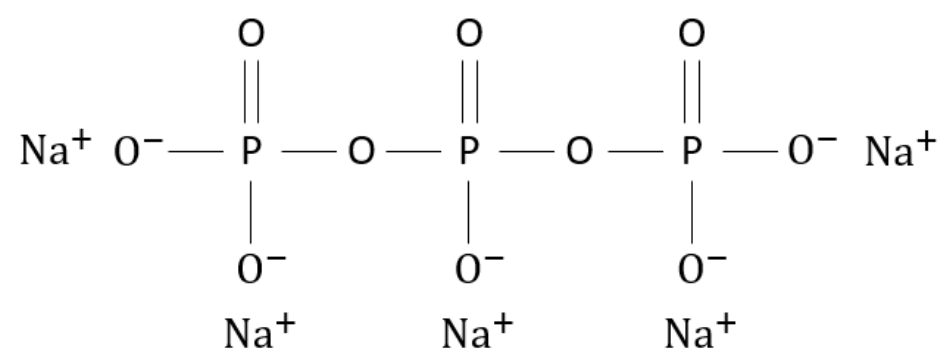

B

protein + triphosphate $\mathrm{t}^{5-} \longrightarrow$ protein - triphosphate $^{4-}$

Figure.5. 23. The chemical structure of STP (A) and reaction mechanism of reaction between cell surface protein and STP (B).STP was added to buffers that immersed the immobilised cell samples in AFM force spectroscopy. Adhesions and zeta potentials were calculated from force curves with the model that was introduced in section.3.2. The influence of adhesive controlling agent on cell surfaces was established by comparison of the adhesion and electrostatics differences that were incident on yeasts and P.F bacteria surfaces in different $\mathrm{pH}$. 


\subsubsection{Influence of Adhesive Controlling Agent on Cell Adhesion}

The 3 kinds of cells were measured in $0.01 \mathrm{M} \mathrm{NaCl}$ and $1 \mathrm{~g} / \mathrm{L}$ STP buffers at different $\mathrm{pH}$. The adhesion forces applied in the interactions were analysed and presented in Table.5.5. Compare to Table.5.4, the addition of STP made no significant impact on the average adhesion. Hydroxyapatite colloid probes had stronger interaction with the yeast cells (section.5.6.1). Silica colloid probes were found to not have enough adhesion on P.F bacteria cell surfaces. Therefore, the adhesion analysis was based on silica-yeast and hydroxyapatite - bacteria interactions.

On comparing Table.5.4 and Table.5.5, more force curves with adhesion were found for the STP study on both kinds of yeasts. The P.F bacteria showed less adhesions at $\mathrm{pH} 5$ and 7 and more adhesion force curves were found at $\mathrm{pH} 9$. The maximum, minimum and average values of adhesion forces between silica colloid probes and yeasts in $\mathrm{NaCl}$ buffer were found to be larger than the adhesion forces measured when the STP molecule was added to the buffers. P.F bacteria adhesion strength was decreased in $\mathrm{pH} 5$ and $\mathrm{pH} 9$, but increased in $\mathrm{pH} 7$ by STP molecules. The adhesion forces measured on the 3 kinds of cells were observed to be mostly in the range between $200 \mathrm{pN}$ to $2000 \mathrm{pN}$. Compared to the adhesion forces measured in buffers without STP, the small adhesion forces (smaller than 100pN) and large adhesion forces (larger than $5 \mathrm{nN}$ ) were less in STP buffers; large adhesion more than $10 \mathrm{nN}$ was not observed.

With the comparison of cells tested in $\mathrm{NaCl}$ buffers (Table.5.4) and STP added buffers (Table.5.5), both yeasts and P.F bacteria were found to have adhesion forces with moderate strengths (200pN to $2000 \mathrm{pN})$. Small and large adhesion forces were all change by addition of the STP molecules.

\begin{tabular}{|r|r|r|r|}
\hline $\mathrm{pH}$ & $\mathrm{pH} 5$ & $\mathrm{pH} 7$ & $\mathrm{pH} 9$ \\
\hline \multicolumn{3}{|c|}{ NCYC-1324 } \\
\hline
\end{tabular}




\begin{tabular}{|c|c|c|c|}
\hline Minimum & 159.6 & 84.1 & 160.8 \\
\hline Maximum & 1847.9 & 2622.6 & 2554.9 \\
\hline Average & 515.7 & 685.4 & 941.9 \\
\hline Count & 31 & 25 & 13 \\
\hline \multicolumn{4}{|c|}{ NCYC-1681 } \\
\hline Minimum & 33.2 & 33.5 & 21.4 \\
\hline Maximum & 2425.1 & 5029.2 & 6663.9 \\
\hline Average & 704.5 & 705.7 & 539.4 \\
\hline Count & 29 & 41 & 54 \\
\hline \multicolumn{4}{|c|}{ P.F bacteria } \\
\hline Minimum & 62.5 & 222.1 & 73.8 \\
\hline Maximum & 97.2 & 5656.0 & 470.3 \\
\hline Average & 85.2 & 1909.3 & 164.7 \\
\hline Count & 3 & 6 & 6 \\
\hline \multicolumn{4}{|c|}{ Force in unit of $\mathrm{pN}$} \\
\hline
\end{tabular}

Table.5. 5. Minimum, maximum, average adhesion force strength and number of force curves detected with adhesion force found in NCYC-1324, NCYC-1681 cerevisiae cell - silica colloid probes interactions and P.F bacteria - hydroxyapatite colloid probes interactions in $1 \mathrm{~g} / \mathrm{L} \mathrm{STP}$ molecule added $0.01 \mathrm{M} \mathrm{NaCl}$ buffers at different $\mathrm{pH}$. 
5.7.1.2. Influence of Adhesive Controlling Agent on Cell Surface Electrostatics
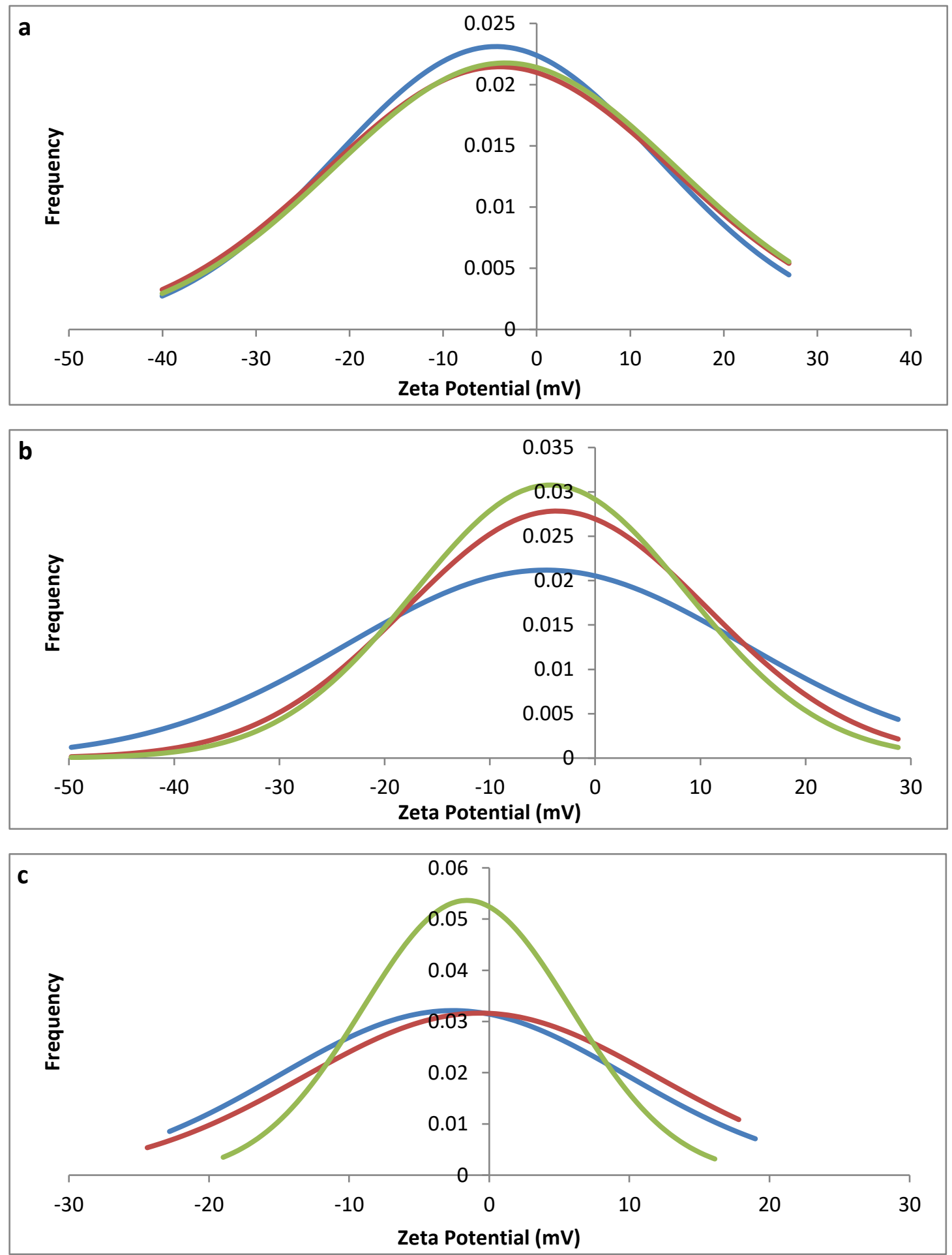

Figure.5. 24. Gaussian distribution estimation for STP added buffer $(0.01 \mathrm{M} \mathrm{NaCl}$ and $1 \mathrm{~g} / \mathrm{L} \mathrm{STP}$ ) measurement, a for NCYC-1324, $\mathrm{b}$ for NCYC-1681 and c for P.F bacteria. $\mathrm{pH} 5$ shows in blue, $\mathrm{pH} 7$ is in red and green for $\mathrm{pH} 9$. Unit of zeta potential is in $\mathrm{mV}$. 
Zeta potentials were calculated with force curves measured on cells in STP added buffer, as shown in 5.23. Average zeta potentials in STP buffer were -3- $-4.5 \mathrm{mV}$ for NCYC-1324 yeasts, $-4--5 \mathrm{mV}$ for NCYC-1681 yeasts and $-0.5--2.5 \mathrm{mV}$ for P.F bacteria. The measurement in buffer without STP showed -9- $-12.5 \mathrm{mV}$ for NCYC-1324 yeasts, $-13--22.5 \mathrm{mV}$ for NCYC-1681 yeasts and $-7--8 \mathrm{mV}$ for P.F bacteria, as described in section.5.3.1. There was a $-6 \mathrm{mV}$ difference between the average zeta potential measured with the Zeta-Sizer and the average zeta potentials calculated from force curves, as analysed in section.5.5. With the consideration of the $-6 \mathrm{mV}$ deviation, the average zeta potentials measured in STP buffers were still less negatively charged. STP molecules made both yeasts and P.F bacteria to be less negatively charged, which may occur due to encouraging the positive charges or restraining the negative charges on cell surfaces.

The standard deviations of zeta potential calculated with force curves measured on cells in STP buffers were found to be around $18 \mathrm{mV}$ for NCYC-1324 yeasts, 13 $19 \mathrm{mV}$ for NCYC-1681 yeasts and $7-12.5 \mathrm{mV}$ for P.F bacteria. Compared with the standard deviation calculated with force curves measured in buffers without STP, described in section.5.5, the distributions of zeta potentials were narrowed in STP buffers, which means the reduction of negative charges would be induced by restraining the negative charges on cell surfaces. Otherwise, more positive charges adsorbed on the cell surfaces would make the cell surface electrostatics more complex and distributed in a wider range of zeta potentials.

${ }^{+}$(Tan et al., 2014)

\subsubsection{Effects of Antibiotics Used on Yeast Cells Adhesion}

(Craig, 1998)

(Zhou et al., 2015)(Selvakumar et al., 2006)0.4mg/L, $1 \mathrm{mg} / \mathrm{L}$ and $4 \mathrm{mg} / \mathrm{L}$ of amoxicillin concentrations were used in the antibiotics influence study. The amoxicillin influence was found to be dependent on time of exposure as well as concentration. Therefore, measurements were made at 15-30 minutes' cells exposure 
time and 60- 75 minutes' cells exposure time in different amoxicillin concentrations. Snap-in forces were observed in and the approach of force curves were found indicating the presence of attractive forces, which were possibly induced by chemical adhesions or van der Waals forces. The attractive forces were found at separation distances in the range from $10-30 \mathrm{~nm}$ between two surfaces. They concealed the zeta potential influence on the force curve, which was only observable in a few nm of distance before contact. Therefore, the presence of attractive forces compromised the determination of zeta potentials from force curves measured at cells surfaces under amoxicillin treatment.

Analysis of amoxicillin influences on NCYC-1324 yeasts showed that amoxicillin concentrations significantly influenced the NCYC-1324 yeast adhesion. For force measurements at NCYC-1324 cells in $0.4 \mathrm{mg} / \mathrm{L}$ amoxicillin buffer most of the cells (more than $80 \%$ ) were with a significant large adhesion (about $10 \mathrm{nN}$ ) after 15-30 minutes exposure time. As shown in Figure.5.25, large adhesions with long adhesion influencing distances and multiple adhesion components were found. There was no significant adhesion found on NCYC-1324 yeasts exposed in amoxicillin for 60- 75 minutes; NCYC-1324 yeasts were also found to be not adhesive when suspended in no amoxicillin buffer for more than one hour. Therefore, $0.4 \mathrm{mg} / \mathrm{L}$ of amoxicillin was increasing the adhesion strength, influencing distance and complexity of the adhesion interaction for NCYC-1324 yeasts. Longer exposure time to $0.4 \mathrm{mg} / \mathrm{L}$ amoxicillin did not induce significant difference from the time of influence on buffers without amoxicillin to untreated systems. 


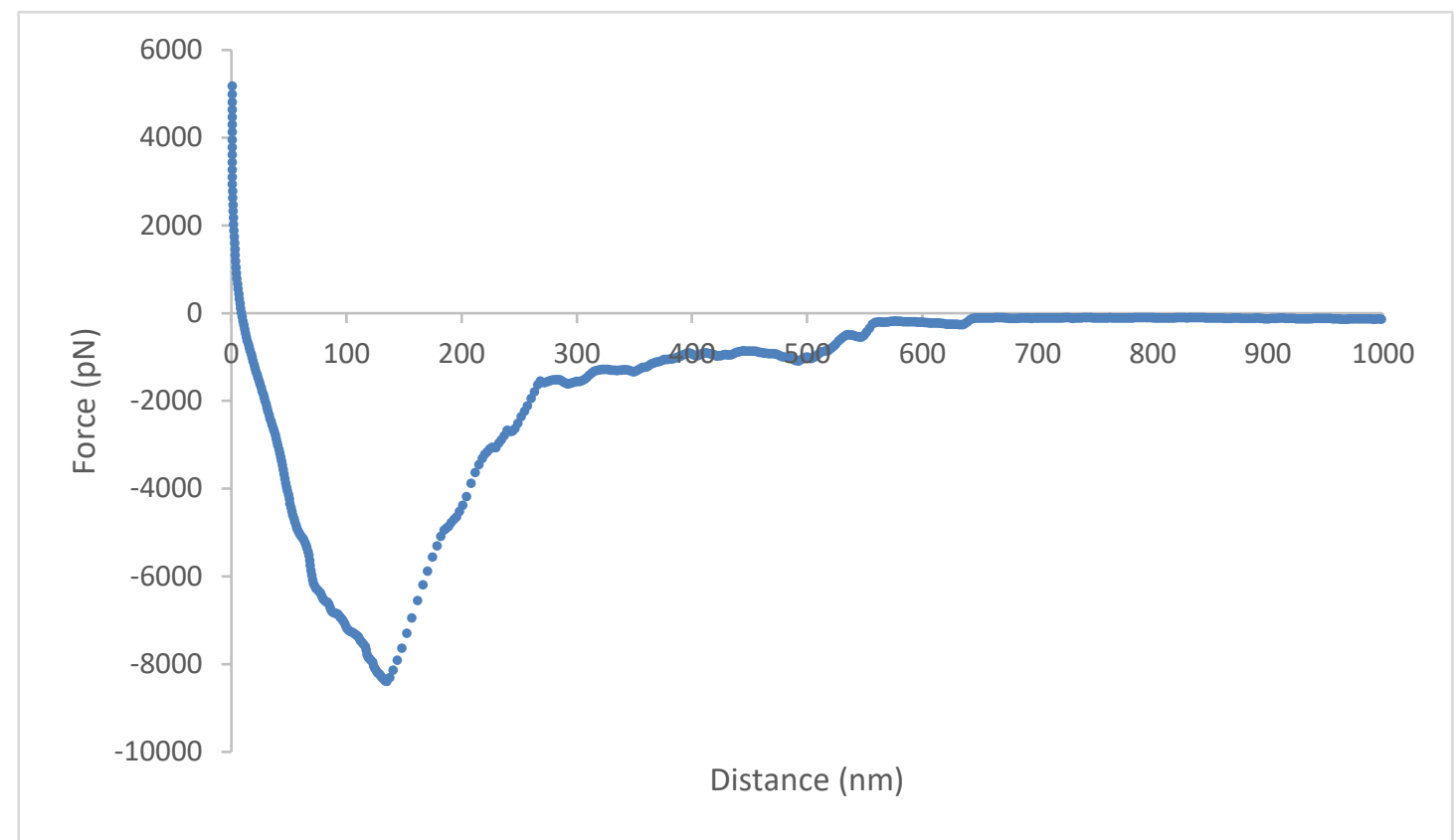

Figure.5. 25. Typical force curve measured on NCYC-1324 yeast cell in $0.4 \mathrm{ml} / \mathrm{L}$ amoxicillin buffer with exposure time of 15 - 30 minutes.

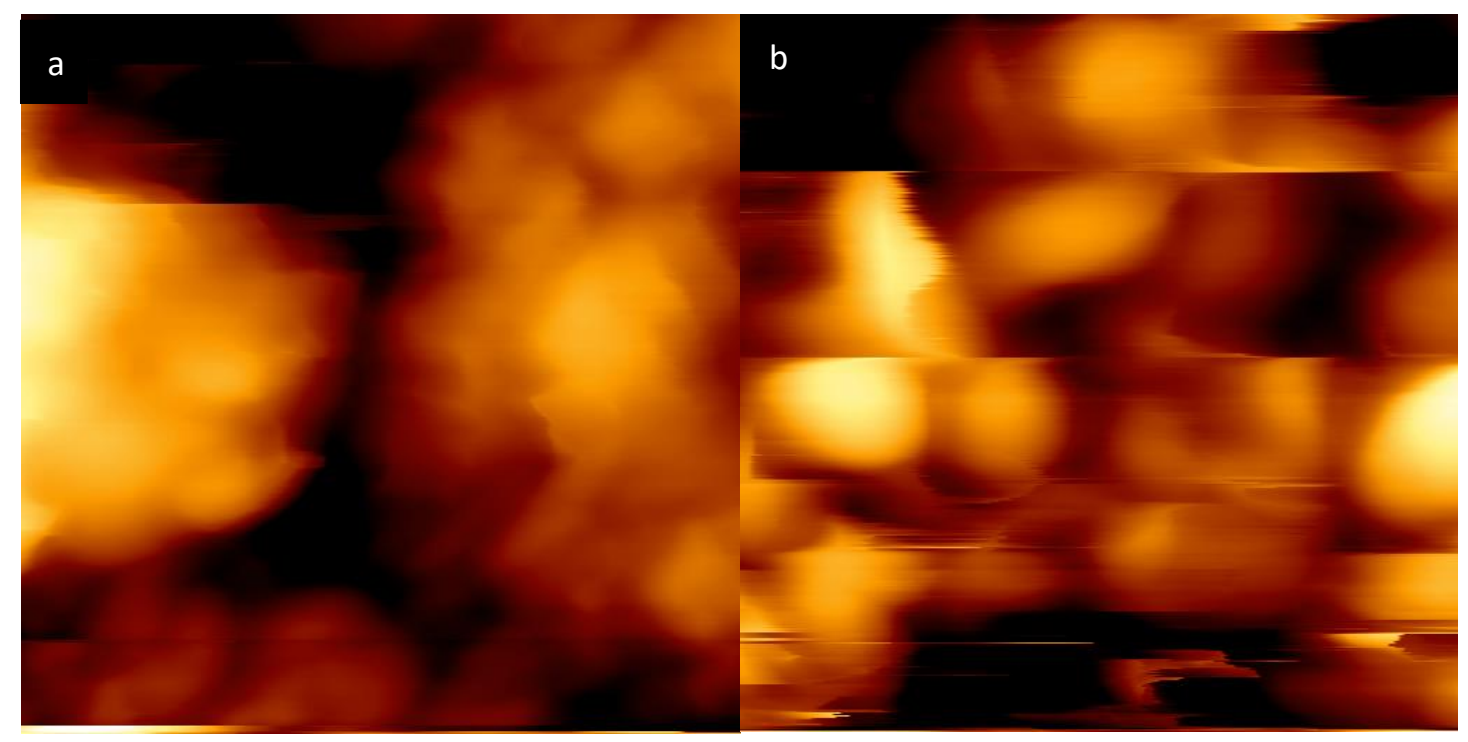

Figure.5. 26. Contact mode imaging of NCYC-1324 yeast cell samples in $0.4 \mathrm{mg} / \mathrm{L}$ amoxicillin buffer (a) exposed for 15 - 30 minutes and (b) $60-75$ minutes. Both images are $50 \mu \mathrm{m}$ square.

Through the contact mode imaging, as shown in Figure.5.25 and 5.26, cells were found to be attached at the surface in all buffers; 15- 30 minutes and 60- 75 minutes suspended in $0.4 \mathrm{mg} / \mathrm{L}$ and $1 \mathrm{mg} / \mathrm{L}$ amoxicillin. From Figure.5.25b, if was observed that the attached cells were shifting on the sample moved on the sample surface by the 
imaging probes, arguably a consequence of the cell membrane attachment forces decreasing with exposure time. Aggregated yeasts were found in both 15-30 minutes and 60- 75 minutes exposure time systems, as shown in Figure.5.25 and 5.26. It was either induced by the more complicated adhesion interactions or weakened cell health. Therefore, the low amoxicillin concentrations $(0.4 \mathrm{mg} / \mathrm{L}$ and $1 \mathrm{mg} / \mathrm{L})$ make the adhesion interactions of the cell more complex and the longer exposure time makes the yeast adhesions negligible.

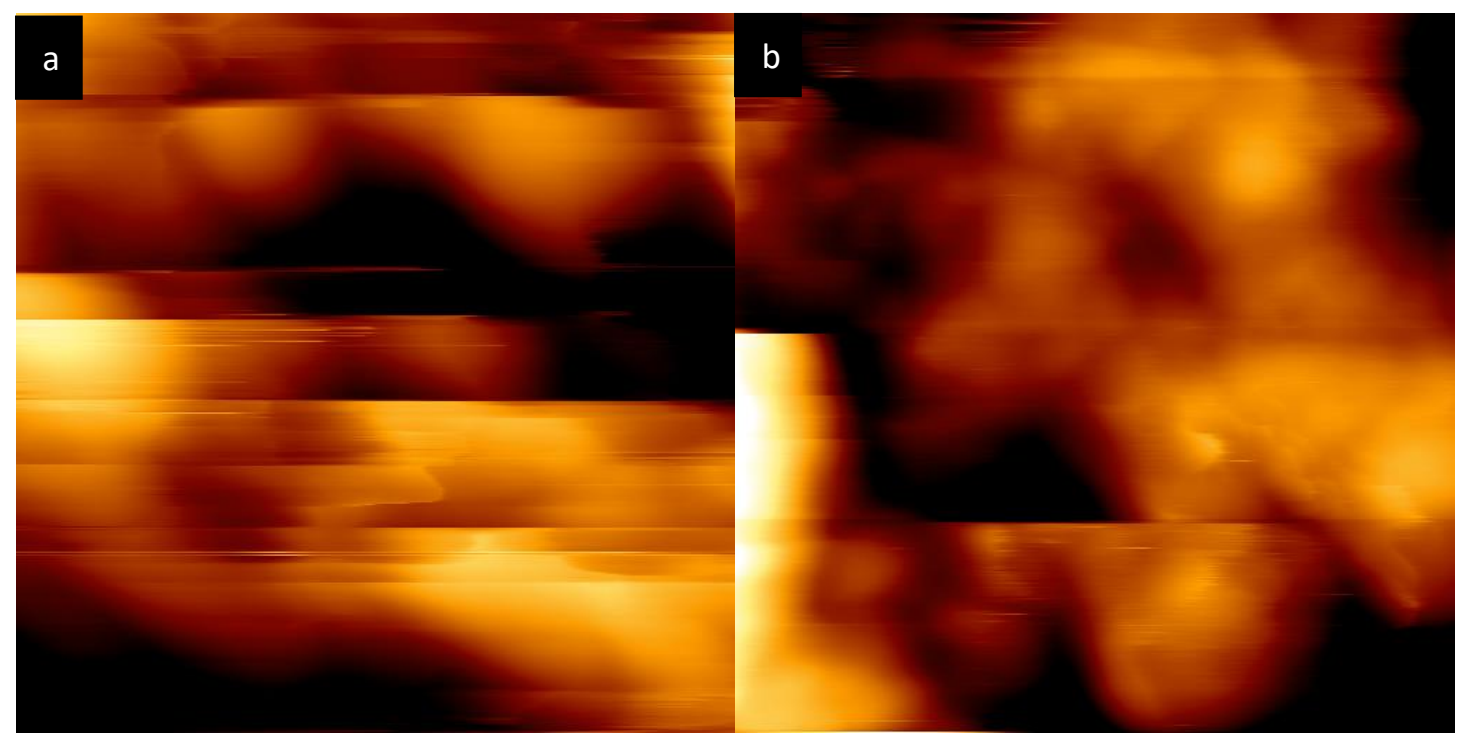

Figure.5. 27. Contact mode imaging of NCYC-1324 yeast cell sample in $1 \mathrm{mg} / \mathrm{L}$ amoxicillin buffer (a) exposed for 15 - 30 minutes and (b) $60-75$ minutes. Both images are $50 \mu \mathrm{m}$ square.

When study was attempted in $4 \mathrm{mg} / \mathrm{L}$ amoxicillin buffers after $15-30$ minutes exposure time it was hard to find an attached cell on the membrane surfaces. The surface was repeatedly scanned but it was a membrane surface without any microbes attached. The sample surfaces did however show that there were soft materials attached, as shown in Figure.5.27, which is of poor quality because of the soft material deforming under action of the probe. Force curves measured at the surface showed that there was no adhesion forces that could be measured for the materials. The imaging and force curves may infer that there might have been very soft cells attached on the surfaces; amoxicillin effects microbial cells by damaging the cell wall structures. The measurements for cell samples after $60-75$ minutes exposure time 
showed no difference for the soft materials (Figure.5.27b) as compared to those measured after 15- 30 minutes exposure time (Figure.5.27a). Thus, it appeared that the amoxicillin influence at $4 \mathrm{mg} / \mathrm{L}$ concentration was rapid and less than 15 minutes. Therefore, 4mg/L was higher than amoxicillin's critical influencing concentration because the influence of amoxicillin was significant.

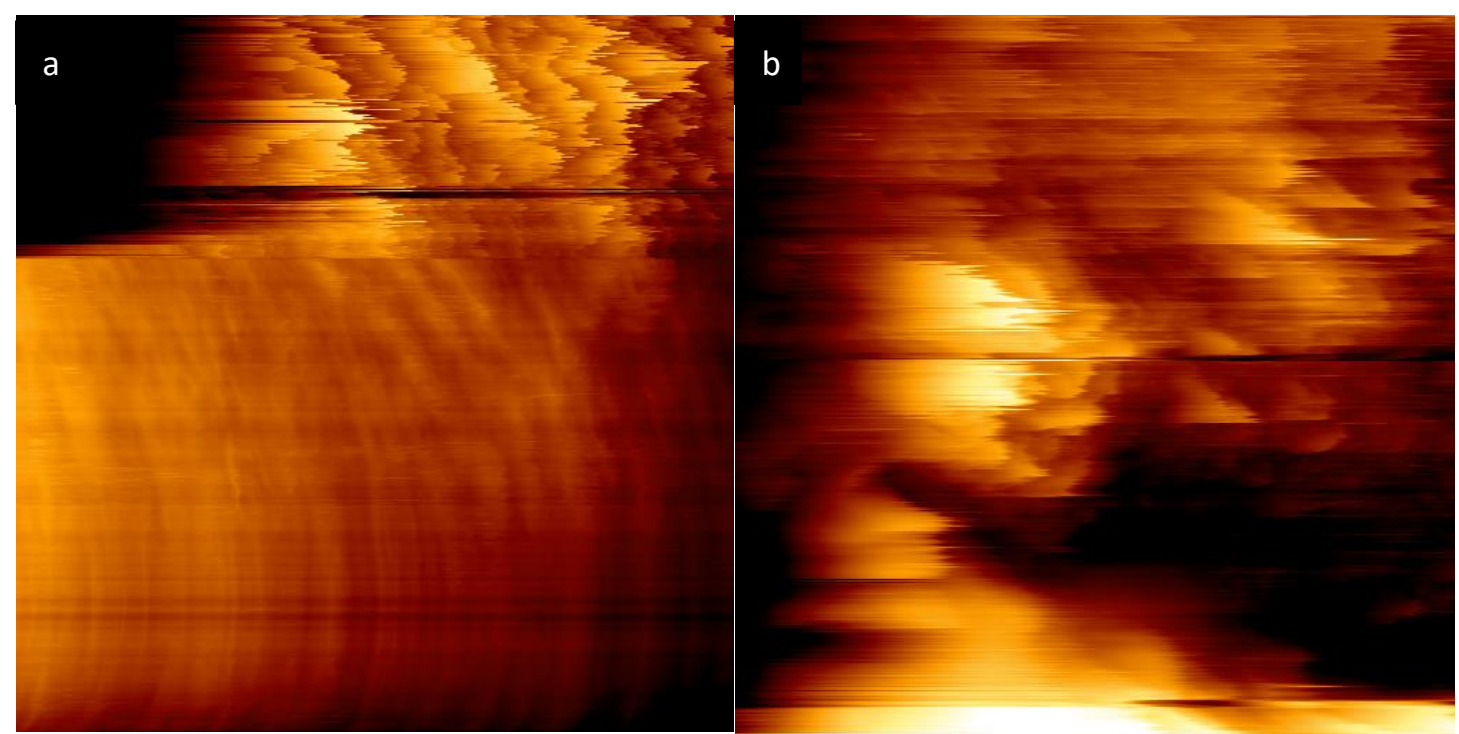

Figure.5. 28. Contact mode imaging of NCYC-1324 yeast cell samples in $4 \mathrm{mg} / \mathrm{L}$ amoxicillin buffer (a) exposed for 15 - 30 minutes and (b) $60-75$ minutes. Both images are $50 \mu \mathrm{m}$ square.

Concentrations of $0.4,1$ and $4 \mathrm{mg} / \mathrm{L}$ amoxicillin were applied for study of NCYC-1681 yeasts as well. Measurements at NCYC-1681 yeast surfaces showed more significant difference to those measured at untreated yeast surfaces, when compared with NCYC-1324 yeast cells. After $15-30$ minutes exposure to $0.4 \mathrm{mg} / \mathrm{L}$ of amoxicillin, $50 \%$ of the NCYC-1681 cells had an adhesive component in the force curve measured at their surface. The adhesion strength was about $2 \mathrm{nN}$, significantly smaller than that found on NCYC-1324 yeasts in the same condition (Figure.5.25). No adhesive yeast was found when exposure time was $60-75$ minutes inferring the adhesion was weakened with the significant increase in the exposure time.

In the two exposure times used, for the majority of the imaging no single NCYC-1681 yeast cells were observed. However, aggregations of NCYC-1681 yeasts were found on the membrane surfaces, as shown in Figure.5.28. Therefore, the 
$0.4 \mathrm{mg} / \mathrm{L}$ amoxicillin concentration made a significant influence on NCYC-1681 yeasts, but it was not fast as cell aggregations were still found on the samples after 60-75 minutes exposure time.

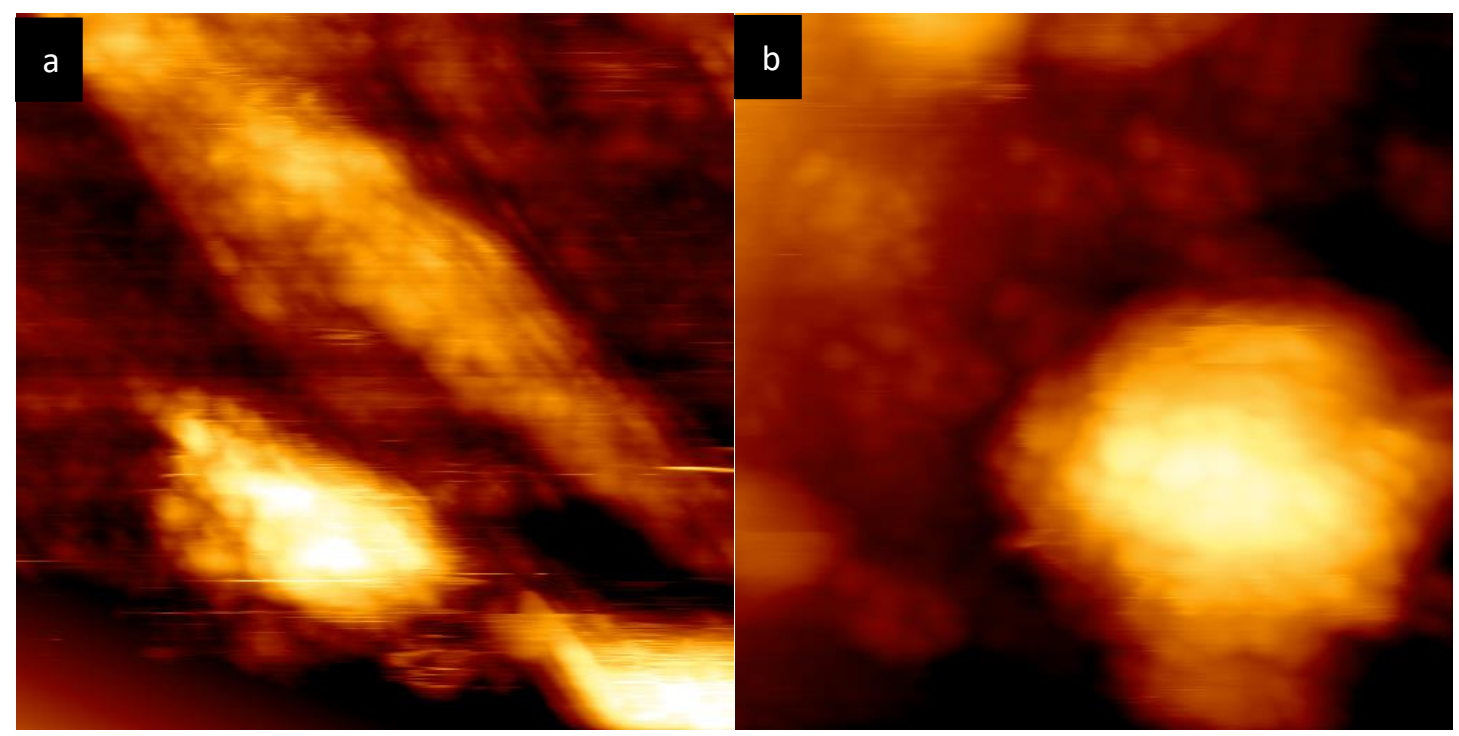

Figure.5. 29. Contact mode imaging of NCYC-1681 yeast cell sample in $0.4 \mathrm{mg} / \mathrm{L}$ amoxicillin buffer (a) exposed for 15 - 30 minutes and (b) 60 - 75 minutes. Both images are $50 \mu \mathrm{m}$ square.

For the measurement on NCYC-1681 yeasts after 15-30 minutes exposure time, largest adhesions were found to be $3.5 \mathrm{nN}$ in $1 \mathrm{mg} / \mathrm{L}$ amoxicillin buffers and most force curves showed no adhesion forces. When the amoxicillin concentration was as large as $4 \mathrm{mg} / \mathrm{L}$, no adhesion was found. The cell surfaces were seriously influenced by treatment with $1 \mathrm{mg} / \mathrm{L}$ and $4 \mathrm{mg} / \mathrm{L}$ amoxicillin, as suggested by the fact that the imaging of NCYC-1681 yeasts in the two amoxicillin concentrations was difficult and full images were not achieved. Force curves were measured on the scanned cells before the imaging collapsed. The measurements after longer exposure time (60-75 minutes) showed that there was no difference with the application of more immersing time. Thus, the critical influencing concentration of amoxicillin on NCYC-1681 yeasts was found to be between $0.4 \mathrm{mg} / \mathrm{L}$ and $1 \mathrm{mg} / \mathrm{L}$. 


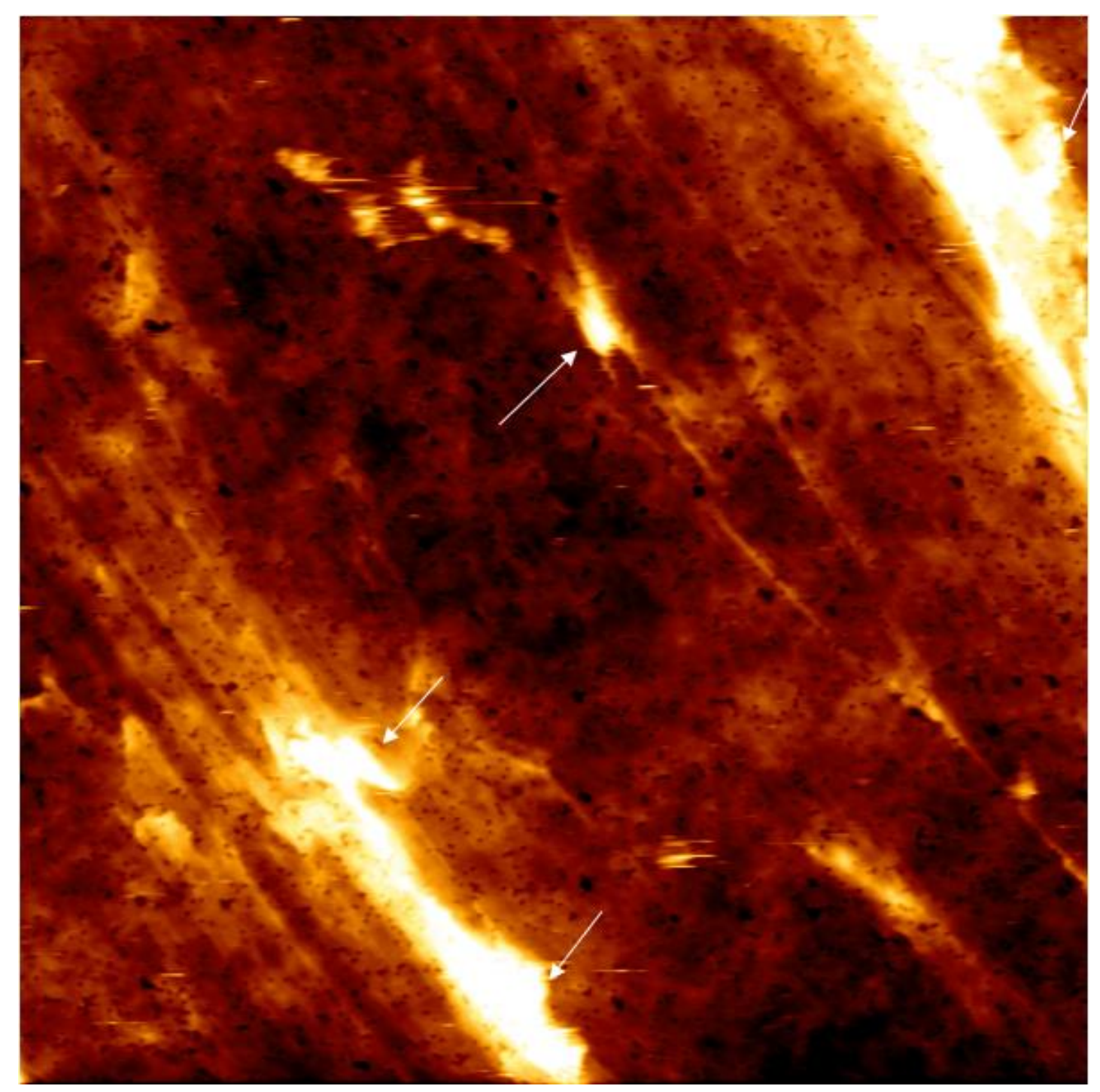

Figure.5. 30. Typical AFM contact mode imaging of P.F bacteria sample surfaces measured in amoxicillin buffers, imaging in scale of $50 \mu \mathrm{m}$.

The imaging of P.F bacteria in amoxicillin buffers was found to be not significantly influenced by different amoxicillin concentrations $(0.4 \mathrm{mg} / \mathrm{L}, 1 \mathrm{mg} / \mathrm{L}$ and $4 \mathrm{mg} / \mathrm{L})$ and exposure times ( $15-30$ minutes and $60-75$ minutes $)$, an example is shown in Figure.5.29. Thus, amoxicillin influences were not able to be identified from imaging comparisons. However, single PF cells could be observed and therefore, the force curves measured were applied on single cells accurately.

The adhesion forces were measured on the P.F bacteria in different amoxicillin concentrations and exposure times. About $50 \%$ of the force curves were found with adhesion forces and the majority of the adhesion forces were smaller than $500 \mathrm{pN}$. The 
average values were not significantly influenced by different amoxicillin concentrations and exposure times, and ranged from $100-300 \mathrm{pN}$ for all measuring conditions.

The adhesion forces were analysed as a data set and presented as Gaussian distributions as shown in Figure.5.30 to estimate the maximum range of adhesion that could occur (with standard deviation). In low amoxicillin buffers $(0.4 \mathrm{mg} / \mathrm{L}$ and $1 \mathrm{mg} / \mathrm{L})$, the short exposure times (15-30 minutes) maximum adhesion forces were estimated to be about $200 \mathrm{pN}$ and maximum increased to $800 \mathrm{pN}$ in $0.4 \mathrm{mg} / \mathrm{L}$ amoxicillin buffers and 500pN in $1 \mathrm{mg} / \mathrm{L}$ amoxicillin buffers after long exposure times (60-75 minutes), as shown in Figure.5.30a and b. Theoretically, $1 \mathrm{mg} / \mathrm{L}$ amoxicillin buffers and longer exposure time would provide stronger amoxicillin influence on the P.F bacteria. In high amoxicillin concentration $(4 \mathrm{mg} / \mathrm{L})$, the maximum estimate of adhesion force was found to be more than $800 \mathrm{pN}$ after short exposure times (15-30 minutes) and decreased to $200 \mathrm{pN}$ after long exposure times (60-75 minutes). Thus, the cell surface adhesion forces increased and then decreased with the amoxicillin influence becoming stronger on P.F bacteria and the amoxicillin influence on P.F bacteria required a long exposure time to complete its effect process.

From the study of amoxicillin effects on P.F cell studied by Zhou et al. in 2015 (Zhou et al., 2015), P.F bacteria showed a relatively high resistance to amoxicillin. Compared to P.F bacteria, yeasts were influenced rapidly in $4 \mathrm{mg} / \mathrm{L}$ amoxicillin buffers. While, the critical concentration for amoxicillin treating P.F bacteria was larger than $4 \mathrm{mg} / \mathrm{L}$, as cells survived of $4 \mathrm{mg} / \mathrm{L}$ amoxicillin buffers lasted for longer than the test period (60-75 minutes). 

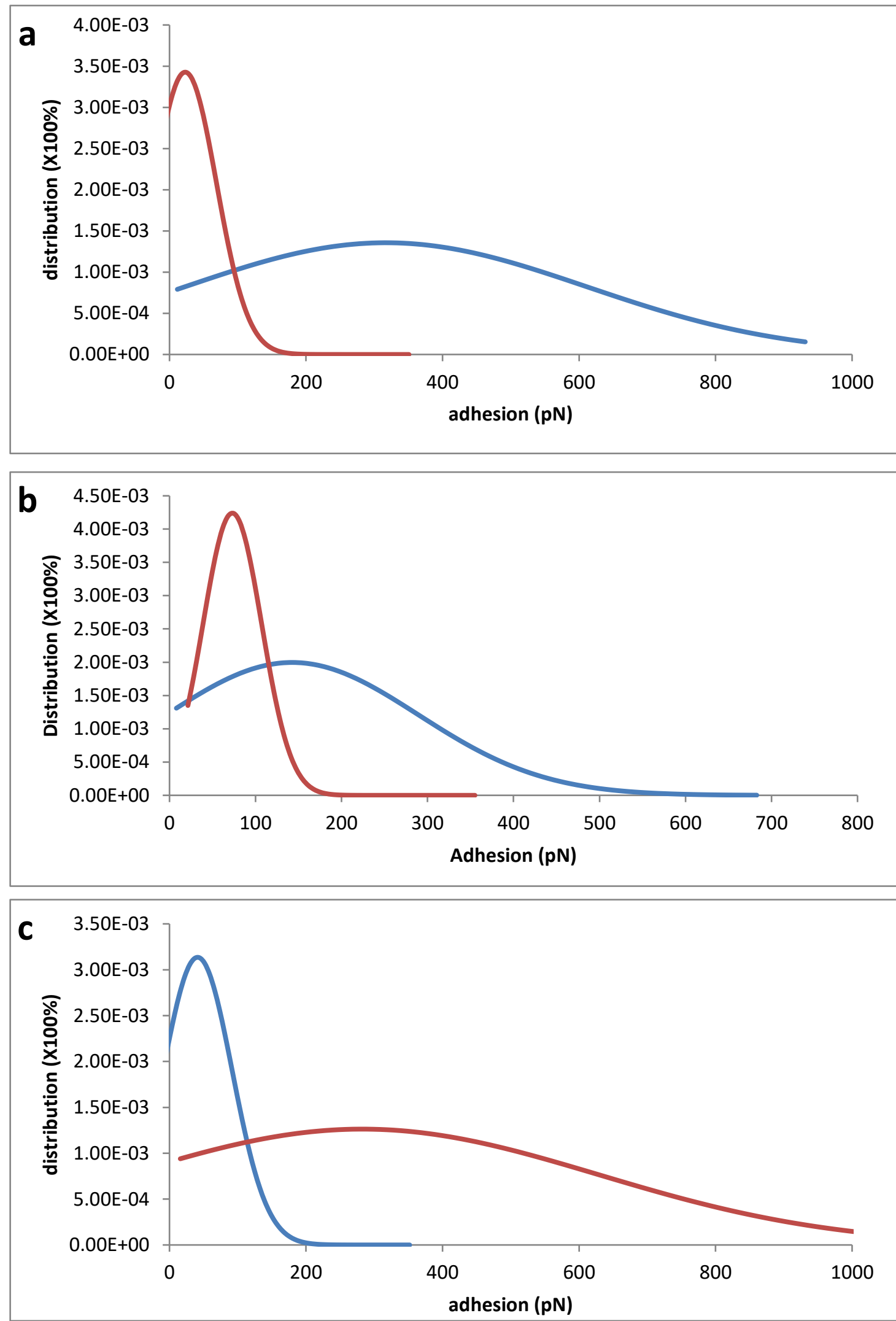

Figure.5. 31. The Gaussian distribution analysis plot of amoxicillin of $0.4 \mathrm{mg} / \mathrm{L}(\mathrm{a})$, $1 \mathrm{mg} / \mathrm{L}(\mathrm{b})$ and $4 \mathrm{mg} / \mathrm{L}(\mathrm{c})$ with the difference of exposure time 15 - 30 minutes (red) and $60-75$ minutes (blue). 
The influence of amoxicillin was also more significant on yeasts than that found on P.F bacteria. Therefore, in comparison to the 3 kinds of cells, the influence of amoxicillin was found as strongest on NCYC-1681, then NCYC-1324 and weakest on P.F bacteria.

\subsection{Conclusion}

In this chapter, force curves were measured on yeasts (NCYC-1324 and NCYC-1681) and bacteria (Pseudomonas fluorescens), in conjunction to calculation model to determine zeta potential, adhesion and their distributions. Hydrophobicity of cell surfaces were measured and used to analyse the buffer influences on cell surface chemistry, which was related to the surface electrostatic interactions.

Force curves were used to calculate zeta potentials on cells, which were found to deviate from the Zeta-Sizer measurements. Based on the Gaussian distribution and estimated zeta potentials at individual cells, the zeta potentials of cells were estimated as average values with standard deviations in different buffer conditions. Compared to measuring with the Zeta-Sizer, differences from 5 to $6 \mathrm{mV}$ were found between force curves calculated zeta potentials and Zeta-Sizer measurements in different buffer conditions. The combination of both methods was applied and found to provide a more comprehensive analysis of cell surface electrostatics, which optimised the average zeta potentials applied in the distribution.

The cell hydrophobicity estimation from solvent partitioning techniques were used to analyse the cell surface chemistry with calculations that were developed from Gaussian distribution and reaction equilibrium. The surface chemistry distribution on cell surfaces in different buffer conditions were analysed with the model. Analysis showed there was a correlation relationship between zeta potential determined by AFM and the hydrophobicity, for all cell types.

Zeta potential analysis on cell surfaces was studied at different buffer $\mathrm{pH}$, ionic strengths and in the pressure of additional molecules' (STP and amoxicillin). The zeta 
potentials were found to be significantly affected by $\mathrm{pH}$, ionic strengths and STP molecules. Higher $\mathrm{pH}$ made cells more negatively charged and higher ionic strength made cells less negatively charged. With the calculations based on DLVO theories and different adsorption isotherms, buffer ionic strengths effects were related more to a reversible surface reaction on the cell surface chemistry and buffer ions.

The effects of STP molecules made the cell surfaces become less negatively charged; this was caused by the interference of the structure of surface charged chemicals. STP molecules provided a significant effect on the distribution range of zeta potentials. The standard deviations of distribution were found to be smaller with STP added in buffers. The zeta potentials were not able to be calculated from force curves measured on cells in the amoxicillin buffers. Amoxicillin seriously influenced the cell surface chemistry. It made the attractive forces become significant in most approach sections of force curves, which acted to conceal the zeta potential by the convolution of forces.

Adhesion analysis of cell surfaces were applied on the 3 kinds of cells, adhesion strengths and different adhesion components applied were both used in this study. Comparisons of adhesion forces were measured on cells suspended in amoxicillin and STP buffers. The amoxicillin influence was time dependent, which in the measurements made in buffers under its estimated critical influencing concentration and longer exposure time had strong influence on cells. Amoxicillin influences were found to be the strongest on NCYC-1681 yeasts with a critical influencing concentration between 0.4 and $1 \mathrm{mg} / \mathrm{L}$, then moderate on NCYC-1324 yeasts with a critical influencing concentration between $1 \mathrm{mg} / \mathrm{L}$ and $4 \mathrm{mg} / \mathrm{L}$ and weakest on P.F bacteria with a critical influencing concentration of more than $4 \mathrm{mg} / \mathrm{L}$. 


\section{Chapter VI Conclusion}

\subsection{Research Achievements}

The research detailed in this thesis has extended the force analysis of atomic force microscopy (AFM) by closer examination of force-distance curves measured between a colloid probe and the surfaces of filtration membranes and microbial cells. This was the main aim of the thesis and was achieved by the application of a theoretical framework based on DLVO theories and the Possion-Boltzmann Equation (PBE) used in the novel analysis of colloidal interactions and AFM. This aim, and the direction of research, was inspired by a gap analysis of the research literature which examined work such as that by Ruiz-Cabello et al. who demonstrated the possibility of the direct calculation of the electrostatic interactions between two colloidal particles (Ruiz-Cabello et al., 2014) and Brant et al. who simulated the intermolecular and electrostatic interactions based on DLVO theories and PBE (Brant et al., 2006). Further research direction for interpretation of AFM force-distance data was inspired by previous research such as that by McNamee et al. who found that electrostatic forces were related to surface chemical bonding and hydrophobicity forces (McNamee et al., 2006) The first research objective achieved was the establishment and validation of a model for zeta potential calculation from AFM force curves measured at microbial cell and membrane surfaces. Validation was achieved by examining the sensitivity of the model and by comparing calculated values with previous research and measurements using established techniques (streaming potential and Zeta-Sizer). Zeta potential was analysed with the influence of different buffer $\mathrm{pH}$, ionic strength and the environmental additives amoxicillin and STP). Thus, achieving another objective of the study to use the model to extend characterisation of surfaces by AFM, by measuring zeta potential across surfaces. This was further extended by employing methods based on AFM mapping mode, to examine the heterogeneity of zeta potential across filtration membrane surfaces. Adhesion was also calculated from the AFM retraction force curves, and adhesion components were studied for more 
comprehensive adhesion force analysis. A novel approach based on gradient calculation was applied to identify the components in adhesion sections of the AFM force curves. Further characterisation of surfaces was achieved when the third objective of the study was met with the development of a novel calculation model to analysis solvent partitioning data to reveal chemical bonding information in hydrophobicity analysis of microbial cells. Thus, the study provided further novel analysis of the membrane and microbial cells systems by examining the relationship of hydrophobicity, electrostatics and chemical bonding

The study of zeta potentials at membrane surfaces used from different force curves, and so these were analysed with statistical analysis for distribution information. Membrane and microbial cell surfaces were measured in different buffer ionic strengths and $\mathrm{pH}$. Mann-Whitney $\mathrm{U}$ test (MWW) showed that buffer changes will significantly change the zeta potential distribution on both filtration membranes and cell surfaces. On the membrane surfaces, zeta potentials were calculated as an average with a standard deviation and compared with previous zeta potential studies; the average zeta potentials measured from streaming potential were close to the estimation from the AFM method and within its standard deviation. Therefore, the distribution of the AFM data was regarded as the zeta potential heterogeneity across the filtration membrane surface. Zeta potential mapping was also applied, a further development of this study, and displayed the zeta potential heterogeneity on filitration membrane surfaces. Study on buffer ionic strength and $\mathrm{pH}$ influence showed that both conditions are impactive on membrane surface zeta potential distribution.

Adhesion on filtration membrane surface was also analysed. In the present study, adhesion was found to be randomly influenced by the buffer ionic strength and $\mathrm{pH}$. Simple types of adhesion were found and both large and small adhesion strengths were detected. In the membrane systems studied, membrane surface adhesion was found not to be governed through buffers but depended more on membrane surface chemical properties.

Zeta potential on cell surfaces was also analysed as well. Zeta potential distribution was calculated with statistical analysis. Comparison of the average cell 
zeta potential estimated with AFM force curves calculation, with Zeta-Sizer measurements showed that there is a difference of less than $\pm 6 \mathrm{mV}$ between this mature technology and AFM method. While, the standard deviation is normally more than $\pm 10 \mathrm{mV}$. The calculation basis of the AFM method is only a few tens of force curves, with the consideration of low sample basis and standard deviations, the calculated results were to be with reliable standard deviations and average values within reasonable error ranges. Thus, to optimize the method, a larger sample amount could provide more accurate zeta potential estimation.

Influences of buffer ionic strength and $\mathrm{pH}$ were analysed for yeast and bacteria as well with the bioactive molecules STP and amoxicillin added to buffers. These were all found to influence on cell surface zeta potential. Amoxicillin induced the collapsing of cells and this could be detected by the AFM method; the collapsing was significant enough to be observed with cell surface imaging.

The adhesion on microbial cell surfaces was detected as well. With the 3 kinds of cells used, both yeasts showed a good adhesion on silica and hydroxyapatite colloid probes. However, for P.F bacteria, there was little adhesion to both kinds of colloid probes. While, hydroxyapatite colloid probes measured largest adhesions for all 3 kinds of cells. The adhesion components were found to be complex. More than 16 kinds of adhesion components could be found in one force curve, $80 \%$ of the force curves were found to be with less than 7 kinds of adhesion components for yeasts and P.F bacteria. STP was found to stimulate moderate strength adhesions $(200-2000 \mathrm{pN})$, and inhibit the large or small adhesions on yeasts and bacteria. Comparison of amoxicillin influences on different cells adhesions showed that the impact of amoxicillin was significantly slower on P.F bacteria compared to yeast cells. Adhesions on both yeast cell surfaces was significantly removed by amoxicillin, while, the impact on P.F bacteria adhesion was not as significant as yeast cells. P.F adhesion distribution was found to be enlarged in low amoxicillin concentration and reduced in high amoxicillin concentration with longer exposure time.

Hydrophobicity of cells were measured as solvent partitioning based on optical density (OD) test. Compared previous research using this novel method, a new 
calculation model was built to analyse the OD measured instead of directly using measured results. Reaction equilibrium and Gaussian distribution were applied in the model development to reveal the cell surface hydrophilic chemical properties from the measured OD. The hydrophobicity was transformed to chemical information on cell surfaces in the form of percentage and Gaussian distribution parameters. With the analysis of the developed model, ionic strength was found to play different roles in influencing the surface hydrophilic chemical reaction saturation on yeasts and bacteria cell surfaces. While, there will be more hydrophilic chemical groups on the yeast cell surface increased with increasing $\mathrm{pH}$ and reduced with increasing $\mathrm{pH}$ for P.F bacteria. Zeta potential on all cell surfaces were found to be increasing with increasing $\mathrm{pH}$. In the present study, considering the relationship of hydrophobicity, chemical properties and electrostatics, the hydrophilic chemicals of the yeast cell surfaces increase the negative charge on the cell surfaces, while the same kinds of chemical groups are providing an opposite influence on P.F bacteria surface electrostatics.

\subsection{Application of Research Achievements and Future Research}

This study is a multidisciplinary research program based on colloidal science to characterise on membrane technology and microbial systems. There are many applications for the developed research that can be applied on support further research. Zeta potential characterisation, as a major focus of the thesis aim, and subsequent control shows potential application in governing membrane filtrations and microbial cell behaviour. With the mathematical and scientific theories applied in the computational model of the present study, there are more parameters that can be calculated, such as Young's modulus, and adhesion energy. The application of AFM provides the possibility of analysing the heterogeneity of cells and membrane surfaces. Statistical analysis has been applied in this study and determine the distribution of zeta potential on both microbial cells and filtration membranes. Assisted with more mature measuring methods and with more force curves, the AFM method can provide 
an accurate estimation of surface zeta potential and distribution. While, other measuring methods, such as streaming potential, are hard to provide distribution of samples.

With the capability of zeta potential mapping by AFM, this could provide a new characterisation research direction of how zeta potential heterogeneity influences the membrane filtration. Normally, research focuses on the influence of average zeta potential change, measured with streaming potential. For example, in the streaming potential measured by Oatley-Radcliffe et al., Figure.4.7, there are difference of zeta potential measured in the same buffer condition (Oatley-Radcliffe et al., 2017). Possibly, the difference measured may be explained with zeta potential heterogeneity analysis. As in this study, distribution may be different even in the same average zeta potential. The difference of zeta potential with location change on membrane surface also shows the possibility of mapping membrane electrostatics properties. Kelvin probe force microscopy (KPFM) can measure the surface potential distribution of a flat surface as well. However, KPFM cannot be used in ionic activated solutions (Collins et al., 2015). Thus, to measure the zeta potential in an aqueous environment, the application of the AFM colloid probe method and the calculation model becomes important in study.

In cell study, medical researchers are focused on drug delivery with colloids. Some studies have also used AFM to do the interaction measurement (Han et al., 2005; Lee et al., 1994; Pyo et al., 2006). In this study, a system of analysis of cell surface properties using AFM has been developed and applied to the surface characterisation of NCYC-1324, NCYC-1681 S. cerevisiae and P.F bacteria in different aqueous environments.

With the developed model, adhesion can be analysed more comprehensively. Zeta potential can also be calculated, which has not been achieved with AFM by previous cellular studies. With the concept of the relationship between electrostatics, hydrophobicity and chemical properties, zeta potential can provide estimation of cell surface properties which has not been achieved before. The hydrophobicity analysis model of the thesis can give relationship between hydrophobicity and chemical 
properties as well. With the cooperation of the two models of the thesis, these phenomena on cell surfaces can be studied together. The influence of chemical properties on zeta potential was compared in this study. With the consideration of model development on hydrophobicity analysis, it is possible that the relationship between chemical properties and zeta potential can be designed into a calculation model in the future. Hydrophobicity analysis shows that the surface chemicals can be expressed in the form of a percentage of a distribution value. With the concept of cooperation of AFM and other characterisation methods, such as Raman spectroscopy and nuclear magnetic resonance (NMR), it is possible to provide a more comprehensive estimation of surface chemical analysis than only the measurement of an average value.

Therefore, the developed experimental and analytical methods of the thesis could be applied in drug delivery study for more kinds of cell demonstrated by the work on amoxicillin. Potentially, more kinds of drugs can be tested using this method. As the zeta potential and adhesion are both related to cell surface chemicals, it is possible that these will be influenced by the action of the drug molecules on the cell surface chemical structures, such as glycans and proteins. The AFM measurement and analysis can identify the level of drug's effects on these chemistries without the interruption of other surface chemical structures, which is hard to achieve with Raman spectroscopy or NMR.

The measurement of the thesis demonstrated the possibility of analysing particle-particle interactions; contact of one colloid particle and a single cell was achieved. More properties could be monitored with further development of the calculation model. For example, in biological simulation studies, a single cell under a load has been simulated with a discrete element method (DEM) (Gardiner et al., 2015). The model of the present study could be extended to examine using AFM the mechanical and interaction relationships important to this study. Similarly, the AFM method could benefit research on systems such as particles moving under a load ((Lobo-Guerrero \& Vallejo, 2006) and vesicles moving in a bed (Gera et al., 1998). Applied with the AFM study and calculation, these simulations could reveal more 
information, such as drug delivery and aggregation, instead of only the mechanism of contact and movement trajectory of colloid or cells.

The zeta potential of particles is an important determinant of their behaviour within a colloidal system and the stability of the suspension. Thus, the research of the thesis has the potential to impact the colloid research and industry communities. For example, PM2.5 control studies, simulations based on extension of the present study could identify the most effective method to reduce PM2.5 particles number. Thus, the research achievements of the present study can directly benefit and guide the potential of future research goals in not only the study of membrane filtration and microbial cell control within medicine, but in other fields, such as process and pharmaceutical industries and the environment. 


\section{Reference}

Afonso, M. D., Hagmeyer, G., \& Gimbel, R. (2001). Streaming potential measurements to assess the variation of nanofiltration membranes surface charge with the concentration of salt solutions, 23, 529-541.

Aggarwal, S., Poppele, E. H., \& Hozalski, R. M. (2010). Development and testing of a novel microcantilever technique for measuring the cohesive strength of intact biofilms. Biotechnology and Bioengineering, 105(5), 924-934.

Al-Amoudi, A., Williams, P., Mandale, S., \& Lovitt, R. W. (2007). Cleaning results of new and fouled nanofiltration membrane characterized by zeta potential and permeability. Separation and Purification Technology, 54(2), 234-240.

Alsteens, D., Dague, E., Rouxhet, P. G., Baulard, A. R., \& Dufre, Y. F. (2007). Direct Measurement of Hydrophobic Forces on Cell Surfaces Using AFM, (8), 1197711979.

Barthel, E. (2008). Adhesive elastic contacts: JKR and more. Journal of Physics D: Applied Physics, 41(16), 163001.

Beers, R. F., \& Sizer, I. W. (1951). A Spectrophotometeric Method for Measuring the Breakdown of Hydrogen Peroside by Catalase. The Journal of Biological Chemistry.

Bellona, C., \& Drewes, J. E. (2005). The role of membrane surface charge and solute physico-chemical properties in the rejection of organic acids by NF membranes. Journal of Membrane Science, 249(1-2), 227-234.

Bowen, W., Hilal, N., Lovitt, R., \& Wright, C. (1998). Direct Measurement of Interactions between Adsorbed Protein Layers Using an Atomic Force Microscope. Journal of Colloid and Interface Science, 197(2), 348-52. Retrieved from http://www.ncbi.nlm.nih.gov/pubmed/9466876

Bowen, W. R., Doneva, T. a., \& Stoton, J. A. G. (2002). The use of atomic force microscopy to quantify membrane surface electrical properties. Colloids and Surfaces A: Physicochemical and Engineering Aspects, 201(1-3), 73-83.

Bowen, W. R., Hilal, N., Jain, M., Lovitt, R. W., Sharif, A. O., \& Wright, C. J. (1998). 
The effects of electrostatic interactions on the rejection of colloids by membrane pores-visualisation and quantification. Chemical Engineering Science, 54(3), $369-375$.

Braga, P. C., \& Ricci, D. (2004). Atomic Force Microscopy: Biological Methods and Applications. New Jersey: Humana Press.

Brant, J. a., \& Childress, A. E. (2002). Membrane-Colloid Interactions: Comparison of Extended DLVO Predictions with AFM Force Measurements. Environmental Engineering Science, 19(6), 413-427.

Brant, J. A., \& Childress, A. E. (2004). Colloidal adhesion to hydrophilic membrane surfaces. Journal of Membrane Science, 241(2), 235-248.

Brant, J. a., Johnson, K. M., \& Childress, A. E. (2006). Examining the electrochemical properties of a nanofiltration membrane with atomic force microscopy. Journal of Membrane Science, 276(1-2), 286-294.

Breite, D., Went, M., Prager, A., \& Schulze, A. (2017). Tailoring Membrane Surface Charges: A Novel Study on Electrostatic Interactions during Membrane Fouling, 2017-2030.

Butt, H. J. (1991). Measuring electrostatic, van der Waals, and hydration forces in electrolyte solutions with an atomic force microscope. Biophysical Journal, 60(December), 1438-1444.

Butt, H. J., Cappella, B., \& Kappl, M. (2005). Force measurements with the atomic force microscope: Technique, interpretation and applications. Surface Science Reports, 59(1-6), 1-152.

Cabib, E., Roh, D. H., Schmidt, M., Crotti, L. B., \& Varma, A. (2001). The Yeast Cell Wall and Septum as Paradigms of Cell Growth and Morphogenesis. Journal of Biological Chemistry, 276(23), 19679-19682.

Calvo, J. I., Hernández, A., Prádanos, P., \& Tejerina, F. (1996). Charge Adsorption and Zeta Potential in Cyclopore Membranes. Journal of Colloid and Interface Science, $181(2), 399-412$.

Carl, P., \& Schillers, H. (2008). Elasticity measurement of living cells with an atomic force microscope: data acquisition and processing. Pflügers Archiv : European 
Journal of Physiology, 457(2), 551-9. http://doi.org/10.1007/s00424-008-0524-3

Castrillón, R.-V. S., Perreault, F., De Faria, A. F., \& Elimelech, M. (2015). Interaction of graphene oxide with bacterial cell membranes: Insights from force spectroscopy. Environmental Science and Technology Letters, 2(4), 112-117.

Chen, J., Hessler, J. a, Putchakayala, K., Panama, B. K., Khan, D. P., Hong, S., ... Orr, B. G. (2009). Cationic nanoparticles induce nanoscale disruption in living cell plasma membranes. The Journal of Physical Chemistry. B, 113(32), 11179-85.

Cleveland, J. P., Manne, S., Bocek, D., \& Hansma, P. K. (1993). A Nondestructive Method for Determining the Spring Constant of Cantilevers for Scanning Force Microscopy. Review of Scientific Instruments, 64, 403-405.

Collins, L., Jesse, S., Kilpatrick, J. I., Tselev, A., Okatan, M. B., Kalinin, S. V., \& Rodriguez, B. J. (2015). Kelvin probe force microscopy in liquid using electrochemical force microscopy. Beilstein Journal of Nanotechnology, 6(1), 201-214.

Costerton, J. W., Ingram, J. M., \& Cheng, K. J. (1974). Structure and function of the cell envelope of gram-negative bacteria. Bacteriological Reviews, 38(1), 87-110.

Craig, W. A. (1998). Pharmacokinetic/Pharmacodynamic Parameters: Rationale for Antibacterial Dosing of Mice and Men. Clinical Infectious Diseases, 26(1), 1-10. http://doi.org/10.1086/516284

Deshrnukh, S. S., \& Childress, A. E. (2001). Zeta potential of commercial RO membranes : influence of source water type and chemistry. Desalination, 4(140), $87-95$.

Fakhrullin, R. F., Zamaleeva, A. I., Morozov, M. V, Tazetdinova, D. I., Alimova, F. K., Hilmutdinov, A. K., ... Culha, M. (2009). Living fungi cells encapsulated in polyelectrolyte shells doped with metal nanoparticles. Langmuir: The ACS Journal of Surfaces and Colloids, 25(8), 4628-34.

Fang, Y., \& Duranceau, S. J. (2013). Study of the effect of nanoparticles and surface morphology on reverse osmosis and nanofiltration membrane productivity. Membranes, 3(3), 196-225. http://doi.org/10.3390/membranes3030196

Fei, E., \& Brock, R. (2013). Park XE-100 AFM User Manual. 
Fievet, P., Szymczyk, A., Labbez, C., Aoubiza, B., Simon, C., Foissy, A., \& Pagetti, J. (2001). Determining the Zeta Potential of Porous Membranes Using Electrolyte Conductivity inside Pores. Journal of Colloid and Interface Science, 235(2), 383-390.

Fletcher, M. (1988). Attachment of Pseudomonas fluorescens to glass and influence of electrolytes on bacterium-substratum separation distance. Journal of Bacteriology, 170(5), 2027-2030.

Gaboriaud, F., \& Dufrêne, Y. F. (2007). Atomic force microscopy of microbial cells: application to nanomechanical properties, surface forces and molecular recognition forces. Colloids and Surfaces. B, Biointerfaces, 54(1), 10-9.

Gardiner, B. S., Wong, K. K. L., Joldes, G. R., Rich, A. J., Tan, C. W., Burgess, A. W., \& Smith, D. W. (2015). Discrete Element Framework for Modelling Extracellular Matrix, Deformable Cells and Subcellular Components. PLoS Computational Biology, 11(10), 1-23.

Gera, D., Gautam, M., Tsuji, Y., Kawaguchi, T., \& Tanaka, T. (1998). Computer Simulation of Bubbles in Large-Particle Fluidized Beds. Power Technology, 98, $38-47$.

Ginzburg, V. V, \& Balijepalli, S. (2007). Modeling the thermodynamics of the interaction of nanoparticles with cell membranes. Nano Letters, 7(12), 3716-22.

Green, C. P., Lioe, H., Cleveland, J. P., Proksch, R., Mulvaney, P., \& Sader, J. E. (2004). Normal and torsional spring constant of atomic force microscope cantilevers cantilevers. Review of Scientific Instruments, 75, 1988-1996. http://doi.org/10.1063/1.1753100

Grierson, D. S., Flater, E. E., \& Carpick, R. W. (2005). Accounting for the JKR-DMT transition in adhesion and friction measurements with atomic force microscopy. Journal of Adhesion Science and Technology, 19(3-5), 291-311.

Han, S. W., Nakamura, C., Obataya, I., Nakamura, N., \& Miyake, J. (2005). A molecular delivery system by using AFM and nanoneedle. Biosensors \& Bioelectronics, 20(10), 2120-5.

Harimawan, A., Zhong, S., Lim, C. T., \& Ting, Y. P. (2013). Adhesion of B. subtilis 
spores and vegetative cells onto stainless steel - DLVO theories and AFM spectroscopy. Journal of Colloid and Interface Science, 405(November 2016), 233-241.

Hermens, J., Canton, H., Janssen, P., \& Jong, R. O. B. D. E. (1984). Quantitative Sturcture- Activity Relationships and Toxicity Studies of Mixtures of Chemcials with Anaesthetic Potency: Acute Lethal and Sublethal Toxicity to Daphnia Magna. Aquatic Toxicology, 5, 143-154.

Hilal, N., Ismail, A. ., \& Wright, C. . (2015). Membrane Fabrication: Principles, Optimization and Applications. (N. Hilal, A. . Ismail, \& C. . Wright, Eds.) (1st editio). CRC Press.

Hunter, R. J. (1989). Electrofield Interfaces: The Electrical Double Layer. In R. J. Hunter (Ed.), Foundations of Colloid Science (V. 2, pp. 395-449). New York: Oxford University Press.

Hurwitz, G., Guillen, G. R., \& Hoek, E. M. V. (2010). Probing polyamide membrane surface charge, zeta potential, wettability, and hydrophilicity with contact angle measurements. Journal of Membrane Science, 349(1-2), 349-357.

Jena, B. ., \& Cho, S.-J. (2002). The Atomic Force Microscope in the Study of Membrane Fusion and Exocytosis. In B. . Jena \& J. K. . Horber (Eds.), Atomic Force Microscopy in Cell Biology; Volumn 68 (1st ed., pp. 33-51).

Johnson, D., Galiano, F., Deowan, S. A., Hoinkis, J., Figoli, A., \& Hilal, N. (2015). Adhesion forces between humic acid functionalized colloidal probes and polymer membranes to assess fouling potential. Journal of Membrane Science, $484,35-46$.

Johnson, D., \& Hilal, N. (2015). Characterisation and quantification of membrane surface properties using atomic force microscopy: A comprehensive review. Desalination, 356, 149-164.

Johnson, R. S. (2012). Integration and Differential Equations.

JPK Instruments. (2009). JPK NanoWizard ${ }^{\circledR}$ II SPM Software Release 3.3a.

Kuhn, S., \& Rahe, P. (2014). Discriminating short-range from van der Waals forces using total force data in noncontact atomic force microscopy. Physical Review B, 
89(23), 235417.

Lau, P. C. Y., Dutcher, J. R., Beveridge, T. J., \& Lam, J. S. (2009). Absolute quantitation of bacterial biofilm adhesion and viscoelasticity by microbead force spectroscopy. Biophysical Journal, 96(7), 2935-2948.

Lee, G. U., Kidwell, D. a, \& Colton, R. J. (1994). Sensing Discrete Streptavidin-Biotin Interactions with Atomic Force Microscopy. Langmuir, 2(9), $354-357$

Lee, S., \& Elimelech, M. (2006). Relating organic fouling of reverse osmosis membranes to intermolecular adhesion forces. Environmental Science and Technology, 40(3), 980-987.

Leroueil, P. R., Berry, S. a, Duthie, K., Han, G., Rotello, V. M., McNerny, D. Q., ... Holl, M. M. B. (2008). Wide varieties of cationic nanoparticles induce defects in supported lipid bilayers. Nano Letters, 8(2), 420-4.

Levy, R., \& Maaloum, M. (2002). Measuring the spring constant of atomic force microscope cantilevers : Thermal fluctuations and other methods Measuring the spring constant of atomic force microscope cantilevers: thermal $\mathrm{R} L$ '. Nanotechnology, 13, 33-37.

Liang, S., Qi, G., Xiao, K., Sun, J., Giannelis, E. P., Huang, X., \& Elimelech, M. (2014). Organic fouling behavior of superhydrophilic polyvinylidene fluoride (PVDF) ultrafiltration membranes functionalized with surface-tailored nanoparticles: Implications for organic fouling in membrane bioreactors. Journal of Membrane Science, 463, 94-101.

Limbach, L. K., Wick, P., Manser, P., Grass, R. N., Bruinink, A., \& Stark, W. J. (2007). Exposure of Engineered Nanoparticles to Human Lung Epithelial Cells: Influence of Chemical Composition and Catalytic Activity on Oxidative Stress. Environmental Science \& Technology, 41(11), 4158-4163.

Lipke, P. N., \& Ovalle, R. (1998). Cell Wall Architecture in Yeast : New Structure and New Challenges MINIREVIEW Cell Wall Architecture in Yeast : New Structure and New Challenges $\uparrow, 180(15), 3735-3740$.

Liu, L., Guo, K., Lu, J., Venkatraman, S. S., Luo, D., Ng, K. C., ... Yang, Y.-Y. (2008). 
Biologically active core/shell nanoparticles self-assembled from cholesterol-terminated PEG-TAT for drug delivery across the blood-brain barrier. Biomaterials, 29(10), 1509-17.

Lobo-Guerrero, S., \& Vallejo, L. E. (2006). Discrete element method analysis of railtrack ballast degradation during cyclic loading. Granular Matter, 8(3-4), $195-204$.

Longo, G., Cnr, M., Longo, G., Rio, L. M., Bizzini, A., Trampuz, A., ... Dietler, G. (2017). Rapid detection of bacterial resistance to antibiotics using AFM cantilevers as nanomechanical sensors Rapid detection of bacterial resistance to antibiotics using AFM cantilevers as nanomechanical sensors, (June 2013).

Lynch, I., \& Dawson, K. A. (2008). The key role of protein-nanoparticle interactions in nanomedicine and, 3(1), 40-47.

Majno, G., \& Joris, I. (1995). Review Apoptosis, Oncosis, and Necrosis An Overview of Cell Death. American Journal of Patbology, 146(1), 3-15.

Marie, J., Ce, F., \& Dague, E. (2013). Use of atomic force microscopy ( AFM ) to explore cell wall properties and response to stress in the yeast Saccharomyces cerevisiae, $c$ (Walker 1998). http://doi.org/10.1007/s00294-013-0411-0

McClanahan, C. (2009). Antifungals.

McKenzie, H., Main, J., Pennington, C. R., \& Parratt, D. (1990). Antibody to selected strains of Saccharomyces cerevisiae (baker's and brewer's yeast) and Candida albicans in Crohn's disease. Gut, 31(5), 536-8.

McNamee, C. E., Pyo, N., \& Higashitani, K. (2006). Atomic force microscopy study of the specific adhesion between a colloid particle and a living melanoma cell: Effect of the charge and the hydrophobicity of the particle surface. Biophysical Journal, 91(5), 1960-9.

McNamee, C. E., Pyo, N., Tanaka, S., Kanda, Y., \& Higashitani, K. (2006). Imaging of a soft, weakly adsorbing, living cell with a colloid probe tapping atomic force microscope technique. Colloids and Surfaces. B, Biointerfaces, 47(1), 85-9.

Mi, B., \& Elimelech, M. (2008). Chemical and physical aspects of organic fouling of forward osmosis membranes. Journal of Membrane Science, 320(1-2), 292-302. 
Muller, D. J., \& Dufrene, Y. F. (2011). Atomic force microscopy: a nanoscopic window on the cell surface, 21(8), 461-469.

Nasti, A., Zaki, N. M., de Leonardis, P., Ungphaiboon, S., Sansongsak, P., Rimoli, M. G., \& Tirelli, N. (2009). Chitosan/TPP and chitosan/TPP-hyaluronic acid nanoparticles: systematic optimisation of the preparative process and preliminary biological evaluation. Pharmaceutical Research, 26(8), 1918-30.

Oatley-Radcliffe, D. ., Aljohani, N., Williams, P. ., \& Hilal, N. (2017). Electrokinetic Phenomena for Membrane Charge. In N. Hilal, A. F. Ismail, T. Matsuura, \& D. L. Oatley-Radcliffe (Eds.), Membrane Characterization. Elsevier.

Oatley, D. L., Llenas, L., Aljohani, N. H. M., Williams, P. M., Martínez-Lladó, X., Rovira, M., \& de Pablo, J. (2013). Investigation of the dielectric properties of nanofiltration membranes. Desalination, 315, 100-106.

Oatley, D. L., Llenas, L., Pérez, R., Williams, P. M., Martínez-Lladó, X., \& Rovira, M. (2012). Review of the dielectric properties of nanofiltration membranes and verification of the single oriented layer approximation. Advances in Colloid and Interface Science, 173, 1-11.

Ojewole, E., Mackraj, I., Naidoo, P., \& Govender, T. (2008). Exploring the use of novel drug delivery systems for antiretroviral drugs. European Journal of Pharmaceutics and Biopharmaceutics : Official Journal of Arbeitsgemeinschaft Für Pharmazeutische Verfahrenstechnik e.V, 70(3), 697-710.

Okazaki, M., Sugita, T., Shimizu, M., Ohode, Y., Iwamoto, K., deVrind-deJong, E. W., ... Corstjens, P. L. A. M. (1997). Partial purification and characterization of manganese-oxidizing factors of Pseudomonas putida GB-1. Applied and Environmental Microbiology, 63(12), 4793-4799.

Pfreundschuh, M., Martinez-martin, D., Mulvihill, E., Wegmann, S., \& Muller, D. J. (2014). Multiparametric high-resolution imaging of native proteins by force-distance curve - based AFM. Nature Protocols, 9(5), 1113-1130.

Polyanin, A. D., \& Manzhirov, A. V. (2006). Elementary Geometry. In Handbook of Mathematics for Engineers and Scientists (pp. 43-76).

Polysciences Inc. (2013). Glass Beads, 1(800), 18976. 
Potthoff, E., Guillaume-Gentil, O., Ossola, D., Polesel-Maris, J., LeibundGut-Landmann, S., Zambelli, T., \& Vorholt, J. A. (2012). Rapid and Serial Quantification of Adhesion Forces of Yeast and Mammalian Cells. PLoS ONE, 7(12).

Puech, P.-H., Taubenberger, A., Ulrich, F., Krieg, M., Muller, D. J., \& Heisenberg, C.-P. (2005). Measuring cell adhesion forces of primary gastrulating cells from zebrafish using atomic force microscopy. Journal of Cell Science, 118(Pt 18), 4199-4206.

Pyo, N., Tanaka, S., McNamee, C. E., Kanda, Y., Fukumori, Y., Ichikawa, H., \& Higashitani, K. (2006). Effect of the cell type and cell density on the binding of living cells to a silica particle: an atomic force microscope study. Colloids and Surfaces. B, Biointerfaces, 53(2), 278-87.

Ramjegathesh, R., \& Vanitha, S. (2014). Bio Control Potential of Pseudomonas fluorescens against Coleus Root Rot Disease. Journal of Plant Pathology \& Microbiology, 05(01), 1-4.

Rogers, K. (2007). Membrane Biology.

Roiter, Y., Ornatska, M., Rammohan, A. R., Balakrishnan, J., Heine, D. R., \& Minko, S. (2008). Interaction of nanoparticles with lipid membrane. Nano Letters, 8(3), $941-4$.

Ruiz-Cabello, F. J. M., Trefalt, G., Maroni, P., \& Borkovec, M. (2014). Electric double-layer potentials and surface regulation properties measured by colloidal-probe atomic force microscopy. Physical Review E - Statistical, Nonlinear, and Soft Matter Physics, 90(1).

Selvakumar, D., Karim, N., Miyamoto, M., Furuichi, Y., \& Komiyama, T. (2006). Recombinant single-chain anti-idiotypic antibody: an effective fungal beta-1,3-glucan synthase inhibitor. Biological \& Pharmaceutical Bulletin, 29(9), 1848-1853. http://doi.org/JST.JSTAGE/bpb/29.1848 [pii]

Shim, Y., Lee, H.-J., Lee, S., Moon, S.-H., \& Cho, J. (2002). Effects of Natural Organic Matter and Ionic Species on Membrane Surface Charge. Environmental Science and Technology, 36(17), 3864-3871. 
Shukla, R., Bansal, V., Chaudhary, M., Basu, A., Bhonde, R. R., \& Sastry, M. (2005). Biocompatibility of Gold Nanoparticles and Their Endocytotic Fate Inside the Cellular Compartment : A Microscopic Overview, (25), 10644-10654.

SimBac. (2013). outer membrane proteins.

Sivasakthi, S., Usharani, G., \& Saranraj, P. (2014). Biocontrol potentiality of plant growth promoting bacteria ( PGPR ) - Pseudomonas fluorescens and Bacillus subtilis : A review, 9(16), 1265-1277.

Sosnik, A., Chiappetta, D. a, \& Carcaboso, A. M. (2009). Drug delivery systems in HIV pharmacotherapy: what has been done and the challenges standing ahead. Journal of Controlled Release: Official Journal of the Controlled Release Society, 138(1), 2-15.

Stoton, A. (2001). The Application of Atomic Force Microscopy in the Characterisation of Membrane Surfaces. University of Wales Swansea.

Sze, A., Erickson, D., Ren, L., \& Li, D. (2003). Zeta-potential measurement using the Smoluchowski equation and the slope of the current-time relationship in electroosmotic flow. Journal of Colloid and Interface Science, 261(2), 402-410.

Tan, H., Ma, B., Li, X., Jian, S., \& Yang, H. (2014). Effect of competitive adsorption between sodium tripolyphosphate and naphthalene superplasticizer on fluidity of cement paste. Journal Wuhan University of Technology, Materials Science Edition, 29(2), 334-340. http://doi.org/10.1007/s11595-014-0917-4

Thomas, T. E., Aani, S. Al, Oatley-Radcliffe, D. L., Williams, P. M., \& Hilal, N. (2016). Laser Doppler Electrophoresis and Electro-osmotic Flow Mapping: a novel methodology for the determination of membrane surface zeta potential. Journal of Membrane Science, 523(October 2016), 524-532.

Torii, A., Sasaki, M., Hane, K., \& Okuma, S. (1996). A method for determining the spring constant of cantilevers for atomic force microscopy, 7, 179-184.

Touhami, A., Hoffmann, B., Vasella, A., Denis, F. a., \& Dufrêne, Y. F. (2003). Aggregation of yeast cells: Direct measurement of discrete lectin-carbohydrate interactions. Microbiology, 149(10), 2873-2878.

Turner, R. D., Hurd, A. F., Cadby, A., Hobbs, J. K., \& Foster, S. J. (2013). Cell wall 
elongation mode in Gram-negative bacteria is determined by peptidoglycan architecture. Nature Communications, 4, 1496.

Vasir, J. K., \& Labhasetwar, V. (2008). Quantification of the force of nanoparticle-cell membrane interactions and its influence on intracellular trafficking of nanoparticles. Biomaterials, 29(31), 4244-52.

Verwey, E. J. W., \& Overbeek, J. T. G. (1948). Theory of a Single Double Layer. In Theory of the Stability of Lyophobic Colloids (pp. 22-50). New York: Elsevier Publishing Company, inc.

Vrijenhoek, E. M., Hong, S., \& Elimelech, M. (2001). Influence of membrane surface properties on initial rate of colloidal fouling of reverse osmosis and nanofiltration membranes. Journal of Membrane Science, 188(1), 115-128.

Waite, T. D., \& Wen, X. (2011). Combined effect of membrane and foulant hydrophobicity and surface charge on adsorptive fouling during ... Journal of Membrane Science, 373(1-2), 140-151.

Wilhelm, C., Gazeau, F., Roger, J., Pons, J. N., Bacri, J., \& Curie, M. (2002). Interaction of Anionic Superparamagnetic Nanoparticles with Cells: Kinetic Analyses of Membrane Adsorption and Subsequent Internalization, (12), 81488155.

Williams, V., \& Fletcher, M. (1996). Pseudomonas fluorescens adhesion and transport through porous media are affected by lipopolysaccharide composition . These include: Pseudomonas fluorescens Adhesion and Transport through Porous Media Are Affected by Lipopolysaccharide Composition, 62(1), 100-104.

Wojcikiewicz, E. P., Zhang, X., \& Moy, V. T. (2004). Force and Compliance Measurements on Living Cells Using Atomic Force Microscopy (AFM). Biol. Proced. Online, 6(1), 1-9.

Wright, B. M., \& Revenko, I. (2004). TappingMode Atomic Force Microscopy Operation in Fluid. Veeco Solutions for a Nanoscale World.

Wright, C. J., Shah, M. K., Powell, L. C., \& Armstrong, I. (2010). Application of AFM from microbial cell to biofilm. Scanning, 32(3), 134-149.

Xiong, Z., Zhang, M., \& Ma, M. (2016). Emulsifying properties of ovalbumin: 
Improvement and mechanism by phosphorylation in the presence of sodium tripolyphosphate. Food Hydrocolloids, 60, 29-37. http://doi.org/10.1016/j.foodhyd.2016.03.007

Yap, F. L., \& Zhang, Y. (2007). Protein and cell micropatterning and its integration with micro/nanoparticles assembly. Biosensors \& Bioelectronics, 22(6), 775-88.

Yeh, M., Cheng, K., Hu, C., Huang, Y., \& Young, J. (2011). Novel protein-loaded chondroitin sulfate-chitosan nanoparticles: preparation and characterization. Acta Biomaterialia, 7(10), 3804-12.

Zetasizer. (n.d.). Zeta Potential theory. Zetasizer Technologies, 1-14.

Zhang, D., Chen, X. Q., Wang, Y., Zhang, F. H., \& Gan, Y. (2014). Relationship between asperity-mediated surface forces and topography alteration of silica microspheres sliding on mica, sapphire, and glass substrates under ambient conditions: Atomic force microscopy and theoretical studies. Langmuir, 30(13), $3729-3740$.

Zhang, W., Stack, A. G., \& Chen, Y. (2011). Interaction force measurement between E. coli cells and nanoparticles immobilized surfaces by using AFM. Colloids and Surfaces B: Biointerfaces, 82(2), 316-324.

Zhao, Y., Xing, W., Xu, N., \& Wong, F. S. (2005). Effects of inorganic electrolytes on zeta potentials of ceramic microfiltration membranes. Separation and Purification Technology, 42(2), 117-121.

Zhou, Y., Xu, Y.-B., Xu, J.-X., Zhang, X.-H., Xu, S.-H., \& Du, Q.-P. (2015). Combined Toxic Effects of Heavy Metals and Antibiotics on a Pseudomonas fluorescens Strain ZY2 Isolated from Swine Wastewater. International Journal of Molecular Sciences, 16(2), 2839-2850. 


\section{Appendix}

Appendix.1. Hexadecane and Cell Aggregation Error Plot of Optical Density

Appendix.1.1. Hexadecane Effects on Spectrophotometer Measurement

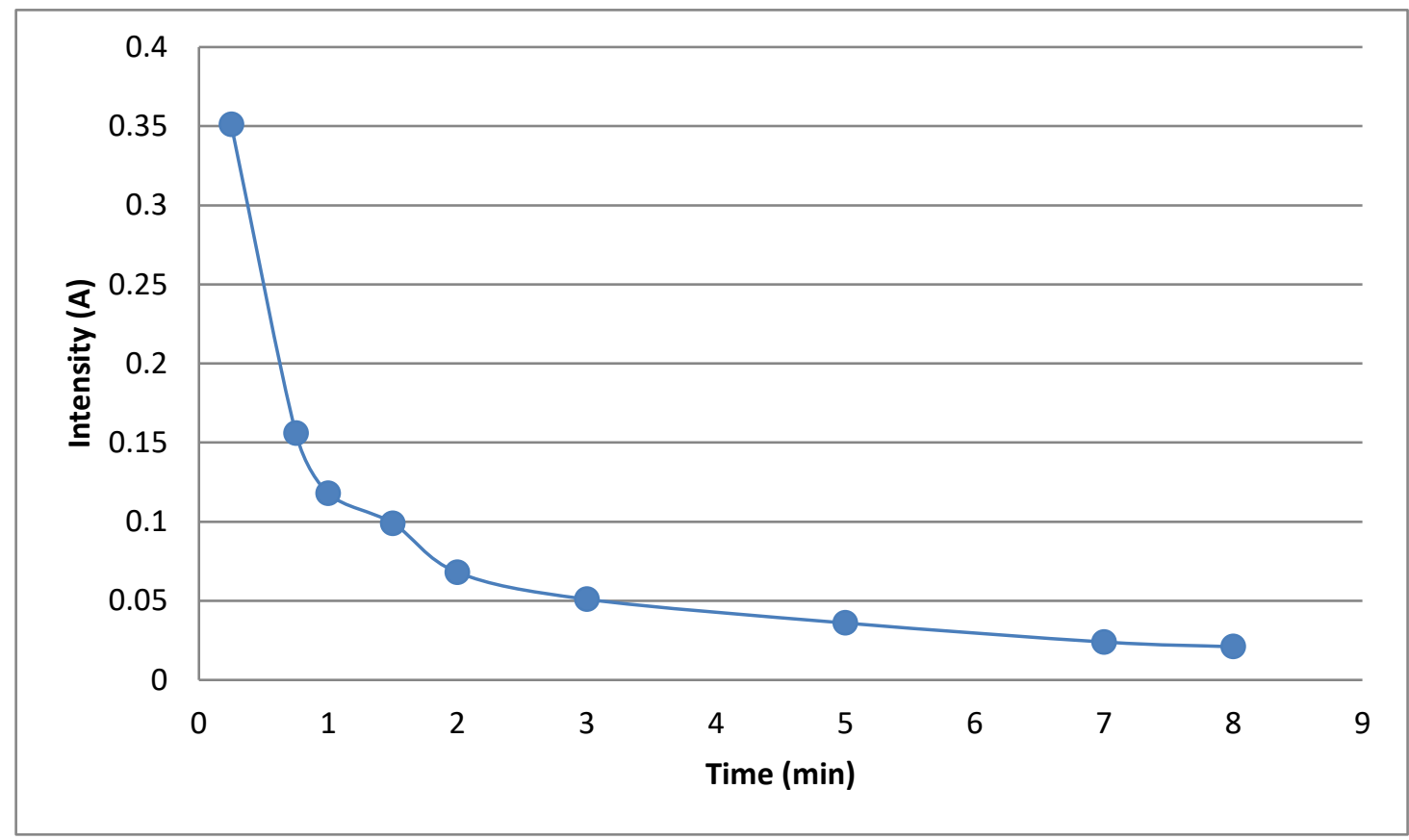

Appendix.1.2. NCYC-1324 \&1681 Cell Aggregation Effects on

Spectrophotometer Measurement

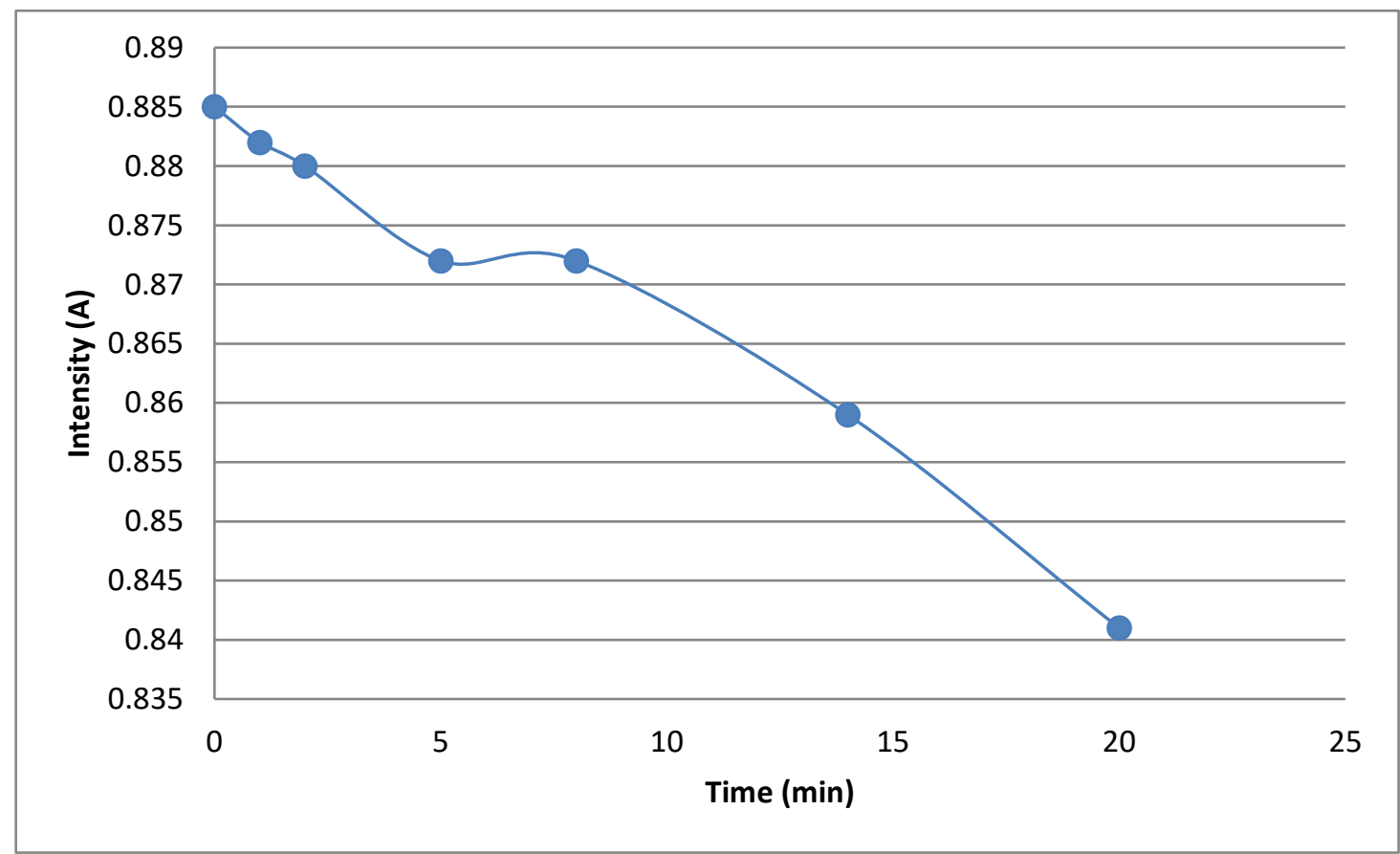


Appendix.1.3. P. fluorescens Cell Aggregation Effects on Spectrophotometer

Measurement

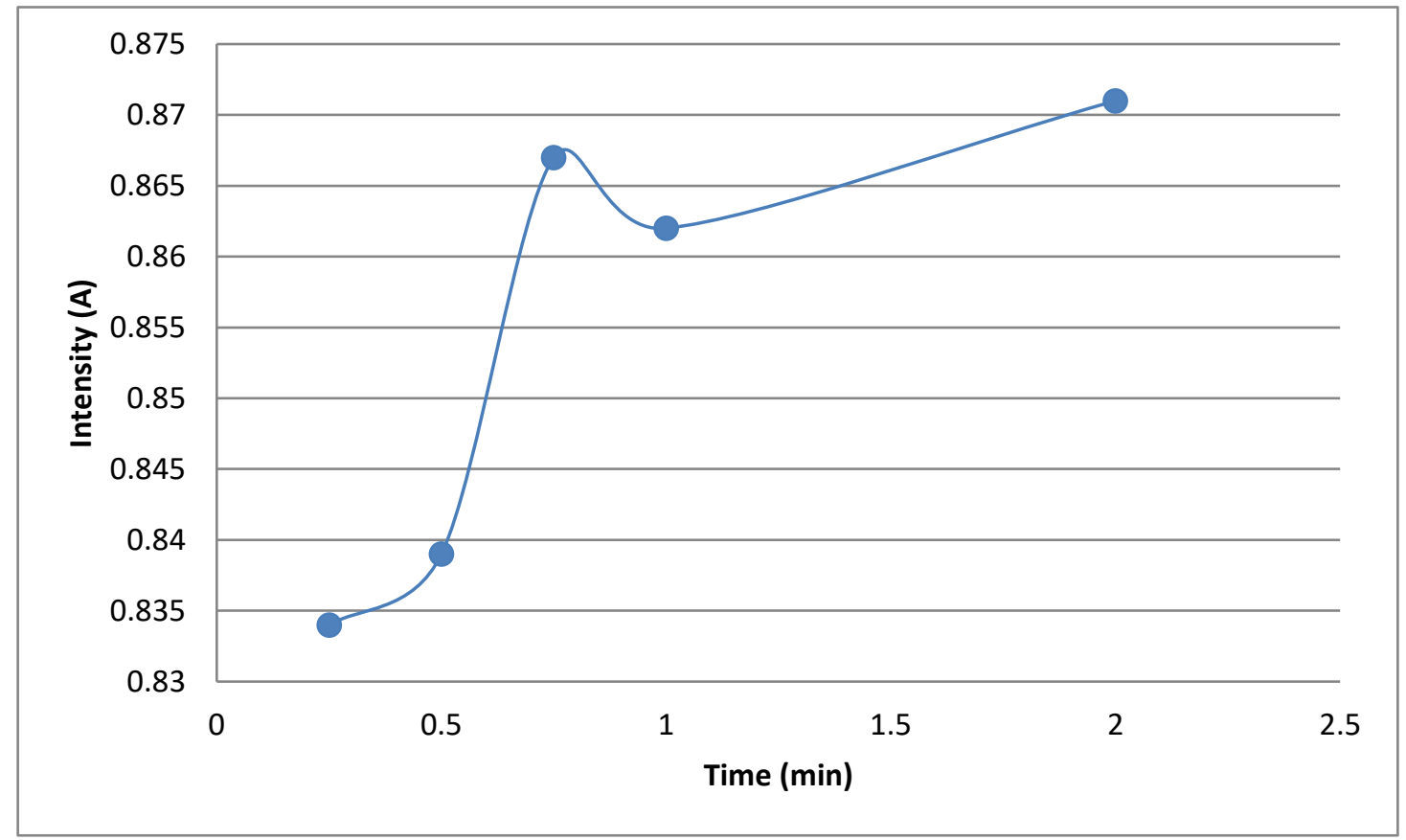


Appendix.2. FORTRAN Code of Data Processing

!===================OVERALL ANALYSIS OF AFM FORCE

CURVE============================

! OVERALL ANALYSIS OF FORCE CURVE FOR ZETA POTENTIAL AND

ADHESION INFORMATION

!

$!$

!==================================MAIN

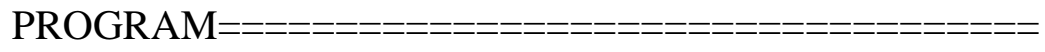

PROGRAM MAIN

IMPLICIT DOUBLE PRECISION (A-Z)

INTEGER*4 DIM,J,K,I,FN,AP,N,B,TL,TZ,TURN1,RY,RZ,DEFINE

PARAMETER $(\mathrm{DIM}=100000)$

DIMENSION

DISTANCEXP(0:DIM),SIGNALXP(0:DIM),TRACE(0:DIM),TRACESIGNAL(0:DI M),DISTANCEXPP(0:DIM),SIGNALXPP(0:DIM),RETRACE(0:DIM),RETRACES IGNAL(0:DIM),TREATEDTRACE(0:DIM),TREATEDTRACEFORCE(0:DIM),TDI STANCE(0:DIM),TW2(0:DIM),TREATEDRETRACE(0:DIM),TREATEDRETRAC EFORCE(0:DIM),POINT2(0:DIM),RDISTANCE(0:DIM),RW2(0:DIM),RFORCEX $\mathrm{P}(0: \mathrm{DIM}), \mathrm{RDIFF} 1(0: \mathrm{DIM})$

INTEGER IOS

CHARACTER DATAVAL

CHARACTER C

CHARACTER(20) :: FNAME,FNAMES,FNAMESS,FNAMESSS

$!=====$ KEYBOARD INPUT $===========$

!-----FILE NUMBER

WRITE $(*, 1100)$ 
1100 FORMAT $(/$, FILE NUMBER = ',l)

$\operatorname{READ}(*, *)$ FN

!-----PARTICLE RADIUS

WRITE $(*, 1200)$

1200 FORMAT $(/$, 'PARTICLE RADIUS [micron] = ',l)

$\mathbf{R E A D}(*, *)$ RR

!-----PARTICLE ZETA POTENTIAL

WRITE $(*, 1300)$

1300 FORMAT $(/$, PARTICLE ZETA $[\mathrm{mV}]=$ ',,$)$

$\operatorname{READ}(*, *)$ PZETA

!-----ZETA POTENTIAL ESTIMATION ACCURACY POINT

WRITE $(*, 1400)$

1400 FORMAT(/,'SURFACE DETECT LENGTH = ',।)

$\operatorname{READ}(*, *) \mathrm{SX}$

!-----ZETA POTENTIAL ESTIMATION ACCURACY POINT-

WRITE $(*, 1500)$

1500 FORMAT(/,'SURFACE BENDING HEIGHT = ', $)$

$\mathbf{R E A D}(*, *)$ SY

!-----IONIC STRENGTH

WRITE $(*, 1600)$

1600 FORMAT $(/$, IONIC STRENGTH $[\mathrm{mol} / \mathrm{L}]=$ = ',l)

$\operatorname{READ}(*, *)$ IONSTER

!-----CANTILEVER SPRING CONSTANT-

WRITE $(*, 1700)$

1700 FORMAT(/,'CANTILEVER SPRING CONSTANT $[\mathrm{N} / \mathrm{m}]=$ = ',l)

READ(*,*) SPRING

!----ZETA POTENTIAL ESTIMATION ACCURACY POINT

WRITE $(*, 1800)$

1800 FORMAT(/,'ACCURACY POINT = ',l)

$\operatorname{READ}(*, *)$ AP 
!=====TRACE DOWN FORCE CURVE READ=====

$\mathrm{FC}=\mathrm{FN}-1$

DO $\mathrm{J}=1, \mathrm{FN}$

WRITE(FNAME,'(I2)')J

OPEN (UNIT=3,FILE='J:|bacterialPF STP 10-2 pH9 hydrocylapetite forcel'//TRIM(ADJUSTL(FNAME))//'.txt',STATUS='OLD')

DO $\mathrm{H}=1,72$

$\operatorname{READ}(3, *) \mathrm{C}$

END DO

DO I=1,DIM

$\operatorname{READ}\left(3,{ }^{*}\right.$, IOSTAT=IOS $)$ DISTANCEXP(I), SIGNALXP(I)

IF $(\mathrm{IOS} /=0)$ THEN

$\mathrm{N}=\mathrm{I}-1$

EXIT

END IF

END DO

CLOSE(3)

!=====UPTAKE TRACE DOWN CURVE=========

NUMBER $=\mathrm{N}$

DO $\mathrm{I}=1, \mathrm{~N}$

TRACE $(\mathrm{I})=$ DISTANCEXP(I)

TRACESIGNAL(I)=SIGNALXP(I)

END DO

!=====RETRACE FORCE CURVEREAD========== 
$\mathrm{A}=\mathrm{NUMBER}+137$

WRITE(FNAMES,'(I2)')J

OPEN (UNIT=4,FILE='J:Ibacteria\PF STP 10-2 pH9 hydrocylapetite forcel'//TRIM(ADJUSTL(FNAMES))//'.txt',STATUS='OLD')

DO $\mathrm{H}=1, \mathrm{~A}$

$\operatorname{READ}(4, *) \mathbf{C}$

END DO

DO I=1,DIM

READ( $4,{ }^{*}$, IOSTAT=IOS) DISTANCEXPP(I),SIGNALXPP(I)

IF $(\mathrm{IOS} /=0)$ THEN

$\mathrm{B}=\mathrm{I}-1$

EXIT

END IF

END DO

CLOSE(4)

!=====UPTAKE RETRACE CURVE=========

DO $\mathrm{I}=1, \mathrm{~B}$

RETRACE $(\mathrm{I})=$ DISTANCEXPP $(\mathrm{I})$

RETRACESIGNAL(I)=SIGNALXPP(I)

END DO

$!=====$ CONSTACT INPUT AND UNITS SYNC=============

$\mathrm{PI}=\mathrm{DACOS}(-1 \mathrm{D} 0)$

!---Boltzmann constant in $\mathrm{J} / \mathrm{K}$ 
$\mathrm{BOLTZC}=1.38 \mathrm{D}-23$

!---Avogadro constant in $1 / \mathrm{mol}$

AVOGADRO $=6.0225 \mathrm{D} 23$

!---Temperature in Kelvin $(=25 \mathrm{oC})$

$\mathrm{TEMP}=298.15 \mathrm{D} 0$

!---Electric field constant in $\mathrm{C} / \mathrm{V} / \mathrm{m}$

ELEFIELDC $=8.854 \mathrm{D}-12$

!---Elemental electron charge in $\mathrm{J} / \mathrm{V}$

CHARGE $=1.602 \mathrm{D}-19$

!---Dielectric constant for water at $25 \mathrm{oC}$

DIELEC $=78.55 \mathrm{D} 0$

!=========MV TO V=============

PZETA $=$ PZETA $/ 1000$

!=====CALL SUBROUTINE===========================

! LINE:1

!---NANO-PARTICLE SURFACE CHARGEESTIMATION

CALL NPSCESMT

(CHARGE,AVOGADRO,IONSTER,DIELEC,ELEFIELDC,BOLTZC,TEMP,PZET

A,SIGMA1)

!---TRACE DOWN FORCE CURVE ZERO AXIS AND MINIMIZE BENDING

CALL TDFCZAMB

(N,TRACE,TRACESIGNAL,SPRING,RR,TREATEDTRACE,TREATEDTRACEF

ORCE)

!---RETRACE FORCE CURVE ZETO AXIS AND MINIMIZE BENDING

CALL RTFCZAMB

(B,RETRACE,RETRACESIGNAL,SPRING,RR,TREATEDRETRACE,TREATEDR 
ETRACEFORCE)

$!$

!LINE:2

!---TRACE DOWN FORCE ANALYSIS

CALL TDFA

(PI,N,RR,TREATEDTRACE,TREATEDTRACEFORCE,TL,TZ,TW2,TE,TPENAT RATIONMAX)

!---SURFACE BENDING CALCULATION

CALL SBCAL (SX,SY,SR)

$!$

$!$

!LINE:3

!---ZETA POTENTIAL ANALYSIS

CALL ZPA (TL,RR,IONSTR,AP,SIGMA1,TDISTANCE,TW2,ZETA)

!---SURFACE RATIO CALCULATION OF ZETA POTENTIAL ANALYSIS

CALL SRCALOZPA (AP,IONSTR,SR,RR,RATIO)

!---AFM RETRACE ADHESION ANALYSIS

CALL AFMRTAA

(K,B,RR,TREATEDRETRACE,TREATEDRETRACEFORCE,POINT2,RPENATR ATIONMAX,RE,TURN1,RY,RZ,DEFINE,RDISTANCE,RW2,RFORCEXP,MINRF ORCE)

ZETAPOTENTIAL = ZETA $*$ RATIO 
!=====RESULTS WRITE UP===========

!--------TRACE DOWN FORCE CURVE \& ENERGY

WRITE(FNAMES,'(I2)')J

OPEN (UNIT=5,FILE='J:Ibacteria\PF STP 10-2 pH9 hydrocylapetite forcelTDAAINDEXITDAAINDEX'//TRIM(ADJUSTL(FNAMES))//'.txt',STATUS=' OLD')

WRITE $\left(5,{ }^{*}\right)$ 'TRACE DOWN MEAN ELASTIC CONSTANT=', TE

WRITE $(5, *)$ 'MAX PENATRATION=',TPENATRATIONMAX

WRITE $\left(5,{ }^{*}\right)$ 'ZETA POTENTIAL=',ZETAPOTENTIAL

WRITE $\left(5,{ }^{*}\right)$ 'FILE NUMBER=',FN

CLOSE(5)

!--------RETRACE PROPERTIES RESULTS-

WRITE(FNAMES,'(I2)')J

OPEN (UNIT=7,FILE='J: Ibacteria \PF STP 10-2 pH9 hydrocylapetite forcelRTAAINDEXIRTAAINDEX'//TRIM(ADJUSTL(FNAMES))//'.txt',STATUS=' OLD')

WRITE $\left(7,{ }^{*}\right)$ 'RETRACE MEAN ELASTIC CONSTANT=', RE

WRITE $\left(7,{ }^{*}\right)$ 'MAX PENATRATION=',RPENATRATIONMAX

WRITE $(7, *)$ 'MAX ADHESION=',MINRFORCE

WRITE $(7, *)$ 'FILE NUMBER=',FN

CLOSE(7)

!=====WRITE RETRACE DMT================

WRITE(FNAMESS,'(I2)')J

OPEN (UNIT=7,FILE='J: Ibacteria\PF STP 10-2 pH9 hydrocylapetite forcelRTAAIPOTENTIAL'//TRIM(ADJUSTL(FNAMESS))//'.txt',STATUS='OLD') DO I=RY,RZ 
WRITE(7,7500) RDISTANCE(I),RW2(I),RFORCEXP(I)

!,(RDIFF1(I-1)*RDIFF1(I))

\section{END DO}

CLOSE(7)

!=====WRITE ADHESION POINTS================

WRITE(FNAMESSS,'(I2)')J

OPEN (UNIT=8,FILE='J:।bacteria|PF STP 10-2 pH9 hydrocylapetite forcelPOINT\POINT'//TRIM(ADJUSTL(FNAMESSS))//'.txt',STATUS='OLD')

IF (DEFINE.EQ.0) THEN

WRITE $\left(8,^{*}\right)$ 'NO SIGNIFICANT ADHESION'

ELSE IF (DEFINE.EQ.1) THEN

DO $\mathrm{K}=1$,TURN1

WRITE(8,7500) POINT2(K)

END DO

END IF

CLOSE(8)

END DO

7500

FORMAT(1X,T2,E15.8,T20,E15.8,T38,E15.8,T56,E15.8,T74,E15.8,T92,E15.8,T11 0,E15.8,T118,E15.8)

\section{STOP}

END PROGRAM

$!==================$ END OF MAIN

PROGRAM $========================$ 


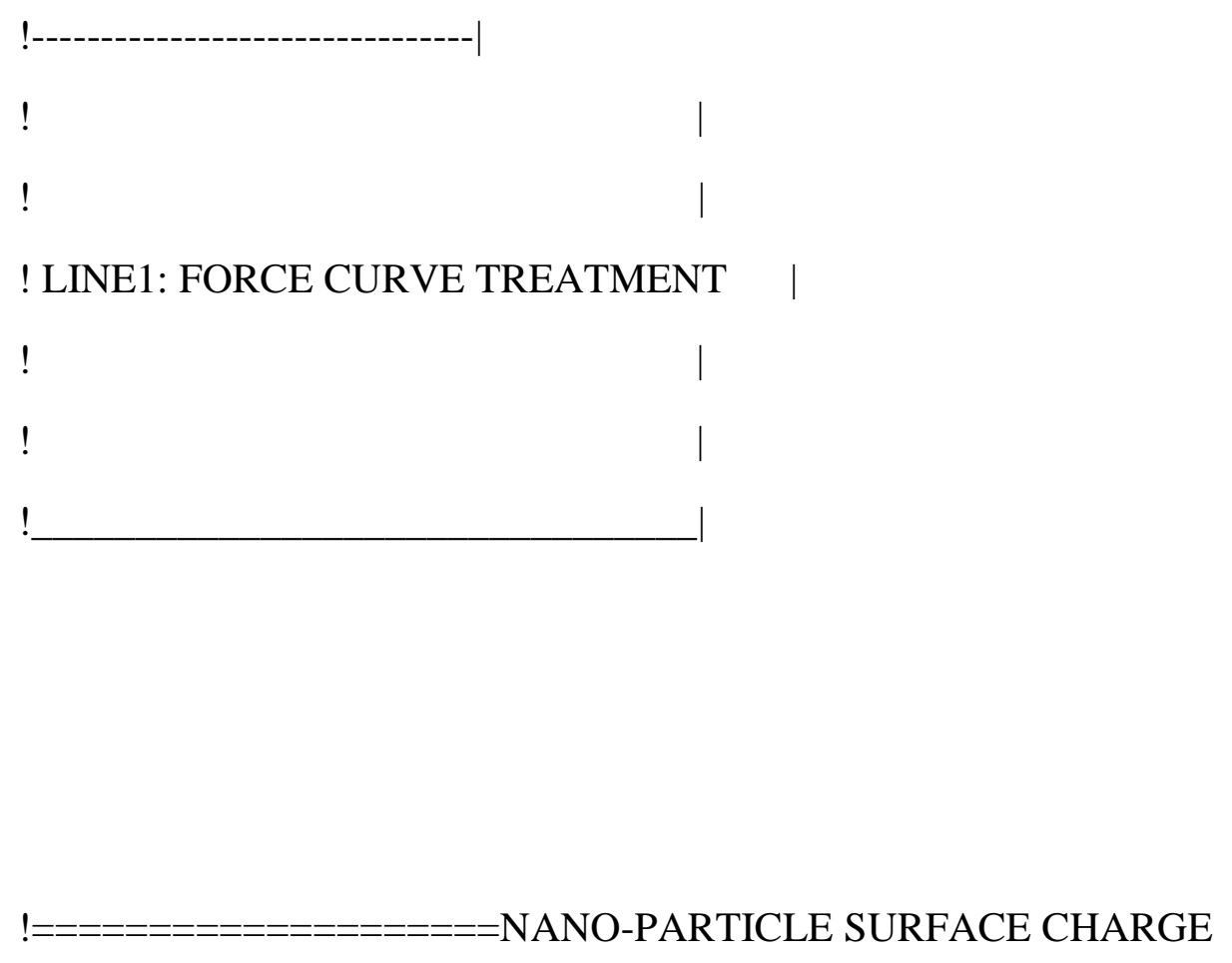

ESTIMATION=====================

SUBROUTINE NPSCESMT

(CHARGE,AVOGADRO,IONSTER,DIELEC,ELEFIELDC,BOLTZC,TEMP,PZET A,SIGMA1)

IMPLICIT DOUBLE PRECISION (A-Z)

INTEGER *4 DIM,N

PARAMETER $(\mathrm{DIM}=100000)$

!========+CALCULATION=========

KAPPA $=(((C H A R G E * 2) * A V O G A D R O * 2000 *$ IONSTER $) /(D I E L E C * E L E F I E L D C$ *BOLTZC*TEMP))**0.5

$\mathrm{SH}=\operatorname{SINH}((\mathrm{CHARGE} * \mathrm{PZETA}) /(2 \mathrm{D} 0 *$ BOLTZC $*$ TEMP $))$

SIGMA1 $=((4 \mathrm{D} 3 *$ AVOGADRO $*$ IONSTER $*$ CHARGE* $(\mathrm{SH})) /(\mathrm{KAPPA}))$

\section{CLOSE(7)}

\section{RETURN}


END SUBROUTINE

!============TRACE DOWN FORCE CURVE ZERO AXIS AND MINIMIZE BENDING

SUBROUTINE TDFCZAMB

(N,TRACE,TRACESIGNAL,SPRING,RR,TREATEDTRACE,TREATEDTRACEF ORCE)

IMPLICIT DOUBLE PRECISION (A-Z)

INTEGER $* 4$ DIM,I,N,J,FN,M

PARAMETER $(\mathrm{DIM}=100000)$

DIMENSION

TRACE(0:DIM),TRACESIGNALL(0:DIM),TRACERAW(0:DIM),TRACESIGNAL (0:DIM),TRACESIGNALDIST(0:DIM),TRACEDIST(0:DIM),DISTANCE(0:DIM), TRACEFORCE(0:DIM),DS(0:DIM),DD(0:DIM),TRACEDIFF(0:DIM),TREATEDT RACE(0:DIM),TREATEDTRACEFORCE(0:DIM)

$!=====$ KEYBOARD INPUT $=====$

!=====FIND MAX RAW DISTANCE======

MAXTRACE $=-10$

DO $\mathrm{I}=1, \mathrm{~N}$

IF (TRACE(I).GT.MAXTRACE) THEN

MAXTRACE $=$ TRACE $(\mathrm{I})$

END IF

END DO

!=====FIT DISTANCE UNITS====== 
DO $\mathrm{I}=1, \mathrm{~N}$

IF (MAXTRACE.LT.10) THEN

TRACERAW $(\mathrm{I})=$ TRACE $(\mathrm{I})$

ELSE IF (MAXTRACE.GT.10) THEN

TRACERAW $(\mathrm{I})=\mathrm{TRACE}(\mathrm{I}) / 1000$

END IF

END DO

!=====ZERO SIGNAL========

DO $\mathrm{I}=1, \mathrm{~N}$

TRACESIGNALERR=TRACESIGNAL(1)

TRACESIGNALL(I)=TRACESIGNAL(I)-TRACESIGNALERR

END DO

$!=====$ FIND MIN FORCE POSITION=========

MINTRACESIGNAL $=10$

DO $\mathrm{I}=1, \mathrm{~N}$

IF (TRACESIGNALL(I).LT.TRACEMINSIGNAL) THEN

MINTRACESIGNAL=TRACESIGNALL(I)

TRACEDISTANCEMINSIGNAL=TRACERAW(I)

END IF

END DO

!=====FIND POINT FOR SLOPING=========

DO $\mathrm{I}=1, \mathrm{~N}$

TRACEDIFF $(\mathrm{I})=($ TRACESIGNALL $(\mathrm{I})-\mathrm{MINTRACESIGNAL})$

MAXTRACEDIFF=(TRACESIGNALL(N)-MINTRACESIGNAL)

IF (TRACEDIFF(I).GT.MAXTRACEDIFF) THEN 
MAXTRACEDIFF=TRACEDIFF(I)

END IF

IF (TRACEDIFF(I).GT.(0.8*MAXTRACEDIFF)) THEN

$\mathrm{M}=\mathrm{I}$

EXIT

END IF

END DO

$!=====$ FIND SLOPE $=====$

DO I=M,N

TRACESLOPE=-(TRACESIGNALL(N)-TRACESIGNALL(M))/(TRACERAW(N)TRACERAW(M))

END DO

!=====REMOVE CANTILEVER BENDING=========

DO $\mathrm{I}=1, \mathrm{~N}$

TRACESIGNALDIST(I)=TRACESIGNALL(I)/TRACESLOPE

TRACEDIST $(\mathrm{I})=$ TRACERAW $(\mathrm{I})+($ TRACESIGNALDIST $(\mathrm{I}))$

END DO

!======ZERO DISTANCE====================

MINTRACEDIST $=10$

DO $\mathrm{I}=1, \mathrm{~N}$

IF (TRACEDIST(I).LT.MINTRACEDIST) THEN

MINTRACEDIST $=$ TRACEDIST(I)

END IF 
END DO

$\begin{aligned} !==== & \text { CONVERT SIGNAL TO FORCE }======= \\ & \text { DO I=1,N } \\ & \text { TREATEDTRACE }(\mathrm{I})=\text { TRACEDIST }(\mathrm{I})-\text { MINTRACEDIST }\end{aligned}$

TREATEDTRACEFORCE(I)=TRACESIGNALDIST(I)*SPRING*1000000/(RR)

END DO

$\mathrm{D}=1$

$\mathrm{E}=1$

RETURN

END SUBROUTINE

!==========END OF SUBROUTINE====================

!===========-RETRACE FORCE CURVE ZERO AXIS AND MINIMIZE

BENDING

SUBROUTINE RTFCZAMB

(B,RETRACE,RETRACESIGNAL,SPRING,RR,TREATEDRETRACE,TREATEDR ETRACEFORCE)

IMPLICIT DOUBLE PRECISION (A-Z)

INTEGER *4 DIM,I,B,J,FN,L

PARAMETER $(\mathrm{DIM}=100000)$

DIMENSION

RETRACE(0:DIM),RETRACESIGNAL(0:DIM),RETRACESIGNALL(0:DIM),RET RACERAW(0:DIM),RETRACESIGNALDIST(0:DIM),RETRACEDIST(0:DIM),TR EATEDRETRACEFORCE(0:DIM),DS(0:DIM),TREATEDRETRACE(0:DIM),RET 
RACEDIFF(0:DIM)

$!=====$ READ FILE $==========$

!=====FIND MAX RAW RETRACE======

MAXRETRACE $=-10$

DO $\mathrm{I}=1, \mathrm{~B}$

IF (RETRACE(I).GT.MAXRETRACE) THEN

MAXRETRACE=RETRACE(I)

END IF

END DO

!=====FIT RETRACE UNITS======

DO $\mathrm{I}=1, \mathrm{~B}$

IF (MAXRETRACE.LT.10) THEN

RETRACERAW $(\mathrm{I})=\operatorname{RETRACE}(\mathrm{I})$

ELSE IF (MAXRETRACE.GT.10) THEN

$\operatorname{RETRACERAW}(\mathrm{I})=\operatorname{RETRACE}(\mathrm{I}) / 1000$

END IF

END DO

!=====ZERO SIGNAL $========$

DO I=1,B

RETRACESIGNALERR=RETRACESIGNAL(B)

RETRACESIGNALL(I)=RETRACESIGNAL(I)-RETRACESIGNALERR END DO

$!=====$ FIND MIN FORCE POSITION========= 


\section{MINRETRACESIGNAL $=10$}

DO $\mathrm{I}=1, \mathrm{~B}$

IF (RETRACESIGNALL(I).LT.MINRETRACESIGNAL) THEN

MINRETRACESIGNAL=RETRACESIGNALL(I)

RETRACDISTEMINSIGNAL=RETRACERAW(I)

END IF

END DO

$!=====$ FIND POINT FOR SLOPING=========

DO $\mathrm{I}=1, \mathrm{~B}$

RETRACEDIFF $(\mathrm{I})=($ RETRACESIGNALL $(\mathrm{I})-\mathrm{MINRETRACESIGNAL})$

MAXRETRACEDIFF=(RETRACESIGNALL(1)-MINRETRACESIGNAL)

IF (RETRACEDIFF(I).GT.MAXRETRACEDIFF) THEN

MAXRETRACEDIFF=RETRACEDIFF(I)

END IF

IF (RETRACEDIFF(I).LT.(0.8*MAXRETRACEDIFF)) THEN

$\mathrm{L}=\mathrm{I}$

EXIT

END IF

END DO

$!=====$ FIND SLOPE $=====$

DO I= $1, \mathrm{~L}$

RETRACESLOPE=-(RETRACESIGNALL(1)-RETRACESIGNALL(L))/(RETRAC ERAW(1)-RETRACERAW(L))

END DO 
!=====REMOVE CANTILEVER BENDING=========

DO $\mathrm{I}=1, \mathrm{~B}$

RETRACESIGNALDIST(I)=RETRACESIGNALL(I)/RETRACESLOPE

RETRACEDIST $(\mathrm{I})=$ RETRACERAW(I)+(RETRACESIGNALDIST(I))

END DO

!======ZERO RETRACE $====================$

MINRETRACEDIST $=10$

DO $\mathrm{I}=1, \mathrm{~B}$

IF (RETRACEDIST(I).LT.MINRETRACEDIST) THEN

MINRETRACEDIST=RETRACEDIST(I)

END IF

END DO

$!======$ CONVERT SIGNAL TO FORCE========

DO $\mathrm{I}=1, \mathrm{~B}$

TREATEDRETRACE(I)=RETRACEDIST(I)-MINRETRACEDIST

TREATEDRETRACEFORCE(I)=RETRACESIGNALDIST(I)*SPRING*1000000/R $\mathrm{R}$

END DO

RETURN

END SUBROUTINE

$==================$ END OF SUBROUTINE======================== 


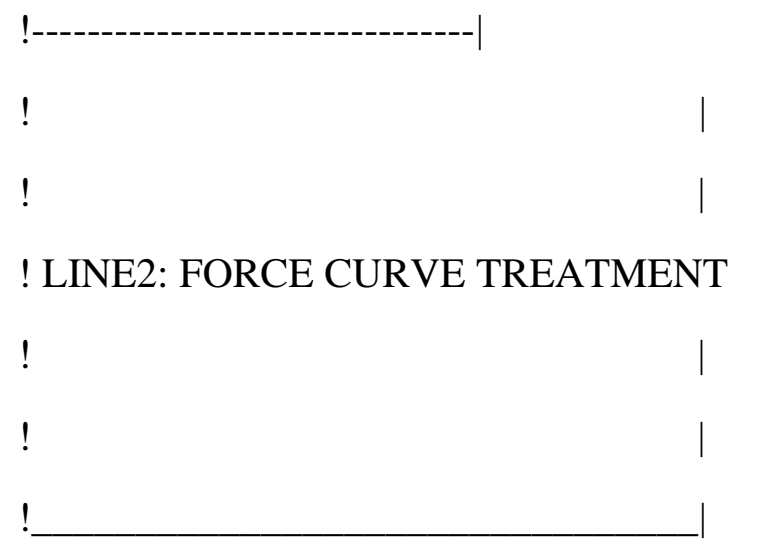

!=ニ=ニ=ニ=ニ====TRACE DOWN FORCE ANALYSIS===ニ==ニ====

AFM TRACE DOWN ADHESION

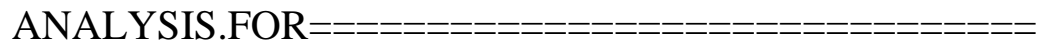

!JKR AND DMT MODEL APPLIED

!=ニ=ニ=ニ=ニ=ニ=ニ=ニ=ニ=ニ=ニ=ニ=ニ=ニ=MAIN

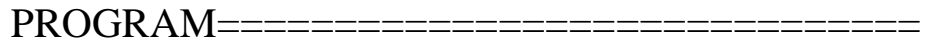

SUBROUTINE TDFA

(PI,N,RR,TREATEDTRACE,TREATEDTRACEFORCE,TL,TZ,TW2,TE,TPENAT RATIONMAX)

IMPLICIT DOUBLE PRECISION (A-Z)

INTEGER*4 I,N,J,DIM,FN,TM,FC,TL,TZ,TS

PARAMETER $(\mathrm{DIM}=100000)$

DIMENSION

TREATEDTRACE(0:DIM),TFORCEXP(0:DIM),TFORCEXPP(0:DIM),TDISTANC E(0:DIM),TA(0:DIM),TPENATRATION(0:DIM),THERTZ(0:DIM),TFLATPUNCH (0:DIM),TW1(0:DIM),ERROR(0:DIM),EXTENDEDTFORCE(0:DIM),TW2(0:DIM) ,TDIFF(0:DIM),TREATEDTRACEFORCE(0:DIM),TFORCEXPPPP(0:DIM),TDIS TANCEXPP(0:DIM) 
! RADIUS IN M

$\mathrm{R}=\mathrm{RR} / 2000000$

! ADD RADIUS EFFECTS

DO $\mathrm{I}=1, \mathrm{~N}$

TFORCEXPP(I)=TREATEDTRACEFORCE $(\mathrm{I}) * \mathrm{RR} / 1000000$

END DO

!=====MIN\&MAX FORCE CALC======================

DO $\mathrm{I}=2,(\mathrm{~N}+17)$

IF (I.GT.N) THEN

TFORCEXPP(I)=TFORCEXPP $(\mathrm{N})$

TREATEDTRACEFORCE(I)=TREATEDTRACEFORCE(I)

END IF

TFORCEXPP $(0)=$ TFORCEXPP $(1)$

TFORCEXPPPP $(1)=($ TFORCEXPP $(0)+$ TFORCEXPP $(1)+$ TFORCEXPP $(2)+$ TFORC EXPP(3)+TFORCEXPP(4)+TFORCEXPP(5)+TFORCEXPP(6)+TFORCEXPP(7)+T FORCEXPP(8)+TFORCEXPP(9)+TFORCEXPP(10)+TFORCEXPP(11)+TFORCEX PP(12)+TFORCEXPP(13)+TFORCEXPP(14)+TFORCEXPP(15)+TFORCEXPP(16) +TFORCEXPP(17)+TFORCEXPP(18)+TFORCEXPP(19))/20

TFORCEXPPPP $(\mathrm{I})=($ TFORCEXPP $(\mathrm{I})+$ TFORCEXPP $(\mathrm{I}+1)+\mathrm{TFORCEXPP}(\mathrm{I}+2)+\mathrm{TFO}$ RCEXPP(I+3)+TFORCEXPP (I+4)+TFORCEXPP(I+5)+TFORCEXPP(I+6)+TFORC EXPP(I+7)+TFORCEXPP(I+8)+TFORCEXPP(I+9)+TFORCEXPP(I+10)+TFORCE XPP(I+11)+TFORCEXPP(I+12)+TFORCEXPP(I+13)+TFORCEXPP(I+14)+TFORC $\operatorname{EXPP}(\mathrm{I}+15)+\mathrm{TFORCEXPP}(\mathrm{I}+16)+\mathrm{TFORCEXPP}(\mathrm{I}+17)+\mathrm{TFORCEXPP}(\mathrm{I}+18)+\mathrm{TFOR}$ $\operatorname{CEXPP}(\mathrm{I}+19)) / 20$

TREATEDTRACE $(0)=$ TREATEDTRACE $(1)$ 
TDISTANCEXPP(1)=(TREATEDTRACE $(0)+$ TREATEDTRACE $(1)+$ TREATEDTR ACE(2)+TREATEDTRACE(3)+TREATEDTRACE(4)+TREATEDTRACE(5)+TRE ATEDTRACE(6)+TREATEDTRACE(7)+TREATEDTRACE(8)+TREATEDTRAC E(9)+TREATEDTRACE(10)+TREATEDTRACE(11)+TREATEDTRACE(12)+TRE ATEDTRACE(13)+TREATEDTRACE(14)+TREATEDTRACE(15)+TREATEDTR ACE(16)+TREATEDTRACE(17)+TREATEDTRACE(18)+TREATEDTRACE(18))/ 20

TDISTANCEXPP $(\mathrm{I})=($ TREATEDTRACE $(\mathrm{I})+\mathrm{TREATEDTRACE}(\mathrm{I}+1)+\mathrm{TREATEDT}$ RACE(I+2)+TREATEDTRACE(I+3)+TREATEDTRACE(I+4)+TREATEDTRACE( $\mathrm{I}+5)+\mathrm{TREATEDTRACE}(\mathrm{I}+6)+\mathrm{TREATEDTRACE}(\mathrm{I}+7)+\mathrm{TREATEDTRACE}(\mathrm{I}+8)+\mathrm{T}$ REATEDTRACE(I+9)+TREATEDTRACE(I+10)+TREATEDTRACE(I+11)+TREA TEDTRACE(I+12)+TREATEDTRACE(I+13)+TREATEDTRACE(I+14)+TREATE DTRACE(I+15)+TREATEDTRACE(I+16)+TREATEDTRACE(I+17)+TREATEDT RACE(I+18)+TREATEDTRACE(I+19))/20

END DO

DO $\mathrm{I}=1,\left(5^{*} \mathrm{~N}\right)$

IF (I.GT.N) THEN

TFORCEXPPPP $(\mathrm{I})=$ TFORCEXPPPP $(\mathrm{N})$

TDISTANCEXPP(I)=TDISTANCEXPP(N)

END IF

IF (N.GT. $(5 * I-5))$ THEN

TFORCEXP(1)=(TFORCEXPPPP(1)+TFORCEXPPPP(2)+TFORCEXPPPP $(3)+$ TFO RCEXPPPP(4)+TFORCEXPPPP(5))/5

TFORCEXP $(\mathrm{I})=\left(\right.$ TFORCEXPPPP $\left(5^{*} \mathrm{I}\right)+\mathrm{TFORCEXPPPP}\left(5^{*} \mathrm{I}-1\right)+\mathrm{TFORCEXPPPP}(5$ *I-2)+TFORCEXPPPP(5*I-3)+TFORCEXPPPP(5*I-4))/5 
TDISTANCE(1)=(TDISTANCEXPP(1)+TDISTANCEXPP(2)+TDISTANCEXPP(3) +TDISTANCEXPP(4)+TDISTANCEXPP(5))/5

TDISTANCE $(\mathrm{I})=\left(\right.$ TDISTANCEXPP $\left(5^{*} \mathrm{I}\right)+\mathrm{TDISTANCEXPP}\left(5^{*} \mathrm{I}-1\right)+\mathrm{TDISTANCEX}$

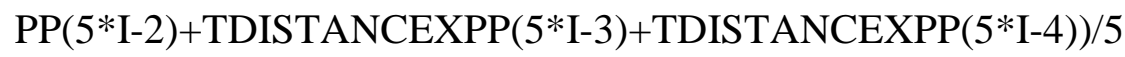

ELSE IF (N.LT.(5*I-5)) THEN

$\mathrm{TZ}=\mathrm{I}$

EXIT

END IF

END DO

MAXTFORCE $=-1$

DO $\mathrm{I}=1, \mathrm{TZ}$

IF (TFORCEXP(I).GT.MAXTFORCE) THEN

MAXTFORCE=TFORCEXP(I)

DISTANCEMAXTFORCE=TDISTANCE(I)

END IF

END DO

DO $\mathrm{I}=1, \mathrm{TZ}$

$\operatorname{TDIFF}(1)=0 \mathrm{D} 0$

TDIFF(I)=-(TFORCEXP(I)-TFORCEXP(I-1))/(TDISTANCE(I)-TDISTANCE(I-1))

MEANDIFF=-(MAXTFORCE-TFORCEXP(1))/(DISTANCEMAXTFORCE-TDIST $\operatorname{ANCE}(1))$ 


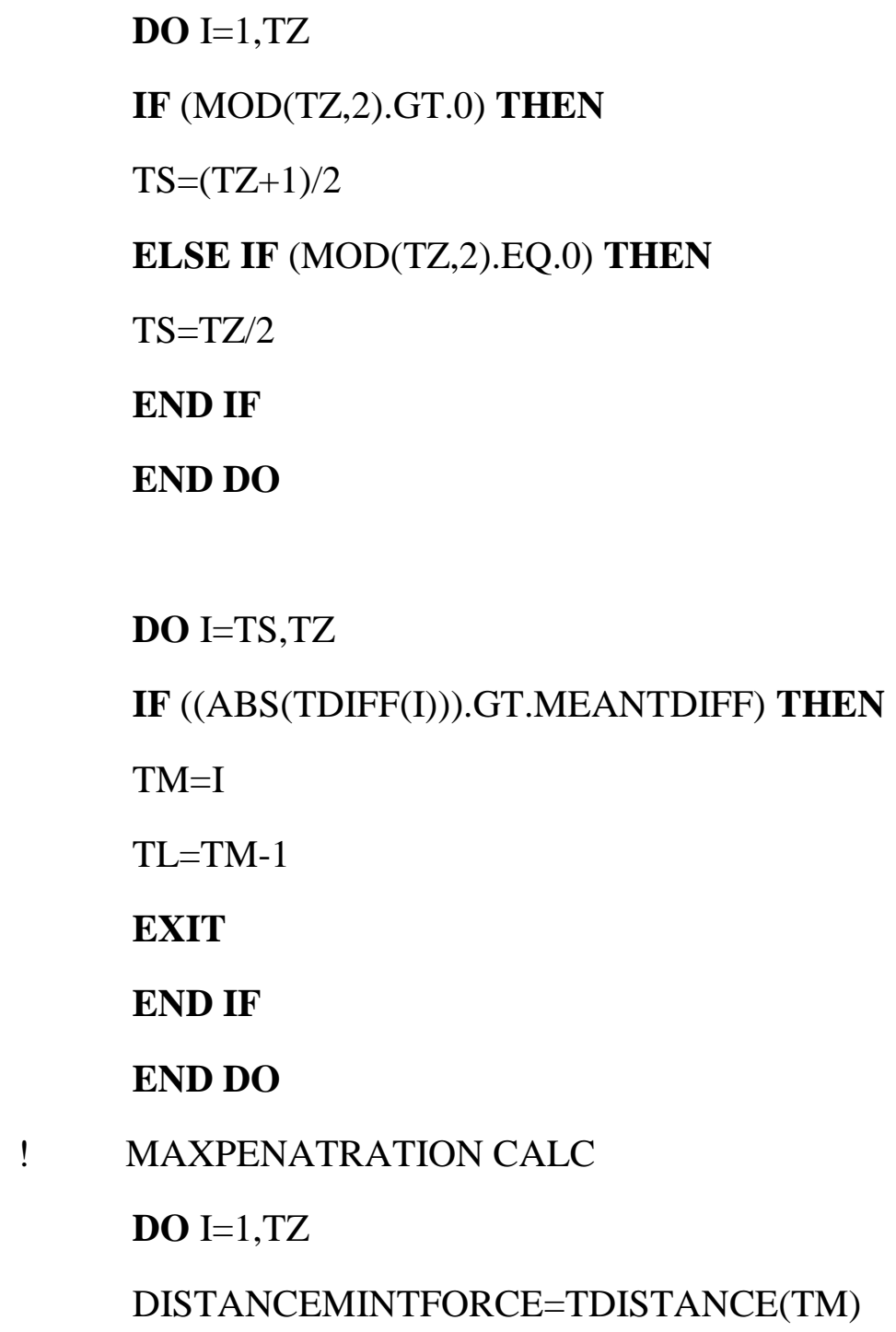


ELSE IF (TDISTANCE(I).GT.DISTANCEMINTFORCE) THEN TPENATRATION $(\mathrm{I})=0 \mathrm{D} 0$

\section{ENDIF}

END DO

DO $\mathrm{I}=1, \mathrm{TZ}$

$! \quad$ CONTACT RADIUS

$\mathrm{TA}(\mathrm{I})=\left(\mathrm{R} * * 2-(\mathrm{R}-\mathrm{TPENATRATION}(\mathrm{I}))^{* * 2}\right) * * 0.5$

AMAX $=(\mathrm{R} * * 2-(\mathrm{R}-\mathrm{TPENATRATIONMAX}) * * 2) * * 0.5$

END DO

DO $\mathrm{I}=1, \mathrm{TZ}$

! MEAN ELASTIC CONSTANT CALC

$\mathrm{TE}=3 * \mathrm{R} * \mathrm{MAXTFORCE} /(4 *(\mathrm{AMAX} * * 3))$

! HERTZ FORCE\&FLAT PUNCH\&EXTENDED FORCE CALC

IF (TPENATRATION(I).GT.0D0) THEN

$\operatorname{THERTZ}(\mathrm{I})=(4 * \mathrm{TE} * \mathrm{TA}(\mathrm{I}) * 3) /(3 * \mathrm{R})$

TFLATPUNCH(I)=TFORCEXP(I)-THERTZ(I)

EXTENDEDTFORCE $(\mathrm{I})=0 \mathrm{D} 0$

END IF

IF (TPENATRATION(I).EQ.0D0) THEN

THERTZ $(\mathrm{I})=0 \mathrm{D} 0$

TFLATPUNCH $(\mathrm{I})=0 \mathrm{D} 0$

EXTENDEDTFORCE $(\mathrm{I})=$ TFORCEXP(I)

END IF

END DO

DO $\mathrm{I}=1, \mathrm{TZ}$

! JKR CONTACT ENERGY/AREA ESTIMATE 
$\mathrm{TW} 1(\mathrm{I})=-\left(\mathrm{TFLATPUNCH}(\mathrm{I}) /\left(2 *\left(\left(2 * \mathrm{PI}^{*} \mathrm{TE} *(\mathrm{TA}(\mathrm{I}) * * 3)\right) * * 0.5\right)\right)\right) * * 2$

! DMT CONTACT ENERGY ESTIMATE

TW2(I)=EXTENDEDTFORCE $(\mathrm{I}) /(2 * \mathrm{PI} * \mathrm{R})$

END DO

!=====DEFINE JKR\&DMT ==========

DO $\mathrm{I}=1, \mathrm{TZ}$

IF (TW1(I).NE.0D0) THEN

$\mathrm{TM}=\mathrm{I}-1$

EXIT

END IF

END DO

\section{RETURN}

END SUBROUTINE

!==================END OF SUBROUTINE========================

!========SURFACE BENDING CALCULATION=============

SUBROUTINE SBCAL (SX,SY,SR)

IMPLICIT DOUBLE PRECISION (A-Z)

INTEGER $* 4$ I,N,DIM

PARAMETER $(\mathrm{DIM}=100000)$

!=====SURFACE BENDING RADIUS CALCULATION=====

$\mathrm{SW}=\mathrm{SX} / 2$

$\mathrm{SR}=((\mathrm{SW} * * 2)+(\mathrm{SY} * * 2)) /(2 * \mathrm{SY})$

RETURN

END SUBROUTINE 

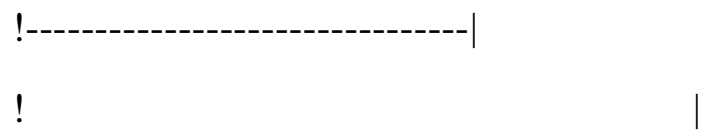

! ।

! LINE3: FORCE CURVE TREATMENT |

!

$!$

1

!==========ZETA POTENTIAL ANALYSIS $============$

SUBROUTINE ZPA

(TL,RR,IONSTR,AR,SIGMA1,TDISTANCE,TW2,ZETA)

IMPLICIT DOUBLE PRECISION (A-Z)

INTEGER*4 I,TL,J,DIM,FN,K,AR,AW,AB,S

PARAMETER $(\mathrm{DIM}=100000)$

DIMENSION

TDISTANCE(0:DIM),ZPOTENTIAL(0:DIM),ZGAPR(0:DIM),ZGAPH(0:DIM),ZG APA(0:DIM),ZSTEP(0:DIM),TW2(0:DIM),MEANZGAPH1(0:DIM),MEANZGAP H2(0:DIM),ZSTEP1(0:DIM),ZSTEP2(0:DIM)

$!=====$ CONSTACT INPUT AND UNITS SYNC=============

$! \quad$ PI

$\mathrm{PI}=\mathrm{DACOS}(-1 \mathrm{D} 0)$ 
! ACCURACY GAP

$\mathrm{AB}=\mathrm{AR}+1$

! RADIUS IN M

$\mathrm{R}=\mathrm{RR} / 2000000$

! Boltzmann constant in $\mathrm{J} / \mathrm{K}$

$\mathrm{BOLTZC}=1.38 \mathrm{D}-23$

! Avogadro constant in $1 / \mathrm{mol}$

AVOGADRO=6.0225D23

! Temperature in Kelvin $(=25 \mathrm{oC})$

TEMP=298.15D0

! Electric field constant in $\mathrm{C} / \mathrm{V} / \mathrm{m}$

ELEFIELDC $=8.854 \mathrm{D}-12$

! Elemental electron charge in $\mathrm{J} / \mathrm{V}$

CHARGE $=1.602 \mathrm{D}-19$

! Dielectric constant for water at $25 \mathrm{oC}$

DIELEC $=78.55 \mathrm{D} 0$

$\mathrm{C}=\mathrm{CC} * 1000$

!=====DEBYE LENGTH CALC $===========$

DEBYE $=\left(((\mathrm{CHARGE} * * 2) *\right.$ AVOGADRO $* 2 * \mathrm{C}) /\left(\mathrm{DIELEC}^{*} \mathrm{ELEFIELDC}^{*}\right.$ BOLTZC $^{*}$ TEMP))**-0.5

!=====FIND ZETA POTENTIAL POSITION=============

DO $\mathrm{I}=1, \mathrm{TL}$

ZPOTENTIAL $(\mathrm{I})=\mathrm{TW} 2(\mathrm{I})$

MAXZPOTENTIAL=ZPOTENTIAL(TL)-ZPOTENTIAL(TL-1)

ZMEASURE=TDISTANCE(TL-1)-TDISTANCE(TL)

TOTALZPOTENTIAL=MAXZPOTENTIAL*PI* $(\mathrm{R} * * 2)$ 


\section{END DO}

!=====AREA/HIGHT INTEGRATION PARAMETER CALC========

DO $\mathrm{K}=1, \mathrm{AB}$

! $\quad$ FIND GAP

$\mathrm{ZGAP}=\mathrm{R} / \mathrm{AR}$

! $\quad$ FIND RADIUS OF EACH STAGE OF CALC POINTS

! $\quad \operatorname{ZGAPR}(1)=0$

ZGAPR $(\mathrm{K})=$ ZGAPR $(\mathrm{K}-1)+\mathrm{ZGAP}$

! $\quad$ FIND HIGH FOR EACH STAGE

$\mathrm{ZGAPH}(\mathrm{K})=\mathrm{R}-\left((\mathrm{R} * * 2)-\left((\mathrm{ZGAPR}(\mathrm{K}))^{* * 2}\right)\right) * * 0.5$

! $\quad$ MEANZGAPH $(1)=0$

MEANZGAPH1(1)=(ZGAPH(1)-ZGAPH(0))/EXP(DEBYE/DEBYE)

MEANZGAPH1 $(\mathrm{K})=(\mathrm{ZGAPH}(\mathrm{K})-\mathrm{ZGAPH}(\mathrm{K}-1)) /(\mathrm{LOG}(\mathrm{ZGAPH}(\mathrm{K}) / \mathrm{ZGAPH}(\mathrm{K}-1)))$

MEANZGAPH2(K)=MEANZGAPH1(K)+ZMEASURE

! FIND AREA OF EACH GAP

! $\quad$ ZGAPA $(1)=0$

$\mathrm{ZGAPA}(\mathrm{K})=2 * \mathrm{PI} * \mathrm{R} *(((\mathrm{ZGAPH}(\mathrm{K})))-((\mathrm{ZGAPH}(\mathrm{K}-1))))$

!

AREARATIO $(\mathrm{K})=(2 * \mathrm{PI} * \mathrm{ZGAPR}(\mathrm{K}) * \mathrm{ZGAPH}(\mathrm{K})-2 * \mathrm{PI} * \mathrm{ZGAPR}(\mathrm{K}-1) * \mathrm{ZGAPH}(\mathrm{K}-1$ )$) / Z G A P A(K)$

$! \quad$ FIND PARAMETER OF EACH ZGAP

ZSTEP1(K)=ZGAPA(K)*EXP(-(MEANZGAPH1(K))/DEBYE)

ZSTEP2(K)=ZGAPA(K)*EXP(-(MEANZGAPH2(K))/DEBYE)

$\operatorname{ZSTEP}(\mathrm{K})=\mathrm{ZSTEP} 1(\mathrm{~K}) !-\mathrm{ZSTEP} 2(\mathrm{~K})$

! OVERALL PARAMETER

ZPARA=SUM(ZSTEP,MASK=ZSTEP.GT.0)

END DO 
!=====ZETA POTENTIAL CALC=========

ZETA=TOTALZPOTENTIAL/(ZPARA*SIGMA1)

\section{RETURN}

END SUBROUTINE

!==================END OF SUBROUTINE========================

$!============================$ SURFACE RATIO OF ZETA POTENTIAL

ANALYSIS.FOR===============================

!THE SURFACE RATIO CALCULATION OF AFM ZETA POTENTIAL

COMPUTATION

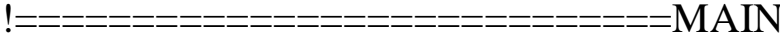

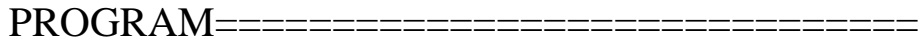

SUBROUTINE SRCALOZPA (AP,IONSTR,SR,RR,RATIO)

IMPLICIT DOUBLE PRECISION (A-Z)

INTEGER*4 K,AP,AB,DIM

PARAMETER $(\mathrm{DIM}=100000)$

\section{DIMENSION}

SGAPR(0:DIM),SDIFF(0:DIM),SGAPH(0:DIM),SGAPHH(0:DIM),MEANSGAPH1

(0:DIM),MEANSGAPH2(0:DIM),SGAPA(0:DIM),SSTEP1(0:DIM),SSTEP2(0:DIM )

!=====CONSTACT INPUT AND UNITS SYNC=============

$! \quad$ PI

$\mathrm{PI}=\mathrm{DACOS}(-1 \mathrm{D} 0)$

! ACCURACY GAP

$\mathrm{AB}=\mathrm{AP}+1$ 
! RADIUS IN M

$\mathrm{R}=\mathrm{RR} / 2000000$

$\mathrm{RS}=\mathrm{SR} / 1000000$

! Boltzmann constant in $\mathrm{J} / \mathrm{K}$

$\mathrm{BOLTZC}=1.38 \mathrm{D}-23$

! Avogadro constant in $1 / \mathrm{mol}$

AVOGADRO $=6.0225 \mathrm{D} 23$

! Temperature in Kelvin $(=25 \mathrm{oC})$

TEMP=298.15D0

! Electric field constant in $\mathrm{C} / \mathrm{V} / \mathrm{m}$

ELEFIELDC $=8.854 \mathrm{D}-12$

! Elemental electron charge in $\mathrm{J} / \mathrm{V}$

CHARGE $=1.602 \mathrm{D}-19$

! Dielectric constant for water at $250 \mathrm{CC}$

DIELEC $=78.55 \mathrm{D} 0$

! TRANSFER IONIC STRENGTH UNIT

$\mathrm{C}=\mathrm{IONSTR} * 1000$

$!=====$ DEBYE LENGTH CALC $===========$

DEBYE $=(((\mathrm{CHARGE} * * 2) *$ AVOGADRO $* 2 * \mathrm{C}) /($ DIELEC $*$ ELEFIELDC $*$ BOLTZC $*$

TEMP))**-0.5

!=====AREA/HIGHT INTEGRATION PARAMETER CALC $========$

DO $\mathrm{K}=1, \mathrm{AB}$

! $\quad$ FIND GAP

$\mathrm{SGAP}=\mathrm{R} / \mathrm{AP}$

! $\quad$ FIND RADIUS OF EACH STAGE OF CALC POINTS

$\operatorname{SGAPR}(\mathrm{K})=\operatorname{SGAPR}(\mathrm{K}-1)+\operatorname{SGAP}$

$\operatorname{SDIFF}(\mathrm{K})=\mathrm{RS}-((\mathrm{RS} * * 2)-(\operatorname{SGAPR}(\mathrm{K})) * * 2) * * 0.5$ 
! FIND MEAN HIGH FOR EACH FLAT STAGE

$\mathrm{SGAPH}(\mathrm{K})=\mathrm{R}-((\mathrm{R} * * 2)-((\mathrm{SGAPR}(\mathrm{K})) * * 2)) * * 0.5$

MEANSGAPH1(1)=(SGAPH(1)-SGAPH(0))/EXP(DEBYE/DEBYE)

MEANSGAPH1 $(\mathrm{K})=(\mathrm{SGAPH}(\mathrm{K})-\mathrm{SGAPH}(\mathrm{K}-1)) /(\mathrm{LOG}(\mathrm{SGAPH}(\mathrm{K}) / \mathrm{SGAPH}(\mathrm{K}-1)))$

! $\quad$ FIND MEAN HEIGHT FOR NON-FLAT STAGE

$\operatorname{SDIFF}(\mathrm{K})=\mathrm{RS}-\left(\left(\mathrm{RS}^{* * 2}\right)-(\operatorname{SGAPR}(\mathrm{K})) * * 2\right) * * 0.5$

$\operatorname{SGAPHH}(\mathrm{K})=\mathrm{SGAPH}(\mathrm{K})-\operatorname{SDIFF}(\mathrm{K})$

MEANSGAPH2(1)=(SGAPHH(1)-SGAPHH(0))/EXP(DEBYE/DEBYE)

MEANSGAPH2(K)=(SGAPHH(K)-SGAPHH(K-1))/(LOG(SGAPHH(K)/SGAPHH ( $\mathrm{K}-1)))$

! FIND AREA OF EACH GAP

$\operatorname{SGAPA}(\mathrm{K})=\mathrm{PI} *(((\operatorname{SGAPR}(\mathrm{K})) * * 2)-((\operatorname{SGAPR}(\mathrm{K}-1)) * * 2))$

! $\quad$ FIND PARAMETER OF EACH GAP (FLAT)

SSTEP1(K)=SGAPA(K)*EXP(-(MEANSGAPH1(K))/DEBYE)

! FIND PARAMETER OF EACH GAP (NON-FLAT)

$\operatorname{SSTEP} 2(\mathrm{~K})=\operatorname{SGAPA}(\mathrm{K}) * \operatorname{EXP}(-(\mathrm{MEANSGAPH} 2(\mathrm{~K})) / \mathrm{DEBYE})$

! OVERALL PARAMETER RATIO

PARA1 $=$ SUM(SSTEP1,MASK=SSTEP1.GT.0) $\quad ! \quad$ FLAT

PARA2 $=$ SUM(SSTEP2,MASK=SSTEP2.GT.0) ！ NON-FLAT

RATIO=PARA2/PARA1

END DO

RETURN

END SUBROUTINE

!==================END OF SUBROUTINE======================== 
!============================AFM RETRACE ADHESION

ANALYSIS.FOR===============================

!ADHESION ANALYSIS

!JKR AND DMT MODEL APPLIED

!============================MAIN

PROGRAM==============================

SUBROUTINE AFMRTAA

(K,B,RR,TREATEDRETRACE,TREATEDRETRACEFORCE,POINT2,RPENATR ATIONMAX,RE,TURN1,RY,RZ,DEFINE,RDISTANCE,RW2,RFORCEXP,MINRF ORCE)

IMPLICIT DOUBLE PRECISION (A-Z)

INTEGER*4

I,B,J,DIM,FN,RM,RL,RZ,RS,RY,RX,SS,DEFINE,RRS,RRX,RRL,REALX,TURN,S B,K,LINE,TURN1

PARAMETER $(\mathrm{DIM}=100000)$

DIMENSION

TREATEDRETRACEFORCE(0:DIM),RFORCER(0:DIM),RFORCEXP(0:DIM),RF ORCEXPP(0:DIM),RDISTANCE(0:DIM),RA(0:DIM),RPENATRATION(0:DIM),R HERTZ(0:DIM),RFLATPUNCH(0:DIM),RW1(0:DIM),EXTENDEDRFORCE(0:DI M),RW2(0:DIM),RDIFF(0:DIM),TREATEDRETRACE(0:DIM),RFORCEXPPPP(0: DIM),RDISTANCEXPP(0:DIM),RFORCEX(0:DIM),RDISTANCEX(0:DIM),POIN T(0:DIM),POINT1(0:DIM),POINT2(0:DIM),RDIFF1(0:DIM),DEFINE1(0:DIM),AA VE(0:DIM),VV(0:DIM),COLLAPSEHIGHT(0:DIM),COLLAPSEAREA(0:DIM),E MODULUS(0:DIM),AMAX(0:DIM),DISTMAX(0:DIM),AMIN(0:DIM),DISTMIN( 0:DIM),PULLHIGHT(0:DIM)

$!=====$ CONSTACT INPUT AND UNITS SYNC============= $! \quad$ PI 


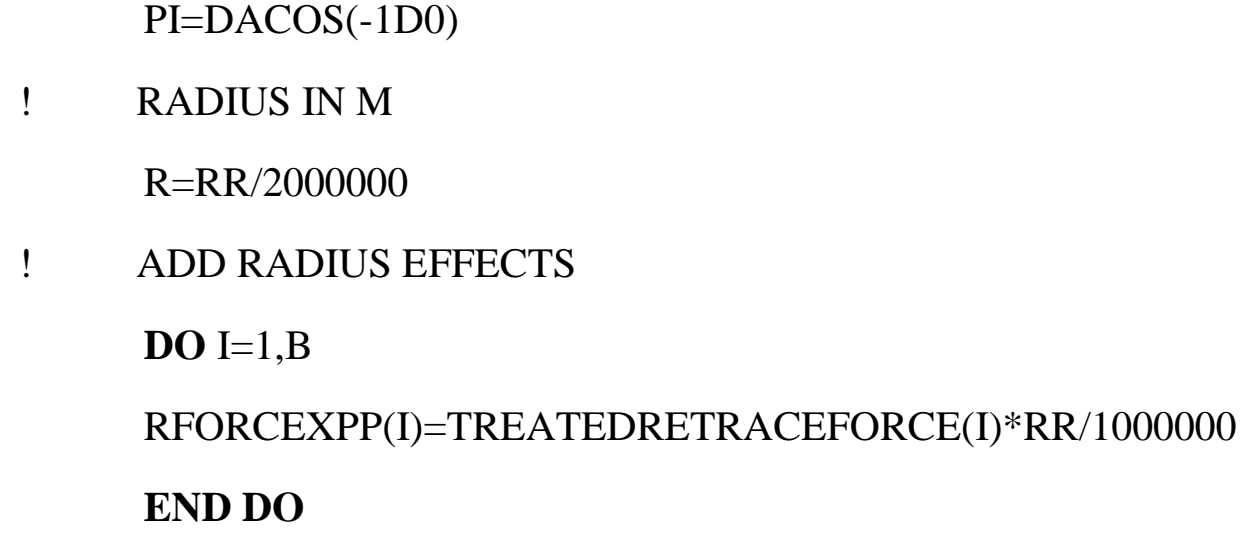


ORCEXPP(I+15)+RFORCEXPP(I+16)+RFORCEXPP(I+17)+RFORCEXPP(I+18)+ RFORCEXPP(I+19))/20

TREATEDRETRACE(0)=TREATEDRETRACE $(1)$

RDISTANCEXPP $(1)=($ TREATEDRETRACE $(0)+$ TREATEDRETRACE $(1)+$ TREAT EDRETRACE(2)+TREATEDRETRACE(3)+TREATEDRETRACE(4)+TREATEDR ETRACE(5)+TREATEDRETRACE(6)+TREATEDRETRACE(7)+TREATEDRETR ACE(8)+TREATEDRETRACE(9)+TREATEDRETRACE(10)+TREATEDRETRAC E(11)+TREATEDRETRACE(12)+TREATEDRETRACE(13)+TREATEDRETRAC E(14)+TREATEDRETRACE(15)+TREATEDRETRACE(16)+TREATEDRETRAC E(17)+TREATEDRETRACE(18)+TREATEDRETRACE(18))/20

RDISTANCEXPP $(\mathrm{I})=($ TREATEDRETRACE $(\mathrm{I})+\mathrm{TREATEDRETRACE}(\mathrm{I}+1)+\mathrm{TREA}$ TEDRETRACE(I+2)+TREATEDRETRACE(I+3)+TREATEDRETRACE(I+4)+TRE ATEDRETRACE(I+5)+TREATEDRETRACE(I+6)+TREATEDRETRACE(I+7)+TR EATEDRETRACE(I+8)+TREATEDRETRACE(I+9)+TREATEDRETRACE(I+10)+ TREATEDRETRACE(I+11)+TREATEDRETRACE(I+12)+TREATEDRETRACE(I +13)+TREATEDRETRACE(I+14)+TREATEDRETRACE(I+15)+TREATEDRETR ACE(I+16)+TREATEDRETRACE(I+17)+TREATEDRETRACE(I+18)+TREATED RETRACE(I+19))/20

END DO

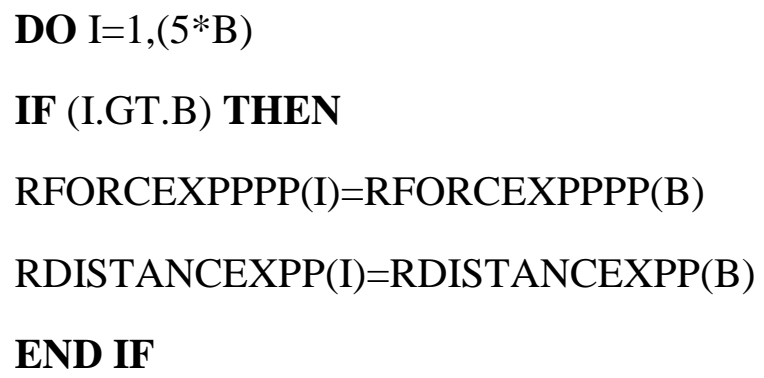


RFORCEX(1)=(RFORCEXPPPP(1)+RFORCEXPPPP(2)+RFORCEXPPPP(3)+RFO RCEXPPPP(4)+RFORCEXPPPP(5))/5

$\operatorname{RFORCEX}(\mathrm{I})=\left(\operatorname{RFORCEXPPPP}\left(5^{*} \mathrm{I}\right)+\mathrm{RFORCEXPPPP}\left(5^{*} \mathrm{I}-1\right)+\mathrm{RFORCEXPPPP}\left(5^{*}\right.\right.$ I-2)+RFORCEXPPPP(5*I-3)+RFORCEXPPPP $(5 * I-4)) / 5$

RDISTANCEX $(1)=($ RDISTANCEXPP $(1)+$ RDISTANCEXPP $(2)+$ RDISTANCEXPP ( 3)+RDISTANCEXPP(4)+RDISTANCEXPP(5))/5

RDISTANCEX $(\mathrm{I})=\left(\operatorname{RDISTANCEXPP}\left(5^{*} \mathrm{I}\right)+\mathrm{RDISTANCEXPP}\left(5^{*} \mathrm{I}-1\right)+\mathrm{RDISTANC}\right.$ $\left.\operatorname{EXPP}\left(5^{*} \mathrm{I}-2\right)+\mathrm{RDISTANCEXPP}\left(5^{*} \mathrm{I}-3\right)+\mathrm{RDISTANCEXPP}\left(5^{*} \mathrm{I}-4\right)\right) / 5$

\section{END IF}

IF (B.LT. $(5 * \mathrm{I}+5))$ THEN

$\mathrm{RZ}=\mathrm{I}$

EXIT

END IF

END DO

DO $\mathrm{I}=1, \mathrm{RZ}$

RFORCEXP(I)=RFORCEX $(\mathrm{I})$

RDISTANCE $(\mathrm{I})=$ RDISTANCEX $(\mathrm{I})$

END DO

DO I=1,RZ

RFORCER(I)=RFORCEXP(RZ+1-I $)$

END DO

$!=====$ FIND MAXIMUM AND MINIMUM FORCE $======================$ 
MAXRFORCE $=-1$

DO I=1,RZ

IF (RFORCEXP(I).GT.MAXRFORCE) THEN

MAXRFORCE=RFORCEXP(I)

DISTANCEMAXRFORCE=RDISTANCE(I)

$\mathrm{RY}=\mathrm{I}$

END IF

END DO

MINRFORCE $=1$

DO $\mathrm{I}=1, \mathrm{RZ}$

IF (RFORCEXP(I).LT.MINRFORCE) THEN

MINRFORCE=RFORCEXP(I)

DISTANCEMINRFORCE=RDISTANCE(I)

$\mathrm{RX}=\mathrm{I}$

END IF

END DO

!=====FIND SLOPE AND DEFINE SURFACE

SEPARATION======================

DO I=RY,RZ

$\mathrm{RDIFF}(\mathrm{I})=\mathrm{ABS}(\mathrm{RFORCEXP}(\mathrm{I}+1)-\mathrm{RFORCEXP}(\mathrm{I})) / \mathrm{ABS}(\mathrm{RDISTANCE}(\mathrm{I}+1)-\mathrm{RDIST}$ $\operatorname{ANCE}(\mathrm{I}))$

MEANRDIFF=-(MAXRFORCE-MINRFORCE)/(DISTANCEMAXRFORCE-DIST ANCEMINRFORCE) 


\section{END DO}

DO I=RY,RZ

IF ((RDIFF(I)).LT.MEANRDIFF) THEN

$\mathrm{RM}=\mathrm{I}$

$\mathrm{RL}=\mathrm{RM}+1$

EXIT

END IF

END DO

!=====ADHESION DETERMINATION: POINT OF ADHESION

SELECTION $======================$

DO I=RX,RZ

IF ((MINRFORCE+0.05*MAXRFORCE).GT.0D0) THEN

IF (MINRFORCE.NE.(RFORCEXP(RZ))) THEN

$\mathrm{BREAK}=1$

IF (BREAK.GT.RFORCEXP(I)) THEN

BREAK $=$ RFORCEXP(I)

RDISTANCEBREAK=RDISTANCE(I)

$\mathrm{SDS}=\mathrm{I}$

END IF

IF (DISTANCEMINRFORCE.GE.RDISTANCEBREAK) THEN

MAXADHESION $=0$

$\mathrm{RS}=0$

DEFINE $=0$

ELSE IF (DISTANCEMINRFORCE.LT.RDISTANCEBREAK) THEN

MAXADHESION=BREAK-MINRFORCE

DEFINE $=2$ 
$\mathrm{RS}=0$

END IF

EXIT

ELSE IF (MINRFORCE.EQ.(RFORCEXP(RZ))) THEN

DEFINE $=0$

END IF

ELSE IF ((MINRFORCE+0.05*MAXRFORCE).LE.0D0) THEN

$\mathrm{BREAK}=-1$

IF (BREAK.LT.RFORCEXP(I)) THEN

BREAK $=$ RFORCEXP(I)

$\mathrm{SDS}=\mathrm{I}$

END IF

MAXADHESION=BREAK-MINRFORCE

DEFINE $=1$

END IF

END DO

IF (DEFINE.EQ.1) THEN

DO I=1,RZ

IF (((RFORCER(I))+0.05*MAXRFORCE).LE.(0D0)) THEN

$\mathrm{SS}=\mathrm{I}$

$\mathrm{RS}=\mathrm{RZ}+1-\mathrm{I}$

EXIT

END IF

END DO

END IF

!REAL POINT OF ADHESION STOP

$\mathrm{RRS}=5 * \mathrm{RS}$ 
!REAL POINT OF MAX ADHESION

$\mathrm{RRX}=5 * \mathrm{RX}$

!REAL ADHESION START POINT

$\mathrm{RRL}=5 * \mathrm{RL}$

!=====ADHESION TYPE ANALYSIS FOR SELECTED

PART $======================$

IF (DEFINE.EQ.0) THEN

REALADHESION=0D0

TURN $=0$

$\mathrm{LINE}=0$

TURN1 $=0$

ELSE IF (DEFINE.EQ.1) THEN

DO I=(RRX-20), $(\mathrm{RRX}+20)$

REALADHESION $=1$

IF (REALADHESION.LT.TREATEDRETRACEFORCE(I)) THEN

REALADHESION=TREATEDRETRACEFORCE(I)

REALX=I

END IF

END DO

DO I=1,DIM

$\operatorname{RDIFF} 1(\mathrm{I})=0$

END DO

!ESTIMATE TYPE OF ADHESION (DEVIDE ADHESION CURVE INTO PARTS) 
TURN=0

DO $\mathrm{I}=\mathrm{RX}, \mathrm{RS}$

RDIFF1 $(\mathrm{I})=((\mathrm{RFORCEXP}(\mathrm{I}+1)-\mathrm{RFORCEXP}(\mathrm{I})) /(\mathrm{RDISTANCE}(\mathrm{I}+1)-\mathrm{RDISTANCE}(\mathrm{I}$ )$)+(1 \mathrm{D}-10)$

IF ((RDIFF1(I-1)*RDIFF1(I)).LT.0D0) THEN

IF ((ABS(RDIFF1(I-1)*RDIFF1(I))).GT.(1D-15)) THEN

TURN=TURN+1

$\mathrm{SB}=\mathrm{I}$

DO $\mathrm{K}=1, \mathrm{TURN}$

POINT1 $($ TURN $)=S B$

END DO

END IF

END IF

END DO

DO $\mathrm{K}=1$,TURN

$\operatorname{POINT}(\mathrm{K})=5 * \operatorname{POINT} 1(\mathrm{~K})$

END DO

LINE=TURN +1

TURN1=LINE+1

! CALCULATE THE MAX AND MIN ADHESION

DO I=1,TURN1

POINT2 $(\mathrm{I}+1)=$ POINT1 $(\mathrm{I})$

POINT2(1) $=\mathrm{RX}$

POINT2(TURN1)=RS

END DO 
DO $\mathrm{K}=1, \mathrm{TURN} 1$

DO I=POINT2(K),POINT2(K+1)

$\operatorname{AMAX}(\mathrm{K})=-1$

IF (AMAX(K).LT.RFORCEXP(I)) THEN

$\operatorname{AMAX}(\mathrm{K})=\mathrm{RFORCEXP}(\mathrm{I})$

DISTMAX $(\mathrm{K})=$ RDISTANCE $(\mathrm{I})$

END IF

$\operatorname{AMIN}(K)=1$

IF (AMIN(K).GT.RFORCEXP(I)) THEN

$\operatorname{AMIN}(\mathrm{K})=\mathrm{RFORCEXP}(\mathrm{I})$

$\operatorname{DISTMIN}(\mathrm{K})=\mathrm{RDISTANCE}(\mathrm{I})$

END IF

$\operatorname{AAVE}(\mathrm{K})=\mathrm{SUM}(\mathrm{RFORCEXP}) /(\mathrm{POINT} 2(\mathrm{~K}+1)+1-\mathrm{POINT} 2(\mathrm{~K}))$

!DEFINE PULL OUT, STABLIZE AND ADHESIONC COLLAPSION IF (DISTMAX(K).LT.DISTMIN(K)) THEN

$\operatorname{DEFINE} 1(\mathrm{~K})=-1 \quad$ !COLLAPSED

ELSE IF(DISTMAX(K).EQ.DISTMIN(K)) THEN

$\operatorname{DEFINE} 1(\mathrm{~K})=0 \quad$ !STABLIZED

ELSE IF (DISTMAX(K).GT.DISTMIN(K)) THEN

$\operatorname{DEFINE} 1(\mathrm{~K})=1 \quad$ !PULL OUT

END IF

!CALCULATION OF EACH CASE

IF (DEFINE1(K).EQ.0) THEN

$\mathrm{VV}(\mathrm{K})=\mathrm{AAVE}(\mathrm{K}) /(6 * \mathrm{PI} * \mathrm{R}) \quad$ !STABLIZED SITUATION:

CALCULATION OF VISCOSITY*VELOCITY

ELSE IF (DEFINE1(K).LT.0) THEN 
$\operatorname{COLLAPSEAREA}(\mathrm{K})=2 * \mathrm{PI} * \mathrm{R} * \operatorname{COLLAPSEHIGHT}(\mathrm{K})$

ELSE IF ((DEFINE1(K)).GT.0) THEN

PULLHIGHT(K)=RDISTANCE(POINT2(K+1))-RDISTANCE(POINT2(K))

$\operatorname{EMODULUS}(\mathrm{K})=(\operatorname{AMAX}(\mathrm{K})-\operatorname{AMIN}(\mathrm{K})) / \mathrm{PULLHIGHT}(\mathrm{K})$

END IF

END DO

END DO

END IF

!=====DMT\&JKR MODEL APPLICATION ON THE FORCE

STUDY======================

! MAXPENATRATION CALC

DO I=RY,RZ

DISTANCEMINRFORCE=RDISTANCE(RM)

RPENATRATIONMAX=DISTANCEMINRFORCE-DISTANCEMAXRFORCE END DO

$! \quad$ PENATRATION CALC

DO I=RY,RZ

IF (RDISTANCE(I).LT.DISTANCEMINRFORCE) THEN

RPENATRATION $(\mathrm{I})=-$ RDISTANCE(I)+DISTANCEMAXRFORCE+RPENATRAT IONMAX

ELSE IF (RDISTANCE(I).GT.DISTANCEMINRFORCE) THEN $\operatorname{RPENATRATION}(\mathrm{I})=0 \mathrm{D} 0$

ENDIF 


\section{END DO}

DO I=RY,RZ

$! \quad$ CONTACT RADIUS

$\mathrm{RA}(\mathrm{I})=\left(\mathrm{R} * * 2-(\mathrm{R}-\mathrm{RPENATRATION}(\mathrm{I}))^{* * 2}\right) * * 0.5$

RAAMAX $=(\mathrm{R} * * 2-(\mathrm{R}-\mathrm{RPENATRATIONMAX}) * * 2) * * 0.5$

\section{END DO}

DO I=RY,RZ

$! \quad$ MEAN ELASTIC CONSTANT CALC

$\mathrm{RE}=3 * \mathrm{R} * \mathrm{MAXRFORCE} /(4 *(\mathrm{RAAMAX} * * 3))$

! HERTZ FORCE\&FLAT PUNCH\&EXTENDED FORCE CALC

IF (RPENATRATION(I).GT.0D0) THEN

$\operatorname{RHERTZ}(\mathrm{I})=(4 * \mathrm{RE} * \mathrm{RA}(\mathrm{I}) * * 3) /(3 * \mathrm{R})$

RFLATPUNCH(I)=RFORCEXP(I)-RHERTZ(I)

EXTENDEDRFORCE $(\mathrm{I})=0 \mathrm{D} 0$

ELSE IF (RPENATRATION(I).EQ.0D0) THEN

RHERTZ $(\mathrm{I})=0 \mathrm{D} 0$

RFLATPUNCH $(\mathrm{I})=0 \mathrm{D} 0$

EXTENDEDRFORCE $(\mathrm{I})=$ RFORCEXP(I)

END IF

END DO

DO I=RY,RZ

! JKR CONTACT ENERGY/AREA ESTIMATE

$\mathrm{RW} 1(\mathrm{I})=-\left(\mathrm{RFLATPUNCH}(\mathrm{I}) /\left(2 *\left(\left(2 * \mathrm{PI}^{*} \mathrm{RE} *(\mathrm{RA}(\mathrm{I}) * * 3)\right) * * 0.5\right)\right)\right) * * 2$

$! \quad$ DMT CONTACT ENERGY ESTIMATE 
RW2(I)=EXTENDEDRFORCE $(\mathrm{I}) /(2 * \mathrm{PI} * \mathrm{R})$

END DO

RETURN

END SUBROUTINE

$!==================$ END OF MAIN

SUBROUTINE=========================" 